\title{
RHEO-NMR INVESTIGATIONS OF FLOW AND ALIGNMENT IN COMPLEX FLUIDS
}

by

Bradley Douglass

\author{
A thesis \\ submitted to the Victoria University of Wellington \\ in fullfilment of the requirements for the degree of \\ Doctor of Philosophy \\ in Physics
}

Victoria University of Wellington

The MacDiarmid Institute for Advanced Materials and

Nanotechnology

2011 


\begin{abstract}
This thesis reports the use of Rheo-NMR, that is, a class of techniques within the realm of magnetic resonance which are both confirmatory and complementary to rheometric experiments on materials which can best be classified as complex fluids. The physical properties of such fluids are both hybrid and, in general, vaguely defined. In displaying characteristics attributable to both ideal fluids and elastic solids, the term 'complex fluid', in a very real sense, epitomises all the fluids with which every human deals with (and is comprised of) daily.

With a multitude of potential candidates for further research then, here we confine ourselves to fluids of molecules and aggregates which are either linear polymers or at least maintain the curvilinear one-dimensional topology of linear polymers. Magnetic resonance is an ideal research tool in this regard, as it is in many respects a rather statistical and insensitive tool from a signal-to-samplevolume perspective, precisely the regime in which the dynamics of a macroscopic collection of macromolecules is relevant.

Material deformation is the mechanism upon which rheological measurement depends, and the first research presented here reports on a numerical simulation of the NMR signal of sheared polymer melts. Proton NMR relaxation times of such melts have previously been measured experimentally and found to depend on the shear rate applied by a horizontal Couette geometry, presumably due to the alignment of the mean-field boundaries of the space in which the polymer may reside, known as the polymer tube. The restrictions forming the tube are the other polymers in the bulk, around which an exemplar polymer molecule must meander. In diffusing through this tube, whose direction between entanglements is random in equilibrium, at any time, the return-to-origin correlation for a single spin returning to its locally anisotropic environment generates the least NMR transverse relaxation, as the sum contribution from all tube segments is random. When a deformation-related transformation matrix is applied to the coordinates of entanglements in the polymer, tube segments are no longer isotropically distributed, and an enhanced relaxation process results. Here we present the results of a numerical simulation of this procedure, based on the
\end{abstract}


earlier model of Ball, Callaghan and Samulski, in addition to measurements of the transverse NMR relaxation by Cormier. Not only does it demonstrate qualitative agreement, the NMR signal can be simulated quantitavely or conversely, the size of several key polymer physics parameters can be found through fitting to the NMR signal.

Proton NMR spectroscopy is inherently simpler than deuteron NMR spectroscopy, in which the nucleus of interest is quadrupolar. However, a large section of this thesis deals with the structures and response of worm-like micellar structures in solution, for which alignment data cannot reasonably be measured with the proton alone. The most used sample in this thesis is that of the BASF nonionic block copolymer Pluronic P105 in aqueous solution $(5 \% \mathrm{w} / \mathrm{w})$, and a small amount of 1-phenylethanol is required to stabilise cylindrical micellar structures. 1-phenylethanol is a small molecule perfectly suited to act also as a deuterated probe molecule to observe alignment, as it resides in the core of the micelle. By using a variety of Rheo-NMR techniques, such as velocimetry, spatially resolved spectroscopy, and diffusometry, many different flow and alignment behaviours were observed for this solution in Couette flow. Following the measured temperature-dependent viscosity of the P105 solution, which shows an elevated viscosity in a temperature region $\sim 15 \mathrm{~K}$ wide centred at $\sim 297 \mathrm{~K}$, we use temperature and applied shear rate as independent variables in our experiments, first identifying spectral features through diffusometry, and then observing a range of behaviours including shear-banding and quadrupolar splitting indicating alignment.

Finally we present some experimental work performed in the extensional flow geometry known as the semi-hyperbolic converging die. Extensional flow, inherently, is a transient and finite procedure, and such a geometry is designed to produce a constant extension rate along the axis of its constricting pipe, which, compared to the mill geometries, improves the volume and time over which extension occurs. We investigate the flow and alignment measuring capabilities of Rheo-NMR in this geometry. 


\section{Acknowledgment}

We live in a culture which gives the chance to study for the joy of it in a university in a great city in a great country, and that is a great thing. Within that setting, Paul has built a place that people from here and abroad love to join, visit and enjoy, and when I asked him if I could be one of those people, he took me on, which was a great thing for me. He's an incredible person who, despite all his own tribulations, somehow keeps all the pots boiling, managing seemingly endless numbers of people and projects, but more importantly in his students' cases, managing them in totally different ways depending on what it is they need to get out of their time as students to improve. I don't quite know (yet) how I can repay his consideration, guidance and tolerance, but (a) I'm glad he's around and I get a chance to, and (b) I'm the better to do it, having found out enough about myself by this opportunity, having finished this (large) chapter of life.

Having taken longer than your average PhD student to compile this thesis, I have had the opportunity to meet and be influenced by more than the average number of great people. I've seen an incredible number of people come through the lab, and I'd like to acknowledge every single one of their contributions to building a great environment to work in, but I can't list them all here. There are a few, though, that were (and are) particularly special to me academically and socially. Ryan, Sasha, Daniel, Petrik, Mark, Antoine, Kirk and Allan. The visitors, wise heads, master craftsmen and other people who have commanded my respect such as Lou, Ralph, Alan, Dave, Manu and Margaret are so vibrant and diversely skilled that they have been such an inspirational part of being around here.

But there's a whole lot to life outside the lab, and I've lived in some great places, had a million hobbies come and go, played sports with a bunch of teams, 
and every one of the people that were there for the ride I am grateful to for keeping me sane. AJ, Mike, Ryan, Piotr, Carin and Charlotte, Felicity and Jude, have all in some way done something very special for me. I'm pretty stoked to have a couple of great friends in Jon and Andrew for whom it doesn't matter how much time goes by between drinks, have always just been able to pick up a conversation where it left off.

In terms of people who are always there, though, Mum and Dad have endured quite a lot in the time it's taken me to get this done, were always around and supportive when 'enduring' wasn't yet required, and deserve special mention for even getting me to a point where any of this was even possible. Nanna was always there, too, but I didn't get this done in time for her.

To sum this all up, there are so many people that have made me the person to have finished this thesis, and in cherry-picking names I do a great injustice to everyone not explicitly listed here. People 'round here are awesome, and I acknowledge you. 


\section{Contents}

$\begin{array}{llr}1 & \text { Introduction } & 9\end{array}$

1.1 Complex Fluids . . . . . . . . . . . . . . . . . . . . . . . . 9 9

1.2 Magnetic Resonance in Materials . . . . . . . . . . . . . . . . . 10

1.3 Introduction to this Thesis . . . . . . . . . . . . . . 11

2 The Mechanics of Soft Materials and Polymers 15

2.1 What are soft materials? . . . . . . . . . . . . . . 15

2.2 Overview . . . . . . . . . . . . . . . . . 16

2.3 Deformation of continuous media : stress and strain; viscosity and elasticity . . . . . . . . . . . . . . . . 17

2.3.1 Deformation and velocity gradient tensors . . . . . . 17

2.3 .2 Stress . . . . . . . . . . . . . . . . . . 19

2.3.3 Shear viscosity . . . . . . . . . . . . . . . . 20

2.3.4 Linear viscoelasticity . . . . . . . . . . . . . . . 22

2.3.5 Simple examples of non-linear rheology in materials . . . 23

2.3.6 Dimensionless parameters . . . . . . . . . . . 24

2.3.7 Typical shearing geometries . . . . . . . . . . . . 26

2.3.8 Extensional deformation . . . . . . . . . . . . . . . 28

2.3.9 Extensional viscosity . . . . . . . . . . . . . . . 30

2.3.10 Conventional extensional rheometry . . . . . . . . . . 33

2.4 Polymer Dynamics . . . . . . . . . . . . . . . . . . . 36

2.4.1 End-to-end vector distribution . . . . . . . . . . . . 36

2.4.2 Polymer melts and the tube model . . . . . . . . . . 38

2.4.3 Reptation and the tube model . . . . . . . . . . . . 38

2.4.4 Refinements to the Doi-Edwards model of polymer dynamics 41 
2.5 Surfactants and Worm-like Micelles . . . . . . . . . . . . . . . . 41

2.5.1 Molecular aggregation . . . . . . . . . . . 41

2.5.2 Nonionic worm-like structure formation . . . . . . . . . . 43

2.5.3 Shear banding in worm-like micelles . . . . . . . . . . . 47

3 Magnetic Resonance Spectroscopy, Imaging and Velocimetry 55

3.1 Nuclear Spin . . . . . . . . . . . . . . . . . . . . . . 56

3.2 Spin and the Density Matrix . . . . . . . . . . . . 57

3.3 Spins in Magnetic Fields . . . . . . . . . . . . . . . 58

3.4 Relaxation . . . . . . . . . . . . . . . . 59

3.4.1 Phenomenological Relaxation . . . . . . . . . 59

3.4.2 Dependence of transverse relaxation on dynamics . . . . 60

3.5 The nuclear spin Hamiltonian . . . . . . . . . . . . . . . . . . . 62

3.5.1 The dipole interaction . . . . . . . . . . . 63

3.5.2 The quadrupole interaction and the spin-1 deuteron . . . 64

3.5.3 The chemical shift interaction . . . . . . . . . 66

3.6 Signal detection . . . . . . . . . . . . . . . . . . . . 67

3.7 Signal averaging and phase cycling . . . . . . . . . . 68

3.8 Magnetic Resonance Imaging . . . . . . . . . . . . . . . 70

3.8.1 The Hahn spin echo . . . . . . . . . . . . 71

3.8.2 Volume selection with field gradients . . . . . . . . 71

3.8.3 The projection profile . . . . . . . . . . . 73

3.8.4 Radial spectroscopic imaging . . . . . . . . . . . 74

3.8.5 Diffusometry . . . . . . . . . . . . . 76

3.8.6 Magnetic Resonance Velocimetry . . . . . . . . . . . 77

3.8.7 The standard pulsed-gradient spin-echo velocimetry experiment . . . . . . . . . . . . . . . 78

3.8.8 Fast imaging of velocimetry with RARE . . . . . . . 80

3.9 The Relevance of Magnetic Resonance Techniques in Rheology • 81

4 Transverse Relaxation in Sheared Polymer Melts 85

4.1 Introduction . . . . . . . . . . . . . . . . . . . . . . 85

4.1.1 Motivation . . . . . . . . . . . . 86

4.1.2 Approach and Method ............ 86 


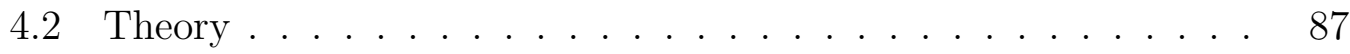

4.2.1 Dipolar relaxation function for reptating polymer segments in zero shear . . . . . . . . . . . . . . . 88

4.2.2 Dipolar relaxation function for reptating polymer segments in the presence of shear . . . . . . . . . . . 96

4.2.3 Predictions for magnetic field along the velocity and velocity gradient directions . . . . . . . . . . . . . . . 98

4.3 Experimental . . . . . . . . . . . . . . . . . 100

4.3.1 Horizontal Couette Measurement of $T_{2}$ in Polymer Melts 100

4.3.2 Numerical Computation of $T_{2}$ in Polymer Melts . . . . . 104

4.4 Results and Discussion . . . . . . . . . . . . . . . . . . 105

4.4.1 Experimental measurement of $T_{2}$ in polymer melts, and fitting model parameters . . . . . . . . . . . . . 105

4.4.2 Differences between the velocity and velocity gradient scenarios .................... . 108

4.4 .3 Discussion . . . . . . . . . . . . . . . . . . 110

5 Complex Fluids in Shear Flow 115

5.1 Overview . . . . . . . . . . . . . . . . . . 115

5.2 Apparatus ...................... 116

5.2 .1 The Rheometer . . . . . . . . . . . . . . 116

5.2 .2 Rheo-NMR .................. . . 117

5.3 Worm-like micelles . . . . . . . . . . . . . . . . . . . 118

5.4 Rheology of worm-like micelles . . . . . . . . . . . . . . . . . . . 119

5.5 Pluronic P105 . . . . . . . . . . . . . . . . . . 120

5.5.1 Sample details . . . . . . . . . . . . . . . . . . . 120

5.5.2 Rheometry.................... . . 121

5.5.3 ${ }^{1} \mathrm{H}$ spectroscopy and diffusometry . . . . . . . . . . 122

5.5.4 Deuteron NMR spectroscopy . . . . . . . . . . . . . . . . 125

5.5.5 Spatially resolved velocimetry . . . . . . . . . . . . 127

5.5.6 Spatially resolved spectroscopy . . . . . . . . . . . . 129

5.5.7 Possible models for phase behaviour . . . . . . . . . . . . 132

5.6 Conclusions . . . . . . . . . . . . . . . . . . . 137 
6 Extensional flow investigations with magnetic resonance

6.1 Introduction . . . . . . . . . . . . . . . . . . . . 143

6.2 The semi-hyperbolic converging die . . . . . . . . . . . 144

6.2.1 Design of a converging die . . . . . . . . . . . 145

6.2.2 Realisation of a converging die for magnetic resonance . 147

6.3 Basic flow issues through the glass SHCD . . . . . . . . . . 150

6.3 .1 Limitations . . . . . . . . . . . . . . . 150

6.4 SHCD contraction flows . . . . . . . . . . . . 156

6.4.1 Expectations based on prior investigations . . . . . . 156

6.4.2 Experimental velocimetry of flow through an SHCD . . . 157

6.4.3 Magnetic Resonance Spectroscopy in an SHCD . . . . . 164

6.4.4 Radial spectroscopic imaging of P105 in an SHCD . . . . 166

6.4.5 Further analysis of the spatially resolved spectra . . . . 167

6.5 Conclusions . . . . . . . . . . . . . . . . . . . 169

7 Conclusions $\quad 175$

7.1 Review of aims . . . . . . . . . . . . . . . 175

7.2 Alignment's effect on proton spectroscopy of polymer melts . . . 176

7.3 Rheo-NMR observation of the effect of shear on worm-like micelles 178

7.4 Extensional flow as measured with magnetic resonance . . . . . 180 


\section{Chapter 1}

\section{Introduction}

\subsection{Complex Fluids}

This thesis concerns itself with the investigation of the properties of complex fluids, those which display non-Newtonian, visco-elastic behaviours under flow conditions. Rheology is the branch of science dealing with the complex mechanical properties of such materials; in particular when they are taken together with microscopic properties and molecular modelling of the origins of such behaviour, the field of soft condensed matter physics is formed.

While not all complex fluids are of a polymeric nature, they are of primary interest to us here. The composition of those materials range from dilute solutions - relatively isolated macromolecules in solvents such as water, through to entangled and cross-linked molecules that are almost solids, yet have a modulus so low as to appear fluid in some regards.

As polymers are able to take on an enormous range of internal conformational arrangements, due to their flexibility, dynamical properties of polymers are determined primarily by their largely one-dimensional topological nature (internally, connectivity between segments dominates) and the presence of restrictions formed by neighbours in the material (externally, molecules can not pass through one another). Given this, the physics of complex fluids takes aspects from many fields, including statistical mechanics, thermodynamics and fluid mechanics. In this thesis, we will handle the rheology of polymeric fluids as given rise to by the 'tube' model and the dynamical notion of 'reptation' 
proposed by De Gennes [1], and expounded further in the text by Doi and Edwards [2]. We set out to examine the rheology of complex fluids [3] from the molecular standpoint, and in particular, nuclear magnetic resonance spectrocopy will be used as our primary investigative tool.

\subsection{Magnetic Resonance in Materials}

The invention of nuclear magnetic resonance was the discovery that radiofrequency electromagnetic radiation interacts with the nuclei in materials in ways that are dominated by the intrinsic properties of the species of nucleus involved - mass, charge and spin. More than this though, there is finer structure to this interaction arising from the environment in which those nuclei reside, which yields an astonishing amount of new information, valuable in particular to the chemist and in medicinal areas.

Upon application of a 'strong' polarising magnetic field, the nuclear magnetic moment interacts by precessing about this imposed axis - all the information that can be gleaned is a result of detecting the frequencies of this precession. The detailed structure that comes about through various mechanisms: electrons in molecular orbitals are driven by the external field and hence perturb the field felt by the nucleus; through space, one nuclear magnetic moment influences another; the response of two paired nuclei can be decided by the orientation of the internuclear vector with respect to the external field.

All of these effects contribute to the power of nuclear magnetic resonance to be of use in determining complicated chemical structures, but the real selling point for magnetic resonance in the wider community is that experiments can be designed that can specify where the nuclei are within the sample, and what they are doing while located there. Techniques that incorporate these spatially selective abilities are termed microscopic or imaging techniques, as opposed to spectroscopic techniques, though this does not prevent the combination of the two into an area unsurprisingly called spectroscopic imaging.

In conjunction with other aspects of magnetic resonance such as imaging and velocimetry, we aim to measure statistical molecular properties such as alignment under flow, in correlation with macroscopic observables such as the measured flow field itself. By doing so this thesis contributes to knowledge bridg- 
ing the gap between macroscopic rheological phenomena and the microscopic mechanisms from which such phenomena emerge.

\subsection{Introduction to this Thesis}

This thesis looks at a spectrum of complex fluids, ranging from cross-linked polymers (elastomers) and polymer melts, through to semi-dilute solutions of polymers and micellar structures, under flow. Using proton spin-spin relaxation as well as deuteron quadrupole spectroscopy, this thesis adds to previous research to demonstrate the degree to which molecular alignment occurs, under both shear and extensional flow arrangements, in a variety of interesting materials.

Chapter 2 provides a survey of soft condensed matter physics, a rich and marvellous field which primarily revolves around rheology, a hybrid topic utilising fluid mechanics, thermodynamics and statistical mechanics. Rheology is a large subject, with many fascinating aspects both in theory and experiment currently under investigation, and due to its size, this chapter calls upon only selected resources that are needed throughout the course of the thesis. These are mainly concerned with describing experimental geometries which provide idealised shear and extensional deformations of materials, in particular those materials which demonstrate non-Newtonian phenomena such as shear-thinning, shear-thickening and shear-banding.

In Chapter 3, the fundamentals of magnetic resonance are described. Like rheology, this field has a considerable history, yet remains in development both experimentally and theoretically. This thesis covers the basics of magnetic resonance spectroscopy (describing the nuclear Hamiltonian and relaxation mechanisms), imaging (using magnetic field gradients to encode spatial information into nuclear precession processes) and velocimetry (detecting displacement of nuclei over the course of an experiment), but goes on to describe how each subdiscipline of magnetic resonance can be incorporated into more complicated experimental techniques using two or more simple classes of experiment in fusion. As such, magnetic resonance becomes a crucial tool in the mechanically non-invasive toolbox of the rheologist. One tool of note described in this chapter which is used throughout this thesis is the Hankel transformation, of particular 
use in our work due to the cylindrically symmetric nature of the apparatuses used.

Chapter 4 describes a work in numerical simulation of the transverse relaxation of proton magnetic resonance coherences in polymer melts under shear. It uses as a foundation experimental work carried out by Cormier et al. which reported such relaxation times in sheared (poly)dimethylsiloxane, and uses the Ball-Callaghan-Samulski (BCS) model to numerically replicate the results. The BCS theory relies on the probabilities of monomer segments to return to their original region within the 'polymer tube', all-the-while undergoing diffusion. Correlation of residual nuclear dipole interactions can only be guaranteed in the case of return, and hence the known one-dimensional, curvilinear diffusion characteristics of polymers in 'tubes' is shown to contribute to a successful model of magnetic resonance tranverse relaxation. Such proton-based experiments are therefore shown to provide an option to the more prevalent deuteron-based investigations into molecular alignment.

Chapter 5 sees this thesis move from research on the polymer-dense melt phase into rheo-NMR investigations of polymer solutions, and in particular, a solution of a Pluronic triblock copolymer (polyethylene oxide-polypropylene oxide-polyethylene oxide) which self-assembles into wormlike micelles in a certain temperature range in the presence of a core-stabilising molecule. This chapter uses a wide range of magnetic resonance pulse programs to provide spectroscopic information, imaging, diffusometry and velocimetry to aid in the understanding of shear-banding phenomena. This sample in particular highlights the non-universal correspondence between regions of aligned micelles, and the zones of differing shear rate within the shear-banding phenomenon.

Where the bulk of rheology and rheo-NMR involves studies of the deformation of fluids in shearing geometries, Chapter 6 of this thesis explores the development of geometries that may be used to investigate the extensional aspects of the mechanics of fluids. The basic form of the apparatus used is the semi-hyperbolically converging die (SHCD), which a deliberately designed contracting pipe-flow device. The rate of contraction of the pipe is such that a constant extension-rate is provided to the sample, and as such we again use many rheo-NMR techniques to map out the flow field within the device, and in general investigate the applicability and practicality of magnetic resonance to 
demonstrate the complementary nature of shear and extensional deformations to materials. 


\section{Bibliography}

[1] PG DeGennes. Reptation of a polymer chain in presence of fixed obstacles. J. Chem. Phys., 55(2):572, 1971.

[2] M Doi and SF Edwards. The Theory of Polymer Dynamics. Oxford University Press, 1986.

[3] RG Larson. The Structure and Rheology of Complex Fluids. Oxford University Press, 1999. 


\section{Chapter 2}

\section{The Mechanics of Soft Materials and Polymers}

\subsection{What are soft materials?}

Soft condensed matter science is concerned with the investigation of the mechanical and molecular organisational properties of materials which lie in the vast space between the heavily idealised (and rarely perfectly realised) cases of inviscid fluids and immutable elastic crystal structures.

By covering such a wide range of conditions, the applicability of soft matter physics is equally broad. Nearly all biological systems are soft, as are all foodstuffs by corollary. Many soft materials are composed of multiple components of differing structure and behave like fluids at some times, and more like solids at others. Any material which will flow under certain conditions and maintain a persistent structure for at least some timescale can loosely be termed a complex fluid. Foams, emulsions, liquid crystals, micellar and colloidal materials are all examples of complex fluids [1]. The range of materials and technologies using them which fall into one of these categories is enormous: cosmetics, foodstuffs, petroleum products, pharmaceuticals, fire extinguishing materials, graphical displays, and paints; to name a few. There are obviously distinct specialist features in each application, though some common themes underpin their material design. One particularly common feature is that of each material's delivery to its intended target, in that it is desirable in many cases that 
the material flows easily towards its destination, before performing its function. Cosmetics and paints should, for example, be easy to distribute evenly over a surface, before becoming more rigid, in this case highlighting the need for a continual change of viscosity with the change in force applied by fingers, brush or spray can.

Through theoretical understanding of the behaviours of complex fluids, which are far from completely understood, new material properties are able to be predicted, controlled and engineered. Experimentally, mechanical deformation and response comes under the heading of rheology. A conventional mechanical rheometer can impart deformation to a sample, in a variety of geometries, in a variety of ways. A resultant stress can be found for a given deformational procedure, or a stress can be imposed which results in the observable deformation of the material. Besides the use of the rheometer, however, many alternative techniques are of immediate use to a soft matter scientist, largely due to the diverse nature of such materials and the requirements they impose on experimentalists. Magnetic resonance [2-4] has great potential for use in condensed matter systems (and in particular fluids); spectroscopic methods and the measurement of molecular motion provide insight regarding molecular organisation and dynamics, both key determinants of complex rheological behaviour. Optical methods [5] are very often of great assistance in the instance that the complex fluid is relatively transparent, which is the case for many solutions of polymers in water. Both of these alternatives incorporate the additional benefit of being non-invasive to the structure of a sample to a very large degree.

\subsection{Overview}

Here we discuss the requisite background material for the investigation of the response of soft materials when pushed or pulled. In the experiments to be seen later, various geometries are used to impart the appropriate deformation, most of which involve continuous flow.

For this reason, the following chapter discusses the ideas unifying the two extremes of soft materials - fluids that are elastic and in some sense solid-like, and soft solids that have such low bulk-modulus so as to appear liquid-like by taking on the shape of a restricting 'container'. We discuss soft materials often 
coming at the problem from two opposing points of view.

The 'liquid vs. solid' approach is one example of such a dichotomy. Another is the difference between specifying mechanical and dynamical quantities within materials as differential and continuous values such as tensors valid at a point, yet in reality materials are composed of vast numbers of discrete molecular components. In a similar vein, the description of the motions of fluids in a region can be done in two ways [6]: a Lagrangian expression of a flow is made by the specification of velocities of particular volume elements as they undergo motion over time; the Eulerian method of flow description is to specify the velocity of fluid as a function of space, no matter which fluid element may reside at such a position.

The basics of rheology associated with driving our samples through and around our experimental geometries will be discussed first in this chapter. This consists predominantly of describing the Couette system and the constantextension-rate cell. This semi-hyperbolically converging die will often be abbreviated to SHCD from here onward. The second portion of the chapter will investigate polymer-like structures, and how the microscopic dynamics of such materials influences their macroscopic rheological properties.

\subsection{Deformation of continuous media : stress and strain; viscosity and elasticity}

If a portion of material were to sit at rest at constant temperature for a 'long time', say, on a bench or in a beaker, it would be said to be in thermal equilibrium, once no further changes are observed. Any structural changes that could occur after a temperature change have occurred already, and there are no 'internal forces' or internal stresses at work upsetting the balance.

\subsubsection{Deformation and velocity gradient tensors}

At this point, if the material is deformed, there are immediately various quantities we can define without knowing anything about the chemistry or structure of the material, except that the co-ordinates, $\{\mathbf{r}\}=\{(x, y, z)\}$ of each material point at time, $t$, may have been shifted in a 'smooth' way to new co-ordinates, 
$\left\{\mathbf{r}^{\prime}\right\}=\left\{\left(x^{\prime}, y^{\prime}, z^{\prime}\right)\right\}$ at time, $t^{\prime}$. The tensor representing this transformation, $\mathbf{E}$, such that $\mathbf{r}^{\prime}=\mathbf{E} \cdot \mathbf{r}$, defined at every point over the original volume, is known as the deformation tensor. The deformation tensor's components are defined as a function of the two times, $t$ and $t^{\prime}$,

$$
\begin{gathered}
E_{i j}\left(t, t^{\prime}\right)=\frac{\partial r_{i}^{\prime}}{\partial r_{j}} \\
\mathbf{E}\left(t, t^{\prime}\right)=\left(\begin{array}{lll}
\frac{\partial x^{\prime}}{\partial x} & \frac{\partial x^{\prime}}{\partial y} & \frac{\partial x^{\prime}}{\partial z} \\
\frac{\partial y^{\prime}}{\partial x} & \frac{\partial y^{\prime}}{\partial y} & \frac{\partial y^{\prime}}{\partial z} \\
\frac{\partial z^{\prime}}{\partial x} & \frac{\partial z^{\prime}}{\partial y} & \frac{\partial z^{\prime}}{\partial z}
\end{array}\right)
\end{gathered}
$$

The variation of the local velocity of sample in motion at points within the material may be expressed by the velocity gradient tensor. As a function of the location of the point, its nine components, $\partial v_{i} / \partial r_{j}$, describe the variation of three independent components of velocity, with respect to three basis coordinates - given as follows in Cartesian space:

$$
\nabla \mathbf{v}=\left(\begin{array}{ccc}
\frac{\partial v_{x}}{\partial x} & \frac{\partial v_{x}}{\partial y} & \frac{\partial v_{x}}{\partial z} \\
\frac{\partial v_{y}}{\partial x} & \frac{\partial v_{y}}{\partial y} & \frac{\partial v_{y}}{\partial z} \\
\frac{\partial v_{z}}{\partial x} & \frac{\partial v_{z}}{\partial y} & \frac{\partial v_{z}}{\partial z}
\end{array}\right)
$$

The symmetric part of the velocity gradient tensor, $(\nabla \mathbf{v})_{i j}$, the rate-ofdeformation tensor, $\mathbf{D}$, can be written as

$$
2 \mathbf{D}=\nabla \mathbf{v}+(\nabla \mathbf{v})^{T}
$$

or,

$$
D_{i j}=\frac{1}{2}\left(\frac{\partial v_{j}}{\partial r_{i}}+\frac{\partial v_{i}}{\partial r_{j}}\right)
$$


There is geometrical distinction between elements of $\mathbf{E}, \nabla \mathbf{v}$ and $\mathbf{D}$ that lie on- and off-diagonal. Diagonal elements in each case represent deformations or relative motions of two infinitesimally close points in materials which are made along their separation vector, and known as extensional phenomena. In complement to that, off-diagonal elements represent cases for which two points are made to move relatively in directions normal to their separation vector, and are collectively known as shearing phenomena.

In either case, it is not unsurprising that the total deformation achieved is an integral of the velocity gradient history. By the chain rule and using definitions 2.2 and 2.4 ,

$$
\frac{\partial}{\partial t} \mathbf{E}=\frac{\partial \dot{\mathbf{r}}}{\partial \mathbf{r}^{\prime}}=\frac{\partial \mathbf{r}}{\partial \mathbf{r}^{\prime}} \cdot \frac{\partial \dot{\mathbf{r}}}{\partial \mathbf{r}}=\mathbf{E} \cdot \nabla \mathbf{v}
$$

\subsubsection{Stress}

The mechanical forces on the faces of a cubic volume element arising from its surroundings can be broken into tangential and normal components, as shown in fig.2.1.

These eighteen values are not independent [7]. In the infinitesimal volume limit the normal force on one face must be exactly balanced by that normal force on the opposing face, else infinite accelerations occur due to a force proportional to $d^{2}$ acting on a volume proportional to $d^{3}$ - nullifying any difference in force in an infinitessimally short time. A similar argument holds to show that imbalances between $\sigma_{i j}$ and $\sigma_{j i}(i \neq j)$ are also eliminated. This symmetry reduces the number of independent parameters of the stress tensor to six: in a Cartesian system these are namely the three normal forces along the three axes and three shear stresses acting parallel to the faces of an infinitesimal volume under investigation.

Effects of deformations that cause uniform strain to be present throughout the material give rise to the linear constitutive equation, relying on the additivity of successive changes in strain (as is well explained in several texts $[8,9]$ )

$$
\sigma_{i j}=\int_{-\infty}^{t} G\left(t-t^{\prime}\right) D_{i j}\left(t^{\prime}\right) d t^{\prime}
$$




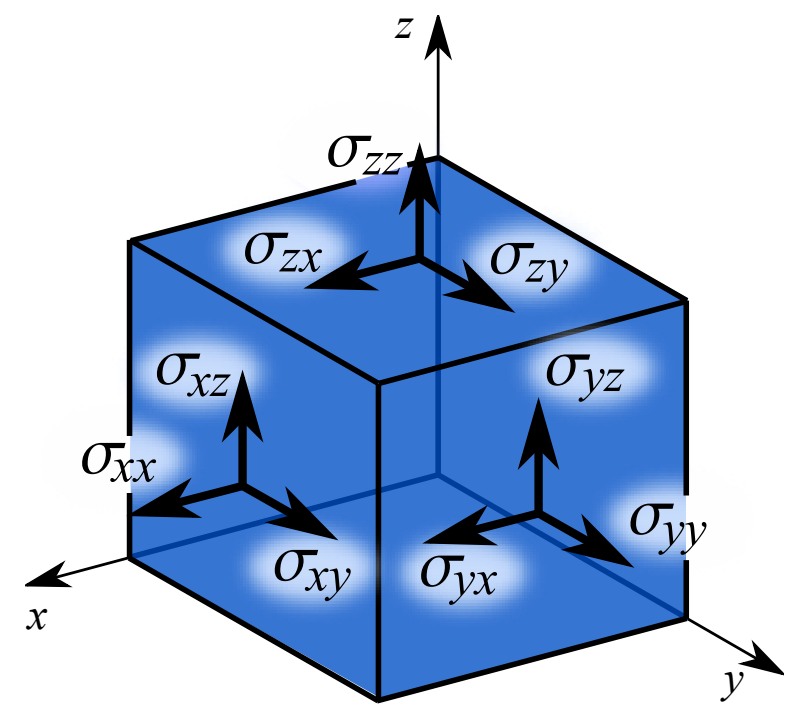

Figure 2.1: The components of the stress tensor and their relation to the forces on a unit volume.

where $G(s)$ is the stress relaxation modulus, and represents the remanent stress remaining at time $s$ following a particular step strain.

\subsubsection{Shear viscosity}

Upon a deformation applied parallel to the surface of a material, matter within the bulk, yet connected to the surface in some way - whether through loose or strong bonds, or through molecular collisions - will respond through the transfer of momentum. In a simple fluid the propensity of the material away from the surface to follow the motion of the surface is known as its viscosity, and is labelled $\eta$. Viscosity can conversely, and slightly more intuitively, be viewed as the resistance of a material to flow and how much force is required to establish a velocity gradient.

Consider that a uniform velocity gradient has been established in a parallelplate device in which one plate (whose normal lies in the $y$-direction) is moving at uniform speed (in the $x$-direction); a plate at rest is bounding the opposite surface (these might be considered the top and bottom faces of the volume depicted in fig.(2.2)). The steady-shear viscosity is simply characterised by dividing the shear stress $\sigma$ within the sample (uniform in a simple fluid) by the shear strain rate $\dot{\gamma}$. It should be noted that $\dot{\gamma}$ may be associated with the 


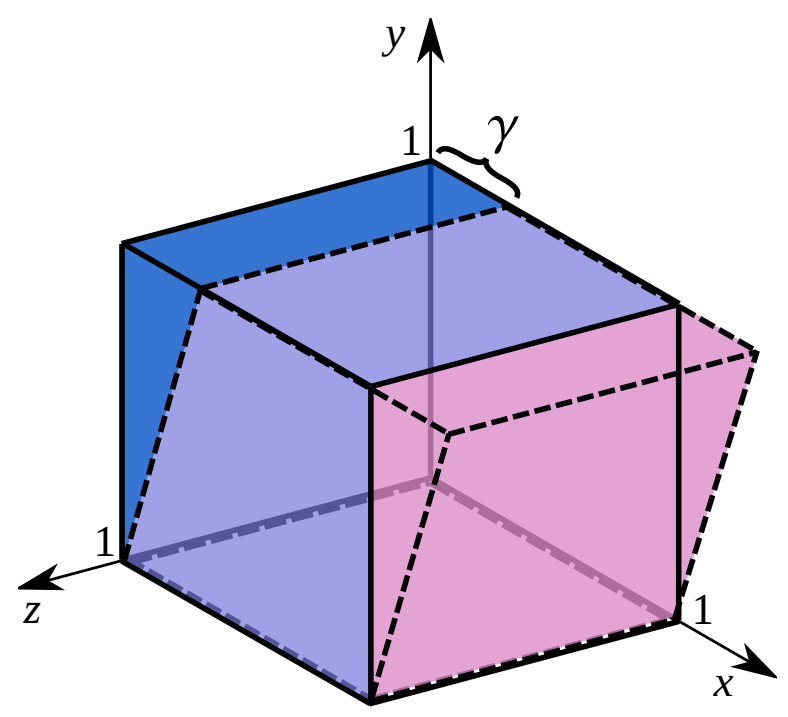

Figure 2.2: Visual representation of a shear deformation of a unit volume. The distance that (for example) the $y=1$ face of a cube of unit dimensions is displaced in the $x$ direction is called the strain component $\gamma_{y x}$

appropriate off-diagonal rate-of-deformation tensor component, $\dot{\gamma}=2 D_{y x}$. The steady-state shear stress/strain relationship then reads

$$
\sigma_{y x}=\dot{\gamma} \eta_{0}
$$

where $\eta_{0}$ is the steady-state shear viscosity corresponding to the experiment carried out at a shear-rate arbitrarily close to zero. This value would, in practice, be obtained by making an extrapolation to zero-shear after several non-zero shear rates have been applied.

Rheology carried out in such a way as to extract this information would normally be performed within a cyclic geometry due to the practical constraints imposed by the need for unbounded motion to ensure steady-state conditions. In these geometries, experiments which obtain the steady-shear viscosity under large, unbounded deformation, would be termed non-linear rheology. Samples that retain the same measured viscosity no matter the strain rate applied are known as Newtonian, and conversely those for which viscosity is strictly a function of strain rate are known as non-Newtonian. 


\subsubsection{Linear viscoelasticity}

By making small oscillatory deformations to a sample, linear rheology measurements may be carried out. In such experiments two aspects of material properties become apparent: their viscosity and their elasticity. Where viscosity is the property related to the ease with which material will flow under a shear strain, the elasticity can be associated with its shape memory, in other words the degree to which the material would like to spring back to its original form. For this reason, these two properties are called the loss and storage moduli, respectively: viscous effects tend to dissipate energy (loss), while energy is stored in elastic deformation, so the material can respond reversibly.

Substituting the shear strain rate determined by the time-derivative of the oscillatory shear strain, $\gamma=\gamma_{0} \sin \omega t$, into equation 2.8 ,

$$
\begin{aligned}
\sigma(t) & =\int G(s) \omega \gamma_{0} \cos [\omega(t-s)] d s \\
& =\gamma_{0}\left[\omega \int_{0}^{\infty} G(s) \sin \omega s d s\right] \sin \omega t+\gamma_{0}\left[\omega \int_{0}^{\infty} G(s) \cos \omega s d s\right] \cos \omega t \\
& =\gamma_{0}\left[G^{\prime}(\omega) \sin (\omega t)+G^{\prime \prime}(\omega) \cos (\omega t)\right]
\end{aligned}
$$

that is to say that elastic effects quantified by $G^{\prime}$ are in phase with the deformation and viscous effects quantified by $G^{\prime \prime}$ are out of phase with the strain. In non-Newtonian fluids these quantities are intrinsically strain-frequency dependent and reflect timescales over which structures within the material both form and relax to equilibrium. The cross-over between $G^{\prime}$-dominated stresses and $G^{\prime \prime}$-dominated stresses is located at an oscillation frequency commensurate with the longest timescale over which structural relaxation occurs, and hence the cross-over between the material being solid-like or liquid-like. This variability of behaviour in materials demonstrates the "fuzziness" so important to soft matter physics; all materials flow on some timescale.

$G^{\prime}(\omega)$ and $G^{\prime \prime}(\omega)$, as defined as functions of the frequency of oscillatory shear give insight into the timescales over which structures relax (return to minimal internal stresses). The Maxwell fluid forms the simplest model of viscoelastic behaviour, and does so by positing a single relaxation time at work 
in a material. It incorporates two distinct behaviours: one attributable to a Hookean solid (perfectly elastic, storing energy) and a Newtonian liquid (perfectly viscous, dissipating energy). These two modes are often portrayed by the simple resistor-capacitor (RC) electrical circuit, or analogously by the springand-dashpot system, shown schematically in fig.(2.3).

The storage and loss moduli in this model, with single relaxation time, $\tau$, are given by

$$
G^{\prime}(\omega)=\frac{G_{0} \omega^{2} \tau^{2}}{1+\omega^{2} \tau^{2}} \quad G^{\prime \prime}(\omega)=\frac{G_{0} \omega \tau}{1+\omega^{2} \tau^{2}}
$$

and it can be noted that $\frac{G^{\prime}}{G_{0}}-\left(\frac{G^{\prime}}{G_{0}}\right)^{2}=\left(\frac{G^{\prime \prime}}{G_{0}}\right)^{2}$. Completing the squares appropriately, and using the frequency at which the moduli are measured as a parameter, a plot of $G^{\prime \prime}$ vs $G^{\prime}$ gives a semi-circle

$$
\left(\frac{G^{\prime \prime}}{G_{0}}\right)^{2}+\left(\left(\frac{G^{\prime}}{G_{0}}\right)-\frac{1}{2}\right)^{2}=\frac{1}{4}
$$

as shown in fig.2.3. This so-called Cole-Cole plot (which depicts the two moduli normalised by the plateau modulus $G_{0}$ ) is useful in graphically indicating the frequency for which linear rheology on a sample causes deviation from the Maxwell model. To first order, many complex fluids (worm-like micelle solutions, for example), exhibit Maxwellian behaviour. Deviations occur, however, and are often seen through the appearance of multiple relaxation rates within the material.

\subsubsection{Simple examples of non-linear rheology in materi- als}

When complex fluids are subjected to continuous deformation, the mechanical response may depend on applied strain rate, an effect known as non-linear rheology. From common experience, several properties of non-Newtonian fluids are evidently possible. A material which is seen to be a thick liquid or soft solid in equilibrium may shear thin, in which the viscosity decreases with shear rate. Conversely, some materials such as a cornstarch solution become more 

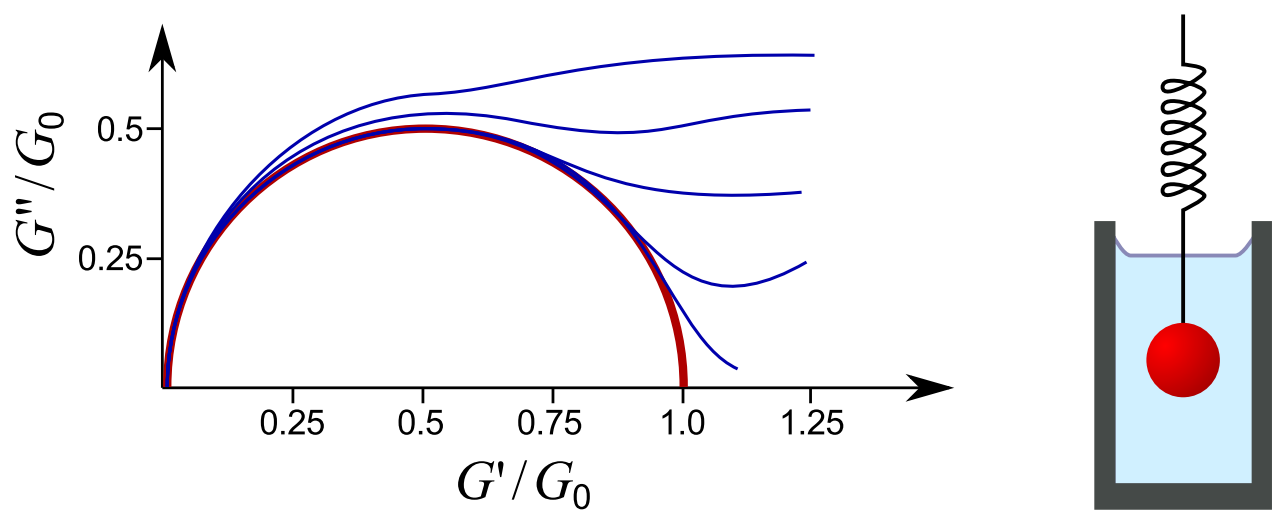

Figure 2.3: (left) A schematic Cole-Cole plot showing ideal Maxwellian behaviour (bold, red semicircle), along with the corresponding curves for five different samples. This plot-type demonstrates quite clearly at which oscillating shear frequency a sample deviates from ideal monoexponential behaviour. The plateau modulus, $G_{0}$, reached by $G^{\prime}$ is used to normalise both $G^{\prime}$ and $G^{\prime \prime}$ in this format, and (right) The spring-and-dashpot analog of the Maxwell fluid, incorporating independent perfect storage and loss components, which generate a monoexponential decay of oscillatory amplitude.

viscous with increasing shear rate. Figure (2.4) demonstrates some simple cases of materials, incorporating intuitive ideas such as yield.

\subsubsection{Dimensionless parameters}

With complex materials displaying variable behaviours under different conditions or at different times, the use of dimensionless numbers characterising the experimental parameters, in relation to material properties becomes convenient. In rheology one of the most important of these is the Deborah number, De, defined as the quotient of the material's terminal mechanical relaxation time $\left(\tau_{R}\right)$ and a time constant characteristic of the flow $\left(\tau_{\text {expt }}\right)$, such as the oscillation period in linear rheology

$$
\mathrm{De}=\frac{\tau_{R}}{\tau_{\text {expt }}}
$$

In special cases the appropriate dimensionless number characterising the flow takes other names: the Weissenberg number uses the inverse of the shear rate as the characteristic time of deformation in non-linear rheology, 


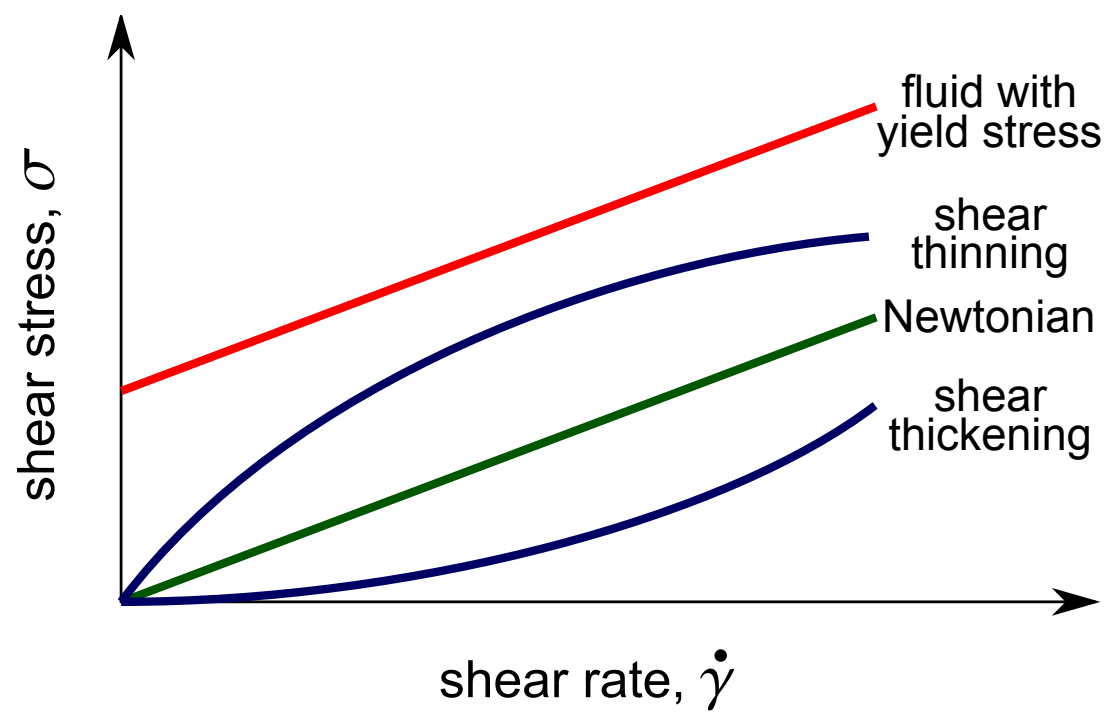

Figure 2.4: Examples of some of the simplest behaviours of fluids: shear thickening (apparent viscosity increase with shear), Newtonian (constant viscosity for all shear rates), shear thinning (apparent viscosity decrease with shear rate), yield-stress fluid (requires stress in excess of some threshold before flow occurs)

$$
\mathrm{Wi}=\dot{\gamma} \tau_{R}
$$

and the Peclet number concerns the advection of material, being formed by the ratio of the rate of motion due to flow with the rate due to diffusion

$$
\mathrm{Pe}=\left(\frac{v}{a}\right) /\left(\frac{D}{a^{2}}\right)=\frac{v a}{D}
$$

where $a$ is a characteristic distance to be travelled by either mechanism, $v$ is the average velocity due to flow, and $D$ is the diffusion coefficient of the molecules in motion.

The use of dimensionless parameters is of greatest use in delineating experimental regimes. Situations in which a material appears to act in a solid-like or liquid-like way are described by De $\gg 1$ and De $\ll 1$. Despite the variability of relaxation dynamics in a wide range of materials, bulk material dynamics under flow conditions can be treated similarly when such ratios are introduced, as in the familiar cases of Mach number (ratio of flow velocity to the velocity of sound within the material) and Reynolds number (ratio of the product of flow 
velocity and characteristic dimension to viscosity). In both cases the material behaves in a qualitatively different way if these numbers vary either side of a certain threshold, which is often unity (or can be made unity via scaling).

\subsubsection{Typical shearing geometries}

\section{Cone-and-plate}

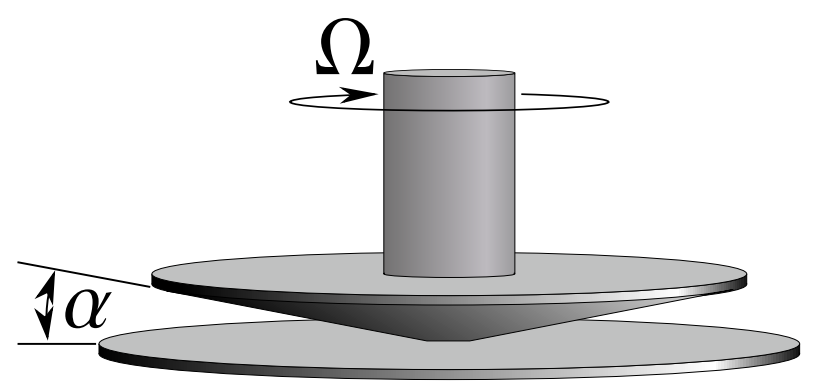

Figure 2.5: A uniform shear rate field can be generated by a cone with inclination angle $\alpha$ rotating at uniform angular frequency $\Omega$

The cone-and-plate geometry in which a sample sits in the vacant space between an inverted cone above, and horizontal plate below, shown in fig. 2.5, is well-known in the rheological community. Its main virtue is the presence of a uniform shear rate at all positions within the sample volume, as well as being able to detect normal stresses generated by deformed samples. While the horizontal plate remains stationary, the upper conical surface is turned about its axis in an oscillatory fashion such that the angular position satisfies $\Omega(t)=\Omega_{0} \cos \omega t$, generating oscillatory shear flow. With the shear rate being proportional to the wall velocity and inversely proportional to the gap size, the inclination of the cone face implies the gap size increase linearly with radial displacement, which is just what is needed to balance the increasing wall velocity determined through elementary angular mechanics, maintaining a constant shear rate of $\dot{\gamma}=\tan \alpha$, where $\alpha$ is the cone angle. At small strain amplitudes, $\gamma_{0}=\Omega_{0} /(\omega \tan \alpha) \sim 0.02$, structural relaxations occur according to the material's linear response and corresponding stresses can be measured. 


\section{Cylindrical Couette geometry}

Where there are advantages to the cone-and-plate rheological geometry, the few weaknesses that exist are adequately taken care of at very little cost by the Couette (or concentric cylinders) geometry, schematically shown in fig. 2.6. By placing sample within the gap formed in the intervening space between two closed-bottomed tubes, with one of the two tubes (usually the inner) to be rotated continuously, a shear field is generated. By controlling the rate at which the cylinders are rotated with respect to one another, a strain-rate controlled experiment is carried out at strain rates $\dot{\gamma} \sim V / d$, where $V$ is the relative velocity of the walls, and $d$ is the distance between them. The relationship is only exact in the limit of infinite parallel plate Couette geometry, however if the no-slip boundary condition holds (fluid velocity at the wall is precisely the velocity of the wall), then the average strain rate may be written

$$
\dot{\gamma}_{\mathrm{av}}=\frac{V}{d}
$$

Stress measurement (and stress controlled experiments) are mechanically possible in conventional rheometers, however in the case of the magnetic resonance measurements carried out later in this thesis, internal stress can only be indicated indirectly through molecular properties such as alignment.

The main advantages to this geometry are that the containment of the sample is much greater, especially for low viscosity fluids such as water, but also for all fluids being deformed at reasonably high Deborah number; and the greater volume able to be actively investigated under shear. Conversely, the main disadvantage is the slight lack of uniformity of the local stress within the sample, dictated by the conservation of angular momentum at differing radii. Minimising the gap size will obviously reduce this variance, as will increasing the Couette cell radius, i.e. making the gap look more and more like the ideal 'infinite parallel plates' geometry. The stress values between the walls is non-uniform, and may be written

$$
\sigma_{y x}(r)=\sigma_{\mathrm{ow}} \frac{r_{\mathrm{ow}}^{2}}{r^{2}}
$$

where $\sigma_{\text {ow }}$ and $r_{\text {ow }}$ are the stress and radial position at the outer wall, respec- 
tively.

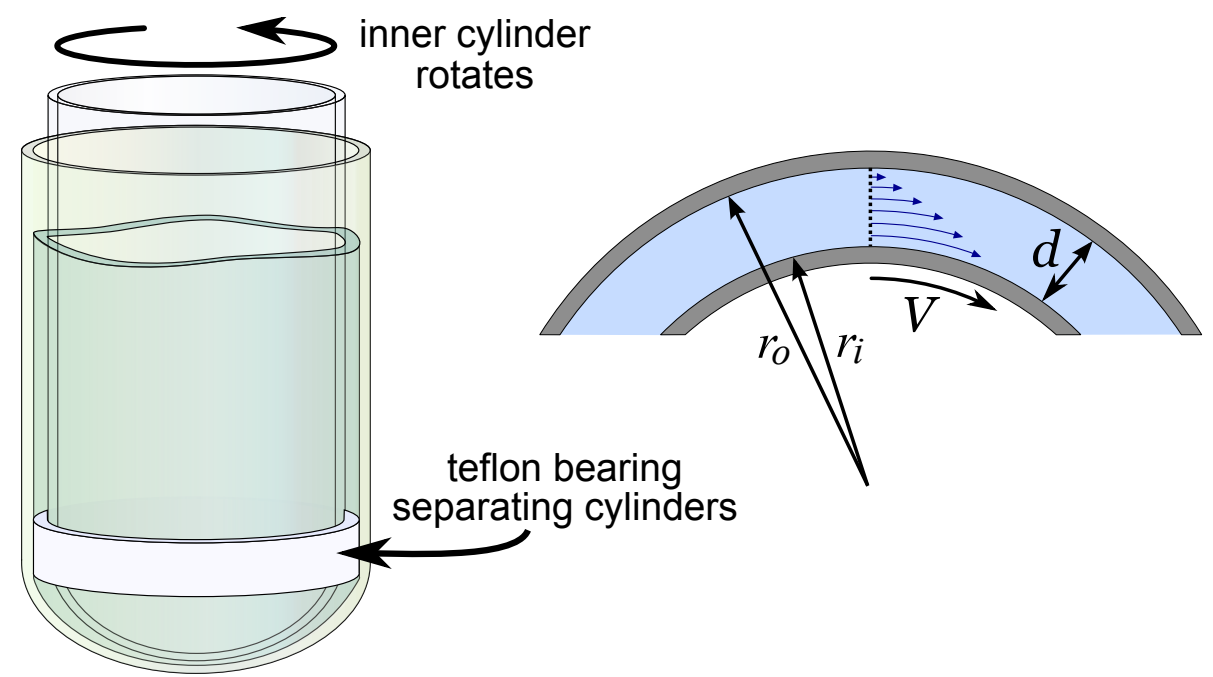

Figure 2.6: A cylindrical Couette geometry, generating a shear field on a fluid lying between two cylinders. The shear rate is determined by the diameters of the tubes and the rotation frequency of the inner tube. The stress is nonuniform, being inversely proportional to the square of radial position, though this effect can be reduced through the appropriate choice of cylinder radii.

\subsubsection{Extensional deformation}

As seen in the previous section, the behaviour of polymers and other complex fluids can be explored in a routine way by conventional rheometers equipped with well-described geometries. In characterising materials, however, it should be kept in mind that such geometries are, to various degrees, idealised to measure the shear stresses and strains. Via processes such as extrusion or film blowing, very different mechanical properties may be observed than those expected purely through shear flow mechanical responses [10, 11]. Such phenomena indicate that conventional rheology, while giving a near total description of material properties, is insufficient to completely characterise complex fluids.

Extensional studies of viscoelastic materials are complementary in nature to those which focus on pure (or close to pure) shearing flow properties. Extensional deformation involves applying stresses to a sample in which the forces acting on infinitesimal volumes are normal to all of the faces of that volume, and as such they have a stretching or compressing effect on the sample. 


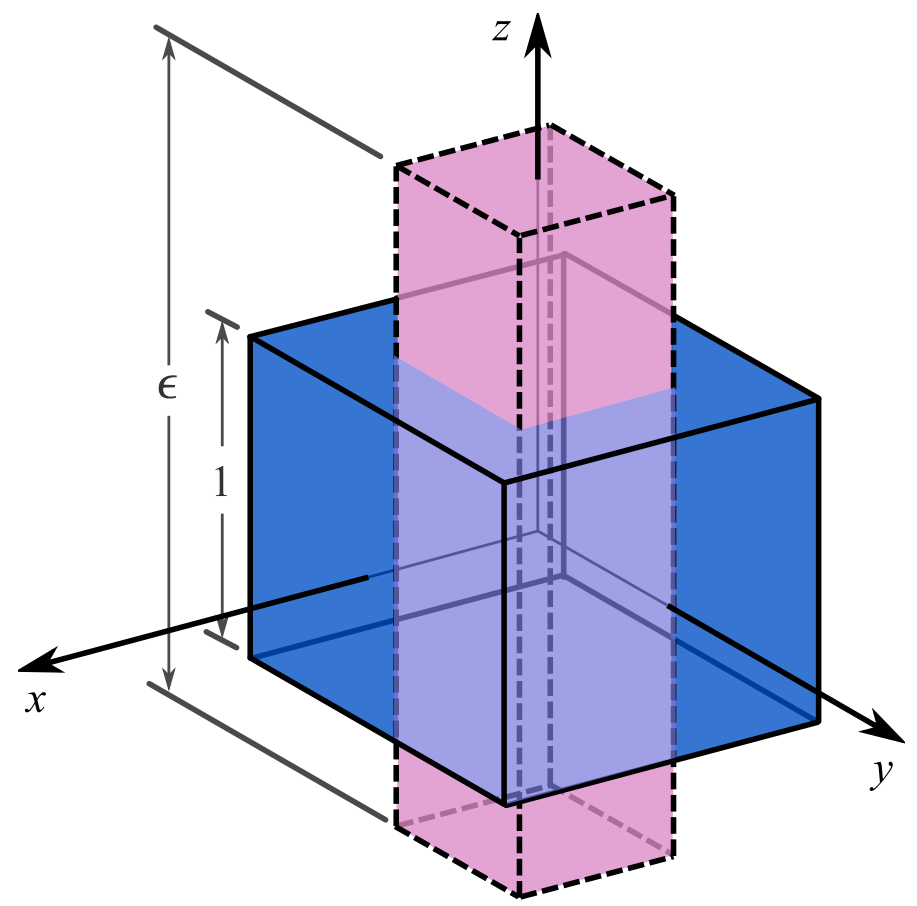

Figure 2.7: The components of the stress tensor and their relation to the forces on a unit volume.
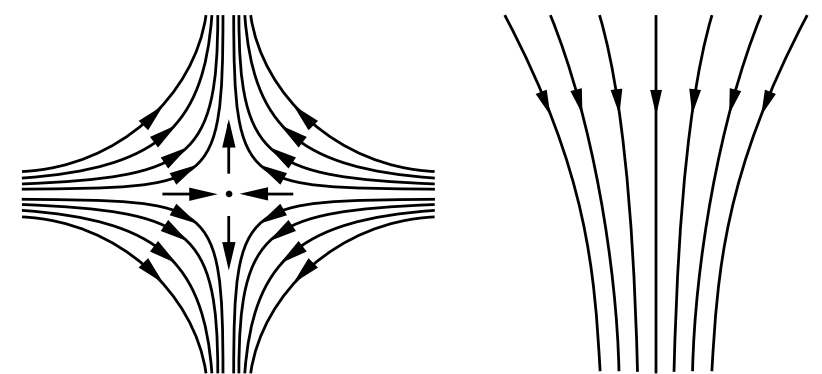

Figure 2.8: The flow field for an extensional flow is usually established in either an "opposed jet" type geometry (left) or a "filament stretching" type geometry (right). While the former allows continual flow (in mill apparatuses), a molecule following a streamline will not experience the pure extension that only the stagnation point offers. The converging streamlines of the latter make for a nearly global extension field, and a molecule moving along a streamline has a more well-defined strain history. The Lagrangian approach of imposing flow conditions on individual molecules rather than the flow field applies only in the second case.

Practically, an extensional flow experiment may only be constructed with one of either Eulerian (specify a velocity field as a function of fixed sample 
co-ordinates) or Lagrangian (specify the velocity of particles over the course of their travels) flow descriptions in mind - see section 2.2. From the viewpoint of this thesis it would be preferable to generate flows which are purely extensional after taking a Lagrangian viewpoint, as the dimensionless parameter formed by the product of the extension rate of a polymer and its tube disengagement time, no matter its position, should be constant. There are two main aspects of importance in the design of practical geometries to perform such experiments, and compromise in either one can make analysis and the validity of results questionable, however these problems may not be inhibitory to determining quantities that reflect a material's extensional properties akin to those found under shear. Firstly, it is rather more difficult to produce a local environment which is pure in extensional deformation than it is to produce an arbitrarily uniform shear field as in the Couette cell. Secondly, even where producing a finite region of pure extension is achieved, such extension cannot possibly continue indefinitely: with the assumption of affine deformation (and even without it in all but extreme cases) one dimension of the sample being monitored must very quickly (exponentially) increase and exceed the apparatus size in order to maintain a constant extension rate. Correspondingly, another independent dimension must diminish, and perpetual "steady state" extensional flow can not be achieved due to this. As such extensional rheology in a Lagrangian sense is a necessarily transient technique macroscopically, and is necessarily transient microscopically in an Eulerian sense. Whichever approach is taken, some measure of consistency and convention is required to enable comparison of results between experiments.

\subsubsection{Extensional viscosity}

Extensional viscosity is a material parameter that was largely unquantified before flow experiments in the early $20^{\text {th }}$ century. It was first made clear by the independent works of both Fano [12] and Trouton [13]; Trouton investigated a property of materials he called "the coefficient of viscous traction". Understanding of the fundamentals of the field was largely auxiliary to both of their original investigations, though: Trouton aimed to obtain the viscosity of Newtonian fluids, while Fano was interested in their spinnability, or thread-forming capabilities. 
Extensional flow of materials undergoing steady rate-of-strain can be described by a diagonal rate-of-strain tensor (which is equal to the velocity gradient tensor in this case). In the uniaxial extensional deformation case for which the velocity gradient condition $\frac{\partial v_{z}}{\partial z}=\dot{\varepsilon}$ holds, this results in the following rateof-deformation tensor, $\mathbf{D}$, in the Cartesian frame (extension in the $z$-direction):

$$
2 \mathbf{D}=\nabla \mathbf{v}+(\nabla \mathbf{v})^{T}=\left(\begin{array}{ccc}
-\dot{\varepsilon} & 0 & 0 \\
0 & -\dot{\varepsilon} & 0 \\
0 & 0 & 2 \dot{\varepsilon}
\end{array}\right)
$$

We may recall equation 2.7, showing the evolution of the deformation matrix as the result of a known imposed velocity gradient,

The deformation tensor, E, being the time-integral of itself multiplied by the velocity gradient tensor (see eqn. 2.7) may be written

$$
\frac{\partial}{\partial t} \mathbf{E}=\mathbf{E} \cdot \nabla \mathbf{v}
$$

and, if the deformation tensor at time zero is $E_{i j}(0,0)=\delta_{i j}$, in the case of purely extensional flow ( $\mathbf{D}$ and $\nabla \mathbf{v}$ are diagonal and therefore equal), it remains diagonal for all times, $t$. The time-dependence of the deformation tensor as calculated via equation 2.19 therefore provides three equations expressing coordinates of the sample. In particular, with the use of the velocity gradient tensor derived from equations 2.18 ,

$$
\begin{aligned}
\frac{\partial E_{x x}}{\partial t} & =-\frac{\dot{\varepsilon}}{2} E_{x x} \\
\frac{\partial E_{y y}}{\partial t} & =-\frac{\dot{\varepsilon}}{2} E_{y y} \\
\frac{\partial E_{z z}}{\partial t} & =\dot{\varepsilon} E_{z z}
\end{aligned}
$$

which dictates that for steady extensional flow, the $z$-coordinate of a point within the sample must increase exponentially in time, while any transverse coordinate must decrease correspondingly at half the rate. The change in length of a characteristic piece of the sample is known as the Hencky strain, and we may identify $\dot{\varepsilon}$ as the Hencky strain rate. 
Where the steady-state shear viscosity is generally written as the long-time limit of the quotient of shear stress and shear rate, it has an "extensional viscosity" equivalent, $\eta_{\mathrm{E}}$,

$$
\eta_{\mathrm{E}}(\dot{\varepsilon})=\lim _{t \rightarrow \infty}\left[\frac{\sigma_{\mathrm{E}}(t, \dot{\varepsilon})}{\dot{\varepsilon}}\right]=\lim _{t \rightarrow \infty}\left[\frac{\sigma_{z z}-\sigma_{x x}}{\dot{\varepsilon}}\right]
$$

where $\eta_{\mathrm{E}}(\dot{\varepsilon})$ is a (potentially) extension-rate dependent extensional viscosity, and $\sigma_{\mathrm{E}}$ is the "net tensile stress", $\sigma_{z z}-\sigma_{y y}$. As a long-time limit property, the extensional viscosity intrinsically and experimentally generates difficulties. As equation 2.20 shows, the Hencky strain must be generated by a change in sample length which is exponential, to keep the extension rate constant over the course of an experiment. In following sections we shall see how this condition leads to fundamental geometrical restrictions in particular experimental apparatuses.

For a Newtonian fluid under deformational flow, and for non-Newtonian flows at rates of deformation low enough that to first order the constitutive relation between stress and strain is simply [14]:

$$
\boldsymbol{\sigma}=2 \eta \mathbf{D}
$$

where $\eta$ is the conventionally measured shear viscosity. Inserting values for $\sigma_{z z}$ and $\sigma_{x x}$ as calculated by equation 2.24, using the uniaxial deformation rate tensor of equation 2.18 ,

$$
\begin{aligned}
\eta_{E} & =\frac{\sigma_{z z}-\sigma_{x x}}{\dot{\varepsilon}} \\
& =\frac{2 \dot{\varepsilon} \eta-(-\dot{\varepsilon} \eta)}{\dot{\varepsilon}} \\
& =3 \eta
\end{aligned}
$$

The proportionality constant between the shear and extensional viscosities is known as the Trouton ratio, and takes the value 3 for the Newtonian constitutive relation under the assumption of uniaxial deformation; biaxial deformation will generate different values. Trouton ratios have been measured up to four orders of magnitude higher than this, though the transient nature of the extensional deformation and characteristics of various experimental geometries 
preclude exact and agreed values of extensional viscosity universally.

In non-Newtonian flow experiments of larger deformations, the relation between shear and extensional properties of materials will require not only the constitutive equation, but also the strain history - further complicating the lack of agreement between rheologists in the field using different experimental arrangements. Studies of materials undergoing extensional flow are complementary, however, to the bulk of rheological studies which investigate the effects of shear forces in complex fluids, and are vital in understanding the fluid mechanics taking place during modern polymer processing techniques, amongst other things.

\subsubsection{Conventional extensional rheometry}

The transient nature of extensional rheology makes extensional rheometry much more technically difficult than shear rheometry, and in particular, due to the arguments of Trouton, extensional rheometry is important in the regime far removed from linear viscoelastic behaviour. In samples solid enough to be clamped and stretched, upper limits on the possible strains imposed are dictated by the size of the rheometer, necking and other instabilities of the sample, and the adhesion to the extensional mechanism. In less viscous samples which rely on the flow field as the generator of deformation, turbulence and strain history play a large role in experimental design [15].

\section{Sample stretching rheometers and the four-roll mill}

Most techniques available for use in experiments revealing extensional properties are limited to only providing the uniaxial deformation scenario. One apparatus designed to do this is the Sentmanat extensional rheometer, which uses two adjacent, counter-rotating rollers to stretch a sample attached to both surfaces. Fitted to a rheometer designed primarily to measure shear behaviour, the torque on the drive-shaft corresponds to the extensional stress within the sample. A second common method, again restricted heavily by the dimensions of the apparatus, is the Münstedt tensile rheometer, which is typical of most extensional apparatuses that rely on a linear actuator type transducer rather than measuring a torque. This device works on the principle that at all times only 


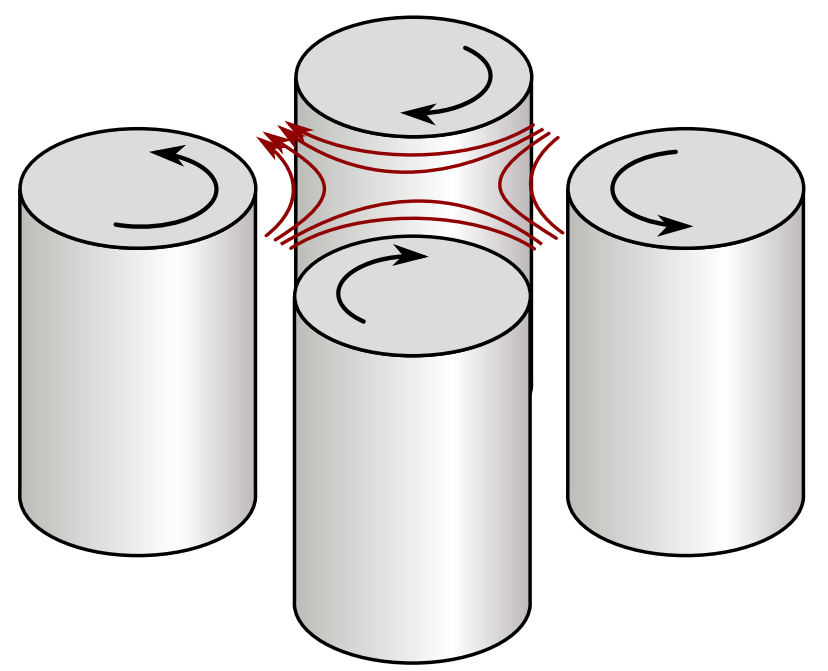

Figure 2.9: Schematic illustration of the four-roll mill. A stagnation point in the central volume is the location of a region of pure extension (for this configuration of rotating cylinders). Very high extension rates are made unrealistic by the inverse nature of the residence time of the sample within this volume. Strain history is also poorly defined as sample may follow a non-trivial route to the region of interest.

the ends of the material are being separated, though this means the technique relies slightly more on the quality of the clamping or gluing of sample to the two moving plates. Both these stretching devices, and other devices like them, have rather limited upper ranges in terms of extensional strain rate (typically between 5 and $10 \mathrm{~s}^{-1}$ ). By being applicable to samples which may be considered reasonably solid, however, the dimensionless parameter formed by the product of extension rate and terminal relaxation time can still be significant. Investigations of materials of lower bulk moduli can benefit from alternative geometries.

Complex fluids at the lower end of the viscosity spectrum may need to be contained in order to be meaningfully researched. A flow cell of some description can be used to generate extensional stresses in the same way that a Couette cell is designed to generate shear stresses purely via fluid mechanical mechanisms. The most ubiquitous of these is the four-roll mill (see fig.2.9), whereby a material can be locally deformed by four rotating cylinders which are located at the corners of a square. By making these cylinders rotate - alternating senses from roll to roll around the square - a region of local extension can be generated, stretching the sample in one direction, compressing in another. Such a geometry 


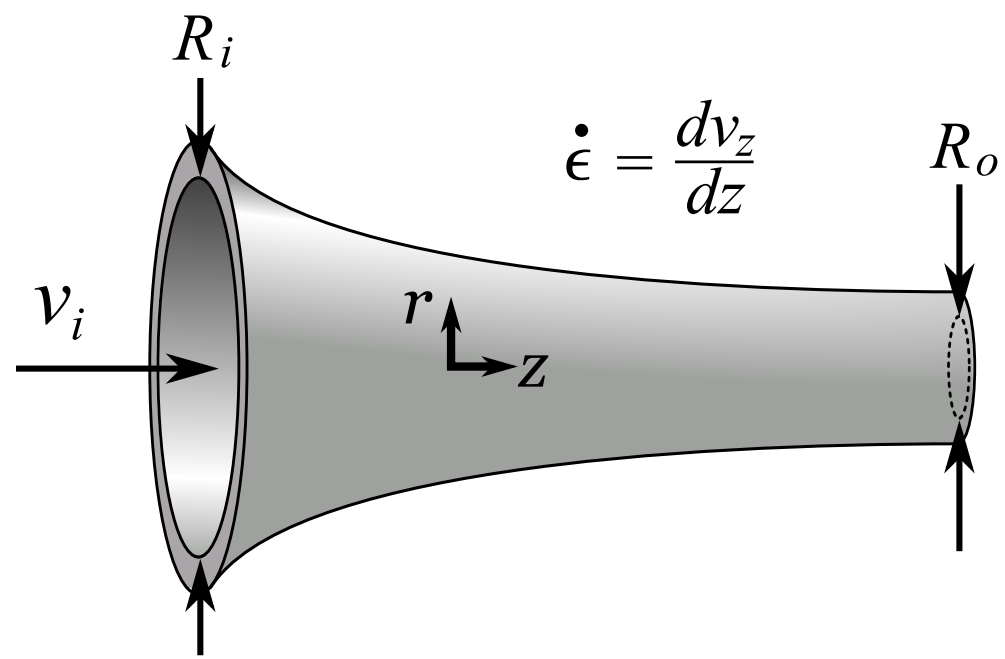

Figure 2.10: The semi-hyperbolic converging die, in which extensional stresses are overwhelmingly dominant along the centre of the pipe. Note that the direction of flow is collinear with the stretching direction, allowing a transient extensional experiment of longer duration

is versatile in the sense that regions of both nearly perfect extension and shear can be established in the same geometry. The main disadvantage to the design is that all portions of the sample spend only a relatively short time within the pure extension region, and that the geometry is not built from a Lagrangian standpoint, meaning that in a polymer sample, one molecule "passes through" an extensional region, rather than an extensional force "tracking" the molecule.

\section{Semi-hyperbolic converging die}

One solution to the demand for such a geometry is shown in fig. 2.10, and is known as the semi-hyperbolically converging die [16-19]. It is essentially a pressure-driven flow through a pipe of carefully varying radius, such that the extension rate is a constant, at least in a considerable region down the axis of the pipe, away from the walls. In the same way as the shear rate is constructed from knowing the relative motions of two fluid elements in the direction perpendicular to their relative displacements, the extension rate can be seen to be generated by the relative motions of two fluid elements in the same direction as their separation.

We explain the explicit form of this geometry in chapter 6 , in which we 
explore the SHCD as a viable geometry for Rheo-NMR experiments.

\subsection{Polymer Dynamics}

We now turn our attention to two examples of soft matter of specific relevance to this thesis. In this section we deal with the case of entangled linear polymer melts and semidilute solutions, and in the next, a closely related class of entangled "living" polymers formed from wormlike-micelle solutions.

Polymers are large, heavy molecules built up of small component parts known as monomers. In general, when we refer to polymers, they are treelike, in the sense that there are few or no loops, and the bulk of their mass lies in the carbon (or sometimes carbon/oxygen or silicon/oxygen) backbone. While it is possible to produce a plethora of topologies of polymers which are not curvilinear and one-dimensional, in this thesis we shall almost exclusively deal with polymers and polymer structures which are both highly flexible and pseudo-linear in nature. As such, much physical modelling and parametrisation can be done to simplify their description in many phases, whether isolated in a solution of choice, whether they are closely packed enough to weakly interact, or are packed more densely so as to entangle and become individually indistinguishable. In the first place we need to describe the freedoms of monomers segments in general, the results of which determine the expected size of the polymer and the timescale over which the fastest variations in conformation allow the shape of the polymer to change. At the other end of the dynamics scale is centre of mass motion, which is of little consequence in dilute solutions, but becomes vital when we investigate the diffusion of the polymer throughout a network as the mechanism for the longest stress relaxation characteristic time.

\subsubsection{End-to-end vector distribution}

As a polymer is comprised of much smaller linear components chemically joined, the position and orientation of one monomer is somewhat dependent on its neighbour. The range of freedom belonging to a monomer may be influential in the description of the overall macromolecule, but first we imagine that such bonds may be completely randomly directed. If we assume that monomers 
are identical in length, considering the end-to-end vector representing the displacement of the last monomer unit with respect to the first is the same as the classical stochastic problem of the random walk in three dimensions. With this in mind, a first guess at a distribution of end-to-end vectors $\{\mathbf{R}\}$ of widely separated polymers might bear the following signature:

$$
<\mathbf{R}>=0,<\mathbf{R}^{2}>=N b^{2}
$$

where $N$ is the number of monomers in the chain, and $b$ is the length of the monomer. This is called the freely-jointed chain model, and as it turns out, it is of the same form as the statistical distribution even when short-range interactions preventing total monomer freedom are included. Hence we can for the time being ignore limitations in flexibility, or even assume a distribution in monomer length, and write the probability of finding an end-to-end vector, $\mathbf{R}$, as

$$
p(\mathbf{R})=\left(\frac{3}{2 \pi b^{2}}\right)^{3 / 2} \exp \left(-\frac{3 \mathbf{R}^{2}}{2 b^{2}}\right)
$$

where we have just assumed that there is some effective step length, $b$. Longrange interactions, such as the excluded-volume effect [20-22], disallowing conformations for which one section of an ideal chain would overlap with another, are important in determining the radius of gyration of the polymer. This radius is the average size of the polymer, and is an important parameter, particularly for the case in which the polymer is in solution. The interactions between solvent molecules and the polymer's constituent monomers determine whether the solvent plays a role in modifying the radius of gyration. A "good" solvent maximises the surface-to-volume ratio of the polymer and therefore swells it, while a "poor" solvent does the opposite by introducing a less favourable energy state to large polymer end-to-end vectors, thereby shrinking it. In this thesis much of the work carried out is either in the melt phase, or in polymer solutions in which molecular aggregation occurs, and the polymer end-to-end vector is more strongly determined by such effects than the excluded volume effect, so these long-range interactions are kept in mind, but are largely not influential in our analysis. 


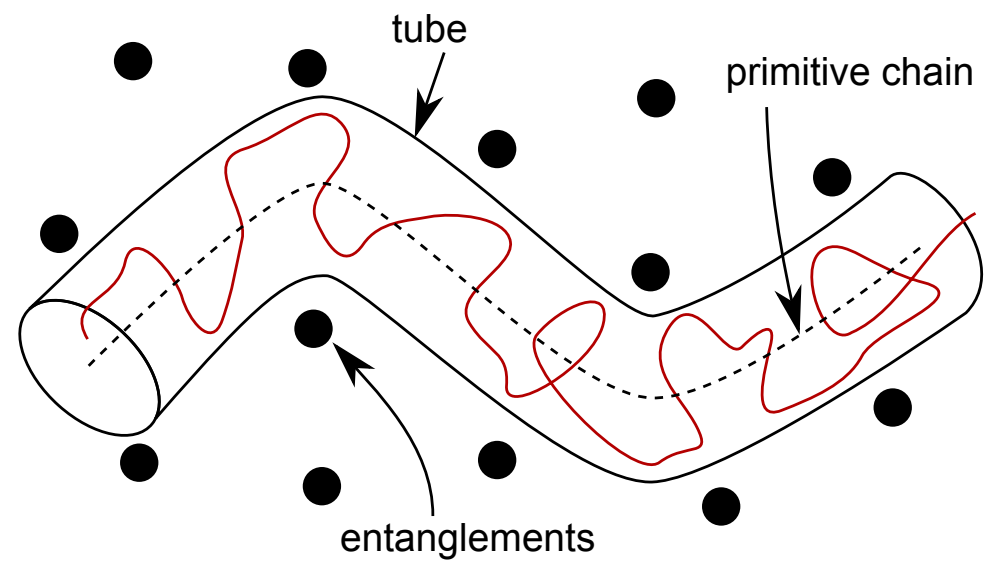

Figure 2.11: The polymer tube.

\subsubsection{Polymer melts and the tube model}

A material consisting entirely of free polymer molecules, and existing above its glass transition temperature is called a polymer melt. In such a fluid, a polymer molecule is more restricted in conformation that it otherwise might be, being confined to arrangements which trace paths around entanglement points (see fig. 2.11) generated by cohabitant polymers [23-26]. Edwards proposed [27] that the polymers doing the confining may be treated in a mean-field way, producing a tube in which a target polymer may freely diffuse due to thermal motions. The statistics of monomers in a given tube step of length, $a$, is then roughly Gaussian in the same way as we saw for the freely-jointed chain, however with the residual condition that at any given time, the local end-to-end vector is from one entanglement to the next. By expanding the view of the polymer in this way, or "zooming out", we may treat the step length, $a$, as a new length parameter in a random walk model, and statistically the distribution of overall end-to-end vectors would remain of the same form as if the polymer were free [28].

\subsubsection{Reptation and the tube model}

When polymers are packed densely enough, that is, the number of segments per unit volume $c^{*}$, the number of monomers $N$, and the radius of gyration (at infinite dilution) $R_{g}^{(0)}$, satisfy the relation 


$$
c * \approx \frac{N}{\left(R_{g}^{(0)}\right)^{3}}
$$

entanglement effects make themselves felt. In such a material of entangled polymers, normal diffusion statistics due to Brownian motion no longer apply to a molecule as a whole. The major mechanism for diffusion of a single polymer in a network of others is now one-dimensional and curvilinear in nature: it may diffuse along its own path. This process is known as reptation, a term coined by de Gennes [29] for its similarity to the propulsion mode of snakes. The tube in which a select polymer is located at some reference time will gradually disappear as the polymer diffuses back and forth, losing history of its location, beginning at the ends and eventually having the original tube disappear completely.

Abstracting the single, selected polymer away momentarily, the system can be modelled as a tube formed by restrictions, along with an idealised path along the centre of the tube, known as the primitive chain. As an idealised entity, the primitive chain maintains a constant length throughout its life, and is free to diffuse only along its own length, $L=\frac{N b^{2}}{a}$ with a diffusion coefficient $D_{c}$, which is provided by the Rouse model

$$
D_{c}=\frac{k_{B} T}{N \zeta}
$$

where $\zeta$ is a friction constant of polymer monomers. A schematic of this process is shown in fig. 2.12, showing the continual diminishment of the original tube. Once an end of the primitive chain has passed a given point, all the tube "outside" that point is lost irrecoverably. An analysis of this process, involving the probability that an end has not reached a given point given a diffusion coefficient yields [30] the probability that the central point of the tube still exists at all, as a function of time,

$$
\psi_{\text {tube }}(t)=\sum_{p \text { odd }} \frac{8}{\pi^{2} p^{2}} \exp \left(\frac{-p^{2} t}{\tau_{d}}\right)
$$

where $\tau_{d}$ is the characteristic time of the decay of the original tube. It is a highly important parameter in the description of polymer dynamics, known as the tube disengagement time, and represents the longest timescale for structural 

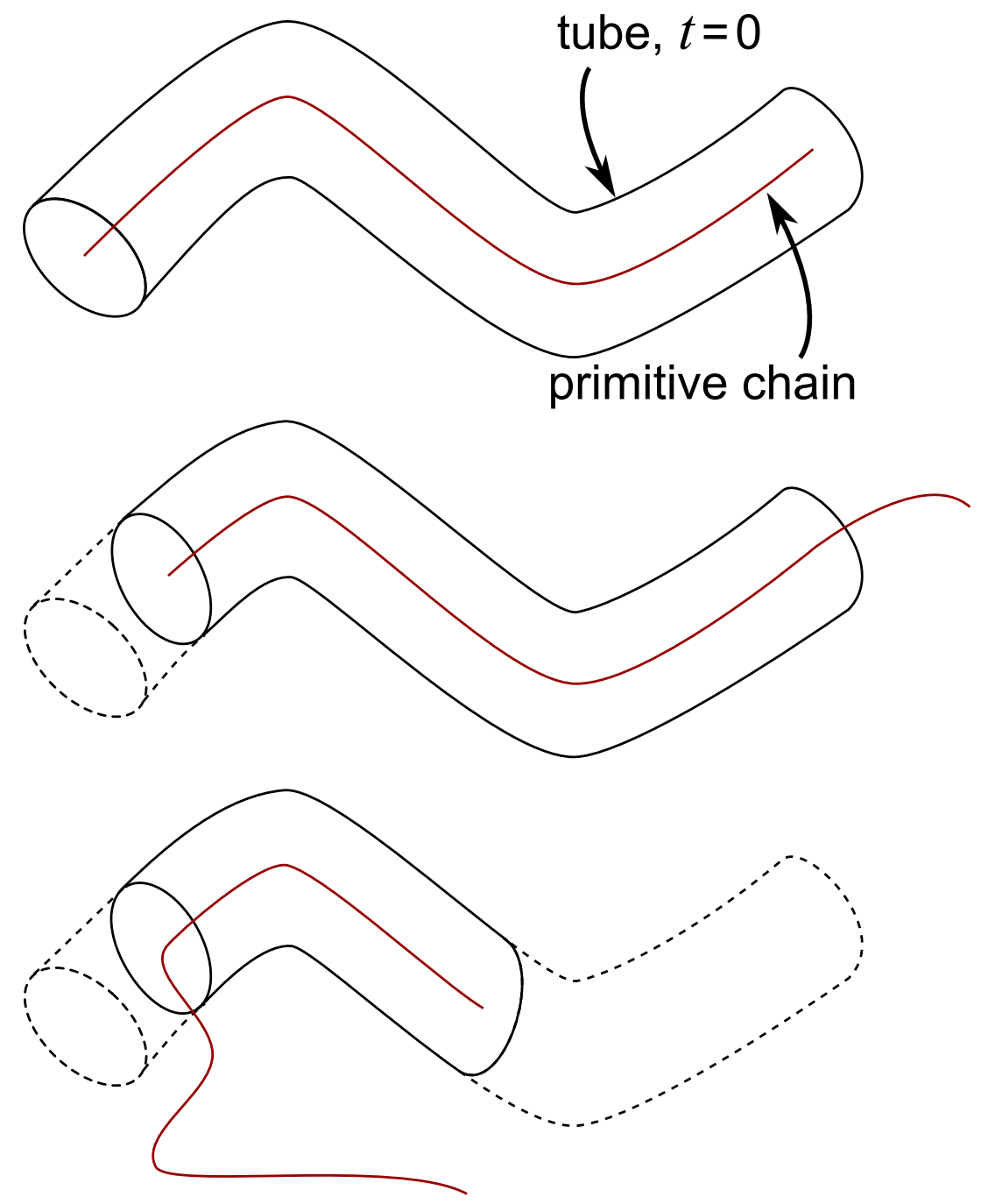

Figure 2.12: As time passes after a reference "snapshot", the tube in which a polymer resides is lost. Diffusion in one direction implies loss of tube at the other end, for if the polymer were to diffuse back, the end is free to take on any conformation, rather than be restricted to its original path. 
realignment in a polymer melt, and can be expressed in terms of the physical characteristics of the polymer as

$$
\tau_{d}=\frac{L^{2}}{D_{c} \pi^{2}}=\frac{1}{\pi^{2}} \frac{\zeta N^{3} b^{4}}{k_{B} T a^{2}}
$$

\subsubsection{Refinements to the Doi-Edwards model of polymer dynamics}

The Doi and Edwards model of polymer dynamics is a highly successful descriptor of the stresses and relaxation of stresses in an entangled network, and does so by using the various length- and time-scales (from Rouse motion up to 1D curvilinear diffusion/reptation) over which memory of the state of the sample is lost. There do exist factors that cause deviation from the simplest form of the Doi-Edwards model, though. One example is the convected constraint release (CCR) [31] phenomenon in which some account for the tube dissipation for one polymer may release an entanglement point for another polymer, and vice-versa. Contour length fluctuation [32] is another important correction to the simplest models, and is a result of the natural time-variability in some characteristic length-scales due to random thermal motions. Both these effects induce alterations in the dynamics of polymer melts, though being only considerable large as stress-relaxation mechanisms in certain regimes, will only be described as immediately necessary in later chapters.

\subsection{Surfactants and Worm-like Micelles}

\subsubsection{Molecular aggregation}

Molecules composed of sections which differ in their affinity for being close to water are known as amphiphilic. Those parts that shy away from water are called hydrophobic and those which seem attracted to water are called hydrophilic. In a material made up of water and a molecule which has both hydrophilic and hydrophobic parts, aggregation of the solute will occur due to minimisation of the free energy of the system. The hydrophobic portion will tend to be driven away from the water, and yet it remains physically attached to the hydrophilic. 


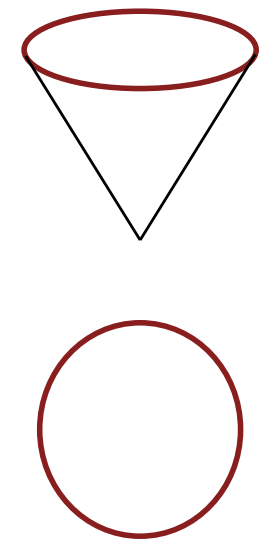

spherical micelles

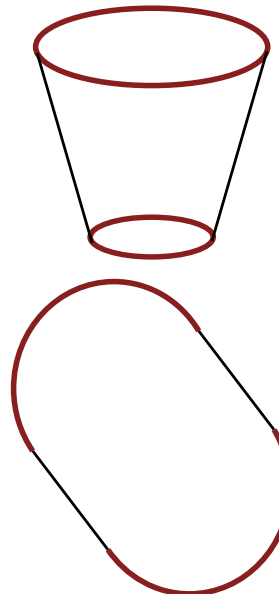

cylindrical micelles
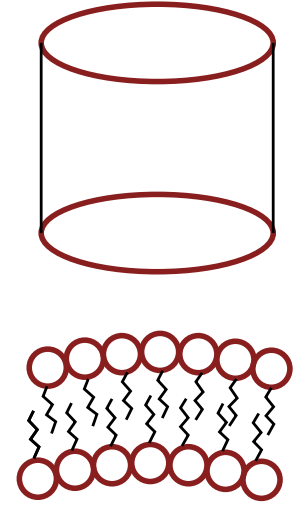

lamellae

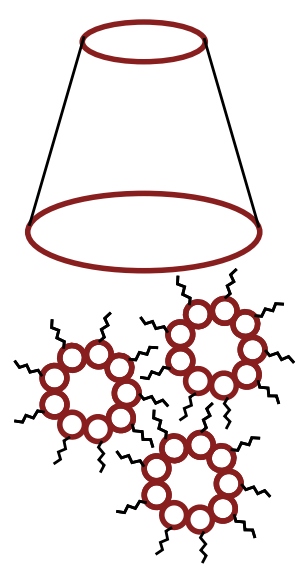

inverted micelles

Figure 2.13: An incomplete survey of typical structures of aggregate of surfactants in solution (see [1] for more details). Depending on the effective areas of head and tail groups (packing geometry shown at top), and the concentration of the surfactants in solution, a full spectrum of possible phases arise (lower figures). Worm-like micelles solutions may lie somewhere between short cylinder micelles and lamellae.

With the hydrophobic components having only affinity for themselves, it is not hard to imagine that under some circumstances small clusters of amphiphilic molecules will form in water. Other such structures are possible, and these aggregations are collectively known as micelles. Some micellar structures are shown in fig. 2.13 .

The tendency of an amphiphilic molecule as described above to aggregate into a larger structure is determined by the usual arguments between free energy and the entropy of mixing. Major determining factors are the concentration of molecules in the solvent, the system temperature, as well as - perhaps most importantly - the solvent-amphiphile electrochemistry [33]. The ionicity of the hydrophilic group plays the strongest role, and four general classes of system are outlined due to this ionicity: anionic, cationic, zwitterionic, or nonionic; where each refers to the charge on the surface-active portion of the molecule: negative, positive, both, or no charge at all. In many cases the hydrophobic parts of the molecule are alkyl chain hydrocarbons or similar, while the hydrophilic (and possibly ionic) ends may be made of carboxylic acid groups, or components 
such as sulphonates or amine salts.

No matter the system, micellar systems invariably do not exhibit molecular aggregation below a lower limit of concentration, known as the critical micelle concentration (cmc) [34]. Below the cmc, the free energy of micelle formation is insufficient to overcome entropic random mixing effects, while above it amphiphilic molecules can begin to lower the free energy sufficiently to form a spectrum of structures. At this limit many colligative properties change sharply due to aggregation: suddenly the effective number of "particles" is strongly reduced, and hence the cmc can be reasonably accurately determined from basic measurement of any one of these colligative properties as a function of concentration. In general, the $\mathrm{cmc}$ of nonionic micelles is lower than that of the ionic micelles, due to lower electrostatic repulsion between head groups.

In a similar vein, there exists a temperature below which micelles may not form at any concentration, due to lack of solubility. This temperature is known as the Krafft temperature [34], and at this point the solubility is in fact equal to the critical micelle concentration.

Once aggregation has been made possible through suitable conditions, the form of micellar structures is governed by free energy considerations, which are generally geometric in nature. The surface-to-volume ratio determines a surfactant parameter, $N_{s}=v /\left(l a_{0}\right)$, where $v$ and $l$ are the volume and length of the hydrophobic tail, respectively, and $a_{0}$ is a characteristic surface area of the hydrophilic head group. This parameter influences the curvature of the micelles, which may turn out to be spherical, cylindrical, planar, or inverted forms of these.

\subsubsection{Nonionic worm-like structure formation}

One additional structure which resemble long, flexible cylinders, or worms, are used by us as a model system in the soft condensed matter research in this thesis. They are most similar to the cylindrical structure shown in fig. 2.13, though are supposed to grow many times longer than their diameter. When such structures are formed, by the shielding of the hydrophobic components of the amphiphile by the hydrophilic parts (fig. ??), they are persistent structures which are reminiscent of linear polymers, though without the covalent bond- 


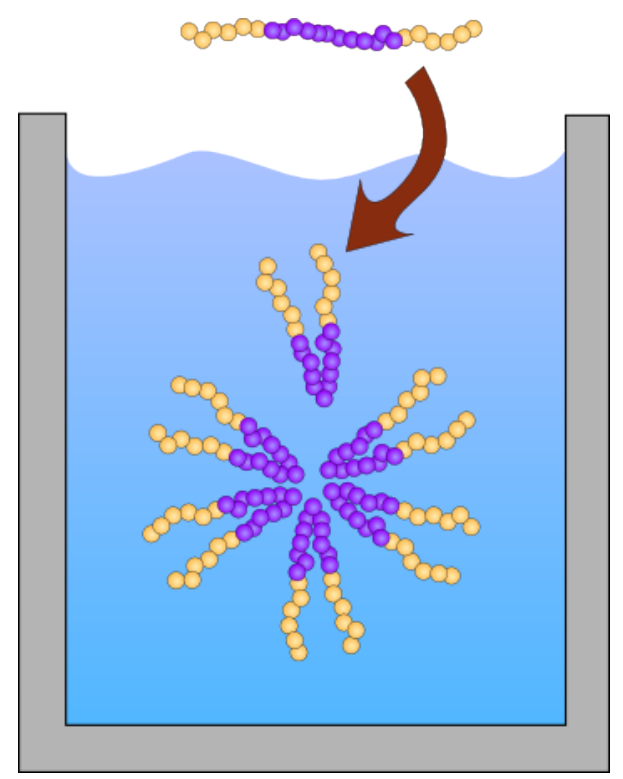

Figure 2.14: Simplified view of the way in which triblock copolymer segments demonstrate their dispositions towards the solvent, leading to micellar structures

ing seen in polymers, they are relatively easy to break apart and rejoin, these wormlike micelles are termed 'living polymers' [35].

In comparison to the qualitative properties of polymers, they can to first order be treated in certain concentrations by the tube model used previously for regular polymers, and hence the worms can be oriented under shear or extension. The main difference, however, is that the weaker intermolecular bonds and free energy minimisation that maintain the integrity of the structure are much weaker than the strong covalent bonds holding a polymer together - leading to a propensity for the worms to break up and recombine continuously, and a dissociation-recombination process has been described, largely by Cates [35, 36], which allows for a characteristic time over which a worm-like micelle may break apart and consequently recombine with other micelles in a Poisson renewal model of micellar kinetics. The effect of this is to introduce an alternative stress-relaxation mechanism which may be of shorter timescale than the tube disengagement time previously described for linear polymers, and this behaviour becomes apparent in the linear rheology of such fluids. Following the discussion of Maxwellian fluids of section 2.3.5, such short timescale effects are able to be quantitatively investigated at their correspondingly high character- 
istic frequencies, and are responsible for the deviations in the simple Maxwell fluid Cole-Cole diagram. Stress relaxation in polymer melts is associated with the dissipation of the polymer tube. With one-dimensional curvilinear diffusion being the dominant mode of stress relaxation, the ends of the primitive path have a large role to play in the rate of tube loss. As such, breakage and recombination of micelles generates additional opportunities for loss of time correlation of this mean field. The reptation-reaction model of Cates takes such timescales into account, and notes that the terminal relaxation time must be shorter than that determined by pure reptation alone. Bond interchange, in which two micelles collide in a fashion akin to the chromosomal crossover process in genetics (see figure 2.15), is responsible for a minor contribution to relaxation; however, reversible scission and end interchange are more dominant in accelerating stress relaxation. Selecting the shorter of the characteristic timescales for breakage and recombination and labelling it $\tau_{b r}$, the new terminal relaxation time can be written as a geometric average of the standard relaxation time due to curvilinear diffusion and the breakage time,

$$
\tau=\left(\tau_{\text {reptation }} \tau_{b r}\right)^{\frac{1}{2}}
$$

With such a wide range of processes occurring in micellar systems, it is not surprising that they respond to deformation both viscously and elastically, and can show highly non-linear behaviours, the most important of which for this research is shear banding.

In this research the majority of work undertaken is on the nonionic wormlike micelle system formed by the triblock copolymer P105 in solution, with the presence of the hydrophobic alcohol 1-phenylethanol providing a stabilising influence to the worms. Nonionic worm-like micelles do not require the presence of a counterion to generate the desired structure. The blocks of the copolymer are not in any way at the extremes of hydrophilicity or hydrophobicity, but the difference in solubility is enough to generate the worm-like micelle structure.

Once structures are seen to form under their corresponding required conditions, it is important to ask how the imposition of a shear field could possibly affect the state of the sample. In the most extreme case, a structureless homogeneous sample may obtain structure under shear, but more often it is the 

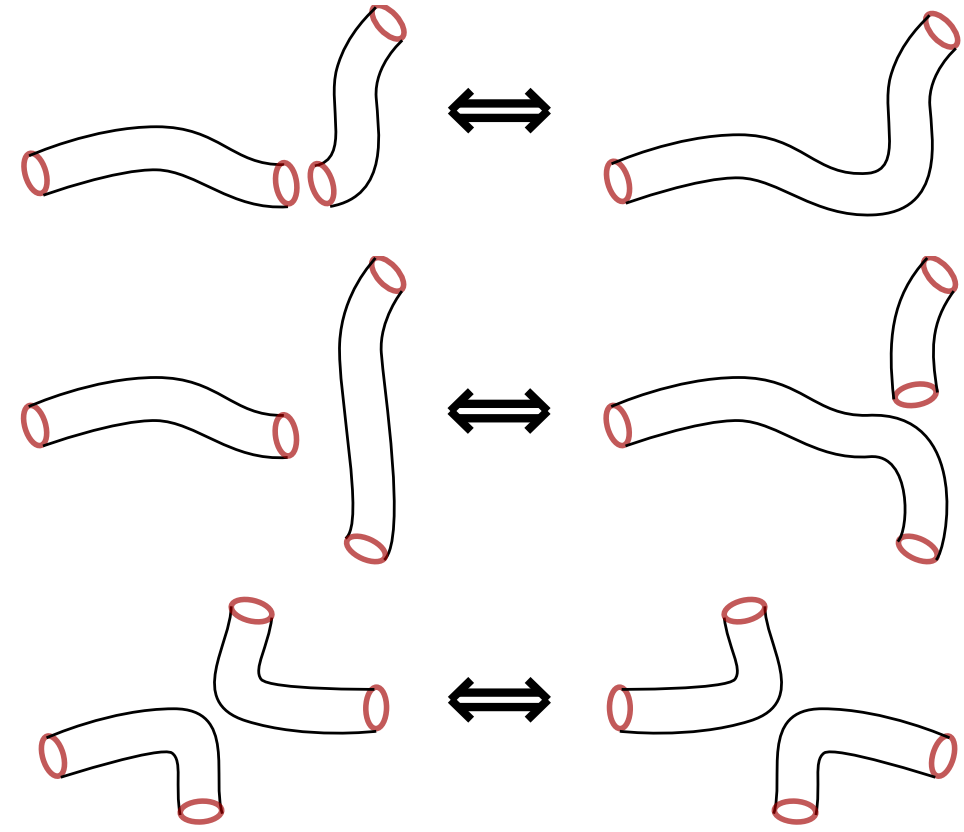

Figure 2.15: Three predominant mechanisms of micellar rearrangement (a) reversible scission (b) end interchange (c) bond interchange

transitions from exisiting structures that are of most relevance. In the case of lamellar phases, this may mean the transition to so-called onion phases [37, 38], or in the case of worm-like micelles which are known to have formed at rest, the isotropic-nematic transition is important. The level to which worm-like micelles will align under shear strain, and the possibility of an upper limit on the rate of strain which may be applied before micellar disintegration occurs, contribute to the non-linearity of the viscoelastic behaviour of such samples. Secondary effects such as the potential for micelles to grow longer under shear are not well explained, but mechanisms which diminish the relative rate of dissociation to combination seem plausible.

The geometric average of tube disengagement and breakage time of micelles, leading to an decrease in terminal relaxation time and non-monoexponential relaxation, is best shown up in the so-called Cole-Cole plot, which depicts the storage and loss modulus of the sample as a parametric curve with parameter $\omega$, the frequency of the linear rheology being performed. The expected behaviour for a perfect Maxwellian fluid is a semi-circle (see section 2.3.5), the frequency at which the curve for a given sample deviates from this behaviour is significant, 
and leads to knowledge of the ratio of breakage and disengagement times.

\subsubsection{Shear banding in worm-like micelles}

One of the most counter-intuitive phenomena displayed by worm-like micellar solutions under shear flow is that of shear-banding [1,39], in which two or more distinct spatial regions are seen to sustain different local strain rates, despite (nominally) maintaining a uniform stress - a variable viscosity.

Shear thinning is a well accepted and intuitively encountered phenomenon in which a lowered gradient in the shear-stress/strain function occurs with increasing shear rate. It is also a feature of many worm-like micelle solutions: one description of this phenomenon may be that more energy must be deposited into the system in order to cause the isotropic-to-nematic transition, aligning micelles, while subsequent shear deformation on aligned micelles is less forceintensive. In addition to shear thinning, however, it is been proposed that a non-monotonic constitutive relationship between stress and strain rate characterises the shear-banding phenomenon, as shown in fig 2.16. Materials developing a shear-banded flow behaviour within some range of imposed stresses demonstrate shear thinning behaviour in rheometric experiments, followed by a stress-plateau. The dissociation-recombination kinetics of micelles led Cates [36] to propose a constitutive equation incorporating birth- and death-rates of micelles; this was subsequently solved in non-linear rheological cases, demonstrating the stress/strain non-monotonicity previously described. While the addition of a declining stress regime in the tube model of Doi and Edwards [30] is thought to be the main instigator of such shear banding behaviour, alternative microscopic mechanisms have been suggested, for example, the local variation of some parameters of the solute such as concentration fluctuations or shear-induced structural changes [40]. Isotropic-to-nematic transitions induced by shear are apparent in worm-like micelle solutions, and the correlation of potential structural changes and regions of differing viscosity under a common stress is an important experimental area. Following Fischer and Callaghan [41], we will take on the investigation of the question of whether regions of differing birefringence (differing nematicity) are correlated with regions of differing viscosity. In the case that such correlation exists, it is interesting to ask the 


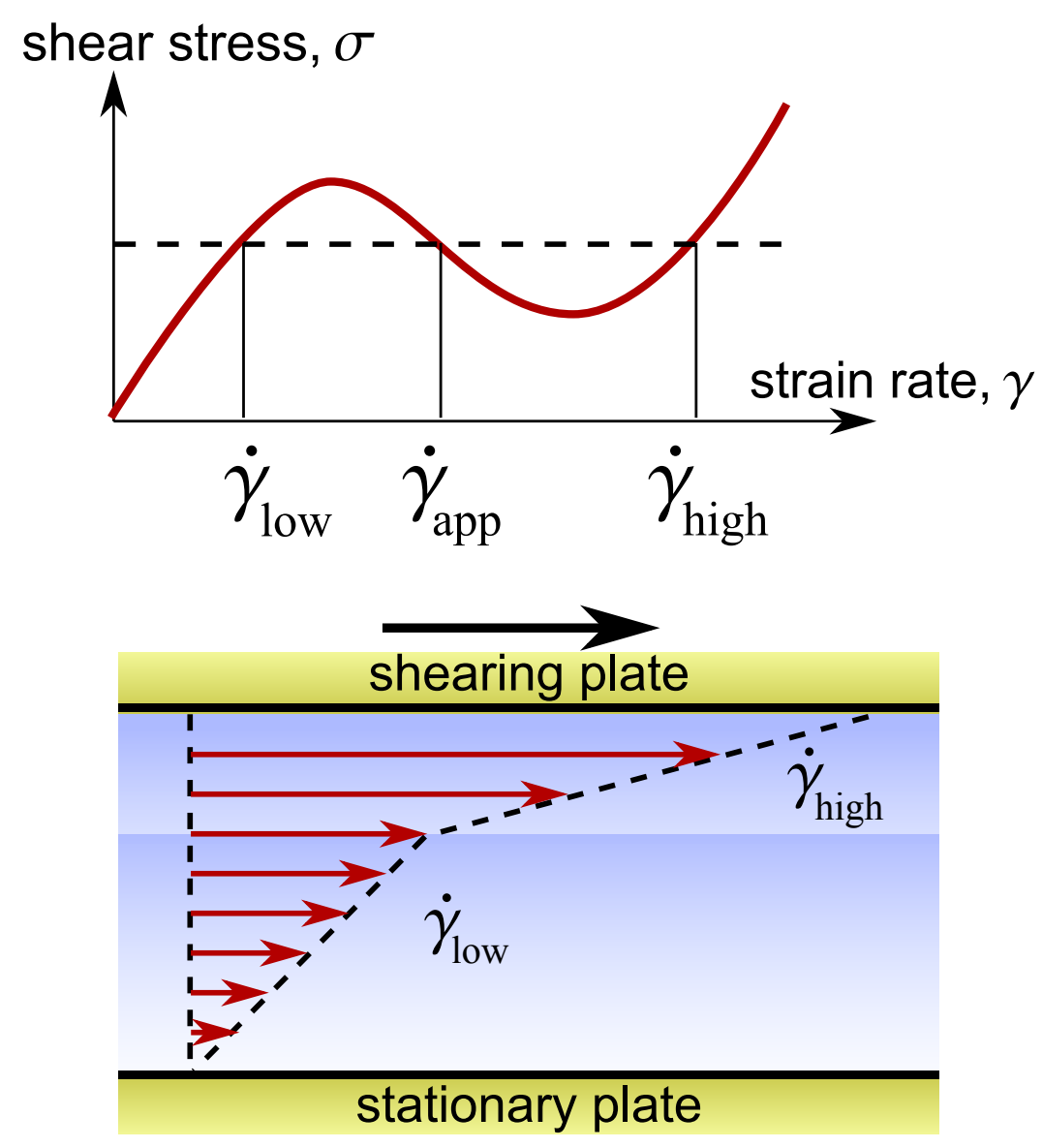

Figure 2.16: The non-monotonic nature of the flow curve is shown here. The negatively-sloped portions of the stress vs. strain function are inherently unstable, causing the fluid to shear-band. The bands take on the values of strain appropriate to the stress within the sample, as shown, and the band sizes satisfy a lever rule.

question: the phase of which ordered state corresponds to which of the highand low-shear-rate bands?

Theory and experiment continue to overlap in the area of shear-banding, whether in worm-like micellar solutions or not. In particular, shear bands have been experimentally shown to fluctuate across the gap of a rheometrical device, both in time and space, in both periodic and seemingly chaotic fashions. With such a wide variety of behaviours, worm-like micelles have become something of an ideal test-bed for investigating non-linear fluid mechanics [42], with their rich and diverse range of mechanical responses, despite being composed of relatively simple ingredients, which in many cases allow experiments that would 
possibly destroy the integrity of another sample. Trustworthy regeneration of initial conditions through self-assembly following a vigorous experiment is also a benefit. To add more drama to to the story of micellar materials, recently several groups have reported on materials which exhibit a stress plateau associated with shear-banded flow, followed by shear-thickening behaviour [43] and vorticity banding at even higher shear strain rates. 


\section{Bibliography}

[1] RG Larson. The Structure and Rheology of Complex Fluids. Oxford University Press, 1999.

[2] A Abragam. The Principles of Nuclear Magnetism. Clarendon Press, Oxford, 1961.

[3] B Blumich. NMR Imaging of Materials. Oxford University Press, New York, 2000.

[4] PT Callaghan. Rheo-NMR: Nuclear magnetic resonance and the rheology of complex fluids. Reports On Progress In Physics, 62(4):599-670, APR 1999.

[5] GG Fuller. Optical Rheometry of Complex Fluids. Oxford University Press, 1995.

[6] J Bear. Dynamics of Fluids in Porous Media. Dover, 1972.

[7] TE Faber. Fluid Dynamics for Physicists. Cambridge University Press, 1995.

[8] JD Ferry. Viscoelastic Properties of Polymers. Wiley, 1980.

[9] RB Bird, RC Armstrong, and O Hassager. Dynamics of Polymeric Liquids. John Wiley \& Sons, 1987.

[10] J Meissner. Rheometry of polymer melts. Ann. Rev. Fluid Mech., 17:45-64, 1985. 
[11] DM Binding, DM Jones, and K Walters. The shear and extensional flow properties of M1. Journal of Non-Newtonian Fluid Mechanics, 35:121-135, 1990.

[12] G. Fano. Contributo allo studio dei corpi filanti (contribution to the study of thread-forming materials). Archivio di Fisiologio, 5:365-370, 1908.

[13] FT Trouton. On the coefficient of viscous traction and its relation to that of viscosity. Proc. Roy. Soc. A, 77:426-440, 1906.

[14] R.G. Larson. Constitutive equations for polymer melts and solutions. Butterworths, page 364, 1988.

[15] CS Petrie. One hundred years of extensional flow. Journal of NonNewtonian Fluid Mechanics, 137(1-3):1-14, 2006.

[16] DF James, GM Chandler, and SJ Armour. A converging channel rheometer for the measurement of extensional viscosity. Journal of Non-Newtonian Fluid Mechanics, 35(2-3):421-443, 1990.

[17] DF James, GM Chandler, and SJ Armour. Measurement of the extensional viscosity of M1 in a converging channel rheometer. Journal of NonNewtonian Fluid Mechanics, 35(2-3):445-458, 1990.

[18] M Shirakashi, H Ito, and DF James. LVD measurement of the flow field in a constant-extensional-rate channel. Journal of Non-Newtonian Fluid Mechanics, 74(1-3):247-262, 1998.

[19] CS Petrie. Extensional viscosity: a critical discussion. Journal of NonNewtonian Fluid Mechanics, 137(1-3):15-23, 2006.

[20] PJ Flory. Journal of Chemical Physics, 17:223, 1949.

[21] PG de Gennes. Scaling Concepts in Polymer Physics. Cornell University Press, 1978.

[22] W Kuhn. Concerning the shape of thread shaped molecules in solution. Kolloid Z., 68:2-15, 1934. 
[23] M Doi and SF Edwards. J. Chem. Soc. Faraday Trans. II, 74:1789-1801, 1978.

[24] M Doi and SF Edwards. J. Chem. Soc. Faraday Trans. II, 74:1802-1817, 1978.

[25] M Doi and SF Edwards. J. Chem. Soc. Faraday Trans. II, 74:1818-1832, 1978.

[26] M Doi and SF Edwards. J. Chem. Soc. Faraday Trans. II, 75:38-54, 1978.

[27] SF Edwards. Proc. Phys. Soc., 91:513, 1967.

[28] ME Cates, TCB McLeish, and G Marrucci. The rheology of entangled polymers at very high shear rates. Europhysics Letters, 21:451, 1993.

[29] PG de Gennes. Reptation of a polymer chain in the presence of fixed obstacles. Journal of Chemical Physics, 55:572-577, 1971.

[30] M Doi and SF Edwards. The Theory of Polymer Dynamics. Oxford University Press, 1986.

[31] G Marrucci and G Ianniruberto. Die Makromolekulare Chemie. Macromolecular Symposia, 117:233-240, 1997.

[32] M Doi. Explanation for the 3.4 power law of viscosity of polymeric liquids on the basis of the tube model. Polym.Sci.Lett., 19:265-273, 1981.

[33] SJ Candau, A Khatory, F Lequeux, and F Kern. Rheological behaviour of wormlike micelles - effect of salt content. Journal de Physique II, 1994.

[34] D Dervichian and F Lachampt. The structure of soap solutions. Bulletin de la Societe Chimique de France, 12:189-206, 1945.

[35] ME Cates. Reptation of living polymers - dynamics of entangled polymers in the presence of reversible chain-scission reactions. Macromolecules, 20(9):2289-2296, 1987.

[36] ME Cates. Nonlinear viscoelasticity of wormlike micelles (and other reversibly breakable polymers). Journal of Physical Chemistry, 94(1):371375, 1990. 
[37] A Lutti and PT Callaghan. Undulations and fluctuations in a lamellar phase lyotropic liquid crystal and their suppression by weak shear flow. Physical Review E, 73(1):011710, 2006.

[38] Zilman and Granek. Undulation instability of lamellar phases under shear: A mechanism for onion formation? European Physical Journal B, 11:593608, 1999.

[39] S Fielding and PD Olmsted. Spatio-temporal oscillations and rheochaos in a simple model of shear banding. Physical Review Letters, 92(8):84502, 2004 .

[40] S Fielding and PD Olmsted. Flow phase diagrams for concentrationcoupled shear banding. European Physical Journal E, 11(1):65-83, 2003.

[41] E. Fischer and P.T. Callaghan. Shear banding and the isotropic-to-nematic transition in wormlike micelles. Physical Review E, 64(1):011501, 2001.

[42] H Rehage and H Hoffman. Viscoelastic surfactant solutions-model systems for rheological research. Molecular Physics, 74:933-973, 1991.

[43] ME Cates, DA Head, and A Ajdari. Rheological chaos in a scalar shearthickening model. Physical Review E, 66(2):025202, 2002. 


\section{Chapter 3}

\section{Magnetic Resonance Spectroscopy, Imaging and Velocimetry}

In 1945, two research groups headed by Bloch and Purcell [1, 2] discovered that nuclei in a magnetic field absorb and re-radiate radio-frequency electromagnetic radiation. The fact that the radiation frequency coincided with that described by the Larmor relationship between magnetic field strength and the nuclear spin precession frequency led the field to be called Nuclear Magnetic Resonance (NMR). Since that time a great number of innovations have contributed to this technique's applicability in modern research and technology as a molecular-level probe, most noteworthy of which is the use of magnetic field gradients to extend the capabilities of magnetic resonance out to spatial (and correspondingly velocity) resolution, a realisation which resulted in the Nobel Prize in Medicine being awarded to Lauterbur and Mansfield in 2003. Adding to these two major discoveries many incremental and quantitative enhancements, NMR has become the rich and varied field it is today, not least of all due to its usefulness in medicine and other non-invasive diagnostic applications.

In this thesis we use the subtle changes in the nuclear precessional frequency that arise due to the environment of the nucleus - determining the spectroscopic response of a magnetic resonance experiment - as well as the mapping of such spectroscopic data spatially - magnetic resonance imaging. As NMR is such a 
large field of research, a complete survey is not attempted here; instead, a short introduction is given with the intention of building the requisite tools for our investigations into the detection of various levels of ordering and flow in soft materials which arise due to macroscopic deformation. For further information and in-depth details on magnetic resonance in materials physics, the works of Abragam [3], Callaghan [4], Kimmich [5] and Blümich [6] are recommended references.

\subsection{Nuclear Spin}

Spin is an intrinsic property of elementary particles not known to have a classical analogue. While spin behaves as a contribution to the total angular momentum of a particle, it is known that particles have angular momentum about a point different from that expected due solely to orbital motion. In the formalism of quantum mechanics, every elementary particle has a spin angular momentum $I$, with a magnetic or azimuthal quantum number associated with it which may run from $-I$ to $I$ in the discrete spectrum $\{-I,-I+1,-I+2, \ldots, I-1, I\}$. The spin angular momentum of a particle also generates an intrinsic magnetic moment which is a vector collinear and proportional in magnitude to the observed spin component [7]

$$
\mu=\gamma \mathbf{S}
$$

where $\mu$ and $\mathbf{S}$ are the quantum mechanical operators for magnetic moment and spin respectively, and $\gamma$ is called the gyromagnetic ratio of the particle. We will be primarily interested in examining the properties of two nuclei: the single proton of hydrogen $\left({ }^{1} \mathrm{H}\right)$, with gyromagnetic ratio $26.75 \times 10^{7} \mathrm{~s}^{-1} \mathrm{~T}^{-1}$; and the bound proton-neutron nucleus of deuterium $\left({ }^{2} \mathrm{H}\right)$, with gyromagnetic ratio $4.11 \times 10^{7} \mathrm{~s}^{-1} \mathrm{~T}^{-1}$. The proton is spin- $\frac{1}{2}$ and has two observable values for the

magnetic quantum number $\left\{-\frac{1}{2}, \frac{1}{2}\right\}$, while the deuteron is spin- 1 and has three $\{-1,0,1\}$.

According to the matrix representation of quantum mechanics, these observable values for the spin angular momentum component are the eigenvalues of that operator, and the corresponding eigenvectors form a basis of the spin state 
space for a spin $i$.

\subsection{Spin and the Density Matrix}

Vast numbers of nuclear spins present in a real sample make it impossible to perform an experiment which keeps track of each spin as well as its observed orientation. For this reason a very useful mathematical device called a density matrix is used to describe the spin system to great accuracy.

In an ensemble of $N$ spins, the state of each spin is described by its spinor, $\left|\psi_{i}\right\rangle$, which for the spin-1/2 can be expressed as a linear combination of the standard basis with coefficients $\alpha$ and $\beta$

$$
\left|\psi_{i}\right\rangle=\left(\begin{array}{c}
\alpha_{i} \\
\beta_{i}
\end{array}\right)=\alpha_{i}\left(\begin{array}{l}
1 \\
0
\end{array}\right)+\beta_{i}\left(\begin{array}{l}
0 \\
1
\end{array}\right)
$$

and one can create a matrix operator

$$
\hat{\rho}=N^{-1}\left(\left|\psi_{1}\right\rangle\left\langle\psi_{1}|+| \psi_{2}\right\rangle\left\langle\psi_{2}\right|+\ldots\right)
$$

which, it can be seen, is an arrangement of averages of the spins' components

$$
\hat{\rho}=\left(\begin{array}{ll}
\overline{\alpha_{i} \alpha_{i}^{*}} & \overline{\alpha_{i} \beta_{i}^{*}} \\
\overline{\beta_{i} \alpha_{i}^{*}} & \overline{\beta_{i} \beta_{i}^{*}}
\end{array}\right)
$$

The diagonal components of this density operator can be identified as the average "up-ness" and "down-ness" of the spin ensemble, or the expected populations of the two observable spin orientations. The sum of these remains unity $\left(\left|\alpha_{i}\right|^{2}+\left|\beta_{i}\right|^{2}=1=\overline{\left|\alpha_{i}\right|^{2}}+\overline{\left|\beta_{i}\right|^{2}}\right)$ and the difference is in some way comparable to a spin excess. After a 'long' time left alone, the distribution of states of the spins in an ensemble of spins- $1 / 2$ (just the two eigenstates) will be governed by the Boltzmann statistics. As such in thermal equilibrium the value of the population difference will depend on the energy difference, $E$, between the two levels, and the temperature, such that in the high temperature approximation $E \ll k_{B} T$,

$$
\overline{\left|\alpha_{i}\right|^{2}}-\overline{\left|\beta_{i}\right|^{2}}=\frac{E}{k_{B} T}
$$


Elements of the density matrix not lying on the diagonal are called coherences. In thermal equilibrium, there will be zero transverse spin polarisation of the system. The $\alpha \beta^{*}$ coherence in the density matrix is directly correlated to the magnitude and phase of observed net transverse spin. We see later that it is only this component that is detected through induction in the conducting coil used in the magnetic resonance experiment. In this sense it is our job as experiment operators to use some recipe of manipulations to take the density matrix describing the initial state of the system and transform it by generating coherences that yield the information we would like.

\subsection{Spins in Magnetic Fields}

Should a particle with a magnetic moment such as that generated by the spin angular momentum be located within a magnetic field $\mathbf{B}_{0}$, it will experience the Zeeman interaction, and the system will have a potential energy

$$
H=-\mu \cdot \mathbf{B}_{0}
$$

associated with which are two observable energy levels

$$
E_{m}=-\gamma \hbar m_{I} B_{0}
$$

corresponding to the spin being 'up' (nuclear magnetic moment aligned with the $B_{0}$ field) or 'down' (magnetic field and nuclear magnetic moment anti-parallel). By absorbing and radiating radio-frequency photons of matching energy, the expectation of the magnetic moment can be modified: to change the expectation to zero would be to negate the Boltzmann population difference, and generate coherences in the density matrix.

The time-dependent Schrödinger equation governing the behaviour of the nucleus can be written

$$
\frac{d}{d t} \psi(t)=-\mathrm{i} \hat{H} \psi(t)
$$

and in the frame of the magnetic field the Hamiltonian operator is diagonal and proportional to the $I_{z}$ operator. Solving the Schrödinger equation is then 
relatively simple, and

$$
\psi(t)=\exp \left\{-\mathrm{i} \omega t I_{z}\right\} \psi(0)
$$

The exponentiation of the $I_{z}$ operator is equivalent to a rotation operator, and hence we have found that if the nuclear spin feels only a stationary magnetic field, it precesses about it. This constant rotation about the $z$-axis encourages us to move to an observation frame which rotates with the spin about the $z$-axis with frequency $\omega$. The spin appears stationary in this frame, and we can deduce that the effective magnetic field is zero. In general, our rotating frame may be characterised by frequency $\Omega$ and the effective field written

$$
B_{\text {eff }}=B_{0}+\frac{\Omega}{\gamma}
$$

\subsection{Relaxation}

\subsubsection{Phenomenological Relaxation}

Following excitation of the spins by the applied radio-frequency field, the density matrix may differ significantly from its thermal equilibrium state. Coherences (off-diagonal) elements will in general be non-zero, and the populations (diagonal) elements will not be determined by simple Boltzmann thermal statistics. With no thought as to the physical processes that drive these elements back to their thermal equilibrium values over the subsequent time, the so-called Bloch equations give simple insight into the semi-classical evolution of the magnetisation vector. In particular they separate two subclasses of relaxation - longitudinal and transverse relaxation, often called spin-lattice and spin-spin relaxation respectively. Longitudinal relaxation is best described by the rate at which the populations of the density matrix decay back to thermal equilibrium, the time constant associated with this decay is called $T_{1}$. There exist several related characteristic times depending on the frame of reference, but in the simplest case, the populations refer to the field-aligned $z$-component of the magnetisation, and as a guide, protons in aqueous solutions at room temperature often have $T_{1}$ values within an order of magnitude of $1 \mathrm{~s} . \quad M_{z}$ is governed by the 
following phenomenological differential equation as follows

$$
\frac{d M_{z}}{d t}=-\frac{\left(M_{z}-M_{0}\right)}{T_{1}}
$$

The other aspect of directly observable relaxation, at least for simple spin species $(I=1 / 2)$, is transverse relaxation, in which the elements belonging to the first superdiagonal of the density matrix (single quantum coherences) relax to zero. This is generally driven by spins dephasing, a process in which small additional precessional frequencies govern individual spin dynamics. The characteristic time taken for this to occur is known as $T_{2}$, and the transverse (NMR signal inducing) magnetisation follows the rule

$$
\frac{d M_{x, y}}{d t}=-\frac{M_{x, y}}{T_{2}}
$$

Combining these two relationships and a potential intrinsic inhomogeneity in the polarising magnetic field, one arrives at the Bloch equations [3], a triplet of differential equations governing $M_{x}, M_{y}$ and $M_{z}$. Transverse relaxation is of primary interest in this thesis, although the level to which we wish to describe the effect of alignment of polymers on the transverse relaxation time requires a greater level of understanding of relaxation mechanisms

\subsubsection{Dependence of transverse relaxation on dynamics}

In 1948, Bloembergen, Purcell and Pound (BPP) proposed a model of relaxation which relied on the tumbling motions of internal components of materials. Both longitudinal and tranverse relaxation are determined by fluctuations in the precessional frequency (nuclear Hamiltonian) felt by individual nuclei in accordance with their variable position and orientation with respect to each other. Molecular dynamics such as rotation and Brownian motion in general make for stochastic variations in spin couplings. Much like the Hamiltonian itself, there is a hierarchy of strengths of mechanisms by which relaxation can occur, and the quadrupolar interaction is certainly significant, if the sample permits; dipole interactions follow closely.

The temporal variation of the dipolar coupling between two nuclei within some proximity can be represented by a spectral density function, $J(\omega)$, which is 
an even, real and normalised function, and is derived from the auto-correlation function of a 'structure function' detailing the relative positions of neighbouring dipoles. Analysis of the relevant functions is highly sample-specific, and several regimes based on the amount of motional averaging possible on the NMR experimental timescale are treated independently. The BPP theory is particularly effective in homogeneous systems, though several other approximations exist that are more or less suitable for use in particular cases.

An important case for our work is that in which complete motional averaging is not achieved, and the Anderson-Weiss approach to the analysis of relaxation is appropriate. This method assumes that additional precessional frequencies (both positive and negative) cause the transverse dephasing of isochromats leading to relaxation of the normalised signal, $S(t)$,

$$
S(t)=\langle\exp (i \phi(t))\rangle
$$

where $\phi(t)$ is the time integral of additional phase acquired by random material influences relative to the central precessional frequency of the nuclear species

$$
\phi(t)=\int_{0}^{t} \omega\left(t^{\prime}\right) d t^{\prime}
$$

In evaluating the ensemble average of eqn. 3.13, it is then useful to take the cumulant expansion and retain only the second term, by virtue of other contributions being zero: those of odd order are automatically for our case, or not being consistent with an assumption of $\omega$ being distributed in a Gaussian way. Therefore,

$$
S(t)=\exp \left(-\frac{1}{2} \int_{0}^{t} d t^{\prime} \int_{0}^{t} d t^{\prime \prime}\left\langle\omega\left(t^{\prime}\right) \omega\left(t^{\prime \prime}\right)\right\rangle\right)
$$

which, in the case we represent and calculate a correlation function $C(t)=$ $\langle\omega(t) \omega(0)\rangle$, following Abragam [3] is

$$
S(t)=\exp \left(-\int_{0}^{t} d t^{\prime}\left(t-t^{\prime}\right) C\left(t^{\prime}\right)\right)
$$

This method is useful in polymeric systems for which we can calculate the 
correlation function depending on known system parameters, and in Chapter 4 , we see that the residual dipolar coupling generated by incomplete motional averaging of a nucleus bound to a polymer residing within the 'polymer tube' (for some residence time, or with a probability of return following diffusion) is a critical determinant of shear-dependent transverse relaxation in polymer melts.

\subsection{The nuclear spin Hamiltonian}

The nuclear spin Hamiltonian is overwhelmingly dominated by the Zeeman interaction between the nuclear magnetic moment and the strong $B_{0}$ field - but magnetic resonance spectroscopy and imaging also exploits the contribution of other interactions to the nuclear spin Hamiltonian.

The interaction between magnetic moments of nuclei, the chemical shift, and the scalar $J$-coupling are all examples of interactions generated by nuclear effects either between nuclei, or that are intrinsic to a nucleus, while the electric quadrupole coupling is a fascinating example of a nucleus of non-spherical charge distribution interacting with an electric field gradient like those existing in molecular orbitals.

These are the only interactions relevant to this work, and in order of strength in frequency units, the interaction hierarchy is as shown in fig.(3.1) (though it must be stressed that some of these quantities depend on the field strength, and the order presented here may not apply in other situations very much different than our experimental scenarios provide)

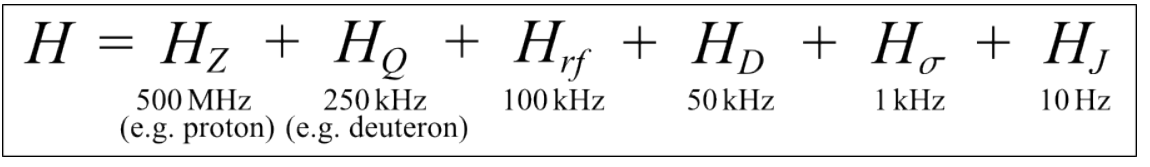

Figure 3.1: The hierarchy of the most common nuclear magnetic moment interaction strengths. In order, they are the Zeeman interaction (shown here for a proton in a modern high-field magnet) with the polarising field, the electric quadrupole interaction (for spins $I \geq 1$ ), the radio-frequency field, the dipole interaction, the chemical shift and the scalar $J$-coupling.

The strength of these various interactions is not, however, the only major player in the behaviour of spins once the collection of spins has been made 
to reside in a non-equilibrium condition through a change in populations and generation of coherences by a radio-frequency pulse. Molecular motion, and for the most-part irreversible molecular motion, leads to fluctuations of interactions between inter- or intramolecular spins, and it is these processes which form the majority of mechanisms for nuclear spin relaxation, which can be simplified in terms of the phenomenological approach already seen, or analysed in terms of the spectral density function. The spectral density appears as the Fourier transform of the autocorrelation function of the fluctuations in the spin interactions, and is a field of research unto itself. A simplified approach to the mechanism of proton transverse relaxation in polymer melts due to to dipolar interaction fluctuations shall be treated independently in Chapter 4 . In all other cases we shall simply refer to $T_{1}$ and $T_{2}$ as properties of a material or material component without further insight.

\subsubsection{The dipole interaction}

As objects with intrinsic magnetic moments, nuclei with spin influence (and are influenced by) other similar magnetic moments. While the effects these interactions have are much reduced from that of the overwhelming Zeeman interaction, they play an important part in the relaxation characteristics of spins and can affect the NMR lineshape heavily. The through-space dipoledipole interaction depends on their magnitudes, orientation and their relative positions within the sample. The expression for the interaction between two magnetic moments $\mu_{i}=\gamma_{i} \hbar I_{i}$ and $\mu_{j}=\gamma_{j} \hbar I_{j}$ is

$$
H_{D}=\frac{\mu_{0} \gamma_{i} \gamma_{j} \hbar}{4 \pi} \sum_{i, j} \frac{1}{r^{3}}\left[\mathbf{I} \cdot \mathbf{I}-\frac{3\left(\mathbf{I}_{i} \cdot \mathbf{r}_{i j}\right)\left(\mathbf{I}_{j} \cdot \mathbf{r}_{i j}\right)}{r_{i j}^{2}}\right]
$$

The small field due to one spin with dipole moment felt by another spin can be considered an inhomogeneity in the desirably homogeneous polarising magnetic field. However, fluctuations in $H_{D}$ due to molecular motion make for a different set of influences. Rapid isotropic motion, as in a small molecule in a liquid, leads to $H_{D}$ being averaged to zero, whereas static or slowly varying dipolar interactions broaden the NMR resonance and lead to more rapid $T_{2}$ relaxation. 


\subsubsection{The quadrupole interaction and the spin-1 deuteron}

A large part of experimental magnetic resonance, both in research and medical use, lies in the evolution and detection of ensemble nuclear paramagnetism of spin- $1 / 2$ protons. This is largely due to the proton's high gyromagnetic ratio as well as its overwhelming abundance in nature. However there are considerable benefits arising from the use of magnetic resonance of particles with spin greater than $1 / 2$. In particular, the deuteron's chemical similarity to the proton makes it a prime candidate for use as a proton substitute in experiment, despite minor isotopic effects such as reaction kinetics. While the deuteron has a gyromagnetic ratio only one-seventh of the magnitude of the proton's, its nuclear properties generate additional terms in the nuclear spin Hamiltonian that can produce information about the nuclear environment otherwise unattainable, such as the orientation of the carbon-deuteron bond within a molecule.

The quantisation of the spin-1 deuteron is such that if the angular momentum about a certain axis is measured, the potential outcomes are $-\hbar, 0$, and $\hbar$. It follows that the spinor representing an arbitrary spin is three-dimensional and can be written as

$$
\left|\psi_{i}\right\rangle=\left(\begin{array}{c}
\alpha_{i} \\
\beta_{i} \\
\gamma_{i}
\end{array}\right)=\alpha_{i}\left(\begin{array}{l}
1 \\
0 \\
0
\end{array}\right)+\beta_{i}\left(\begin{array}{l}
0 \\
1 \\
0
\end{array}\right)+\gamma_{i}\left(\begin{array}{l}
0 \\
0 \\
1
\end{array}\right)
$$

Accordingly, the density matrix for this three-dimensional spin-space is $3 \times 3$, and in thermal equilibrium is equal to

$$
\hat{\rho}=\left(\begin{array}{ccc}
\frac{1}{3}+B & 0 & 0 \\
0 & \frac{1}{3} & 0 \\
0 & 0 & \frac{1}{3}-B
\end{array}\right)
$$

where $B$ is a factor directly related to the Boltzmann thermal distribution of quantum states. Following this expression of the density matrix, we can note that the deuteron is the lightest nucleus to afford the possibility of multiple quantum coherences through single-spin interactions alone. Such coherences are not directly visible through electromotive induction in the r.f. coil, though multiple quantum filter techniques can be made to manipulate the density matrix 
to the point of transferring this information from the second superdiagonal to the first.

The non-spherical charge distribution located within the deuteron nucleus interacts with the surrounding electron clouds of the molecular orbital. The energy of the electric quadrupole interaction between the nucleus and orbital can be written

$$
H_{Q}=\frac{e Q V_{z z}}{4 I(2 I-1)}\left[3 I_{z}^{2}-I(I+1)\right]
$$

where $V_{z z}=\frac{\partial^{2} V}{\partial z^{2}}$ is the second derivative of the electric potential due to the molecular orbital in its natural frame of reference (along the bond direction, in general). $e$ is the unit charge on an electron, and $Q$ is the quadrupole moment of the nucleus. Asymmetry between the two other directions $V_{x x}$ and $V_{y y}$ is rare and may be taken to be negligible for carbon-deuteron bonds, the almost exclusive location of deuterons in this research.

As mentioned, the natural frame of reference in this research for the deuteron quadrupole interaction is along the carbon-deuteron bond, though assuming that such a bond is oriented at an angle $\theta$ to the main polarising field, the Hamiltonian contribution can be rewritten as the scaled quantity

$$
H_{Q}=\frac{e Q V_{z z}}{4 I(2 I-1)}\left[3 I_{z}^{2}-I(I+1)\left(\frac{3 \cos ^{2} \theta-1}{2}\right)\right]
$$

which in the case of the deuteron, of spin-1, produces a doublet spectrum, with splitting

$$
\Delta \nu=\left(\frac{3 e V_{z z} Q}{2 h}\right) \overline{P_{2}(\cos \theta)}
$$

In the case of fixed angle bonds this split signal can be averaged over for the bond angle distribution present within the sample. Molecules which are free to roam the entire sample in a short space of time, however, and undergo rapid anisotropic interactions with an aligning influence, should be effectively pre-averaged over the bond angle distribution, thereby reducing the splitting which is measured. The pre-averaging can be described by a scaling factor expressed through the spherical harmonic addition theorem, written here in terms of associated Legendre functions [8] 


$$
\begin{aligned}
& P_{n}(\cos \gamma)=P_{n}\left(\cos \theta_{1}\right) P_{n}\left(\cos \theta_{2}\right)+ \\
& 2 \sum_{m=1}^{n} \frac{(n-m) !}{(n+m) !} P_{n}^{m}\left(\cos \theta_{1}\right) P_{n}^{m}\left(\cos \theta_{2}\right) \cos m\left(\varphi_{1}-\varphi_{2}\right)
\end{aligned}
$$

which for our purposes allows for the intermixing of two separate order parameters involving a degree of order within an environment aligned along $\left(\theta_{1}, \varphi_{1}\right)$ and that environment itself having a degree of orientation along $\left(\theta_{2}, \varphi_{2}\right)$, separated by an angle $\gamma$. In the case of fluctuating azimuthal angles, and with the appropriate averaging and angles relevant to whichever director we are referring to, this leads to

$$
P_{2}(\cos \theta)=\left\langle P_{2}\left(\cos \Theta_{\mathbf{u}}\right)\right\rangle P_{2}(\cos \Theta)
$$

where $\theta$ is the angle a particular segment of directed media makes with the polarising field, and $\Theta_{\mathbf{u}}$ is the angle between the molecular bond axis and the director of the domain causing the alignment. $\Theta$ is the angle between the domain director and $B_{0}$. For this reason, there arises a difference in the information gained in the spectroscopy of aligned materials. If the C-D bond is physically attached to a polymer which is aligned, any pre-averaging will arise from local segmental motion, with respect to one alignment axis. On the other hand, If the C-D bond is part of a small probe molecule free to take a sampling of alignment within the material (which forms the basis of the use of deuteron spectroscopy in this thesis), then pre-averaging involves not only the segmental motion but also the tumbling of the probe molecule, and therefore the splitting is much reduced.

\subsubsection{The chemical shift interaction}

Upon being placed in a strong polarising magnetic field, currents in the electron clouds of a molecule are spontaneously induced. Molecular orbitals are slightly modified so as to change the local field and hence the Larmor frequency at which the enclosed nuclei precess, and this change in Larmor frequency is dependent on the chemical environment. This shift is know as the chemical shift, and while 
it is on the whole weaker than either of the dipolar or quadrupolar Hamiltonian contributions, forms the basis of NMR spectroscopy as a tool for elucidating chemical structure.

In our work, the chemical shift is used in conjuction with diffusometry in order to identify peaks in the NMR spectrum and associate distinct nuclear species with different diffusion coefficients in an experiment known as the DOSY.

\subsection{Signal detection}

If the bulk magnetisation has a component in the $x y$-plane perpendicular to the $B_{0}$ field, electrons in a wire coil sitting around a sample volume will experience an electromotive force due to its precession. The alternating current generated will be the Fourier sum of all the Larmor frequencies being exhibited by the nuclei within the sample. This sum is referred to as the NMR signal, which is then mixed with an oscillatory function close to the Larmor frequency, a process called heterodyne mixing, the result of which is an audio-frequency signal.

In the practice of Fourier transform NMR, this process is duplicated by another receiver which mixes the signal with an oscillating function of identical frequency, though $90^{\circ}$ out of phase. By this route, values for the transverse magnetisation can be obtained along two directions in the $x y$-plane, and the magnetisation is said to have been detected in quadrature [9].

For the simplest NMR experiment, that of the spectroscopy of water located in a suitably homogeneous polarising magnetic field, all protons within the sample will nominally precess at the same rate $\omega$, and their magnetisation as seen by the receiving coil may be written

$$
M(\cos \omega t+i \sin \omega t)=M \exp (i w t)
$$

In more complicated examples - and in general all experiments of interest - this expression will be converted into something multiexponential due to the contribution of different spins precessing at different rates. The form of the signal resulting from a single r.f. field pulse, which is characteristically enveloped by a decay curve due to spin-spin relaxation is called the free induction decay. Many other more complicated scenarios can be envisioned, for example signals 


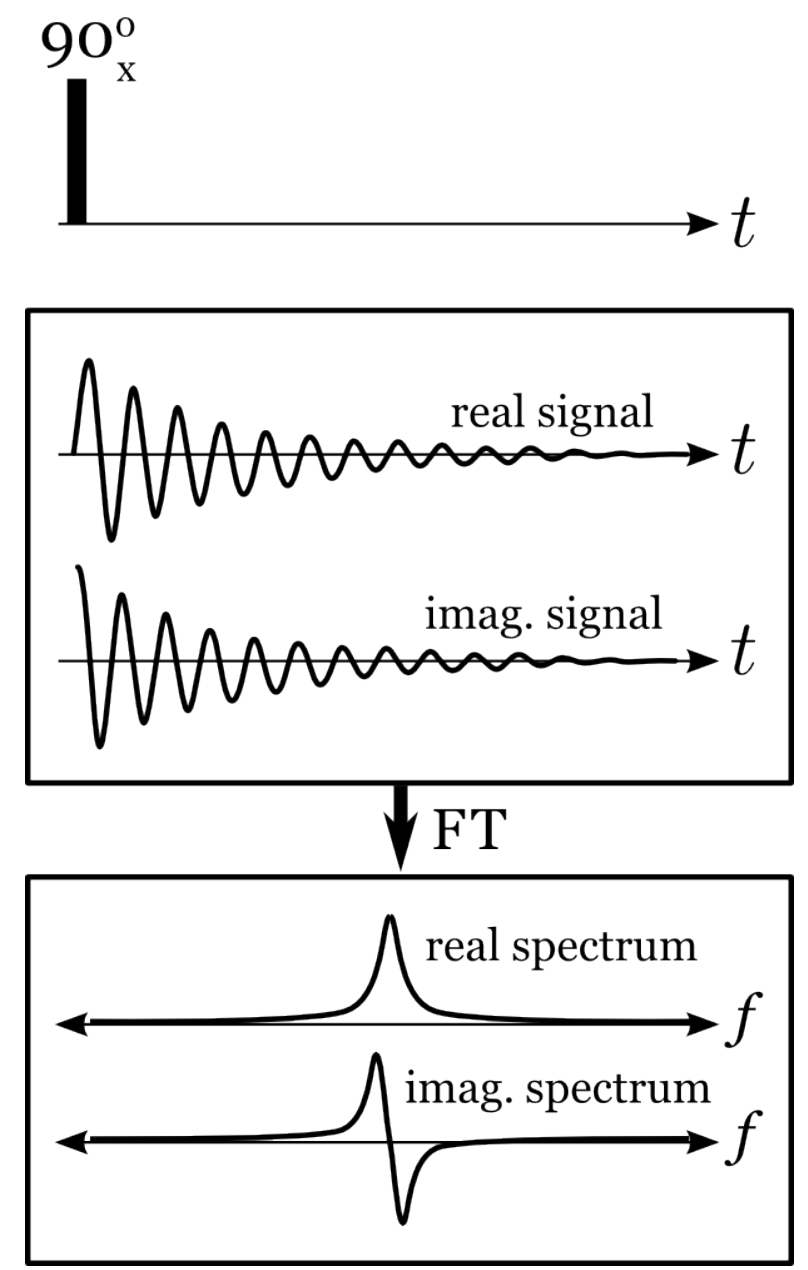

Figure 3.2: Following a $90^{\circ}$ radio-frequency pulse, transverse magnetisation is generated, the $x$ and $y$ projections of which may be considered the real and imaginary parts of a complex signal. Fourier transformation of such a signal yields its (complex) frequency decomposition.

can be detected by turning on the receiver at the appropriate time, the classic example of which is detecting the Hahn spin echo generated at a time $2 \tau$ after a $90^{\circ}$ pulse by applying a $180^{\circ}$ pulse at time $\tau$. This is the basis of pulse program design.

\subsection{Signal averaging and phase cycling}

In any experiment, the signal-to-noise ratio is important. For practical magnetic resonance experiment at the fields and ambient conditions we are likely 
to encounter, the spin excess generated due to Boltzmann statistics is a very small fraction of the total number of spins. It is therefore beneficial to repeat an NMR experiment and co-add the signals obtained.

Many sources of noise may encroach on an experiment, but due to their random nature, when $N$ successive signals are combined, they add with random phase, languishing where the desired signal grows proportionally to $N$. Over many experiments, the signal-to-noise ratio increases at a rate proportional to $\sqrt{N}$. Unfortunately consecutive experiments are not, in general, able to be stitched together contiguously without delay. The evolution of the density matrix is heavily dependent on the initial conditions; without being able to ensure what the initial conditions of the sample are, analysis is made considerably more difficult. By waiting an appropriate time to allow all sub-systems within the sample to relax (generally we need wait several spin-lattice relaxation times) we have re-attained thermal equilibrium and are free to execute the experiment again and co-add the signals.

Given the necessity of repeating acquisitions to obtain attractive and compelling data, there is no loss involved in employing the technique of "phase cycling', although phase cycling is indeed important even should the signal be strong and signal averaging is not required.

To demonstrate the use of phase cycling, consider the result of an r.f. pulse designed to tip the bulk magnetisation from the $B_{0}$ direction down into the plane perpendicular to it. If the experimenter chooses a pulse time slightly mismatched to that required, the signal generated will not be exactly that required. While in the case of a simple free induction decay method this results only in a slight loss of signal, in more complicated experiments involving many pulses it becomes vital [10].

By choosing a different axis lying in the $x y$-plane about which to rotate the magnetisation by way of r.f. field application, small errors in pulse time, errors due to phase and amplitude mismatch in receivers, as well as undesirable baseline artefacts can be removed from the NMR experiment. Many different phase cycling techniques exist, they shall be outlined relative to the specific pulse programs as required. 


\subsection{Magnetic Resonance Imaging}

Though the quality of a finely tuned, homogeneous magnetic field has been shown to be of high priority in magnetic resonance hardware design and implementation, a deliberate and systematic field inhomogeneity was proposed by Lauterbur and Mansfield $[11,12]$ to obtain spatial resolution. This technique has turned magnetic resonance imaging into a highly worthwhile pursuit, especially in medicine where MRI magnets have become part of the furniture of every hospital.

By applying a linear magnetic field gradient to the sample volume, nuclear precession frequency correspondingly varies spatially

$$
\omega(\mathbf{r})=\gamma B_{0}+\gamma \mathbf{G} \cdot \mathbf{r}
$$

where $\mathbf{G}$ is the field gradient

$$
\mathbf{G}=\nabla B_{0}=\frac{\partial B_{0}}{\partial x} \hat{\mathbf{i}}+\frac{\partial B_{0}}{\partial y} \hat{\mathbf{j}}+\frac{\partial B_{0}}{\partial z} \hat{\mathbf{k}}
$$

The NMR signal, $d S$, arising from a small sample volume, $d V$, is proportional to the spin density at that point as well as being dependent on the local Larmor frequency.

$$
d S \propto \rho(r) d V \exp (i \omega(r) t)=\rho(r) d V \exp \left[i\left(\gamma B_{0}+\gamma \mathbf{G} \cdot \mathbf{r}\right)\right] t
$$

Ignoring the $\gamma B_{0}$ term, due to an arbitrary reference phase being able to be chosen in practice, the signal can therefore be expressed as the integral

$$
S=\int_{V} \rho(\mathbf{r}) \exp [i \gamma \mathbf{G} \cdot \mathbf{r} t] d \mathbf{r}
$$

and hence by the substitution of the wave vector $\mathbf{k}=\gamma \mathbf{G} t / 2 \pi$, we arrive at a Fourier relationship between the two conjugate spaces $\mathbf{k}$ and $\mathbf{r}$. It is apparent we can obtain an image of the nuclear spin distribution by acquiring the NMR signal while applying a magnetic field gradient to the sample, varying the field gradient adequately so as to cover $k$-space to a desirable degree. 


\subsubsection{The Hahn spin echo}

While spectroscopy can gainfully be employed by the application of a single $90^{\circ}$ radio-frequency pulse, the advantages in applying a $180^{\circ}$ pulse at a time $\tau$ after the generation of transverse magnetisation by the $90^{\circ}$ r.f. pulse are so numerous that echo-based experiments are ubiqitous. Inhomogeneities both in composition of the sample and of the $B_{0}$ field generate additional precession frequencies, the result of which is a dephasing of the ensemble NMR signal. This de-phasing can be greatly reduced by applying the $180^{\circ}$ pulse about an axis lying in the $x y$-plane (see fig. 3.3), such that any spin sub-species precessing faster than the average Larmor frequency, and has thus gained an extra phase $\phi(\tau)$ now lags the central frequency by an angle $\phi(\tau)$ and so at time $2 \tau$ it once again in phase with the central frequency species, at which time the receiver is activated, and the echo is acquired [13].

The underlying assumption with this rephasing is that the additional frequencies imparted to a sub-species remain constant throughout the experiment, a condition applicable to single-spin interactions such as chemical shift effects and (in the absence of long-range motion) inhomogeneous broadening caused by macroscopic magnetic field variations. Conversely, perfect rephasing of signal is not achievable as there is always a contribution from homogeneous broadening: a process which is not limited to, but characterised well by, the example of non-reversible motion (like diffusion) carrying a molecule to a region of slightly differing magnetic field over the course of the experiment.

\subsubsection{Volume selection with field gradients}

Following the imposition of a known systematic field inhomogeneity, a positiondependent field strength and hence position-dependent precession frequency prevails. By using a shaped pulse (slice selective or soft pulse) of radio-frequency radiation, it is possible to screen the NMR signal such that the only contributions originate from a select volume within the sample.

This can be achieved by only irradiating the sample with the radio-frequencies corresponding to the precession frequencies in that volume. In the simplest case of volume selection, a gradient in magnetic field strength is imposed on the sam-

ple in a polarising field. The envelope of radio-frequency radiation is known as 

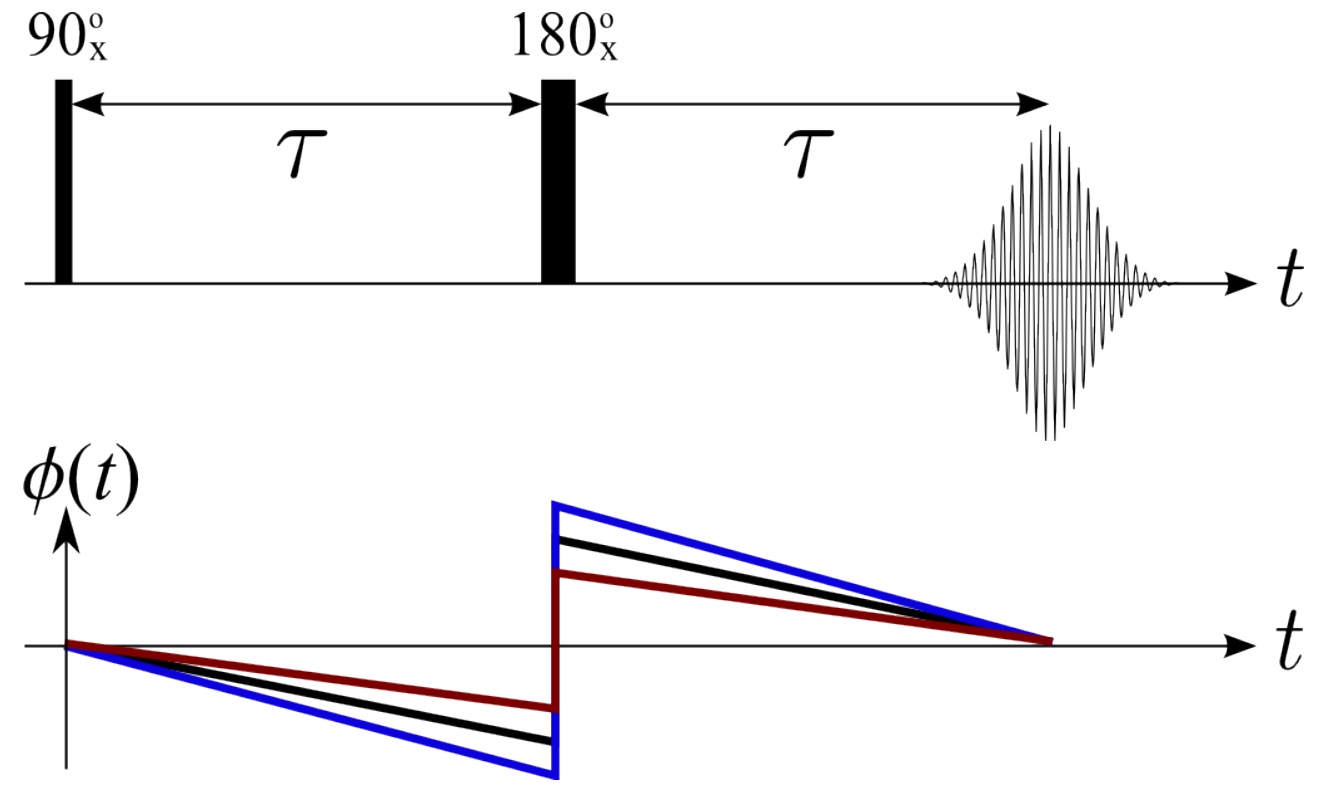

Figure 3.3: The Hahn echo pulse program, consisting of $90^{\circ}$ and $180^{\circ}$ pulses. Below is shown the result of refocussing the effects of inhomogeneous broadening. The blue line shows the phase of a spin precessing slightly faster than the average, while the red line corresponds to one precessing slower. Because these differences are due to reversible effects, the slope $d \phi / d t$ is the same before and after the $180^{\circ}$ pulse, leading to refocussing at time $2 \tau$. 

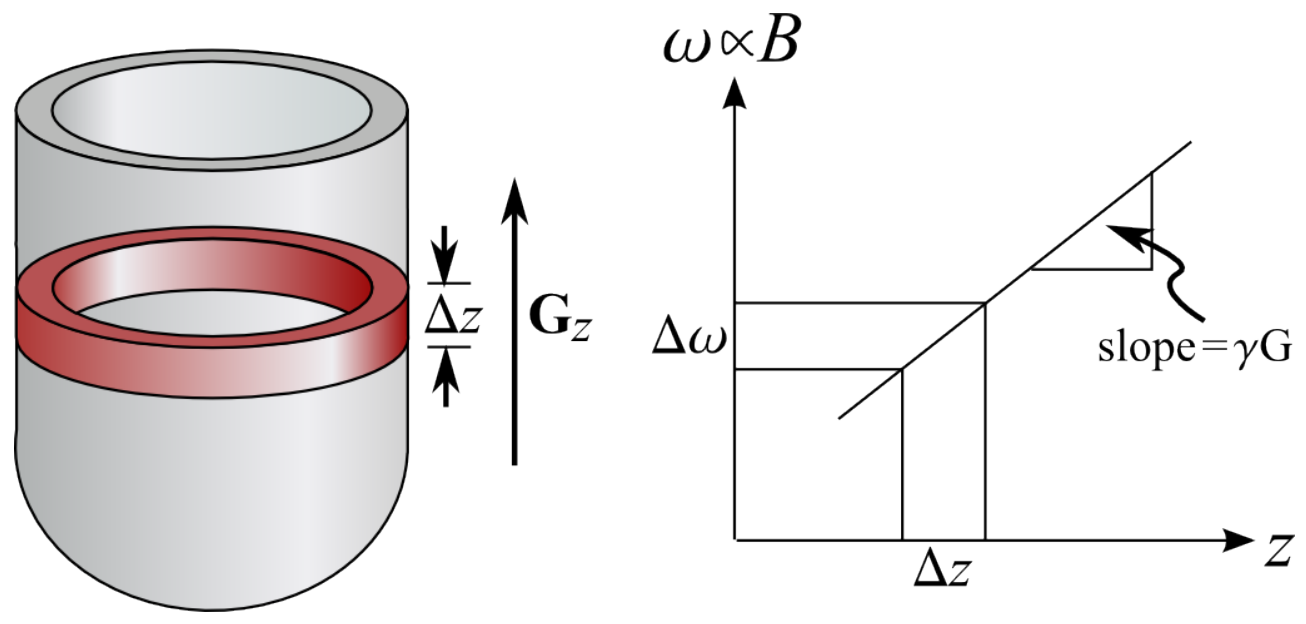

Figure 3.4: The restriction of signal to a selected volume shown schematically

the soft pulse shape, and is the Fourier inverse of the distribution of frequencies being excited. Typically in our research an envelope of $(\sin t) / t$ (or similar) envelope is used, truncated to a small number of nodes, which has as Fourier inverse a standard hat function with inevitable small oscillations due to the truncation. This characteristic "on or off" form of the hat function implies that only spins precessing within this bandwidth (and hence localised spatial region in case of applied gradient) are excited. We have gained a way of effectively excluding the effects of spins outside this region to a high degree. The relationship determining the "slice thickness" and gradient strength is $\Delta \omega=\gamma G \Delta z$, the schematic effects of simple implementation of this principle is shown in fig.3.4

\subsubsection{The projection profile}

As an example of a simple NMR experiment incorporating several of the basic components described previously, and required for rheo-NMR, the projection profile pulse program is outlined; its pulse program is shown schematically in fig.3.5. By incorporating a $180^{\circ}$ soft pulse of r.f. radiation, at the same time as a magnetic field gradient is induced across the sample, the acquired signal is preferentially received only from a restricted quasi-two-dimensional volume (i.e. the two-dimensional region still retains "thickness", yet each particular point within the volume contributes to the experiment as though it can be located with only two co-ordinates). As we have seen the thickness of the slice may be 


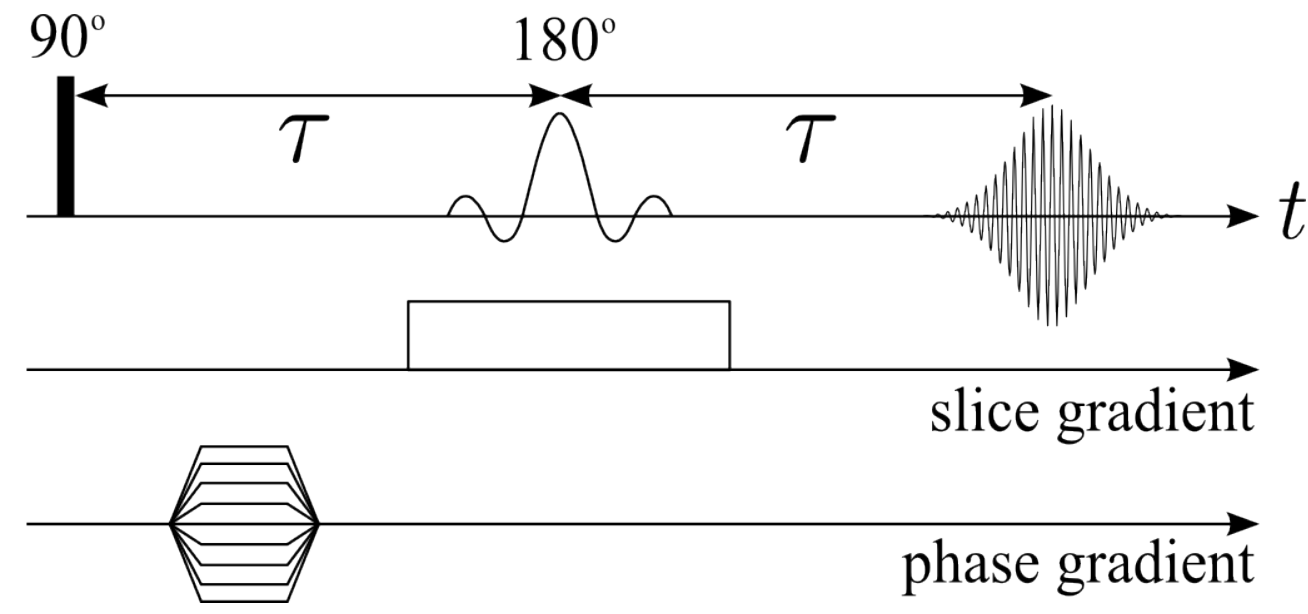

Figure 3.5: The projection profile imaging pulse program. The soft $180^{\circ}$ pulse is applied during a slice gradient to perform refocussing on a selected volume, while a phase gradient is applied in steps (incremented after each scan) to traverse a single line of $k$-space. In fact a 1D image could be obtained through the use of a gradient applied during acquisition (read gradient), however this sequence retains spectroscopic information in addition to spatial information and is used in radial spectroscopic imaging experiments.

written $\Delta \omega=\gamma G \Delta z$, where $\Delta \omega$ is the r.f. pulse bandwidth, and the gradient is made to lie in the $z$ direction.

In the case of the projection profile, we have been given time through the use of the echo to apply a gradient pulse in the absence of r.f.radiation so as to impart the phase required to traverse $k$-space along a line in incremental steps. In the instance that only one line of $k$-space (passing through the origin) is acquired, the Fourier transform of the projection profile has been obtained. It is effectively an integral of spin density in one direction as shown in fig.3.6.

\subsubsection{Radial spectroscopic imaging}

A particularly common experiment in magnetic resonance imaging is that of traversing $k$-space in two dimensions, that is, imaging the spin density in a plane following a slice selection procedure in one direction. Most objects imaged in magnets around the world are not cylindrically symmetric within this plane, and hence $k$-space must be fully explored in both dimensions to fully map this image. 


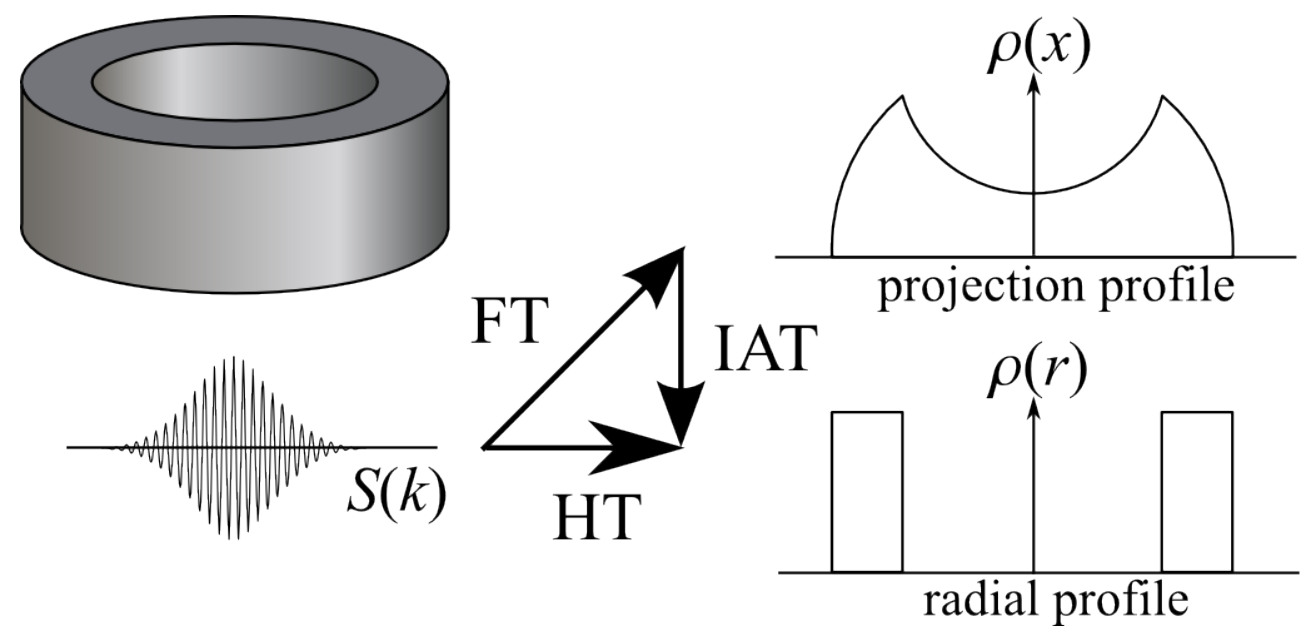

Figure 3.6: An ideal cylindrical Couette geometry, along with the result of performing the projection profile experiment described previously. The triangular relationship between Fourier, Hankel and Abel transformations is shown.

A technique known to the signal processing community, and in particular analysts of astronomical data, is the expression of the two-dimensional Fourier transformation of a function of $r=x^{2}+y^{2}$ only in polar co-ordinates, which has the result of producing the Hankel transformation. It is an integral transformation similar to the Fourier in which the zeroth-order Bessel function replaces the exponential function as kernel

$$
\rho(r)=\mathcal{H}[S(k)]=2 \pi \int_{0}^{\infty} S(k) J_{0}(k r) k d k
$$

This transformation forms a triangle along with those of Fourier and Inverse Abel, in which it can be shown that the result of the Fourier transformation followed by Inverse Abel transformation is equivalent to the Hankel transform of the original function (fig. 3.6). In practice, a two-step transformation method is used as opposed to the Hankel alone, for the main purpose of providing an intervening step in which signal processing can occur in a domain which is well known - the Fourier domain. That the Hankel transformation corresponds with the 2D FT is heavily reliant on centring of the data in both conjugate spaces. As such, being able to shift an image in Fourier space and remove baseline artefacts is necessary before inverting the Fourier transformation and finally applying the Hankel transformation is an economic process [14]. 
By using this technique, we reduce the number of variables over which the signal varies by one, turning the two-dimensional imaging effort into a onedimensional experiment, or a three-dimensional undertaking - whether this is a 3D image, or 1D spectroscopic information resolved over a 2D region - into a two-dimensional experiment. This can obviously lead to huge conservations in the duration of an experiment, as well an increasing signal per voxel: the signal received originates over a whole extra dimension! These experimental benefits will be of most use to us in the detection of deuteron NMR spectra, where signals are weak due to gyromagnetic ratio reduction and sparseness of probe molecules in our samples relative to protonated experiments.

\subsubsection{Diffusometry}

Magnetic field gradients may also be used to obtain diffusion information through magnetic resonance. In particular the use of pulsed-gradient spin-echo (PGSE) techniques allow measurement of signal attenuation associated with the displacement of an ensemble of sample nuclei over a well-defined time period. With this technique a pair of magnetic field gradient pulses surrounding a simple Hahn echo $180^{\circ}$ r.f. pulse causes the phase acquired by a spin ensemble prior to the pulse to be negated during the field gradient pulse afterwards. Should the ensemble experience diffusive motion in the interim, dephasing will occur resulting in an attneuated NMR signal.

Three parameters characterise a PGSE pair of field gradient pulses: their individual duration $\delta$, amplitude $g$ and the time (the observation time) between them $\Delta$, see fig.3.7. The degree of signal attenuation directly correlates with the r.m.s. distance travelled by the molecules. The residual phase acquired by the spins in a time $t$ can be denoted $\phi(t)$ and the attenuation of the normalised echo signal will then be the average over the whole spin ensemble, given by

$$
E(t)=\langle\exp [i \phi(t)]\rangle
$$

which in the case of ordinary unrestricted diffusion is equal to the StejskalTanner equation [15] 


$$
E(g, \delta, \Delta)=\exp \left\{-\gamma^{2} g^{2} D \delta^{3}\left(\frac{\Delta}{\delta}-\frac{1}{3}\right)\right\}
$$

By fitting such attenuation factor data at varying values of those crucial parameters, a diffusion coefficient $D$ may be extracted. In this research when diffusion techniques are used we vary only the gradient strength $g$ at fixed $\delta$ and $\Delta$.

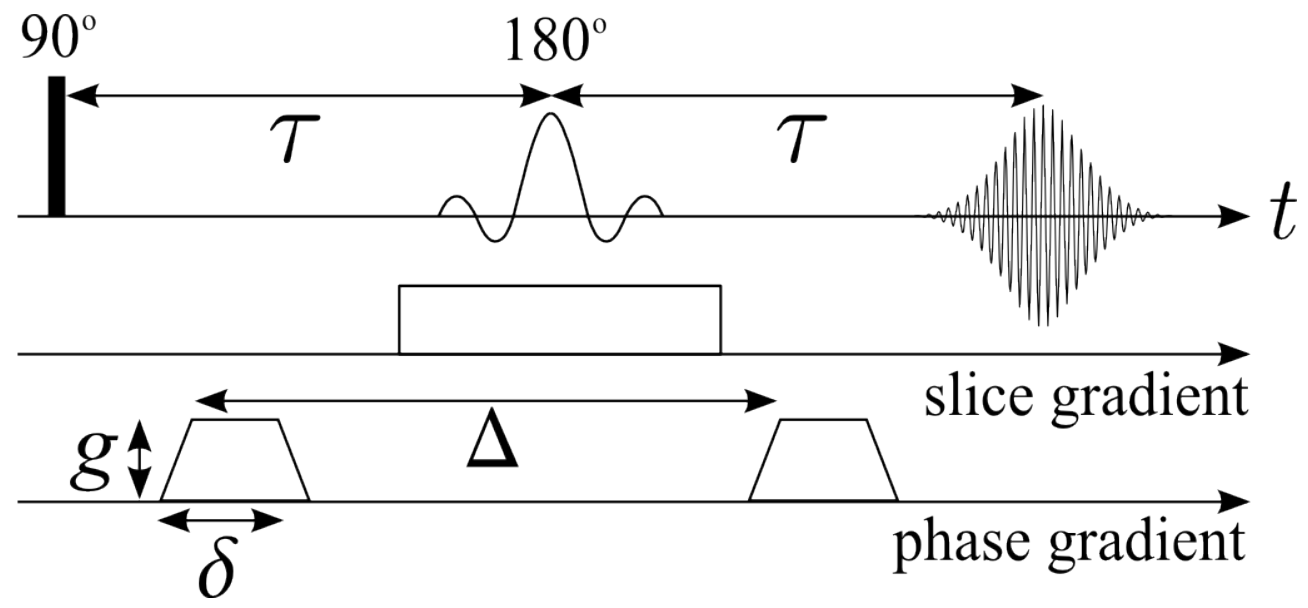

Figure 3.7: The PGSE experiment pulse program, using a soft refocussing pulse for spatial selectivity.

Attenuation data can be recorded for each spectral component of an NMR spectrum, creating a two-dimensional dataset (a DOSY spectrum [16]) from which unique diffusion coefficients can be extracted for different molecular components of the sample. This can be of particular use to us in helping identify molecular species within a standard 1D NMR spectrum, as the Stokes-Einstein relation of kinetic theory relates diffusion coefficient and molecular size (or subdomain size, e.g. in an emulsion).

\subsubsection{Magnetic Resonance Velocimetry}

In a very similar way to the discovery that the "real" space covered by position vectors $\mathbf{r}$ and "reciprocal" space covered by wave vectors $\mathbf{k}$ are Fourier conjugates, we can show that there is a space Fourier conjugate to displacements in spins: it is termed $q$-space. This is unsurprising, as a displacement vector is just another "real"-space vector $\mathbf{R}=\mathbf{r}^{\prime}-\mathbf{r}$, given a starting and ending position, 
and experimentally controlled time-of-flight. Implemented in the same spirit as the covering of $k$-space to obtain spatial spin density resolution, to perform basic velocimetry we need to cover $q$-space by using two field gradient pulses, the second is made to have the opposite sense of the first, which has the effect of "somewhat undoing" the effect of the first. In the instance where the effect is not completely negated, the spins have undergone such flow as to be located in a region of differing precessional frequency than in its original location. The spins have moved.

One respect which makes velocimetry different from imaging is the lack of uniqueness amongst spins as to the zero-point of this displacement-space. Spins at different locations can undergo the same displacement within the observation time and contribute the same effect to the signal. Because of this we need to introduce an average propagator, $P_{s}(\mathbf{R}, t)$, a device specifying the proportion of spins that underwent a certain diplacement, expressed as a normalised probability distribution function,

$$
P_{s}(\mathbf{R}, t)=\int P_{s}(\mathbf{r} \mid \mathbf{r}+\mathbf{R}, t) \rho(\mathbf{r}) d \mathbf{r}
$$

The average propagator describes the probability that any molecule will have a displacement $\mathbf{R}$ at time $t$. It is a probability function common to all spins within the active volume, and through the integral shown, calculated through the propagator of each individual spin $P_{s}(\mathbf{r} \mid \mathbf{r}+\mathbf{R}, t)$ and the spin density $\rho(\mathbf{r})$ at the reference time $t=0$.

\subsubsection{The standard pulsed-gradient spin-echo velocime- try experiment}

The standard method of obtaining a two-dimensional velocity map is to utilise a pulse sequence incorporating a pulsed-gradient spin-echo technique combined with a two-dimensional imaging technique. As we have seen in the previous section, the application of two magnetic field gradient pulses in opposite senses based around an echo-forming pulse (either "hard" or "soft") results in a average propagator being generated. This propagator is in fact a sum of all contributions from spins undergoing motion within a certain volume, and in the case 
of velocimetry imaging, only from within a certain voxel. As such the average propagator has characteristics relating to the pure, homogeneous flow of the spins in the fluid, as well as the random Brownian motions arising from diffusion. With such a three-dimensional configuration (two spatial dimensions conjugate to $k$-space, one velocity dimension conjugate to $q$-space) it is rare to inspect the signal at a great number of $q$-gradient values. The simplest reason for this is that in the convolution of the propagators from flow and diffusive components, the maximal value of the average propagator should occur at the very same position in the velocity spectrum as for flow, owing to the symmetric nature of diffusive mechanisms. It is often of more interest (particularly in complex fluids research) to produce the most spatially resolved image displaying velocity information, than to compromise in this respect. In the analysis of the $q$-space data, zero-filling data points located past the limited number of $q$-steps is done for resolution reasons.

A simple pulse program is shown in fig. 3.8, demonstrating the PGSE and imaging gradient components commonly used. It is important to note than any two modular components of pulse programs which yield a propagator and an imaging sequence can be used, as we shall see in section 3.8.8.

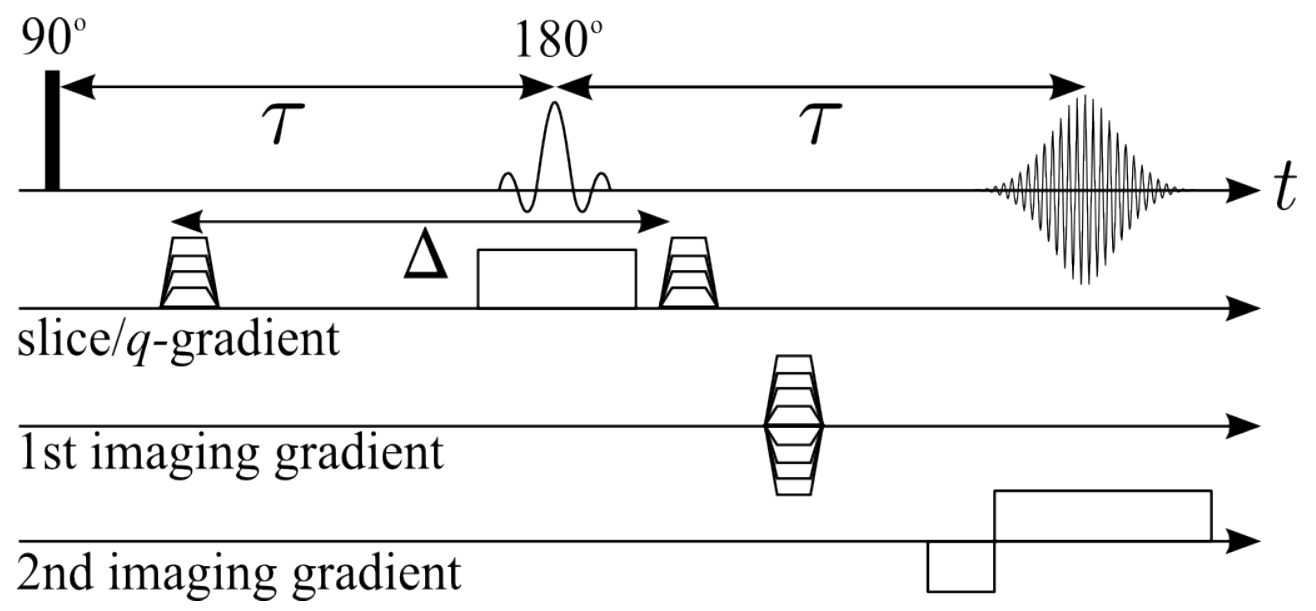

Figure 3.8: A simple velocity mapping pulse program, incorporating a $q$ encoding gradient in a single direction, and two $k$-encoding gradients in two different spatial directions.

Automation of the analysis process following this experiment is a procedure which takes the average propagator for each voxel, and extracts the modal ve- 
locity value in each, for display as a colour map. As a final note, it should be remembered that the direction in which the magnitude of the velocity is measured in this experiment may be selected independently of the orientation of the imaging plane. It is therefore possible in principle to map a three-dimensional velocity vector field over the volume of interest, by using three collaborative experiments.

\subsubsection{Fast imaging of velocimetry with RARE}

By incorporating a string of rf echo pulses into a pulse sequence, known as the CPMG (Carr-Purcell, Meiboom-Gill) train [17-19], signal that would normally decay following the acquisition period and be lost, can be refocussed, albeit modified through further normal relaxation processes. In using such a technique, rather than traversing through $k$-space one line per excitation, the whole plane of $k$-space may be covered in a single shot through intelligent placement of incremental phase gradients in the time between echoes. This technique is known as RARE imaging (Rapid Acquisition with Relaxation Enhancement). Combining velocimetry techniques with the RARE sequence, the flexibility and the potential of combining magnetic resonance techniques becomes apparent. In fig.3.9, a CPMG train being used as the basis of RARE imaging is set in place following a velocity encoding pair of gradient pulses sandwiching another echo pulse.

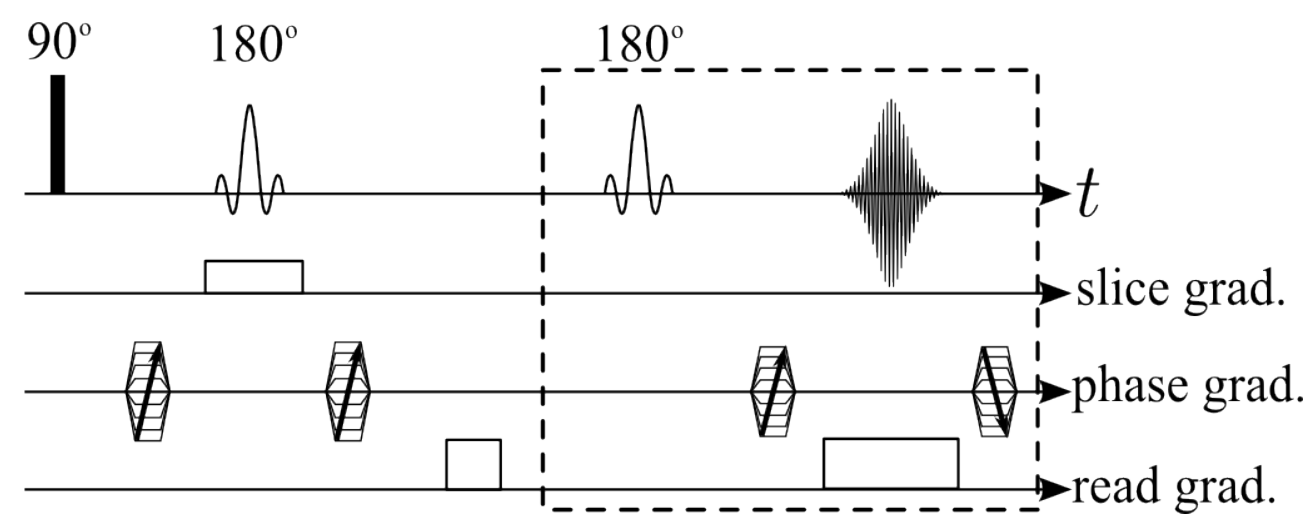

Figure 3.9: A PGSE-type velocity encoding step follows the initial $90^{\circ}$ r.f.pulse, where the gradient pulses may actually be placed on any of the three gradient directions to encode as desired. The dotted line encloses the RARE imaging sequence, which is looped over to traverse $k$-space. 
The major benefits of this pulse sequence for this research, besides saving experimental time, is the potential to begin to achieve realistic temporal resolution in observing velocity fluctuations in materials under flow conditions. To achieve this, however, a given sample must have magnetic resonance $T_{2}$ relaxation times such that the signal lasts adequately far through the CPMG train. Imaging artefacts such as ghosting are also more readily apparent in such experiments, although the use of only every second echo is certainly some help in this regard, negating the so-called 'even-odd echo effect'.

It should be noted that while the imaging sequence is much reduced in duration thanks to the echo train, the $q$-space encoding performed by the PGSE component of the experiment is still carried out in the normal way, i.e. $q$-space is still traversed through successive scans incrementing the integral of the gradient strength during the pulse through the variation of $g, \delta$ and $\Delta$.

\subsection{The Relevance of Magnetic Resonance Tech- niques in Rheology}

Most importantly, we have seen that magnetic resonance is a rich field providing flexible techniques to the experimenter. By applying radio-frequency oscillating magnetic fields to a sample bathed in a polarising magnetic field, we can perform countless experiments. These vary from the simple pulse-acquire sequence required to obtain spectral properties determined by the nuclear spin Hamiltonian in place, through to imaging experiments designed to highlight how fast a material is moving within a certain region, and beyond.

From the perspective of conjoining magnetic resonance and rheology - the field known as Rheo-NMR - it is the superposition of many techniques within a single experiment that yields the most valuable information. The non-invasive nature of NMR facilitates progress towards understanding the mechanical properties of materials by correlating the controlled deformation of the sample with experimentally observed local strain rate (velocimetry) and molecular alignment (spectroscopy) over the entire volume (imaging). This understanding is exciting whether the material under investigation be homogeneous and Newtonian, or be a complex fluid that at first glance seems pathological. 


\section{Bibliography}

[1] F Bloch, WW Hansen, and M Packard. The nuclear induction experiment. Physical Review, 70(7-8):474-485, 1946.

[2] EM Purcell, HC Torrey, and RV Pound. Resonance absorption by nuclear magnetic moments in a solid. Physical Review, 69(1-2):37-38, 1946.

[3] A Abragam. The Principles of Nuclear Magnetism. Clarendon Press, Oxford, 1961.

[4] PT Callaghan. Principles of Nuclear Magnetic Resonance Microscopy. Oxford University Press, Oxford, 1991.

[5] R Kimmich. NMR Tomography Diffusometry Relaxometry. Springer, Heidelberg, 1997.

[6] B Blumich. NMR Imaging of Materials. Oxford University Press, New York, 2000.

[7] CP Slichter. Principles of Magnetic Resonance. Springer-Verlag, Heidelberg, 1990.

[8] G.B. Arfken \& H.J. Weber. Mathematical Methods for Physicists, 5ed. Harcourt Academic Press, 2001.

[9] DI Hoult and RE Richards. Signal-to-noise ratio of nuclear magneticresonance experiment. J. Magn. Reson., 24(1):71-85, 1976.

[10] Y Zur and S Stokar. A phase-cycling technique for canceling spurious echoes in NMR imaging. J. Magn. Reson., 71(2):212-228, FEB 11987. 
[11] PC Lauterbur. Image formation by induced local interactions - examples employing nuclear magnetic resonance. Nature, 242(5394):190-191, 1973.

[12] P Mansfield and PK Grannell. NMR diffraction in solids. Journal of Physics C-Solid State Physics, 6(22):L422-L426, 1973.

[13] EL Hahn. Spin echoes. Physical Review, 80(4):580-594, 1950.

[14] PD Majors and A Caprihan. Fast radial imaging of circular and spherical objects by NMR. J. Magn. Reson., 94(2):225-233, SEP 1991.

[15] EO Stejskal and JE Tanner. Spin diffusion measurements - spin echoes in presence of a time-dependent field gradient. J. Chem. Phys., 42(1):288, 1965.

[16] KF Morris and CS Johnson. Resolution of discrete and continuous molecular-size distributions by means of diffusion-ordered $2 \mathrm{~d}$ NMRspectroscopy. J. Am. Chem. Soc., 115(10):4291-4299, MAY 191993.

[17] HY Carr and EM Purcell. Effects of diffusion on free precession in nuclear magnetic resonance experiments. Physical Review, 94(3):630-638, 1954.

[18] S Meiboom and D Gill. Modified spin-echo method for measuring nuclear relaxation times. Rev. Sci. Instrum., 29(8):688-691, 1958.

[19] S Meiboom, Z Luz, and D Gill. Proton relaxation in water. J. Chem. Phys., 27(6):1411-1412, 1957. 


\section{Chapter 4}

\section{Transverse Relaxation in Sheared Polymer Melts}

\subsection{Introduction}

As we have seen in Chapter 2, polymers are macromolecules which are the connected sum of many small, simple chemical components. While not always the case, they very often have the topology of a flexible one-dimensional object, i.e., by moving from one end of a polymer to another through chemical bonds (usually carbon-to-carbon bonds), one does not come across loops or major branches. To a great degree the one-dimensional nature of these linear polymers outweighs the chemical functionality of sub-units along the length of the polymer, and as such the dynamics of polymers in general can be modelled in much simpler and generic ways. Despite the omission of chemical properties from many physical models of polymers, the breadth of parameters which affect polymers behaviours is large: concentration, temperature, solvent effects and chain flexibility are important variables in their description.

In this chapter the timescales over which dynamics occur within linear polymers in the so-called melt phase (concentrated polymers which, while being highly viscous, are fluid without the presence of a solvent) are explored. It will be seen that their effect on the transverse relaxation of spin coherence is sufficiently large that very simple proton NMR experiments may be used to extract orientational information in sheared polymer melts. 


\subsubsection{Motivation}

In the first instance, deuteron NMR is the gold standard in studying orientational and ordering phenomena, as we shall see in all aspects of ordering in the remainder of this thesis. However, proton magnetic resonance is not totally a lost cause in this regard. Nakatani et al. [1] set out to observe changes in the proton NMR line shape for a polysiloxane melt undergoing steady shear flow, explaining that this effect may arise due to incomplete directional averaging in segmental reorientation, leading to a residual dipolar proton-proton interaction for protons in the mean-field of the polymer tube. While this experiment failed for various reasons, the field of Rheo-NMR came into being, in a spectroscopic sense. In the interim, deuteron NMR has held sway in spectroscopic investigations of alignment in materials, through the use of the direction-dependent electric quadrupole interaction, which in experiment may be experienced by deuterons bound and native to the aligned phase, or through the presence of a (potentially plasticising) deuterated probe molecule. The first scenario is in general expensive to arrange; the second may be detrimental to the rheology for both chemical and physical reasons. So as to show that Rheo-NMR can be used successfully without deuterons, we numerically validate an approach to the analysis of proton-proton spin correlations in entangled polymer fluids [2], by using previously gained experimental relaxation data from Dr. Ryan Cormier [3], and by tuning the parameters appropriate to the sample, which is being sheared continuously in a cylindrical Couette flow.

By calculating the expected proton-proton dipolar interactions generated through such residual anisotropy, in conjunction with polymer dynamics as described by the Doi-Edwards [4] approach to polymers based on the tube model and reptation, the model predicts transverse relaxation which can be matched with the experimental results of a simple spin-echo NMR pulse sequence.

\subsubsection{Approach and Method}

By first assuming an average dipolar interaction strength common to all protons undergoing equilibrium dynamics, given by the second moment, $\omega_{d}^{2}$, of the dipolar frequency distribution experienced by these protons, we arrive at the first of the three main tunable parameters we shall see are so important to the 
numerical fitting of model to data. However, with the expectation that local fluctuations in stretching and bunching of the polymer in accordance with the standard Gaussian distribution of end-to-end distances, we shall allow a distribution of $\omega_{d}^{2}$, appropriately summing contributions.

Secondly, we use the Doi-Edwards properties of reptating polymers existing within a network of topological constraints to determine the probabilities of a certain proton bound to the polymer returning to the same segment of tube at time, $t$. This is the only section of the polymer tube we know to be correlated with the original host segment in direction at such a time. This return-toorigin (RTO) probability is vital in the calculation of the correlation function for residual dipole interactions, and depends on the tube disengagement time, $\tau_{d}$, and the number of tube steps, $Z$, typically encountered along the contour length of the polymer (being the molecular mass of the polymer divided by the mass of polymer located between two entanglements). The Doi-Edwards model is then also used to determine the angular distribution of tube-step orientations for a polymer undergoing shear, in order to project each tube-step contribution into the laboratory Zeeman frame, this distribution is obtained following the assumption of the zero-shear standard isotropic distribution governed by the second rank Legendre polynomial $\frac{1}{2}\left(3 \cos ^{2} \theta-1\right)$, with $\theta$ being the polar angle between tube segment and magnetic field.

Finally, we sum all contributions to the NMR signal coming from protons residing in individual steps, a fundamental point of difference to deuterated probe molecule experiments, in which the probe molecule experiences a timeaveraged contribution to its fleeting interactions with the whole sample nearly ergodically.

\subsection{Theory}

Nuclear spins attached to a polymer undergo rapid reorientational motions in accordance with the Rouse dynamics seen previously, and as such it is our job first to describe the nuclear interactions that such a spin species is subject to. The Rouse dynamics involved here are constrained by the tube, a mean-field generated by the envelope of the encapsulated step-like space found by weaving a path through a network of (for now, at least) fixed obstructions through which 
the polymer may not pass. As the polymer reptates back and forth in a onedimensional, curvilinear mode of diffusion through this envelope, orientational coherence is lost for a particular spin, in all cases except for the one in which it returns to the identical segment in which it was located when observation began. The characteristic length, $a$, of a tube step before encountering an entanglement is our basic unit of measurement.

As such, there are three clearly delineated characteristic times which will be important in applying a model of dipolar correlation to polymers: (a) the time for a proton to diffuse somewhat freely before encountering the tube wall, at which point its diffusion becomes restricted. This is known as the equilibration time, $\tau_{e}$, (b) the time associated with a balancing of the equilibration modes of motion and the curvilinear motion of the polymer as a whole is called the Rouse time, $\tau_{R}$, and (c) the time taken for a polymer to diffuse (on average, with curvilinear diffusion coefficient $D_{1}=k_{B} T / N \zeta$ ) a sufficient distance such that the original tube is largely lost, the tube disengagement time, $\tau_{d}$. It is this final relaxation time associated with tube dissipation which is truly detrimental to the return-to-origin probability of a proton.

Strictly following the Doi and Edwards theory, these three times are given by

$$
\begin{gathered}
\tau_{e}=\tau_{R} / Z^{2} \\
\tau_{R}=R^{2} / 3 \pi^{2} D_{1} \\
\tau_{d}=3 Z \tau_{R}
\end{gathered}
$$

\subsubsection{Dipolar relaxation function for reptating polymer segments in zero shear}

As we have seen, the dipolar contribution to the nuclear Hamiltonian by the interaction between one proton and another is vastly outweighed by the Zeeman term arising from the external magnetic field. As such the off-diagonal components of the internuclear, homonuclear dipolar interaction operator are 
completely ignored in this model, themselves only contributing to longitudinal spin-lattice relaxation. It is the diagonal terms in the sum of all the throughspace interactions which will determine the precessional phase distribution of the nuclear spins and hence will play a dominant role in determining the spinspin relaxation in the polymer melt.

Formed by the inner product of rank-2 spatial and spin operators, the Hamiltonian contribution of the interaction between two spins labelled 1 and 2, separated by an internuclear distance $r_{12}$ can be written as

$$
H_{D}(t)=\frac{-\mu_{0} \gamma^{2} \hbar}{4 \pi r_{12}^{3}} \sum_{m}(-1)^{m}\left(\frac{24 \pi}{5}\right)^{1 / 2} Y_{2}^{m}(\Theta(t), \Phi(t)) T_{2}^{m}
$$

where $Y_{2}^{m}$ are the spherical harmonics of order 2 and component $m$, and the $T_{2}^{m}$ are bilinear products of spin operators [5] which transform as spherical tensors. The angles $\Theta$ and $\Phi$ refer to the orientation of the internuclear vector with respect to the referential main polarising field, and as such will fluctuate as the spins diffuse about throughout all the characteristic timescales.

The secular components of this dipolar perturbation to the Hamiltonian influence the precessional frequencies of spin coherences, contributing to the transverse relaxation associated with dephasing. This secular part of the interaction can be written as

$$
H_{D 0}(t)=\frac{-\mu_{0} \gamma^{2} \hbar}{4 \pi r_{12}^{3}} P_{2}[\cos \Theta(t)]\left[3 I_{1 z} I_{2 z}-\mathbf{I}_{1} \cdot \mathbf{I}_{2}\right]
$$

where $P_{2}(\cos \Theta)$ is the second order Legendre polynomial $\frac{3}{2}\left(\cos ^{2} \Theta-\frac{1}{3}\right)$. Dipolar perturbations such as these induce additional precession, $\omega(t)$, with respect to the Larmor frequency, $\omega_{0}$. It is only this additional relative precession that will be considered in the following work.

Through equilibration motion, the conformation of a polymer within a tube lying naturally between entanglements may be freely chosen. In addition, the contiguous tube steps of length, $a$, of which the tube is comprised, are randomly oriented in thermal equilibrium. Each of these steps may be labelled with a normalised vector indicating its direction (see fig. (4.1)) 


$$
\hat{\mathbf{u}}=\left(\begin{array}{c}
\sin \theta \cos \phi \\
\sin \theta \sin \phi \\
\cos \theta
\end{array}\right)
$$

where the spherical polar angles are measured with reference to the magnetic field frame as the $z$-axis, and some arbitrary perpendicular direction as the $x$-axis, a reference frame we shall later refer to as the $\{x, y, z\}$ or simply the laboratory frame. Ultimately it is against this frame that any projections will be made in our simulations and experiments.

Equilibration modes allow monomers to tumble at much faster rates than the dipole interaction strength, and as such the dipolar interaction is "pre-averaged" within the local constraining tube step. It is these residual dipolar interactions that will allow investigation into slow polymer dynamics by NMR. Let the angles $(\theta, \phi)$ denote the orientation of the tube step vector, $\mathbf{u}$, with respect to the magnetic field and $\left(\Theta_{\mathbf{u}}, \Phi_{\mathbf{u}}\right)$ the orientation of the fluctuating internuclear vector with respect to $\mathbf{u}$. Then, under motional averaging conditions, and by the spherical harmonic addition theorem, it may be shown that

$$
P_{2}(\cos \Theta(t))=\left\langle P_{2}\left(\cos \Theta_{\mathbf{u}}(t)\right)\right\rangle P_{2}(\cos \theta(t)) .
$$

Pre-averaging results in $\left\langle P_{2}\left(\cos \Theta_{\mathbf{u}}(t)\right)\right\rangle$ being small but finite, leading to an effectively scaled dipolar interaction strength determined by the local value of $P_{2}(\cos \theta(t))$, where we note that $\cos \theta(t)=\hat{u}_{z}(t)$. Allowing for this scaling effect we may write

$$
\omega(t)=\omega_{d}\left(\hat{u}_{z}^{2}(t)-\frac{1}{3}\right)
$$

where $\omega_{d}$ represents the strength of the pre-averaged dipolar interaction.

As the polymer reptates within the tube, the segments experience stochastic fluctuations which arise from different projections, $\hat{u}_{z}(t)$ of the local tube directors. This leads us to describe $\omega(t)$ in terms of its two-point correlation function

$$
C(t)=\langle\omega(t) \omega(0)\rangle
$$


and its initial value, $\omega_{d}^{2}$.

Up to this point we have considered only a local spin pair experiencing dipolar interactions. Despite this, $\omega_{d}$ can encompass multiple dipolar interactions, whether intra- or inter-molecular, so that $\omega_{d}$ represents the r.m.s. intensity of the combined set of dipolar interactions. It seems reasonable to assume that reptation will cause similar fluctuations in both intra- and inter-molecular components. It is important to note that motional averaging, expressed through eqn.(4.7), causes the effective direction of the dipolar interaction to be successively projected. This means that all fluctuations faster than the strength of the dipolar interaction (i.e., all internal segmental motion of the polymer) cause the effective inter-nuclear axis to be the tube step direction (4.1)).

It is important to note that even allowing for multiple spin interactions, the scaled interaction strength $\omega_{d}$ will not be identical in each tube step because the distribution of lengths of sub-chains in each tube step will be Gaussian. It may be shown [6] that the dipolar interaction strength for a random coil sub-chain of $K \mathrm{Kuhn}$ segments of length, $b$, and end-to-end length, $r_{K}$, is given by

$$
\omega_{d}=\frac{r_{K}^{2}}{K b^{2}} \omega_{d 0}
$$

where $\omega_{d 0}$ is the root mean square $\bar{\omega}_{d}^{2}$ of the distribution of dipolar interaction strengths.

The probability distribution $P\left(r_{K}\right) \sim r_{K}^{2} \exp \left(-\frac{3 r_{K}^{2}}{2 K b^{2}}\right)$ fully determines the probability distribution $P\left(\omega_{d}\right)$ of dipolar interaction strengths, whether arising from intra- or inter-molecular interactions. This effect will need to be accounted for in our theory of dipolar interaction fluctuations.

\section{Dipolar correlation and transverse relaxation in the Doi-Edwards, De Gennes model}

The NMR transverse relaxation experiment to be described here was carried out using a spin echo sequence which has the effect of refocussing all unwanted magnetic field inhomogeneities whose Hamiltonian terms are all linear in the spin operators, while leaving unaffected the bilinear dipole-dipole interaction. What is measured therefore is 


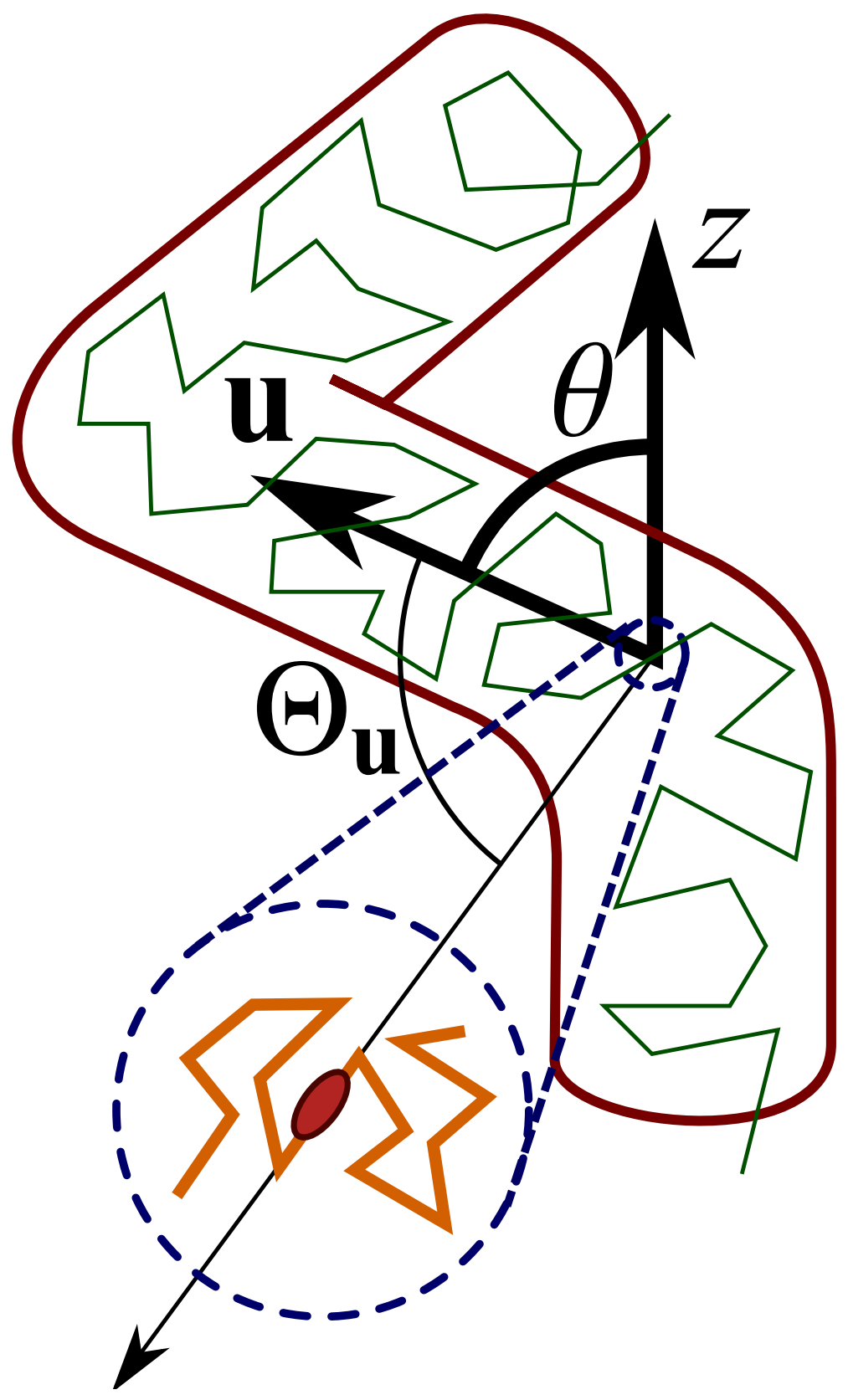

Figure 4.1: (a) Polymers are free to diffuse within a tube formed by the surrounding network. Characteristic steps pointing in the directions $\{\mathbf{u}\}$ are randomly oriented under zero shear, though when the material is deformed (eg. by a shear stress) the tubes are much more likely to have greater alignment with some preferred direction. 


$$
R(\tau)=\left\langle\exp \left\{i \int_{0}^{\tau} d t^{\prime} \omega\left(t^{\prime}\right)\right\}\right\rangle
$$

Following the work of Anderson and Weiss, in which it is assumed that the distribution of frequencies that have arisen due to dipolar fluctuations is Gaussian for certain classes of experiment the normalised transverse relaxation function for a single proton located in a tube segment which provides a certain remnant dipolar interaction strength may be written as [5]

$$
R\left(\tau ; \omega_{d}\right)=\exp \left(-\int_{0}^{\tau} d t^{\prime}\left(\tau-t^{\prime}\right) C\left(t^{\prime}\right)\right)
$$

Now the NMR signal enveloped by the total relaxation can be determined by summing the signals from all protons, which experience a range of dipolar interaction strengths

$$
S(\tau)=\int_{0}^{\infty} d \omega_{d} R\left(\tau ; \omega_{d}\right) P\left(\omega_{d}\right)
$$

Clearly evaluation of $C\left(t^{\prime}\right)$ is the crucial step for any model of the dynamics. In the present case we may combine eqns.(4.8) and (4.9) to write

$$
C\left(t^{\prime}\right)=\omega_{d}^{2}\left[\left\langle\hat{u}_{z}^{2}\left(t^{\prime}\right) \hat{u}_{z}^{2}(0)\right\rangle-\frac{2}{3}\left\langle\hat{u}_{z}^{2}(0)\right\rangle+\frac{1}{9}\right]
$$

where we have assumed stationarity, $\left\langle\hat{u}_{z}^{2}\left(t^{\prime}\right)\right\rangle=\left\langle\hat{u}_{z}^{2}(0)\right\rangle$, and also that the local interaction strength, $\omega_{d}$, is unchanged from time 0 to $t$. Strictly, $\omega_{d}$ will change over a Rouse time, as local polymer segments reptate, though through the following reasoning we found this effect to be of minor significance.

A distribution of monomers located in neighbouring polymer tube segments may lead to a different number of Kuhn segments $K$ and $K^{\prime}$ being resident in each, which will lead to a variable local flexibility of the polymer. The total contribution from each tube step must be found, and so each tube step's contribution would need to be weighted appropriately. Alternatively we could simply choose an equal number, $K$, of Kuhn segments to lie in each segment, and assume that the mean orientation of these Kuhn segments is along the tube step director.

The end-to-end length of the $K$ segments is given by the Gaussian function 
$\Phi\left(r_{K}\right) \sim r_{K}^{2} \exp \left(-3 r_{K}^{2} / 2 K b^{2}\right)$ and the mean dipolar interaction strength felt by each would be $\omega_{d}\left(r_{K}\right)=\frac{r_{K}^{2}}{K b^{2}} \omega_{d 0}$. Calculating the NMR signal arising from a given segment with dipolar interaction strength $\omega_{d}\left(r_{K}\right)$ involves a correlation function $\langle\omega(0) \omega(t)\rangle$, where following equation 4.8 , is $\omega(t)=\omega_{d}\left(r_{K}(t)\right)\left[u_{z}^{2}(t)-\right.$ $1 / 3$. In considering what to do about the time dependence of the dipolar interaction strength, we assume it is uncorrelated to $u_{z}(t)$. Also $\omega_{d}\left(r_{K}(0)\right)$ and $\omega_{d}\left(r_{K}(t)\right)$ are uncorrelated due to local fluctuations in $r_{K}(t)$ being of the Rouse timescale, which is longer than the segmental diffusion yet much longer than the NMR timescale. Despite this, each local tube segment dipolar interaction strength is well-defined before decorrelation due to diffusion. The discrepancy we therefore might face is the potential difference between the correlation functions

$$
\langle\omega(0) \omega(t)\rangle=\omega_{d}\left(r_{K}\right) \omega_{d}\left(r_{K}^{\prime}\right)\left\langle\left[u_{x}^{2}(0)-1 / 3\right]\left[u_{x}^{2}(t)-1 / 3\right]\right\rangle
$$

and

$$
\langle\omega(0) \omega(t)\rangle=\omega_{d}\left(r_{K}\right)^{2}\left\langle\left[u_{x}^{2}(0)-1 / 3\right]\left[u_{x}^{2}(t)-1 / 3\right]\right\rangle
$$

A numerical algorithm was implemented to discover the magnitude of this difference, and indeed the differences were extremely small, and as such, we make allowance for this factor by incorporating it into the overall distribution of $\omega_{d}$ values.

Ball, Callaghan and Samulski [2] adopted the Anderson-Weiss [7] approach to defining the relevant correlation function for the reptating polymer chains in terms of a Return-To-Origin probability. For the case where segments return to origin, $\left\langle\hat{u}_{z}^{2}\left(t^{\prime}\right) \hat{u}_{z}^{2}(0)\right\rangle=\left\langle\hat{u}_{z}^{4}(0)\right\rangle$ while otherwise $\left\langle\hat{u}_{z}^{2}\left(t^{\prime}\right) \hat{u}_{z}^{2}(0)\right\rangle=\left\langle\hat{u}_{z}^{2}\left(t^{\prime}\right)\right\rangle\left\langle\hat{u}_{z}^{2}(0)\right\rangle=$ $\left\langle\hat{u}_{z}^{2}(0)\right\rangle^{2}$. The Doi-Edwards model gives us the means by which the RTO probability may be estimated for entangled high molecular mass polymers. We may estimate the chance that by curvilinear diffusion a certain monomer will return after a time delay, $t$, to exactly the same tube step it originally occupied at time $t=0$, provided that tube step is not yet annihilated due to reptation. This probability, denoted $\Psi_{R T O}(t)$, can be approximated by the values [2] 


$$
\Psi_{R T O}(t)= \begin{cases}\left(t / \tau_{e}\right)^{-1 / 4} & \tau_{e}<t<\tau_{R} \\ \left(t / \tau_{e}\right)^{-1 / 4}\left(t / \tau_{R}\right)^{-1 / 4} & \tau_{R}<t<\tau_{d}\end{cases}
$$

These $t^{-\frac{1}{4}}$ and $t^{-\frac{1}{2}}$ scaling regimes are related to well-known power law signatures for reptation [8].

Clearly, any estimation of $C\left(t^{\prime}\right)$ concerns two probabilities, the RTO probability and the probability that a tube for a given polymer as seen from the reference state still exists. For a polymer segment to return to the initial tube step with the same director, the probability is $\psi_{\text {tube }} \Psi_{R T O}$ and $u_{z}^{2}(t)=u_{z}^{2}(0)$. Note the need for $\psi_{\text {tube }}$ in the product, the requirement that the tube survives. Conversely the probability that the monomer ends up in a different environment step is $1-\psi_{\text {tube }} \Psi_{R T O}$. This complementary probability allows for correlation loss due to either tube decay or to a failure for a monomer to return-to-origin, and should either of these two negative results occur, $\hat{u}_{z}^{2}(t)$ will be uncorrelated with $\hat{u}_{z}^{2}(0)$, causing $\left\langle\hat{u}_{z}^{2}(t)\right\rangle=\left\langle\hat{u}_{z}^{2}(0)\right\rangle$.

The correlation can hence be written as the weighted sum

$$
\begin{aligned}
C(t) & =\omega_{d}^{2} A \psi_{\text {tube }}(t) \Psi_{R T O}(t)+\omega_{d}^{2} B\left(1-\psi_{\text {tube }}(t) \Psi_{R T O}(t)\right) \\
& =\omega_{d}^{2}\left[(A-B) \psi_{\text {tube }}(t) \Psi_{R T O}(t)+B\right]
\end{aligned}
$$

where, following eqn.(4.14)

$$
\begin{aligned}
& A=\left\langle\hat{u}_{z}^{4}(0)\right\rangle-\frac{2}{3}\left\langle\hat{u}_{z}^{2}(0)\right\rangle+\frac{1}{9} \\
& B=\left\langle\hat{u}_{z}^{2}(0)\right\rangle^{2}-\frac{2}{3}\left\langle\hat{u}_{z}^{2}(0)\right\rangle+\frac{1}{9}
\end{aligned}
$$

In thermal equilibrium the distribution of $\mathbf{u}$ vectors is isotropic, $\left\langle\hat{u}_{z}^{2}(0)\right\rangle=\frac{1}{3}$ and $B=0$. Note the probability that the tube existing at time zero has survived until time $t$ is denoted $\psi_{\text {tube }}(t)$, which, as we saw in section 2.4.3, is a well-known, multi-exponential function of time [4]

$$
\psi_{\text {tube }}(t)=\sum_{p \text { odd }} \frac{8}{\pi^{2} p^{2}} \exp \left(\frac{-p^{2} t}{\tau_{d}}\right)
$$


where $\tau_{d}$ is the tube disengagement time.

\subsubsection{Dipolar relaxation function for reptating polymer segments in the presence of shear}

\section{Shear deformation tensor in the hydrodynamic frame}

To handle the case where the polymer melt is continuously deformed, we make the assumption that the tube segments in the original melt hold a one-to-one correspondence with the tube segments in the resultant state. Typically the directions of the vectors along the tube segments are modified, though we still require that the new directors $\left\{\hat{\mathbf{u}}^{\prime}\right\}$ be normalised. It is important to note that we assume that only the tube segments enveloping the actual polymer are deformed and biased in direction, and that no modification of local polymer dynamics occurs.

In a prototypical situation which can be related in the laboratory to a Couette shearing cell, for example, we can associate the $X$ - (or velocity) direction with the shear direction, where the $Y$ - and $Z$-directions are the velocity gradient and vorticity directions respectively. In this hydrodynamic frame $\{X, Y, Z\}$, arbitrary displacements and velocities are transformed from their static counterparts through the application of the deformation tensor (which, in steady-state, is no longer a function of the time of observation)

$$
\mathbf{E}(\gamma)=\left(\begin{array}{ccc}
1 & \gamma & 0 \\
0 & 1 & 0 \\
0 & 0 & 1
\end{array}\right)
$$

We shall be particularly interested in applying this tensor to the unit vectors representing tube segment directors. These directors, $\{\mathbf{u}\}$, must be expressed in the $\{X, Y, Z\}$ frame, which for the three cases in which one of the three hydrodynamic axes are aligned with the $z$-direction (the NMR $B_{0}$ field direction) can be obtained by a simple cyclical interchange of components.

In the deformed material, we need to express the degree to which the newly transformed tube steps project onto the magnetic field axis. This is done simply by applying the deformation tensor to the unit vectors representing the tube 
step directors, and renormalising the resultant vector:

$$
\hat{u}_{z}^{\prime 2}=\hat{u}_{\{X, Y, Z\}}^{\prime 2}=\frac{(\mathbf{E} \cdot \hat{\mathbf{u}})_{\{X, Y, Z\}}^{2}}{\|\mathbf{E} \cdot \hat{\mathbf{u}}\|^{2}} \equiv h_{\{X, Y, Z\}}(\gamma, \theta, \phi)
$$

Here we are defining three functions $h_{X}, h_{Y}$ and $h_{Z}$, the subscript of which refers to the specific hydrodynamic axis aligned with the laboratory magnetic field direction. These functions express the component along the magnetic field direction of the new, transformed tube step director. Section 4.2 .3 explicitly details their calculation and effect.

Following Doi and Edwards [4] we assign the effective strain, $\gamma$, to the Weissenberg number, $\dot{\gamma} \tau_{d}$, owing to the competition between the aligning influence of overall strain rate $\dot{\gamma}$, and relaxation of the polymer disengaging from its original tube over the timescale, $\tau_{d}$. We do not incorporate the effect of convected constraint release (CCR) [4], but its possible required inclusion in future work will be discussed as needed throughout this chapter. It is a modification to the basic Doi-Edwards theory of polymer relaxation, in which the rate of disappearance of the tube restrictions themselves is taken into account, as the polymers from which they are formed disengage themselves. The value of the modified (shortened) terminal relaxation time is given by

$$
\frac{1}{\tau_{\mathrm{CCR}}}=\frac{1}{\tau_{d}}+\dot{\gamma}\left\langle u_{X} u_{Y}\right\rangle
$$

\section{The correlation function under shear}

Should a shear field (or other deformational flow) be imposed upon the polymer, the transformed and renormalised directors are substituted with no further modification to this expression, due to the one-to-one correspondence between tube steps in the thermal equilibrium and flow deformed distributions. We may now use this expression for $h$ in the equations for $A$ and $B$

$$
\begin{aligned}
& A(\gamma)=\left\langle h^{2}(\gamma, \theta, \phi)\right\rangle-\frac{2}{3}\langle h(\gamma, \theta, \phi)\rangle+\frac{1}{9} \\
& B(\gamma)=\langle h(\gamma, \theta, \phi)\rangle^{2}-\frac{2}{3}\langle h(\gamma, \theta, \phi)\rangle+\frac{1}{9}
\end{aligned}
$$


though, unlike the zero shear case, $B \neq 0$. From eqn.(4.18),

$$
R\left(\tau ; \omega_{d}\right)=\exp \left\{-\omega_{d}^{2} \int_{0}^{\tau} d t(\tau-t)\left[(A-B) \psi_{\text {tube }}(t) \Psi_{R T O}(t)+B\right]\right\}
$$

This is an expression involving an integral which we expect to be simple to evaluate numerically in the case that we have calculated $A$ and $B$ for an experiment conducted at a given shear rate previously. The $\dot{\gamma} \tau_{d}$-dependence inherited from $A$ and $B$ is implicit in the calculation.

\subsubsection{Predictions for magnetic field along the velocity and velocity gradient directions}

In the NMR experiment, the $z$-axis is defined by the magnetic field direction. In Doi-Edwards theory the $\{X, Y, Z\}$ axes refer to the hydrodynamic frame. In practice we may select different axes in this frame via the magnetic field, by simply choosing our sample geometry appropriately. For example, we may choose $z \equiv Z$ by using a Couette cell geometry which is aligned with the NMR $B_{0}$ field (the 'vertical' Couette). In this case the relevant projection of $h$ is $h_{Z}$. Through the use of a "slice-selection" technique in which a signal may be acquired from a pre-determined segment of the sample, a horizontal Couette can be used as shown in fig.(4.2) so that the NMR $B_{0}$ field is projected along $X$ or $Y$ for the chosen segment. We now undertake to find expressions for $h_{X}$ and $h_{Y}$.

\section{Velocity direction $=B_{0}$ direction}

Focussing now on the case where the shear direction is coincident with the $B_{0}$ field direction, we should note that we will still use the same deformation tensor (i.e. the one that produces the transformation $X \rightarrow X+\gamma Y$ in the hydrodynamic frame), but the components of $\hat{\mathbf{u}}$ will be cyclicly interchanged in correspondence with the axes. In this geometry $(X \equiv z, Y \equiv x, Z \equiv y)$, then, $u_{z} \rightarrow u_{z}+\gamma u_{x}$, and the deformation tensor equation reads 

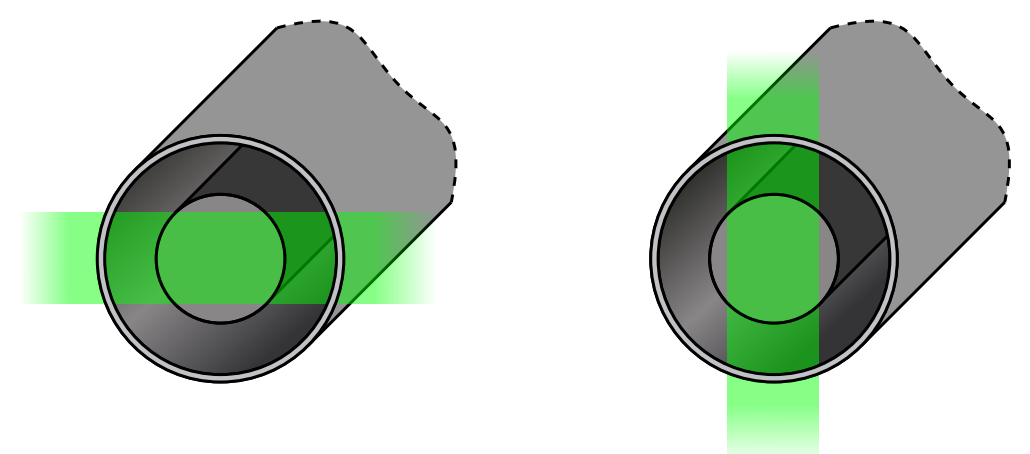

Figure 4.2: Simulating the NMR signal where the velocity direction $X$ is aligned with the magnetic field is equivalent to restricting the active volume a horizontal Couette cell to lie in the horizontal plane (left of figure), and (right of figure) vertical slice selection on a Couette cell is matched by the simulation $z \equiv Y$.

$$
\begin{aligned}
\mathbf{E} \cdot \hat{\mathbf{u}} & =\left(\begin{array}{lll}
1 & \gamma & 0 \\
0 & 1 & 0 \\
0 & 0 & 1
\end{array}\right)\left(\begin{array}{c}
\cos \theta \\
\sin \theta \cos \phi \\
\sin \theta \sin \phi
\end{array}\right) \\
(\mathbf{E} \cdot \hat{\mathbf{u}})_{z}^{2} & =(\mathbf{E} \cdot \hat{\mathbf{u}})_{X}^{2}=(\cos \theta+\gamma \sin \theta \cos \phi)^{2} \\
\|\mathbf{E} \cdot \hat{\mathbf{u}}\|^{2} & =1+\gamma \cos \phi \sin 2 \theta+\gamma^{2} \sin ^{2} \theta \cos ^{2} \phi
\end{aligned}
$$

and hence from eqn.(4.24)

$$
h_{X}(\gamma, \theta, \phi)=\frac{(\cos \theta+\gamma \sin \theta \cos \phi)^{2}}{1+\gamma \cos \phi \sin 2 \theta+\gamma^{2} \sin ^{2} \theta \cos ^{2} \phi}
$$

We are now free to use this expression in the spherical averages of eqns 4.26 and 4.27 to find $A(\gamma)$ and $B(\gamma)$, and hence continue to find $R(\tau)$, the modulation function of the NMR signal indicating transverse relaxation.

\section{Velocity gradient direction $=B_{0}$ direction}

We turn now to the final case which is orthogonal to the NMR frame, that in which the velocity gradient direction is coincident with the $B_{0}$ field. Although 
no numerically calculated relaxation data will be presented in this case, it is informative and interesting to investigate this geometry, in particular in the limiting cases of zero and infinite shear rates. Again we use the same deformation tensor ( $X \rightarrow X+\gamma Y$ in the hydrodynamic frame), and the components of $\hat{\mathbf{u}}$ will be cyclicly interchanged once more, such that $u_{y} \rightarrow u_{y}+\gamma u_{z}$, and the deformation tensor equation now reads

$$
\mathbf{E} \cdot \hat{\mathbf{u}}=\left(\begin{array}{lll}
1 & \gamma & 0 \\
0 & 1 & 0 \\
0 & 0 & 1
\end{array}\right)\left(\begin{array}{c}
\sin \theta \sin \phi \\
\cos \theta \\
\sin \theta \cos \phi
\end{array}\right)
$$

and similarly

$$
(\mathbf{E} \cdot \hat{\mathbf{u}})_{z}^{2}=(\mathbf{E} \cdot \hat{\mathbf{u}})_{Y}^{2}=\cos ^{2} \theta
$$

$$
\|\mathbf{E} \cdot \hat{\mathbf{u}}\|^{2}=1+\gamma \sin \phi \sin 2 \theta+\gamma^{2} \cos ^{2} \theta
$$

and hence from eqn.(4.24)

$$
h_{Y}(\gamma, \theta, \phi)=\frac{\cos ^{2} \theta}{1+\gamma \sin \phi \sin 2 \theta+\gamma^{2} \cos ^{2} \theta}
$$

\subsection{Experimental}

\subsubsection{Horizontal Couette Measurement of $T_{2}$ in Polymer Melts}

As explained in the introduction, the purpose of the present work is to use proton NMR transverse relaxation to measure segmental alignment under shear, thus circumventing the need for a deuterated probe molecule and the consequently lower signal-to-noise ratio. The through-space dipolar interactions between protons are analogous to the deuteron's electric quadrupolar interaction with the molecular bond electron cloud, so that the physics expressed by eqn.(4.5) apply in each case. The distribution of dipolar interaction strengths leads to a loss of phase coherence of the spin system and a damping of the signal, the so-called 
$T_{2}$ relaxation. To remove unwanted damping effects due to local field inhomogeneity, we utilise a spin echo to measure $T_{2}$. Under the spin echo, magnetic terms in the spin Hamiltonian are refocussed, while dipolar terms remain.

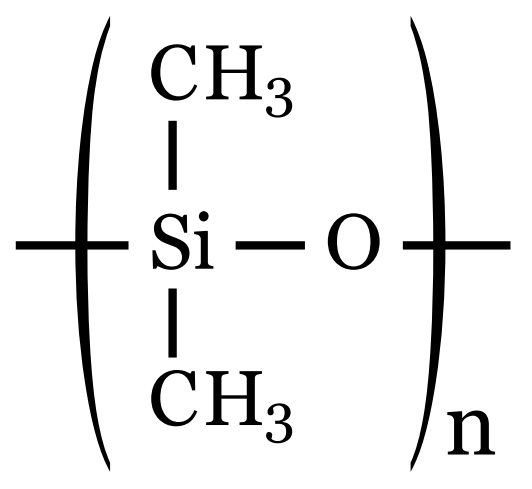

Figure 4.3: The chemical structure of poly(dimethylsiloxane).

NMR spectra and the extracted values for the characteristic transverse relaxation time $T_{2}$ were all obtained through the use of a horizontal Couette cell (see fig.(4.6)) located within the bore of a Bruker AMX $300 \mathrm{MHz}$ wide bore magnet. The sample used was a high molecular weight $\left(M_{p}=494 \mathrm{kDa}\right)$ poly(dimethylsiloxane) (or PDMS) (see fig.(4.3)) with a polydispersity index $M_{w} / M_{n}=1.84$, obtained from American Polymer Standards Corporation. PDMS has a glass transition temperature of approximately $-150^{\circ} \mathrm{C}$ [9], well below room temperature, and its structure (see figure 4.3) is such that both intra- and inter-molecular proton dipolar interactions play a role in the melt. The molecular weight located between entanglements $M_{e}$ in such a PDMS melt is $\sim 10 \mathrm{kDa}[9]$ and hence the number of entanglements $Z$ along the length of the polymer is simply $M / M_{e} \sim 49$. The flow curve of this polymer is shown in fig.(4.4) with both shear stress and normal stresses indicated.

The poly(dimethylsiloxane) is enclosed in a $0.5 \mathrm{~mm}$ gap between concentric MACOR machinable glass tubes. By using slice-selective gradients, only specific volumes within the sample that highlight the regions of interest (e.g., velocity direction aligned with the NMR $B_{0}$ field) are active in the experiment. This was achieved through the use of a sequence $\left(90^{\circ}{ }_{x}-\tau-180^{\circ}{ }_{y}-\tau-\right.$ acquire $)$, shown schematically in fig.(4.5). The selective excitation pulse sequence used was specially devised to minimise exposure of selected nuclear spins to any relaxation, so that high quality NMR spectroscopy can be performed in the 


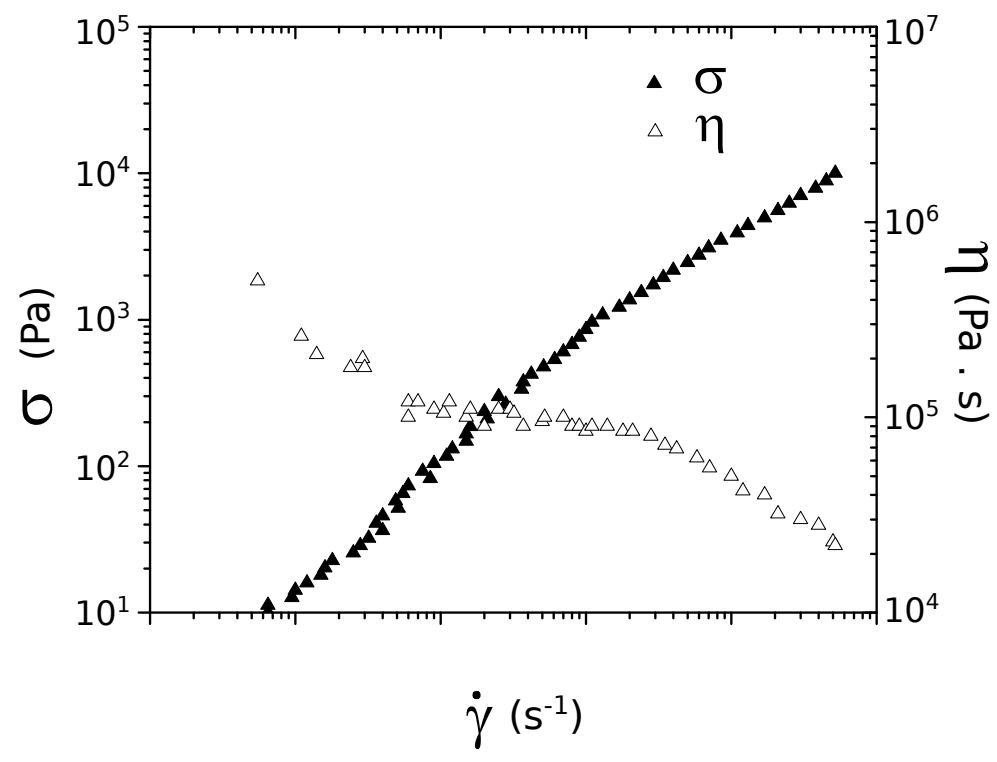

Figure 4.4: Flow curve for the PDMS sample used in all experiments.

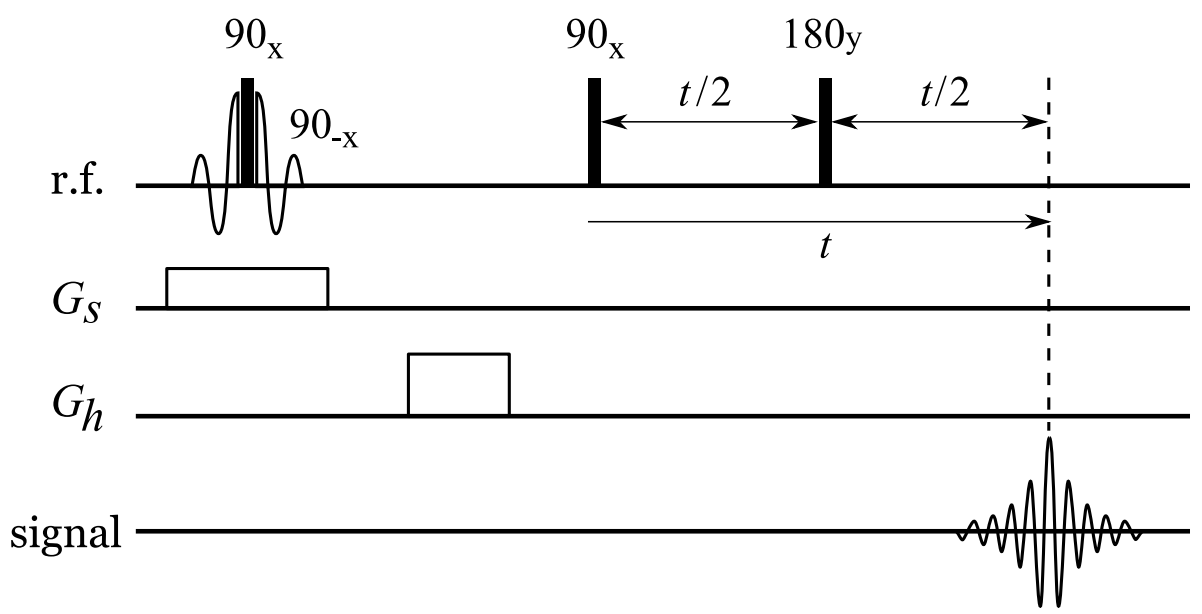

Figure 4.5: The Hahn echo pulse program, which consists of a preliminary pulse sandwich which leaves only longitudinal signal from a desired slice (achieved by applying a slice gradient $G_{s}$, followed by a transverse magnetisation-destroying homospoil gradient $G_{h}$ ), followed by a standard echo sequence of varying echo time. The signal strength as a function of echo time yields $T_{2}$. 

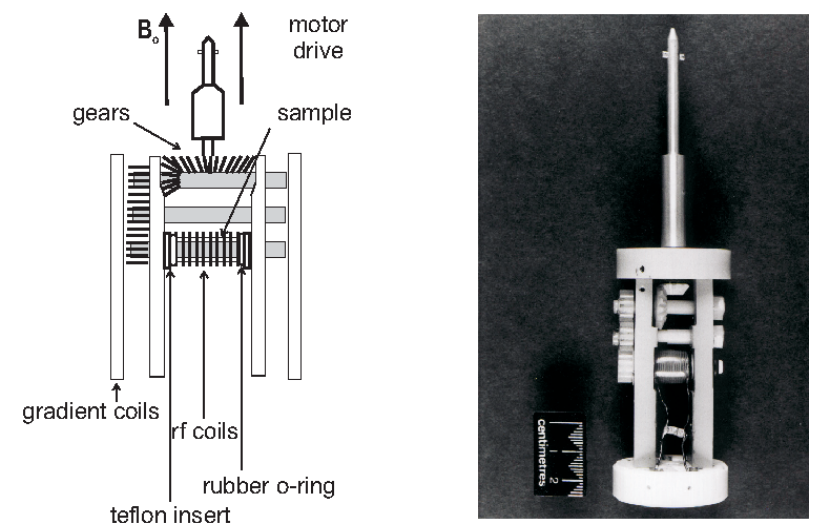

Figure 4.6: (From the thesis of R.J. Cormier) The horizontal Couette cell
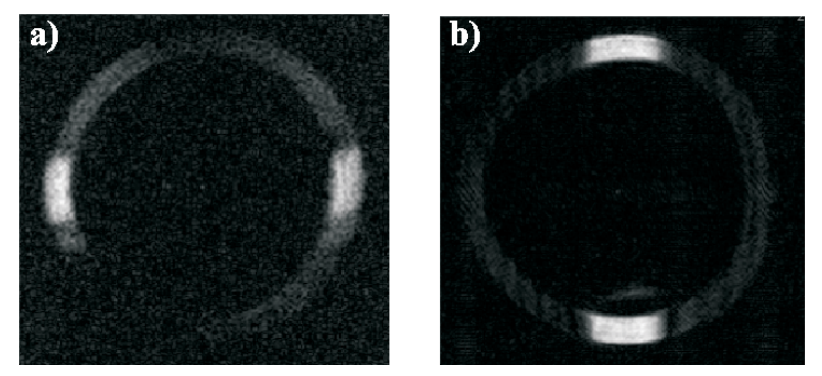

Figure 4.7: (From the thesis of R.J. Cormier) (a) Slice of active volume produced in a horizontal Couette cell (viewed along the cell vorticity axis) by applying a $z$-gradient in the pulse program, and (b) an alternate active volume produced by applying a gradient perpendicular to the $z$-direction and the vorticity axis of the cell 
desired region. This method was described by Kilfoil [10], however the essential details are shown in fig.(4.5). The technique employs a selective precursor pulse sandwich which destroys magnetisation outside the desired region but which stores along the $z$-axis for later recall, the magnetisation from the region of interest. Using a variant of the pulse sequence of fig.(4.5) this magnetisation can be used to obtain a confirmatory image (see fig.(4.7)). Experiments were carried out with an acquisition bandwidth of $50 \mathrm{kHz}$ and 102 different delay times $\tau$ were used between $0.1 \mathrm{~ms}$ and $300 \mathrm{~ms}$. The duration of the slice selective pulse is of the order of $1 \mathrm{~ms}$ so that insignificant slice distortion occurs due to shear. Furthermore, motion during the spin-echo does not significantly perturb the shear-dependent dipolar interactions due to the small proportion of fluid experiencing high velocity near the inner wall [11].

\subsubsection{Numerical Computation of $T_{2}$ in Polymer Melts}

Our numerical calculations based on the theory described previously were all performed by software hand-coded in $\mathrm{C}++$. The horizontal Couette with vertical slice selection (the $X \equiv z$ case) configuration is assumed as the basis of calculations. The 'experimenter' is required to be able to specify the shear rate applied, and the software created allows the choice of the two tunable parameters particularly relevant to the sample being investigated, namely the tube

disengagement time, $\tau_{d}$, the average dipole interaction strength, $\omega_{d 0}$, and $Z$, the number of tube segments the polymer is divided into by entanglements.

Given the orientation and shear rate, it is a simple matter to evaluate the relevant $h_{\{X, Y, Z\}}$ function, and hence find $A$ and $B$. The modification of eqn.(4.12) is appropriate at this stage so as to accommodate our expectation that the distribution of dipolar interaction strengths is not unimodal, but is spread broadly due to the end-to-end vector of local sub-chains in each tube being Gaussian. The double integral over $\omega_{d}$ and $t$ can now be evaluated to determine the normalised transverse relaxation function desired. 


\subsection{Results and Discussion}

\subsubsection{Experimental measurement of $T_{2}$ in polymer melts, and fitting model parameters}

Using the Hahn echo pulse sequence previously described, the echo signal acquired is modulated by the transverse relaxation envelope. Plotted on a logarithmic scale, all echo signal intensities acquired at shear rates ranging from 0 to $100 \mathrm{~s}^{-1}$ are closer to Lorentzian $(S(t) \sim \exp (-k t))$ than Gaussian $((S(t) \sim$ $\left.\exp \left(-k t^{2}\right)\right)$, as seen in fig.(4.8). By making a Taylor expansion of the returnto-origin probabilities in eqn.(4.12), it can be seen that if the problem were treated with a unique value of the pre-averaged dipolar interaction strength, a Gaussian lineshape might be expected. However, by performing the integral over the correct weighting of $\omega_{d}$ values, a lineshape closer to Lorentzian results. Note however that for very short times, the theory (see fig.(4.9)) does predict an opposite curvature to that seen in the measurements. One possible explanation for the initial rapid decay of the measured signal is the settling of the spin echo to steady state under the action of a less-than-perfect $180^{\circ}$ refocussing radio-frequency pulse.

Fig.(4.10) shows the extraction of $1 / e$ points from these acquired curves as a function of shear rate. From this data, $T_{2}$ appears to be heavily shear-ratedependent at low $(\dot{\gamma}<20)$ shear rates before finding an ultimate $T_{2}$ at higher shear rates, where little or no further decrease is seen.

In fitting the three tunable parameters: $Z, \omega_{d 0}$ and $\tau_{d}$; three characteristic reference conditions were used, namely the transverse relaxation times at $\dot{\gamma}=$ $0 \mathrm{~s}^{-1}, \dot{\gamma}=10 \mathrm{~s}^{-1}$ and $\dot{\gamma}=100 \mathrm{~s}^{-1}$. With the preliminary values of $Z=50$, $\tau_{d}=210 \mathrm{~ms}$, chosen from earlier work [12], the ratio of the transverse relaxation time at low and high shear rates was found to be disproportionately large and hence these values were adjusted to give a best fit between experimental values of $T_{2}$ and numerical simulation. We obtain 

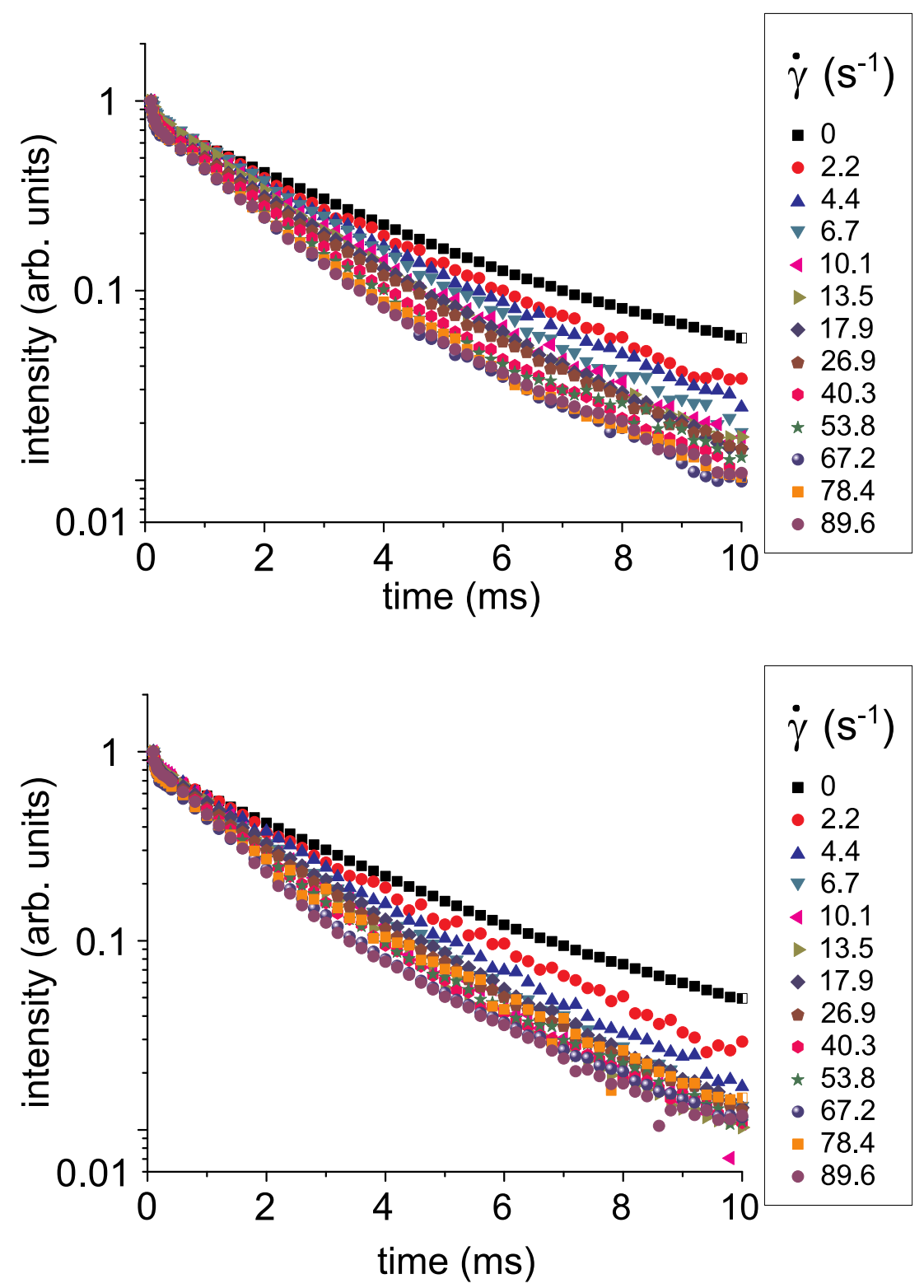

Figure 4.8: (a) Experimentally measured echo signals, having obtained a horizontal slice within the horizontal Couette geometry $(z \equiv X)$ for different shear values, and (b) those signals obtained through the use of a vertical slice 


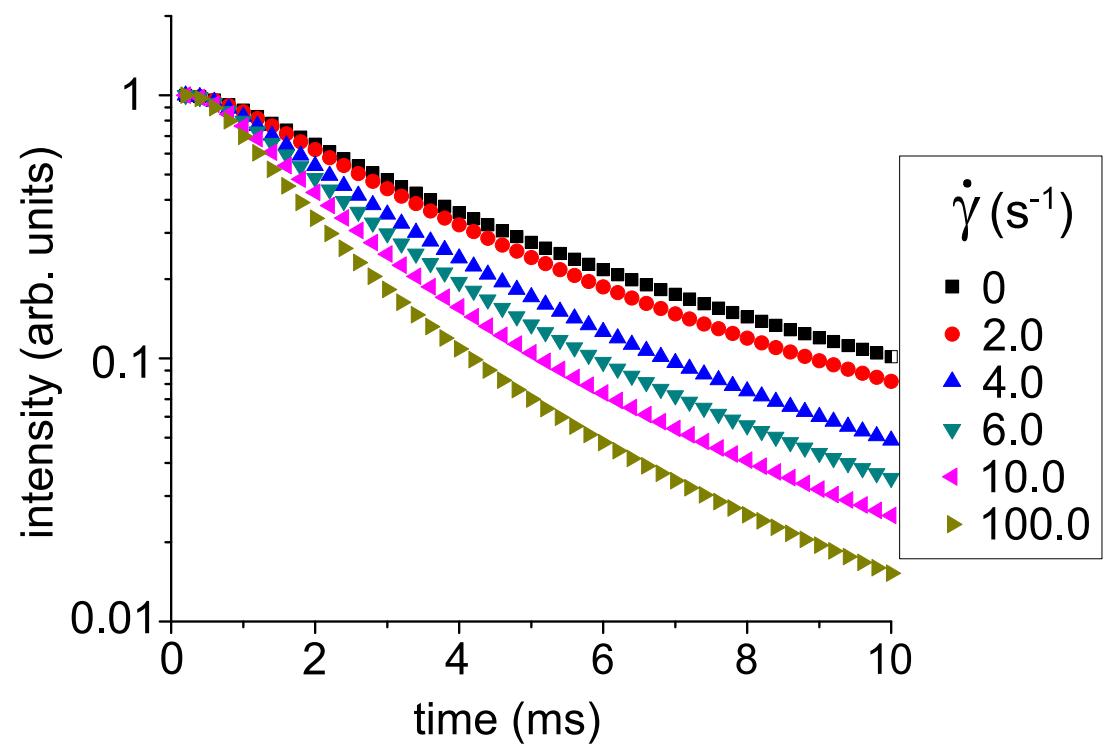

Figure 4.9: Simulated echo signals for the horizontal Couette geometry ( $z \equiv$ $X)$ for different shear values

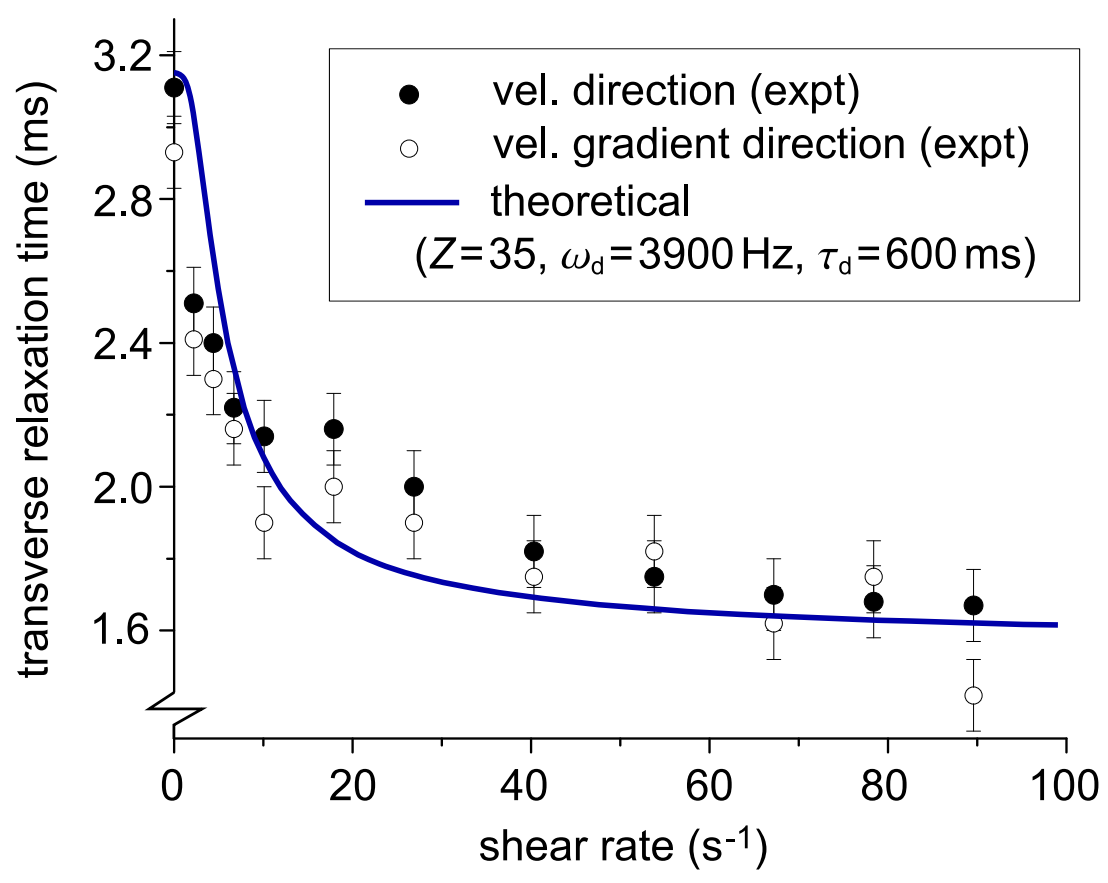

Figure 4.10: Simulated transverse relaxation time as a function of shear rate, in the case where the hydrodynamic $X$-axis is aligned with the magnetic field $B_{0}$. The data points correspond to $1 / e$ times obtained for the echo attenuation at different shear rates. 


$$
\begin{aligned}
Z & =35 \pm 10 \\
\omega_{d 0} & =(3900 \pm 200) \mathrm{Hz} \\
\tau_{d} & =(600 \pm 300) \mathrm{ms}
\end{aligned}
$$

We note immediately that the value of $\omega_{d 0}$ obtained here is consistent with that found in earlier equilibrium studies [13], noting that in that reference, $M_{2}=A \omega_{d 0}^{2}$, where in equilibrium, $A=4 / 45$. By contrast the values of $Z$ and $\tau_{d}$ differ somewhat from values obtained using deuterium $\operatorname{NMR}\left(Z \sim 49, \tau_{d}=\right.$ $210 \mathrm{~ms}$ ) [12]. This is perhaps not surprising given the very different nature of the averaging processes relevant to deuterium NMR (where the probe molecule motionally averages the segment ensemble) and proton NMR (in which a sum of signals arising from different segments is averaged). We note that the sample has finite polydispersity $\left(\frac{M_{w}}{M_{n}}=1.84\right)$ and no distribution of molecular weights has been allowed for in our simulation, nor was it considered in the earlier deuterium NMR work.

With our best-fit values, the signals generated for the horizontal Couette cell experiment (i.e., using the $h_{X}$ expression in the $A B(\gamma)$ function) are shown in fig.(4.9) below for shear rates varying between 0 and $100 \mathrm{~s}^{-1}$. The curve corresponding to a shear rate of $100 \mathrm{~s}^{-1}$ is essentially the 'infinite' shear case: increasing shear does not modify the curve further.

\subsubsection{Differences between the velocity and velocity gra- dient scenarios}

To the eye, the experimental data recorded in prior work by Cormier indicate that the shear-rate dependence of the transverse relaxation in the two cases we have looked at $(X \equiv z$ and $Y \equiv z)$ is extraordinarily similar. We may take a step back from numerical calculation and ask whether this is reasonable or not, expected or surprising, through some limiting case analysis.

Equation 4.30 and equation 4.32 are the keys in differentiating the shear-ratedependent behaviour of the distribution of unit vectors indicating the direction of the polymer tube in the tow cases, and it can be seen that when $\gamma$ is set to 
zero, both $h_{X}$ and $h_{Y}$ evaluate to $\cos ^{2} \theta$ for all $\theta$ and $\phi$ over the surface of the unit sphere. This result is in no way surprising, as the polymer undergoing no shear whatever is expected to be isotropic in tube director distribution, and no discrepancy in behaviour in the two directions can occur.

Moving then to the case where the shear applied is effectively infinite, differences can be made out. The function $h_{X}$ to be spherically averaged in this case tends to the asymptotic value of 1 , no matter what $\theta$ and $\phi$ are, while when the $Y \equiv z$ geometry is used, $h_{Y}$ tends to zero. These values directly impact the calculation of $A$ and $B$ in eqns. 4.26 and 4.27; the relaxation consequently calculated via eqn. 4.28 is clearly going to differ between the two cases. Notably, $A-B$ will be zero for both cases at infinite shear, leaving only a $B$ dependence in the relaxation function. $B$ is equal to $\frac{4}{9}$ in the $h_{X}$ case, and only $\frac{1}{9}$ for $h_{Y}$. This factor of four in an effectively scaled $\omega_{d}^{2}$ in eqn. 4.28 leads to a characteristic relaxation time difference of a factor of two. We now must decide whether this difference can be rationalised by known modifications to the Doi-Edwards theory, as it is important to retain in mind that, the experimental data (and our target data set) appear to be qualitatively the same, or at least similar.

One known modification to the basic Doi-Edwards model of polymer relaxation is "convected constraint release (CCR)". It involves incorporating the dissipation of the constrictions around which the mean-field polymer tube is formed, and lead to an decrease in the terminal relaxation time. A second demonstrated (by Cormier) implication of this modification is that the "extinction angle" of the system is non-zero as shear rate increases (this is the angle that the longest axis of the deformed spheroid of the polymer shape makes with the velocity axis in the steady-state competition between strain and relaxation), whereas it is seen to be zero without CCR. By not dropping to zero, this extinction angle behaviour implies that the polymer can never be "totally" aligned at arbitrarily high shear rates, and that the difference in the transverse relaxation for the two cases will not be as large as one might otherwise have calculated. This is the most promising possible mechanism to enable the calculated magnetic resonance relaxation rates to merge at high shear rates. 


\subsubsection{Discussion}

The Doi-Edwards model of polymer melts (being fluids of polymeric molecules which are packed as densely as allowed through internal motions yet retaining freedom to diffuse) is such that, as seen by a representative polymer, all the strands of polymer in the material temporarily form a maze of restrictions that our representative cannot slice through as it evolves in conformation. Of course, as the state of the background strands evolve themselves, each point in the grid of restrictions may fade out and new ones come into existence in other places. This particular process occurs on the largest timescale important in the description of polymer melts, known as the tube disengagement time, $\tau_{d}$.

Relatively slow motions of the centre of mass of the polymer determine the longest time over which we expect a polymer undergoing one-dimensional, curvilinear diffusion to have a remote possibilty of return-to-origin, in which a proton bound to its polymer and located in a particular tube segment may be found in that precise tube segment a time $t$ later. This return-to-origin origin probability is of vital importance to the conservation of correlation in the residual dipolar interaction generated by incomplete directional averaging of the through-space internuclear homonuclear dipole interaction with the many other protons surrounding our test nucleus.

Following the observation of NMR transverse relaxation time $\left(T_{2}\right)$ variation with shear rate, which saw the effects of shear induced alignment in a $494 \mathrm{kDa}$ melt of poly(dimethylsiloxane) [3], using it as a real example on which to tune parameters, a numerical simulation based on the simplified approach to nuclear spin correlation analysis of Ball, Callaghan and Samulski [2] was created. By incrementing this approach to include the angular distribution of tube segments following a standard affine deformation matrix approach common in rheology and Rheo-NMR, the change to the line shape of the NMR signal has been found to be of use in quantifying alignment of tube segments.

Our simulated variation in NMR signals with shear broadly match the timedependence of the experimental echo decays, in particular dependence of $T_{2}$ on shear rate. A fit to the shear-dependent $T_{2}$ data available yielded the values $\tau_{d}=600 \mathrm{~ms}, Z=35$ and $\omega_{d 0}=3900 \mathrm{~Hz}$. The $Z$ and $\tau_{d}$ values are consistent with earlier measurements made using deuterium NMR under shear, while the 
fitted value for $\omega_{d 0}$ is consistent with proton dipolar relaxation measurements under zero shear. Future work incorporating convected constraint release may aid in refining the tunable parameters in the $X \equiv z$ case, and enable the $Y \equiv z$ case to become qualitatively accurate. 


\section{Bibliography}

[1] AI Nakatani, MD Poliks, and ET Samulski. NMR investigation of chain deformation in sheared polymer fluids. Macromolecules, 23(10):2686-2692, MAY 141990.

[2] RC Ball, PT Callaghan, and ET Samulski. A simplified approach to the interpretation of nuclear spin correlations in entangled polymeric liquids. J. Chem. Phys., 106(17):7352-7361, MAY 11997.

[3] BS Douglass, RJ Cormier, and PT Callaghan. Shear deformation of polymer melt observed via proton NMR: Theory and experiment. Physical Review E, 75(4, Part 1):041802, APR 2007.

[4] M Doi and SF Edwards. The Theory of Polymer Dynamics. Oxford University Press, 1986.

[5] A Abragam. The Principles of Nuclear Magnetism. Clarendon Press, Oxford, 1961.

[6] B Deloche and ET Samulski. Short-range nematic-like orientational order in strained elastomers - a deuterium magnetic-resonance study. Macromolecules, 14(3):575-581, 1981.

[7] PW Anderson and PR Weiss. Exchange narrowing in paramagnetic resonance. Reviews of Modern Physics, 25(1):269-276, 1953.

[8] PG DeGennes. Reptation of a polymer chain in presence of fixed obstacles. J. Chem. Phys., 55(2):572, 1971.

[9] JD Ferry. Viscoelastic Properties of Polymers. Clarendon, Oxford, 1961. 
[10] ML Kilfoil and PT Callaghan. NMR measurement of the alignment tensor for a polymer melt under strong shearing flow. Macromolecules, 33(18):6828-6833, SEP 52000.

[11] JM Atkin, RJ Cormier, and PT Callaghan. Time-dependence of nuclear magnetic resonance quadrupole interactions for polymers under shear. $J$. Magn. Reson., 172(1):91-97, JAN 2005.

[12] RJ Cormier and PT Callaghan. Molecular weight dependence of segmental alignment in a sheared polymer melt: A deuterium nuclear magnetic resonance investigation. J. Chem. Phys., 116(22):10020-10029, JUN 82002.

[13] PT Callaghan and ET Samulski. The molecular weight dependence of nuclear spin correlations in entangled polymeric liquids. Macromolecules, 31(11):3693-3705, JUN 21998. 


\section{Chapter 5}

\section{Complex Fluids in Shear Flow}

\subsection{Overview}

Simple fluids under steady-state shear exhibit a velocity gradient, and the magnitude of the continuous force needed to be applied to achieve this state is proportional to the material's viscosity. However, with more complex fluids whose components can be characterised by more than one lengthscale (and correspondingly more than one timescale given the relevant dynamics of the components) and as such, regimes of behaviour are delineated by the critical rates of external deformation which correspond to these.

A single-valued velocity gradient across a sample might be expected to have some aligning influence on structures within the fluid, to a degree determined by the interplay of the absolute strain rate and any possible relaxation mechanisms the structures may have available to them. The magnetic resonance response of deuterons located in an anisotropic global distribution of carbon-deuteron bonds (whether incorporated in the main structural molecules or in some small probe molecule) is well known to split the Zeeman deuteron spectral line; the separation of the split peaks is an indicator of the degree of alignment. Magnetic resonance velocimetry is another well-established field, and can be used in conjunction with alignment-visualisation experiments, along with molecular diffusion measurements in the combination we have seen previously: Rheo-NMR.

Using Rheo-NMR to investigate truly complex fluids in this chapter means using all the available Rheo-NMR techniques to correlate velocity fields, local 
diffusion coefficients and local order parameters to aid in the investigation of worm-like micellar structures, how they form, and what their response to shear is. The most fascinating and least fundamentally understood phenomenon that worm-like micelles demonstrate is that of shear-banding, a state of affairs in which the sample is subjected to a uniform (or nearly uniform) shear stress, and in which two (or more) contiguous regions of differing shear rate are seen, while maintaining the average shear rate determined by the geometry.

Shear-banding is only a relatively recently known phenomenon, and is now established as being caused by non-monotonicity in the stress-strain relationship. In the first instance it may perhaps be understandable if shear-banding arises purely through some structural change which effectively creates two separate, de-mixed sub-samples. For some time the question of whether a shear band is in fact a birefringent band [1] has been debated. Magnetic resonance has long been held as demonstrating correlation between birefringence and an order parameter in the alignment of carbon-deuteron bonds within molecules, and much of the work presented in this chapter aims to investigate the validity of certain statements in this respect.

\subsection{Apparatus}

\subsubsection{The Rheometer}

As the basis for investigations into the correlation of various mechanical and pseudo-optical properties of materials under deformational conditions, basic rheological measurements guide us more easily towards materials that are of interest when they have the right composition, concentration and temperature. We have seen what macroscopically observable phenomena these "interesting" materials might demonstrate to qualify as 'complex' already in Chapter 2, namely shear thinning, shear thickening, and shear banding, and all these properties are easily observed in the rheometer as a codependence of stress and strain. Our rheometer used to do this is a stress-controlled TA Instruments AR2000. This instrument can also be operated in a strain-controlled manner via an internal feedback loop. Both modes are used to verify the validity of obtained data and to extend the angular frequency and shear rate ranges over which data are 
acquired. In particular, creep measurments (constant applied stress) is used in place of extremely low angular frequencies. Various geometries are available for this system, such as the Couette geometry and the cone-and-plate geometry.

Several logistical constraints limit the range of frequency and shear rates studied. Two primary considerations are the minimum measurable torque, which restricts measurements at low frequency and low shear rate, and sample ejection and/or inertial effects at high shear rates which can be problematic, especially in the more "open" (less contained) geometries such as that of the cone-and-plate. The shear-rate range covered in this work is from $0.01 \mathrm{~s}^{-1} \mathrm{up}$ to a maximum of $10 \mathrm{~s}^{-1}$.

Temperature is controlled via the flow of water past a Peltier element located at the base of the rheometer cell used. A Type $\mathrm{T}$ thermocouple in thermal contact with the plate surface is also installed to calibrate with the NMR experimental temperature, as described in the next section.

\subsubsection{Rheo-NMR}

Magnetic resonance measurements reported here are made on a Bruker 9.4 Tesla, wide-bore superconducting magnet, being controlled by a Bruker Avance $400 \mathrm{MHz}$ spectrometer. With our configuration, protons precess about this field at a nominal $400.130 \mathrm{MHz}$ (disregarding small changes possibly made by the user); deuterons precess at $61.422 \mathrm{MHz}$.

For all NMR experiments specifically aimed at investigating the response of materials to shear in this research, the Couette geometry (see section 2.3.7) is exclusively used. Comprising two concentric cylinders, with the sample located in the intervening gap, the sample can be sheared through rotation of the inner cylinder. In the limit of small gap spacing relative to cell radius, this approximates infinite planes sheared relative to each other. The maximum shear rate in the cell gap, seen at the inner wall of the gap, is given by the linear speed of the inner cylinder wall divided by the gap spacing. Experiments reported here predominantly use two concentric glass NMR tubes - a $10.0 \mathrm{~mm}$ OD, $9.0 \mathrm{~mm}$ ID tube enclosing a $7.5 \mathrm{~mm}$ OD tube - separated by Teflon spacers to maintain a fixed gap between the tubes. When imaging the velocity of samples in the gap, vibration and non-concentricity of the tubes become a critical issue. Thus, we 
use spacers near the top and in the middle of the tube gap, and ensure that the outer tube is held rigidly in the r.f. coil using Teflon spacers, o-rings, and often a thin layer of Teflon tape wound around the outer tube.

Other construction methods may be utilised: amongst these the fabrication of cells made of concentric PEEK (polyetheretherketone) tubes is most popular for us, with the outer surface of the inner cylinder being knurled or hatched in an effort to reduce slippage at the shearing plane.

\subsection{Worm-like micelles}

Worm-like micelles are one of many self-assembled micellar morphologies available to amphiphilic surfactants, and are long, thread-like agglomerates of surfactant molecules in solution [2-4], forming at concentrations above the critical micelle concentration (cmc). That they form over other micellar forms such as spherical or cylindrical micelles, or other complicated assemblies such as lamellar and sponge-like phases, depends on the details of the surface curvature and the associated free energy [5-7]. Those systems of surfactants that form wormlike micelles require a surface free energy condition which favours cylindrical curvature over other possible states. A wide range of worm-like micelle systems require the presence of a cosurfactant or counter-ion: canonical examples are the surfactant micellar systems CTAB (cetyltrimethylammonium bromide) in water [8] and $\mathrm{CPyCl} / \mathrm{NaSal}$ (cetylpyridinium chloride/sodium salicylate) in water $[9,10]$.

The region of declining stress with increasing shear rate in the flow curve of such materials is inherently unstable and leads to coexistence of flow of material following separate stress branches, in a proportion satisfying the overall rate of strain condition. Shear banding in the stress plateau region has been convincingly demonstrated by techniques as diverse as NMR velocimetry [1113], LASER Doppler velocimetry (LDV) [14], dynamic light scattering [15-18] and ultrasonic velocimetry [19-21]. That shear banding, in systems close to the isotropic-to-nematic (I-N) phase transition, may be associated with coexisting states of different orientational order, is suggested by birefringence [8, 22, 23], NMR spectroscopy [24] and small-angle neutron scattering (SANS) [25]. In many of these studies, shear-banded flow has been associated with shear-induced 
phase transitions, wall slip, and shear stress fluctuations.

\subsection{Rheology of worm-like micelles}

Surfactant/cosurfactant systems exhibiting worm-like micellar behaviour have three principal rheological characteristics. Firstly, there exists a region of the phase diagram (in which the proportions of surfactant and cosurfactant are adjusted), where a significant increase in zero shear (or low shear) viscosity occurs. This viscous phase is generally associated with the formation of long thread-like micelles whose dynamics are dominated by the interplay between reptation and dissociation-recombination; these are worm-like micelles in the semi-dilute regime. Secondly, the linear rheology of such semi-dilute worm-like micelles [26] is characteristic of a Maxwell fluid, exhibiting a semi-circular ColeCole plot. At high frequencies, deviation from simple Maxwell behaviour is associated with dissociation-recombination processes. A characteristic feature of worm-like micellar non-linear rheology is the presence of a plateau on the flow curve, generally associated with the sub-division of the material into coexisting low and high shear rate phases, a manifestation of shear banding. Furthermore, this shear-banded state is often associated with strong fluctuations. Stress oscillations have been found for a variety of micelle systems [1, 24, 25, 27-33] and are sometimes associated with the growth and destruction of the shear-induced structures, in shear thickening systems. Stress fluctuations may be purely periodical or suggestive of chaos [30-32]. Recently Fielding and Olmsted have shown that such instability may be a consequence either of coupling of the hydrodynamics to a strain-rate dependent micellar variable, such as micelle length or concentration $[34,35]$. Experiments have been unable to elucidate the origin of instabilities, although in velocimetry studies, fluctuating wall slip has been shown to play a significant role $[21,31,32]$.

In the following section, we first present the phenomenological rheology data on Pluronic P105 / phenylethanol worm-like micelles exhibiting some unusual behaviour, including two maxima in the temperature dependence of apparent viscosity at high shear rates. We then show how rheo-NMR, specifically ${ }^{2} \mathrm{H}$ NMR splittings indicative of alignment, can be used to help understand the rheological observations. 


\subsection{Pluronic P105}

We follow the strategy of comparing rheometric and rheo-NMR properties of a particular Pluronic/phenylethanol phase, specifically one which has been selected due to its exhibition of a significantly enhanced low shear viscosity, and because neutron diffraction data is consistent with scattering from threadlike objects. The existence of this viscous phase is highly temperature dependent, so that temperature is an important parameter of the study. We examine both the linear viscoelastic behaviour, as well as the flow curve, taking care to elucidate time dependence in the stress. This is of particular importance in the present system because of the existence of slow (e.g. $\sim 100-1000 \mathrm{~s}$ ) time scales in the rheological behaviour. Finding that fluctuations play a role in this system, and that dynamics depend sensitively on both shear rate and temperature, we focus on shear rate and temperature as the primary variables.

\subsubsection{Sample details}

The nonionic worm-like micelle solution used in this study is composed of a solution of $5 \% \mathrm{w} / \mathrm{w}$ of the BASF Pluronic P105 in $\mathrm{H}_{2} \mathrm{O}$, to which, per $5 \mathrm{~mL}$ of Pluronic solution, $0.213 \mathrm{~g}$ of 1 -phenylethanol- $d_{5}$ (Cambridge Isotopes) was added.

P105 is a tiblock copolymer comprised of polyethylene oxide "PEO" (A) and polypropylene oxide "PPO" (B) in an A-B-A configuration. These groups normally exhibit very little difference in chemical functionality, but the slight difference in their affinities for water drives their micellar formation. This structure formation is enhanced, and the exact curvature of the structure determined, by the presence of the phenylethanol molecules. The preferential location of these molecules is to be determined, but initial thinking is that, as so-called hydrophobic alcohols, will act as a core-stabiliser for cylindrical micelles. In particular, the sample on hand has deuteron substitution only on the aromatic ring portion of the molecule. This anisotropic component of the molecule is the intended signalling mechanism of micellar alignment. 


\subsubsection{Rheometry}

Bearing in mind that Rheo-NMR experiments are strictly strain-rate controlled (no torques are measured in the use of a Rheo-NMR drive shaft), most rheometry in conventional rheometers is also carried out in a strain-controlled mode (that is, choose a strain rate, and measure the stress response of the sample). Stresscontrolled methods are also briefly used (in which specified torques are applied to the surface of a sample, and the correspondingly allowed strain is measured).

To first come to terms with the phase behaviour of the P105 solution, both linear and non-linear rheological measurements were performed. In particular these showed that there are indeed conditions in which the sample will show stress plateau characteristics, and that shear-banding would occur in a very select temperature range.

Fig.5.1(a) shows the temperature dependence of the viscosity of the Pluronic micellar solution, at a range of low shear rates. As the low-shear-rate viscosity is amplified by a factor of $\sim 10^{4}$, we can only deduce that we have formed worms long enough to entangle at this concentration. Remarkably, the region of high viscosity is rather small, covering a temperature range of only 15 degrees (from $\sim 290 \mathrm{~K}$ to $\sim 305 \mathrm{~K}$ ), although this is extended a small amount with increasing shear rate. At shear rates $>0.1 \mathrm{~s}^{-1}$, a local minimum in the temperature dependence of viscosity is seen, at $294 \mathrm{~K}$, in the midst of the worm-like micelle temperature regime! This might arise from our worms becoming sufficiently long to either form a nematic phase at high rates or break at high rates. We find that this local minimum is mirrored in shear-dependent NMR spectroscopic properties of the micelle solution.

The linear viscoelastic response of the micelle solution is shown in Fig.5.1(b). These data are also indicative of a highly entangled solution of worm-like micelles. At low frequencies, behaviour typical of a Maxwell fluid is found, while at higher frequencies strong deviations from Maxwell behaviour are observed. Note, however, that the angular frequency $\sim 5 \times 10^{-3} \mathrm{~s}^{-1}$ at which this break from Maxwellian behaviour is found is relatively low when compared with other worm-like micelle solutions, and may thus be consistent with a very slow micellar dissociation rate. This immediately raises the question of thixotropy and the need to allow for time dependence in rheological measurements of the 
nonlinear viscosity. Thus, we show in Fig.5.1(c) time dependent stress measurements over a range of shear rates. Here, characteristic relaxation times of up to $\sim 500 \mathrm{~s}$ are found before a stress plateau is reached, and consequently we take special care in establishing the flow curve for this material. Fig.5.1(d) shows a flow curve composed of a strain-rate-controlled measurement, in addition to a stress-controlled creep measurement made to measure sufficiently low strain rates to demonstrate the Newtonian region of the material. Of particular note is the coincidence of the lowest shear rate showing temporal stress fluctuations at $1.58 \mathrm{~s}^{-1}$ (Fig.5.1(c)), and the shift of the flow curve into the high-shear-rate branch (Fig.5.1(d)).

\subsection{3 ${ }^{1} \mathrm{H}$ spectroscopy and diffusometry}

An important step in the detective process of studying worm-like micelle solutions of P105 is the identification of NMR spectral lines. Besides a very good guess at identifying individual spectral lines being possible through standard chemical shift-based positions of functional groups, diffusometry can give insight by demonstrating whether certain functional groups are diffusing through the material at the expected rate, usually based on the mass of the structure diffusing. Fig.5.2 shows the $400 \mathrm{MHz}$ proton NMR spectra obtained from the Pluronic worm-like micelle system in $\mathrm{D}_{2} \mathrm{O}$ at three different temperatures, $282 \mathrm{~K}$, $293 \mathrm{~K}$ and $307 \mathrm{~K}$. Note the temperature dependence of the HDO/OH peak in the vicinity of $4.7 \mathrm{ppm}$, superposed on a temperature independent $\mathrm{CH}$ group from the phenylethanol. The upper part of the figure shows the correspondence between chemical species and the diffusion coefficient which each experiences, obtained by acquiring spectra as a function of field gradient strength in a PGSE experiment. The most rapid decay in intensity corresponds to the fastest diffusing molecules. The HDO peak exhibits the fastest molecular diffusion rate, while the phenyl alcohol aromatic ring and methyl groups are also easily identified at $-3.625 \mathrm{ppm}$ and $+2.625 \mathrm{ppm}$ respectively, with respect to the Pluronic $\mathrm{O}-\mathrm{CH}_{2}$ line. At $0 \mathrm{ppm}$ and $+2.625 \mathrm{ppm}$ we observe peaks associated with extremely small diffusion rates, which we assign respectively to the polyethylene oxide and polypropylene oxide $\mathrm{CH}_{2}$ and polypropylene oxide methyl groups on the Pluronic surfactant chains of the micelles. 
(a)

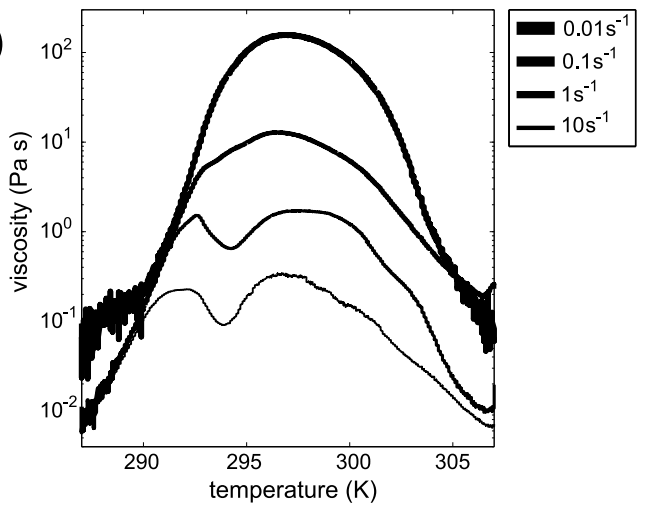

(b)

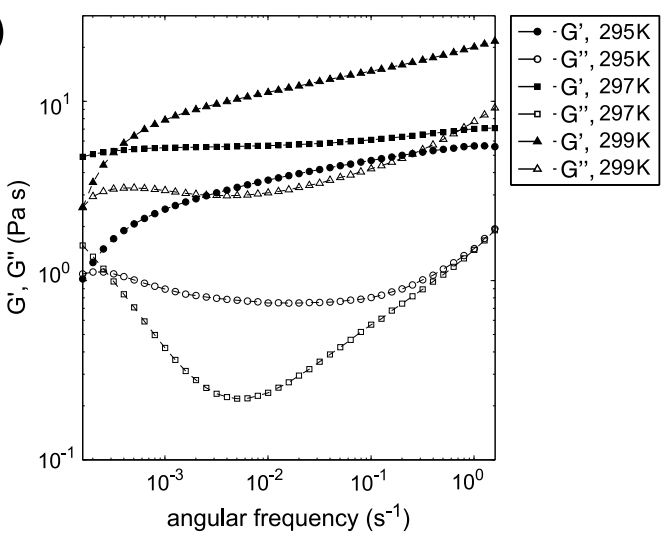

(c)

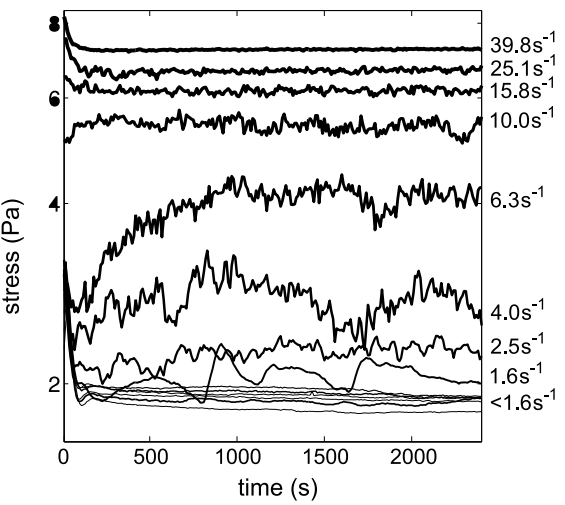

(d)

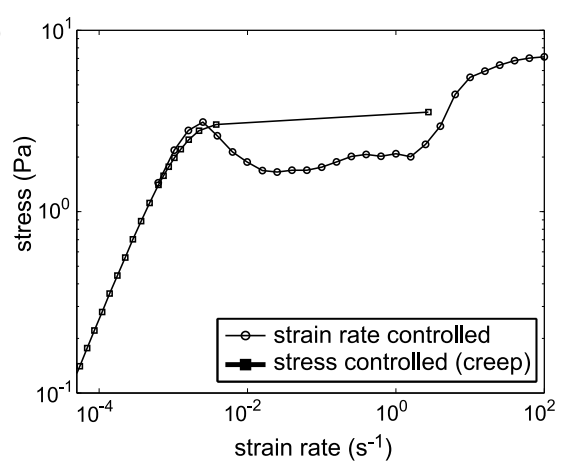

Figure 5.1: (a) The shear viscosity of the Pluronic worm-like micelle solution, obtained at various shear rates, including close to zero shear (b) The linear rheology of the P105 solution shows a crossover of $G^{\prime}$ and $G^{\prime \prime}$ at $1.6 \times 10^{-4} \mathrm{~s}^{-1}$ or lower, indicating a terminal relaxation time of $\sim 1000 \mathrm{~s}$. The upturn of $G^{\prime \prime}$ at $297 \mathrm{~K}$ occurs at $\sim 5 \times 10^{-3} \mathrm{~s}^{-1}$, potentially indicating a breakage / recombination time for the micelle of around $200 \mathrm{~s}$, and hence (via eqn. 2.32) a reptation time of $\sim 5000 \mathrm{~s}$ (c) Transient stress response to shear startup, at $297 \mathrm{~K}$. Stress fluctuations are visible between 1.6 and $6.3 \mathrm{~s}^{-1}(\mathrm{~d})$ The nonlinear rheological flow curve of the material at $297 \mathrm{~K}$, using both stress- and strain-controlled modes of rheometry. 

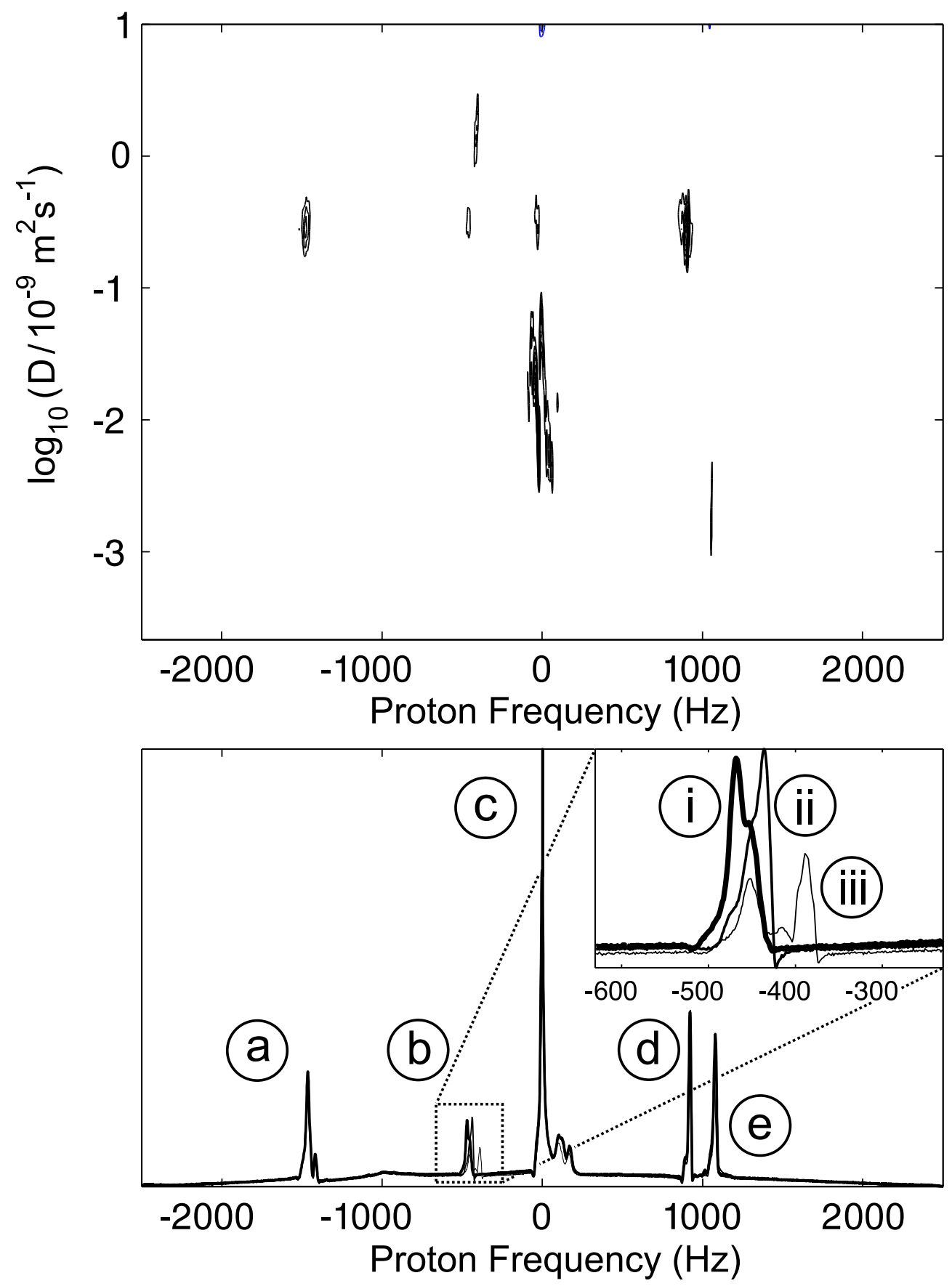

Figure 5.2: The proton NMR spectrum of the Pluronic worm-like micelle solution (below) and the DOSY spectrum resolving the diffusion properties of each spectral line (above). These lead to the following peak assignments: (a) $\mathrm{C}_{6} \mathrm{H}_{5}$ (phenylethanol) (b) $\mathrm{CH}$ (phenylethanol) in addition to $\mathrm{HDO}$ at three temperatures (i) $282 \mathrm{~K}$, (ii) $293 \mathrm{~K}$, and (iii) $307 \mathrm{~K}$ (c) $\mathrm{O}-\mathrm{CH}_{2}$ (PEO, PPO) (d) $\mathrm{CH}_{3}$ (phenylethanol) (e) $\mathrm{CH}_{3}$ (PPO). 
When we inspect the deuterium NMR spectrum for samples of the Pluronic worm-like micelle system in $\mathrm{H}_{2} \mathrm{O}$, we observe a weak signal at $4.6 \mathrm{ppm}$ upfield of the aromatic deuterons of the phenylethanol. We thus attribute this weak signal to HDO deuterons at natural abundance, an identification confirmed by the intensity ratio of this peak to that of the aromatic deuterons. Second, the temperature dependence of the HDO peak provides a useful internal calibration for temperature.

\subsubsection{Deuteron NMR spectroscopy}

Our first NMR examination of shear-dependent behaviour consists of a deuterium NMR study of molecular ordering, probed via splittings of the phenylethanol deuterium peaks. Their signal results from all fluid in the gap, and thus represents the average degree of order in the cell. We show in Fig.5.3 a series of temperature and shear-rate regimes, in which a finite order parameter is demonstrated. That this is a quadrupolar interaction, was indicated by both supplementary double-quantum filter and 2D Hahn echo experiments in which the bilinear nature of the quadrupolar interaction was apparent.

The existence of two splittings is a curious aspect of this work and a feature seen through much of this study. We discuss our interpretation of this phenomenon in Section 5.5.7. Here we present the range of observed spectral behaviours. Fig.5.3 shows ${ }^{2} \mathrm{H}$ NMR spectra obtained over a range of temperatures $(279 \mathrm{~K}$ to $308 \mathrm{~K})$ and shear rates $3.1 \mathrm{~s}^{-1}$ to $157.1 \mathrm{~s}^{-1}$. These are presented as two-dimensional colour plots with the colour representing the intensity of the ${ }^{2} \mathrm{H}$ NMR peaks. As small spectral features are hard to discern on the figures, also shown are selected spectral slices at various temperatures. Some particular features of the spectra are noteworthy. First, we note that the dominant peak, centred at $0 \mathrm{~Hz}$ corresponds to the deuterated aromatic ring of the phenylethanol. At $4.6 \mathrm{ppm}$ upfield can be found a weak ${ }^{2} \mathrm{H}$ NMR peak associated with the residual natural abundance deuterons in the water solvent. Note that the frequency of this peak is strongly temperature dependent, as described in Section 5.5.3. Second, we note that the aromatic peak is split, indicating molecular ordering, over particular bands of temperature, and that the splitting is dual in nature, with the greatest peak intensity associated with a narrow split- 


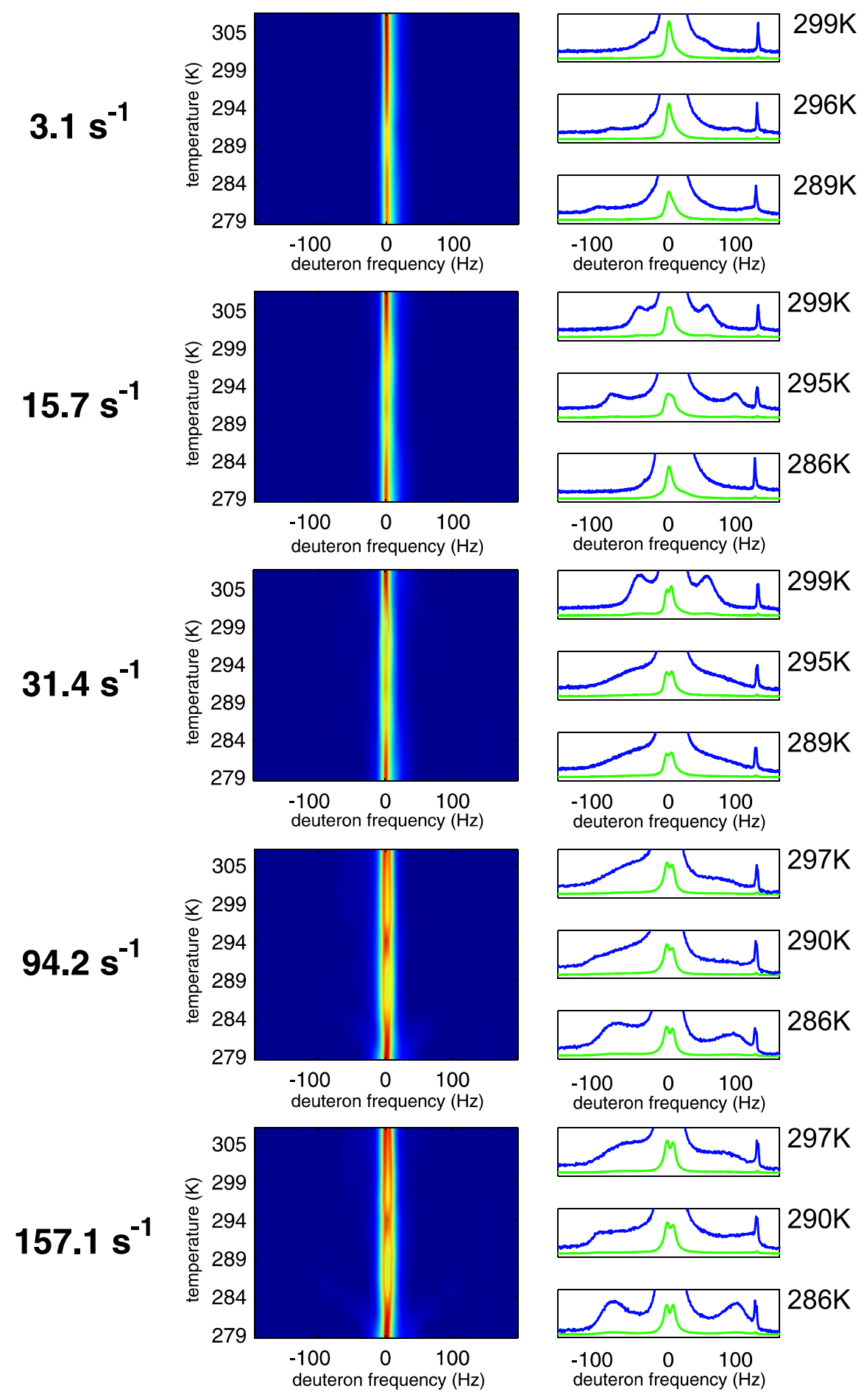

Figure 5.3: Deuterium NMR spectra as functions of temperature at five shear rates are shown in the five left panes. We show $1 \mathrm{D}$ spectra at three demonstrative temperatures for each shear rate on the right. These 1D spectra are shown at full intensity range (green), as well as blown up by a factor of 20 (blue), to show less intense features. Not shown are spectra in the vicinity of $294 \mathrm{~K}$, as a single spectral line results. 
ting of around $20 \mathrm{~Hz}$, and a much weaker component whose splitting is much larger, varying strongly with temperature to values up to $200 \mathrm{~Hz}$. This outer peak splitting is well defined at the higher end of the temperature range when the shear rate is low and even at the lowest shear rate of $3.1 \mathrm{~s}^{-1}$, but changes become well defined at lower temperatures only when the shear rate increases above $40 \mathrm{~s}^{-1}$.

In the vicinity of $294 \mathrm{~K}$, the dominant peak splitting disappears for all shear rates. This null in the dominant peak splitting is not clearly mirrored in the weak outer splitting, which instead seems to exhibit a state in which the order parameter is ill-defined. We note that a similar inflection is seen in the low shear viscosity data, also at $294 \mathrm{~K}$.

\subsubsection{Spatially resolved velocimetry}

We now turn to rheo-NMR measurements in which imaging is used to spatially localise the properties of the fluid across the gap. First we focus on NMR velocimetry. At flow startup the velocity profile exhibits a near-Newtonian profile, evolving over a period of a few minutes to a steady state in which strongly non-Newtonian behaviours are apparent. This effect is illustrated in Fig.5.4, where we show a succession of velocity profiles, separated in time by $60 \mathrm{~s}$, at an exemplary temperature of $304 \mathrm{~K}$ and shear rate of $6.2 \mathrm{~s}^{-1}$. Note that steady state behaviour in this case is achieved after a period of approximately 10 minutes, or slightly longer, which is in excellent agreement with the transient stress response for the shear rate of $6.3 \mathrm{~s}^{-1}$ shown in 5.1(c). In all subsequent data, the fluid was driven in steady shear for a time sufficient $(>10 \mathrm{~min}$ ) for the fluid velocity to reach such a steady state.

The NMR pulse sequence used here was a combination of the projection profile technique (single imaging gradient pulse in the phase direction), though with a PGSE $q$-encoding gradient wrapped around one of two slice-selective 180 degree pulses. Two slice selections were required to only receive signal from two well defined columns in the gap of the cylindrical Couette cell.

Similar to the above ${ }^{2} \mathrm{H}$ NMR measurements of molecular ordering, the fluid velocity profiles shown in figs. 5.5 and 5.6 are strongly temperature and shearrate dependent. We present, for simplicity, two canonical examples, in which 

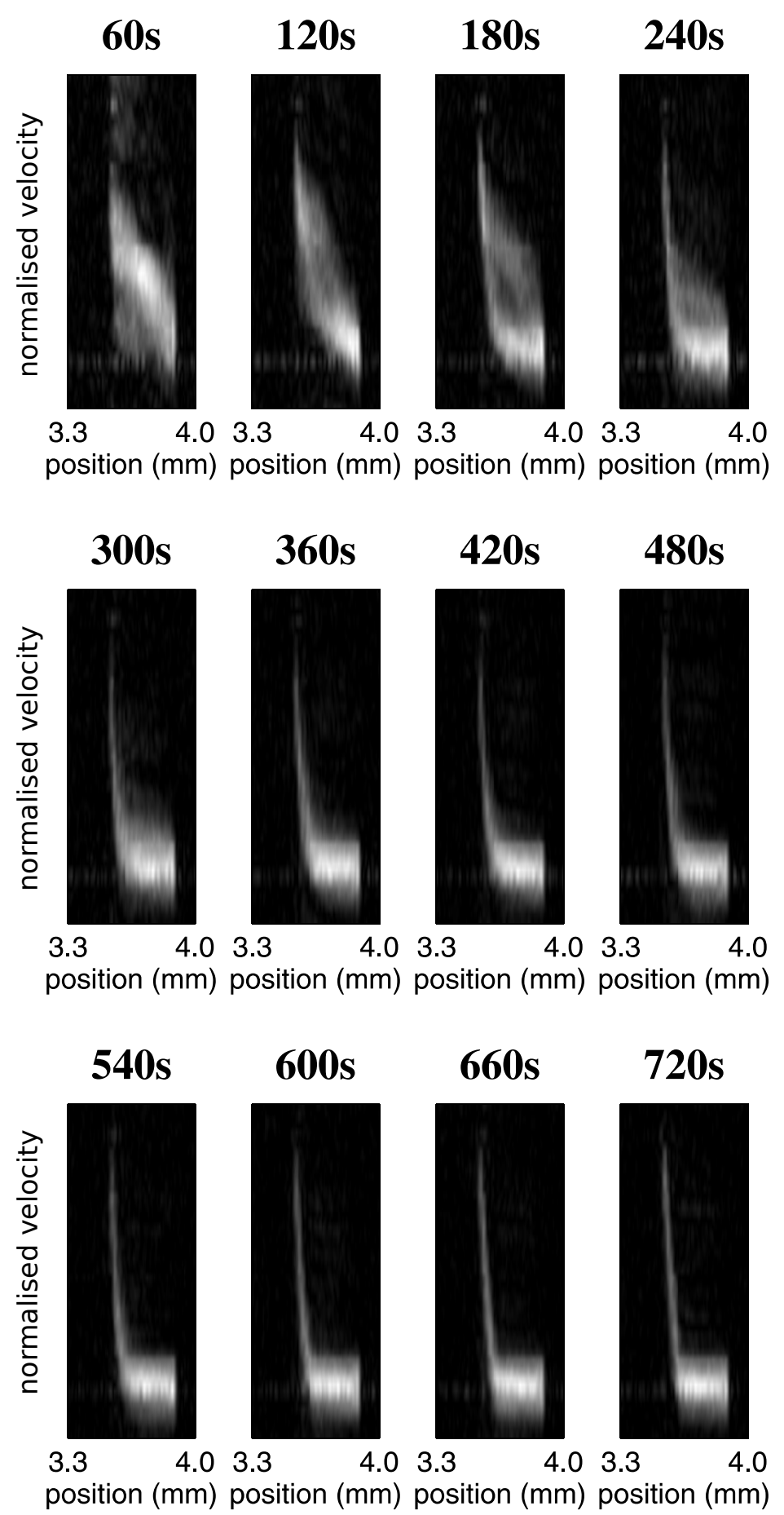

Figure 5.4: A time series of velocity maps is shown for a single shear rate, $6.2 \mathrm{~s}^{-1}$, at a single temperature, $304 \mathrm{~K}$. The times indicated are relative to the time at which the shear rate was stepped from zero, a terminal shear rate profile can be seen after $600-700 \mathrm{~s}$. 
shear rates are swept over the entire range of interest, at temperatures of $293 \mathrm{~K}$ and $304 \mathrm{~K}$. In each case we measure a pre-selected diametral slice of width $1 \mathrm{~mm}$ (along the velocity direction) and depth $5 \mathrm{~mm}$ (along the cell axis) taken across the Couette cell. The signal data are shown as two-dimensional greyscale maps in which the horizontal dimension is the radial position across the Couette cell, while the vertical dimension is the fluid velocity, calculated from the measured displacement over the encoding times $\Delta$ used in each case, ranging from $9 \mathrm{~ms}$ up to $48 \mathrm{~ms}$. There is an expected broadening of displacements resulting from diffusion alone, however this is not a major cause of spread in the propagator, especially at the higher shear rates. Broadening in most cases here therefore corresponds to a velocity distribution and/or fluctuations.

Fig. 5.5 shows the case of $293 \mathrm{~K}$, in which a range of different shear profile behaviours is exhibited. At the lowest shear rate, $3.1 \mathrm{~s}^{-1}$, shear banded flow is apparent. At higher rates, in the range 6.3 to $62.8 \mathrm{~s}^{-1}$, fluctuations are seen, and banding consists of a high shear region both at the inner wall and the outer

wall of the gap. Above $62.8 \mathrm{~s}^{-1}$, the banding disappears and the flow returns to near-Newtonian character, although fluctuations still play a role. Fig. 5.6 shows shear rate profiles for the case of $304 \mathrm{~K}$. Here, a well-defined shear band is demonstrated at low shear rates (up to $12.6 \mathrm{~s}^{-1}$ ), then a transition through unstable flow to near-Newtonian behaviour above $25 \mathrm{~s}^{-1}$. The inward curvature at highest shear rates results from azimuthal movement of the fluid near the inner wall, over the times taken (between 1.6 and $5 \mathrm{~ms}$ ) to encode for transverse spatial position.

\subsubsection{Spatially resolved spectroscopy}

We now turn our attention to the spatial localisation of molecular ordering. In this case we use the Hankel transformation method, described in Chapter 3, to produce a radial image of the ${ }^{2} \mathrm{H}$ NMR spectrum. Figs.5.7 and 5.8 compare the spectral and velocity profile images. Spectroscopic images are presented as two dimensional greyscale plots with the horizontal dimension the radial dimension and the vertical dimension the ${ }^{2} \mathrm{H}$ NMR frequency, with spectra from the radial slices nearest the inner and outer walls shown above. The results are quite remarkable and counterintuitive, given the common presumptions about shear- 

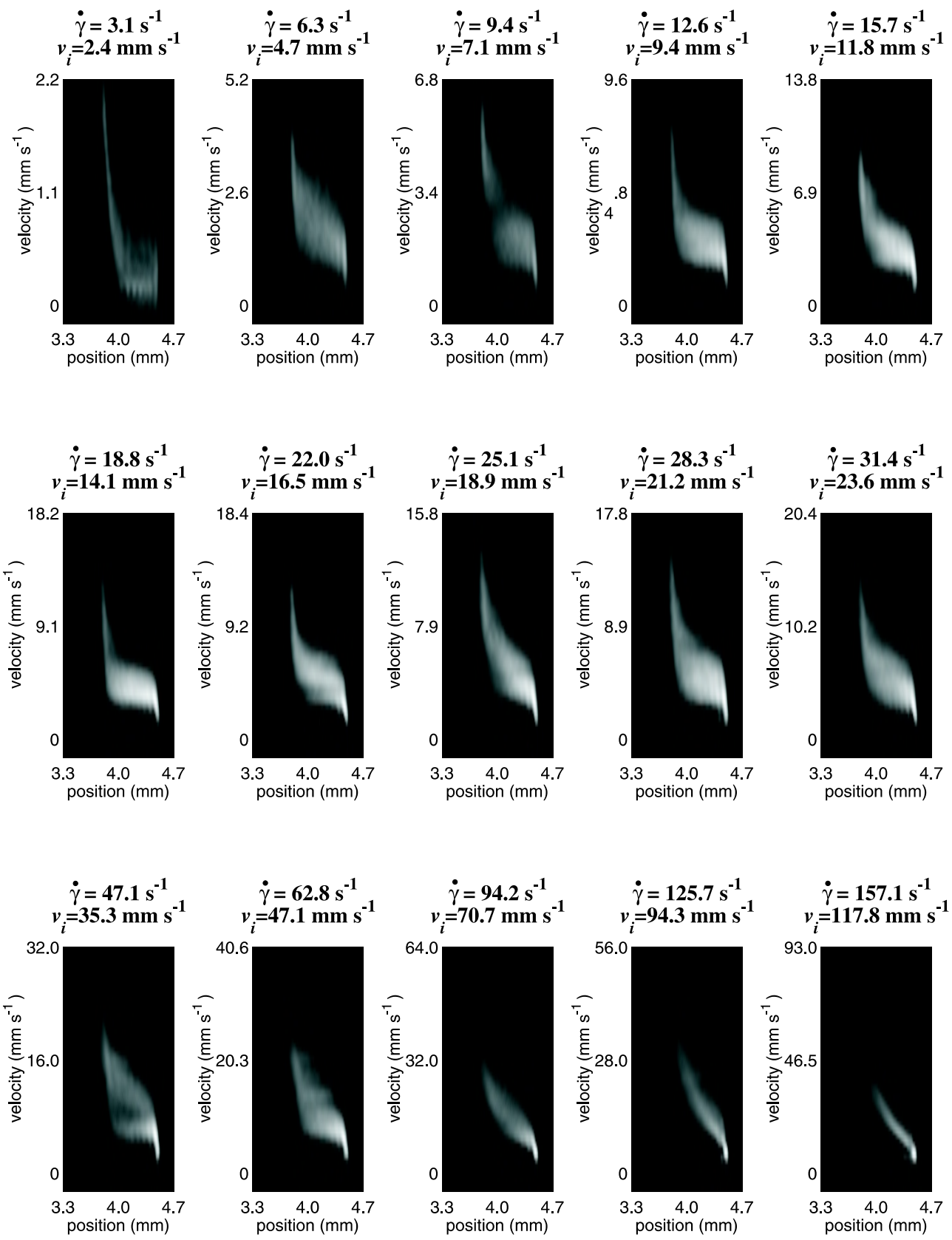

Figure 5.5: (a) The velocity profiles are shown here at a range of average shear rates at $293 \mathrm{~K}$. The inner wall velocity at corresponding shear rate is indicated. 

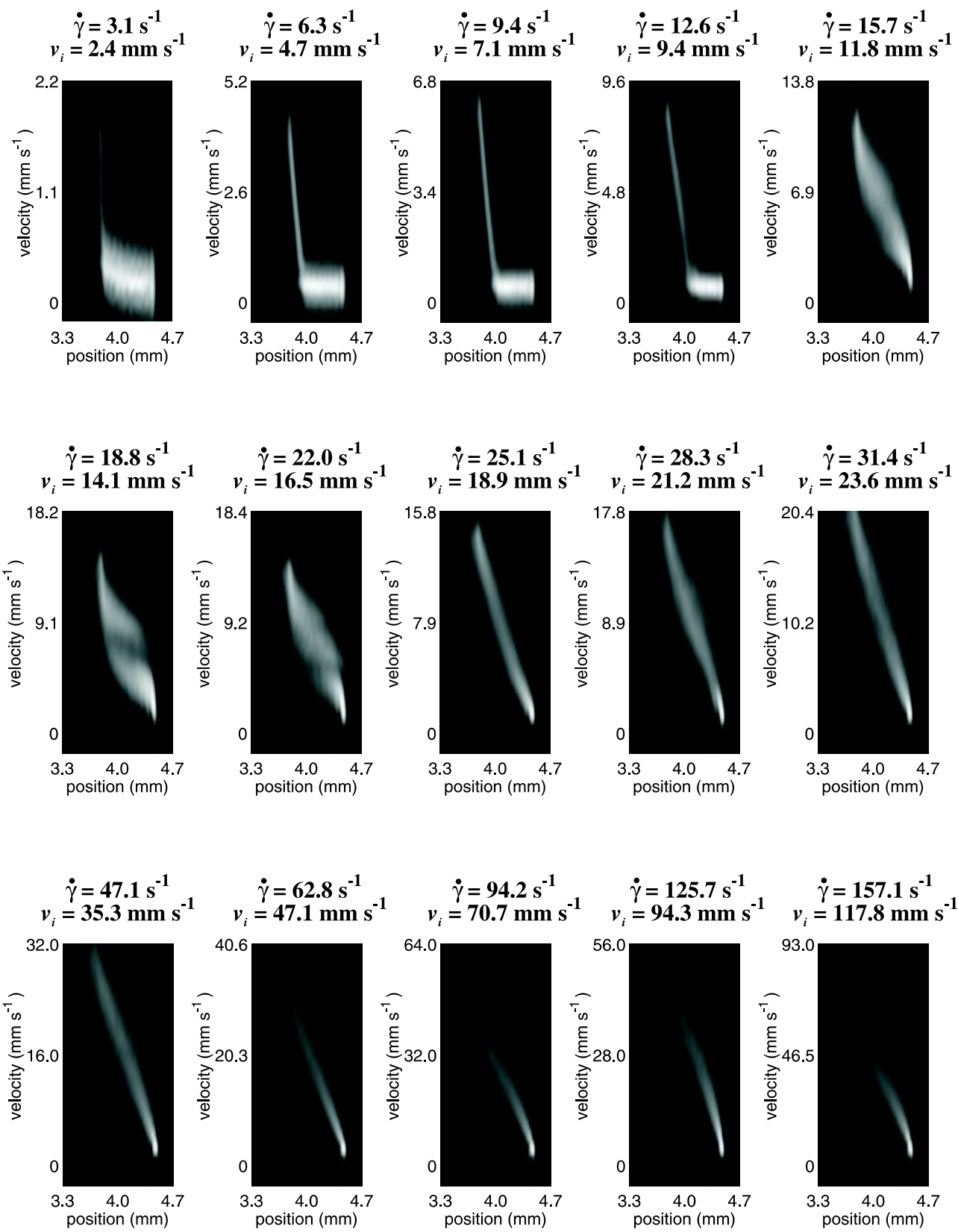

Figure 5.6: (a) The velocity profiles are shown here at a range of average shear rates at $304 \mathrm{~K}$. The inner wall velocity at corresponding shear rate is indicated. 
banded flow. Several researchers have argued that shear banding in worm-like micelle solutions is associated with nematic ordering. However the rheo-NMR experiments of Figs.5.7 and 5.8 show that such a simple correspondence does not exist.

Indeed, those cases where shear banding occurs, as evidenced by velocimetry, are associated with lack of observed molecular ordering. By contrast, where apparent Newtonian flow is present, strong ordering effects also appear, clouding any possible conclusions concerning spatial correlation between birefringence and shear rate bands.

\subsubsection{Possible models for phase behaviour}

Clearly, more than one physical feature of our micellar system is driving the observations in our array of rheo-NMR experiments. Indeed, this is precisely why the term 'complex fluid' applies to worm-like micelle solutions. In contrast to simple linear polymer solutions, they form a "living" system where these supramolecular worms can break and can recombine with each other. They might also form more complex phases such as branched micelles, vesicles, lyotropic lamellar phases, spherical micelles or some combination of these possibilities. Similar to linear polymers, worm-like micelles can entangle and can align with each other, and might form a nematic phase in a shear field. Anisotropic alignment may also act in a different way on these materials due to the different nature of stiffness along their axes. The concept of entanglement may differ substantially from that in linear, covalently bound, polymers in that when a worm tube encounters a topological constraint (another tube) it may join with it, or break, or pass through it with some characteristic timescale, possibly depending on shear rate. Our data show several phenomena that narrow the scope of possibilities for the phase behaviour of these systems.

The local minima in the temperature dependence of apparent viscosity at high rates (Fig.5.1(a)) is highly unexpected, as the worms likely grow the longest at these intermediate temperatures. With very long worms, two possible mechanisms come to mind for explaining the viscosity reduction at high shear rates. Have the worms grown long enough to flow-align and form a nematic phase at high rates? Or perhaps when long enough, they can break in shear flow to 

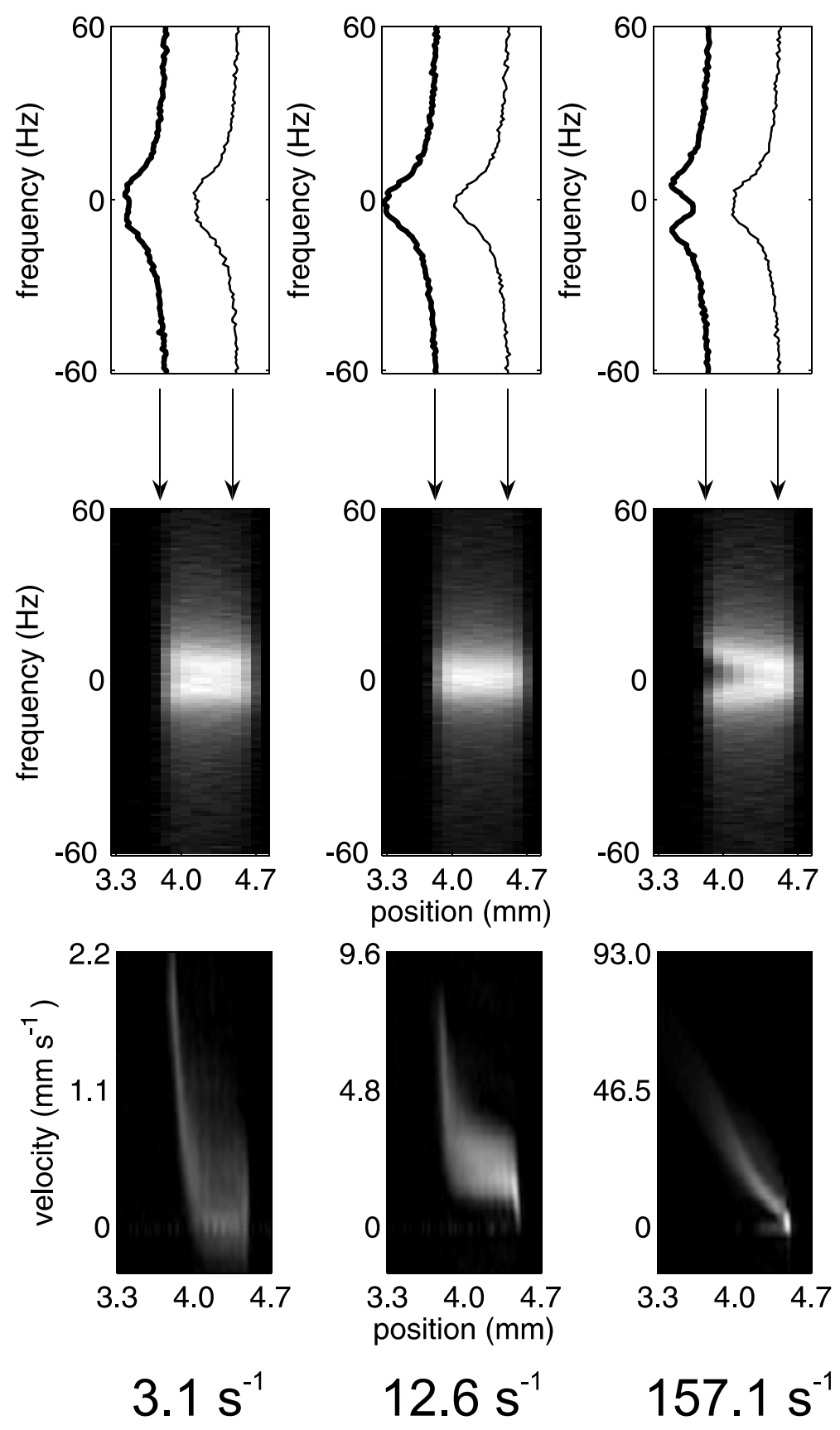

Figure 5.7: (a) Variation of the velocity profile with shear rate at $293 \mathrm{~K}$. The inner wall velocities for the gap-averaged shear rates of $3.1 \mathrm{~s}^{-1}, 12.6 \mathrm{~s}^{-1}$, $157.1 \mathrm{~s}^{-1}$ are $2.4 \mathrm{~s}^{-1}, 9.4 \mathrm{~s}^{-1}$ and $117.8 \mathrm{~s}^{-1}$ respectively. The top three panes indicate the NMR spectrum of the sample at the inner and outer walls, as obtained through the radial spectroscopic technique described. 

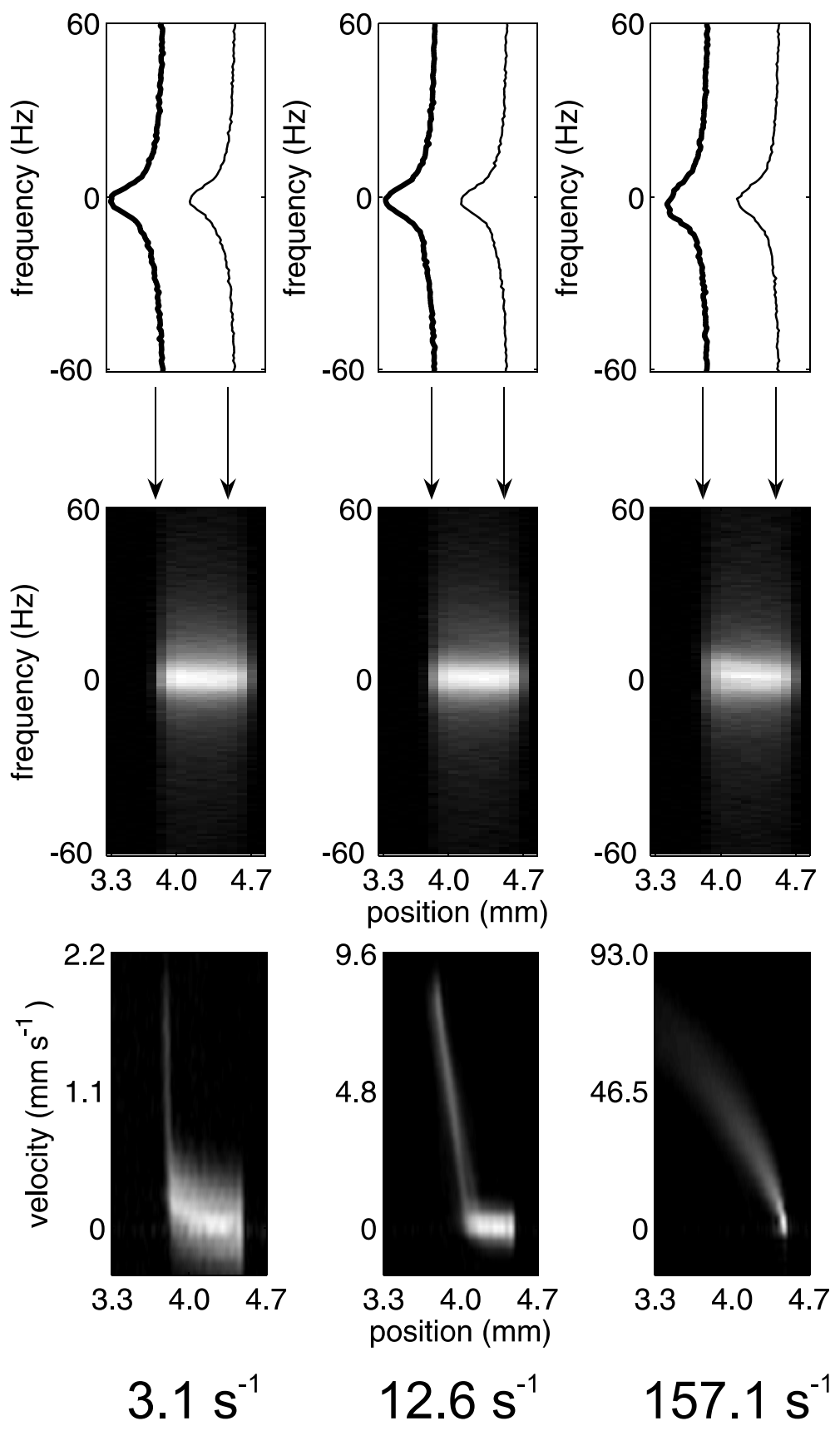

Figure 5.8: (a) Variation of the velocity profile with shear rate at $304 \mathrm{~K}$. The inner wall velocities for the gap-averaged shear rates of $3.1 \mathrm{~s}^{-1}, 12.6 \mathrm{~s}^{-1}$, $157.1 \mathrm{~s}^{-1}$ are $2.4 \mathrm{~s}^{-1}, 9.4 \mathrm{~s}^{-1}$ and $117.8 \mathrm{~s}^{-1}$ respectively. The top three panes indicate the NMR spectrum of the sample at the inner and outer walls. 
lower their viscosity. The temperature dependent ${ }^{2} \mathrm{H}$ NMR splitting in Fig.5.3 can distinguish between these two viscosity reduction mechanisms. If a nematic phase formed at $294 \mathrm{~K}$, we would expect to see an enhanced splitting. Instead the inner splitting disappears completely, suggesting that shear simply breaks the worms at high shear rates near $294 \mathrm{~K}$.

On first inspection, we supposed that the two ${ }^{2} \mathrm{H}$ NMR splittings seen in the spectra of Fig.5.3 might be due to one of the following two hypotheses:

1. the two distinct ${ }^{2} \mathrm{H}$ sites (in terms of $\mathrm{CD}$ bond angle with respect to $B_{0}$ ) on the phenylethanol molecule, if it were ordered along a well defined axis, or

2. the probe molecule could be partitioning between two parts of the worms, for example the core and corona.

On close inspection, hypothesis (1) does not fit the data in that if it were true, the two pairs of lines would have exactly the same (proportional) dependence of splitting on temperature, and their linewidths would also have the same dependence. Hypothesis (2) seems far more likely. Indeed, SANS data suggest that, while the phenylethanol prefers the core (hydrogen bonded to the ether oxygen of PPO), it also exists in the corona [36] (hydrogen bonded to the ether oxygen of PEO). The diffusion coefficient of the probe molecule (see Fig.5.2), $D \approx 3 \times 10^{-10} \mathrm{~m}^{2} \mathrm{~s}^{-1}$, means that the probe molecule diffuses approximately $\sqrt{6 D t} \approx 10 \mu \mathrm{m}$ on the timescale of our ${ }^{2} \mathrm{H}$ NMR spectroscopy experiment $(\sim$ $0.05 \mathrm{~s})$. While this is considerably larger than the core diameter $(7 \mathrm{~nm})$ of the worms [36], the diffusion of the probe in the core may be considerably more facile along the worm direction, apparently with slow exchange between core and corona states of the phenylethanol probe. We can only explain the Fig.5.3 data with the idea that the probe molecule must exist in two distinct phases or domains of the system, which are stable at least on the $\sim 0.05 \mathrm{~s}$ timescale. Furthermore, examining the Fig.5.3 data, it is clear that the relative amounts of the two phase or domain types do not change over the observed temperature range of $30 \mathrm{~K}$.

Just as interesting is the complexity in the temporal character of shear rate profiles, as revealed by the time-resolved and steady state NMR velocimetry. 
The thixotropy of this micellar system, as seen in the rheological measurements, is also apparent in the time taken to reach a steady state velocity profile, as evidenced in the data of Fig.5.4, where a time series of velocity maps for the shear rate, $6.2 \mathrm{~s}^{-1}$ at $304 \mathrm{~K}$ indicates an initially fluctuating profile, with a steady shear-banded state formed after $600 \mathrm{~s}$.

Remarkably, once this steady state is reached, there is no obvious relationship between shear banding and a state of nematic order. In Figs.5.7 and 5.8, obtained at two different temperatures of $293 \mathrm{~K}$ and $304 \mathrm{~K}$ respectively, no correspondence can be ascertained. At $293 \mathrm{~K}$ and $3.1 \mathrm{~s}^{-1}$ we see evidence of nematic order through the split deuterium spectrum in conjunction with a shear banded state, and yet there is no discontinuity of nematic order associated with the discontinuous shear rate. At $304 \mathrm{~K}$, at the same gap averaged rate of $3.1 \mathrm{~s}^{-1}$, as well as at $12.4 \mathrm{~s}^{-1}$ no nematic order is seen at all, despite manifestly shearbanded states. The greatest degree of nematic order is found at $293 \mathrm{~K}$, under conditions where no shear banding is apparent at all. This nematic order varies continuously across the gap, even though the shear rate appears homogeneous. Finally, in relation to shear rate profiles, both fluctuating and steady profiles are observed, the sweep at $301 \mathrm{~K}$ shown in Fig.5.6 exhibiting transitions from fluctuating to steady to fluctuating as the gap-averaged strain rate is gradually increased.

This bewildering array of phenomenology is more complex than is found in the classical worm-like micellar systems based on ionic amphiphiles, examples being cetylpyridinium chloride/sodium salicylate in water [9] and cetyl trimethylammonium bromide in water [24]. The most noteworthy difference is the strong temperature dependence of and temperature inflection in the viscosity at finite shear rate. Nonetheless there are some similarities in the lack of clear correspondence between shear bands and nematic ordering [24], as well as the diversity of fluctuation dynamics [32] which classical ionic surfactant micelles exhibit. We present these data here in order to illustrate the rich diversity of phenomenology that remains to be explained in the rheology of Pluronic surfactant phases. 


\subsection{Conclusions}

The rheo-NMR methods presented here combine novel spatially resolved NMR spectroscopy and velocimetry. The high resolution ${ }^{2} \mathrm{H}$ NMR spectroscopic measurements are made possible by the use of Hankel transformation methods which utilise the entire signal from the entire Couette cell annulus. It depends on the use of a deuterated probe molecule, in this case the phenylethanol needed for the micelle formation. The velocimetry data was obtained using a rapid single slice phase-encoding method which permitted velocity profiles at 1 s repetition times. The assignment of peaks in the NMR spectrum has been assisted by the use of spectrally-resolved molecular diffusion (DOSY) measurements.

The most significant findings concern:

1. A flow curve exhibiting plateau-like properties in the stress with the lower strain rate limit of the plateau at the remarkably small value of $10^{-2} \mathrm{~s}^{-1}$. We attribute the very low strain rate at the onset of the plateau to slow dissociation/recombination dynamics for these Pluronic micellar systems.

2. The thixotropy as seen in the time dependent stress at fixed shear rate following startup

3. The inflected temperature dependence of the low shear rate viscosity, an effect which correlates with the temperature dependence of the micellar nematic order,

4. The dual order parameters of the nematic state, as sensed by the deuterated phenylalcohol probe molecule. We attribute this duplicity to partitioning between the micellar corona and core.

5. The bewildering array of spatially-dependent shear rates and associated fluctuations. Fluctuations under shear banding conditions are not by themselves remarkable effects and are now accepted as a fairly universal property of shear banding dynamics in wormlike micelle systems, though the variety we find exceeds expectations.

6. The lack of a simple correspondence between shear banding and the spatially dependent order parameter. This discrepancy between nematic or- 
der and fluid properties (which had been pointed out for a previous different system, and confirmed here), only serves to emphasise that our models for shear banding and band dependence on molecular organisation, are far from understood. 


\section{Bibliography}

[1] P Fischer. Time dependent flow in equimolar micellar solutions: transient behaviour of the shear stress and first normal stress difference in sheer induced structures coupled with flow instabilities. Rheol. Acta, 39(3):234-240, AUG 2000. EuroRheo 99-1 Meeting, SOPHIA ANTIPOLIS, FRANCE, MAY 03-07, 1999.

[2] H Rehage and H Hoffmann. Viscoelastic surfactant solutions - model systems for rheological research. Mol. Phys., 74(5):933-973, DEC 101991.

[3] A Khatory, F Lequeux, F Kern, and SJ Candau. Linear and nonlinear viscoelasticity of semidilute solutions of wormlike micelles at high-salt content. Langmuir, 9(6):1456-1464, JUN 1993.

[4] TM Clausen, PK Vinson, JR Minter, HT Davis, Y Talmon, and WG Miller. Viscoelastic micellar solutions - microscopy and rheology. Journal of Physical Chemistry, 96(1):474-484, JAN 91992.

[5] K Holmberg, B Jonsson, and B Kronberg. Surfactants and polymers in aqueous solution. Wiley, 2003.

[6] DF Evans and H Wennerstrom. The Colloidal Domain. Wiley-VCH, 1999.

[7] F Lequeux and SJ Candau. Structural properties of wormlike micelles. Theoretical Challenges in Complex Fluids, 339(8), AUG 1996.

[8] E Cappelaere, JF Berret, JP Decruppe, R Cressely, and P Lindner. Rheology, birefringence, and small-angle neutron scattering in a charged micellar system: Evidence of a shear-induced phase transition. Physical Review E, 56(2):1869-1878, AUG 1997. 
[9] H Rehage and H Hoffmann. Rheological properties of viscoelastic surfactant systems. Journal of Physical Chemistry, 92(16):4712-4719, AUG 111988.

[10] J Appell, G Porte, A Khatory, F Kern, and SJ Candau. Static and dynamic properties of a network of wormlike surfactant micelles (cetylpyridinium chlorate in sodium-chlorate brine). Journal De Physique II, 2(5):10451052, MAY 1992.

[11] RW Mair and PT Callaghan. Observation of shear banding in worm-like micelles by NMR velocity imaging. Europhys. Lett., 36(9):719-724, DEC 201996.

[12] RW Mair and PT Callaghan. Shear flow of wormlike micelles in pipe and cylindrical couette geometries as studied by nuclear magnetic resonance microscopy. Journal of Rheology, 41(4):901-924, JUL-AUG 1997.

[13] MM Britton and PT Callaghan. Nuclear magnetic resonance visualization of anomalous flow in cone-and-plate rheometry. Journal of Rheology, 41(6):1365-1386, NOV-DEC 1997.

[14] AF Mendez-Sanchez, MR Lopez-Gonzalez, VH Rolon-Garrido, J PerezGonzalez, and L de Vargas. Instabilities of micellar systems under homogeneous and non-homogeneous flow condition. Rheol. Acta, 42(1-2):56-63, JAN 2003.

[15] JB Salmon, L Becu, S Manneville, and A Colin. Towards local rheology of emulsions under couette flow using dynamic light scattering. European Physical Journal E, 10(3):209-221, MAR 2003.

[16] JB Salmon, A Colin, S Manneville, and F Molino. Velocity profiles in shear-banding wormlike micelles. Phys. Rev. Lett., 90(22):228303, JUN 6 2003.

[17] JB Salmon, S Manneville, and A Colin. Shear banding in a lyotropic lamellar phase. I. time-averaged velocity profiles. Physical Review E, 68(5, Part 1):051503, NOV 2003. 
[18] JB Salmon, S Manneville, and A Colin. Shear banding in a lyotropic lamellar phase. ii. temporal fluctuations. Physical Review E, 68(5, Part 1):051504, NOV 2003.

[19] S Manneville, L Becu, and A Colin. High-frequency ultrasonic speckle velocimetry in sheared complex fluids. European Physical Journal-Applied Physics, 28(3):361-373, DEC 2004.

[20] S Manneville, JB Salmon, and A Colin. A spatio-temporal study of rheooscillations in a sheared lamellar phase using ultrasound. European Physical Journal E, 13(2):197-212, JAN 2004.

[21] L Becu, S Manneville, and A Colin. Spatiotemporal dynamics of wormlike micelles under shear. Phys. Rev. Lett., 93(1):018301, JUL 2004.

[22] JP Decruppe, R Cressely, R Makhloufi, and E Cappelaere. Flow birefringence experiments showing a shear-banding structure in a CTAB solution. Colloid Polym. Sci., 273(4):346-351, APR 1995.

[23] R Makhloufi, JP Decruppe, A Aitali, and R Cressely. Rheooptical study of worm-like micelles undergoing a shear banding flow. Europhys. Lett., 32(3):253-258, OCT 201995.

[24] E Fischer and PT Callaghan. Shear banding and the isotropic-to-nematic transition in wormlike micelles. Physical Review E, 64(1, Part 1):011501, JUL 2001.

[25] V Schmitt, F Lequeux, A Pousse, and D Roux. Flow behavior and shearinduced transition near an isotropic-nematic transition in equilibrium polymers. Langmuir, 10(3):955-961, MAR 1994.

[26] ME Cates. Reptation of living polymers - dynamics of entangled polymers in the presence of reversible chain-scission reactions. Macromolecules, 20(9):2289-2296, SEP 1987.

[27] EK Wheeler, P Fischer, and GG Fuller. Time-periodic flow induced structures and instabilities in a viscoelastic surfactant solution. J. NonNewtonian Fluid Mech., 75(2-3):193-208, MAR 1998. 
[28] YT Hu, SQ Wang, and AM Jamieson. Rheological and flow birefringence studies of a shear-thickening complex fluid - a surfactant model system. Journal of Rheology, 37(3):531-546, MAY-JUN 1993.

[29] YT Hu and EF Matthys. Characterization of micellar structure dynamics for a drag-reducing surfactant solution under shear - normal stress studies and flow geometry-effects. Rheol. Acta, 34(5):450-460, SEP-OCT 1995.

[30] R Bandyopadhyay, G Basappa, and AK Sood. Observation of chaotic dynamics in dilute sheared aqueous solutions of CTAT. Phys. Rev. Lett., 84(9):2022-2025, FEB 282000.

[31] WM Holmes, MR Lopez-Gonzalez, and PT Callaghan. Fluctuations in shear-banded flow seen by NMR velocimetry. Europhys. Lett., 64(2):274280, OCT 2003.

[32] MR Lopez-Gonzalez, WM Holmes, PT Callaghan, and PJ Photinos. Shear banding fluctuations and nematic order in wormlike micelles. Phys. Rev. Lett., 93(26):268302, DEC 312004.

[33] MM Britton and PT Callaghan. Shear banding instability in wormlike micellar solutions. European Physical Journal B, 7(2):237-249, JAN 1999.

[34] SM Fielding and PD Olmsted. Spatiotemporal oscillations and rheochaos in a simple model of shear banding. Phys. Rev. Lett., 92(8):084502, FEB 272004.

[35] SM Fielding and PD Olmsted. Flow phase diagrams for concentrationcoupled shear banding. European Physical Journal E, 11(1):65-83, MAY 2003.

[36] L Guo, RH Colby, and P Thiyagarajan. Temperature and hydrophobic alcohol-induced structural changes of pluronics micelles. Physica BCondensed Matter, 385(Part 1 Sp. Iss. SI):685-687, NOV 15 2006. 8th International Conference (ICNS 2005), Sydney, AUSTRALIA, NOV 27-DEC $02,2005$. 


\section{Chapter 6}

\section{Extensional flow investigations with magnetic resonance}

\subsection{Introduction}

In complement to the capabilities of Rheo-NMR in the study of shear effects in complex fluids, this chapter considers the potential for NMR to be used to study extensional flow. In particular this shall be from the perspective of a particular geometry, the semi-hyperbolic converging channel, though the fourroll mill has been used previously to investigate liquid-crystalline polymers in this laboratory. Extensional flow studies provide insight into material rheology from a perspective that is rarely investigated routinely in great depth due to the difficulty inherent in experimental techniques designed to do the job, but also due to a fundamental confusion and lack of consistency between experimentalists in the field $[1,2]$. Newtonian fluids demonstrate a simple relationship between the viscosity in both the shear and extensional geometries, namely the Trouton relationship. For an extensional viscosity, $\eta_{E}$, of a material undergoing uniaxial deformation, defined by the quotient of the first normal stress difference and the extension rate, $\dot{\varepsilon}$, the Trouton relationship states that this value will be simply three times the shear viscosity [3] conventionally measured

$$
\eta_{E}=\frac{\sigma_{z z}-\sigma_{y y}}{\dot{\varepsilon}}=3 \eta
$$

Departure from this well-established result in which the Trouton ratio is 3 
$\left(\eta_{E} / \eta=3\right)$ occurs in instances where the extension and compressional deformation is biaxial (though the Trouton ratio is well known in that situation) and in viscoelastic, non-Newtonian fluids (although very small rates of strain lead to the limiting case as presented for Newtonian fluids). For the simple reason that extensional flow parameters are usually only anomalous or novel for non-Newtonian fluids, the strain history, in the form of a full constitutive equation for the material is necessary. This is rarely exactly provided in real situations [4-6]. The lack of controllability of flows in apparatuses designed to generate extensional flow is a crucial limiting factor, as none of the more common devices used are based on a predetermined flow field [7-13]. In the case of the four-roll mill a stagnation point is the centre of region of extension, though under the assumption the fluid is non-Newtonian, the flow field and hence strain history for the sample is poorly known. Other dynamic problems detract from other devices such as the opposed jet and filament stretching rheometers [14].

\subsection{The semi-hyperbolic converging die}

Compromise is everywhere in the study of extensional flow. Controllability and stability are often played off against repeatability, though the more repeatable or recyclable a geometry is in imparting deformation, the less analagous to practical situations the process becomes. Flow geometries which minimise some of the undesirable dynamical effects and allow increased knowledge of the strain history are the axisymmetric contraction (with sharp step down in the radius of the pipe [15]) and the semi-hyperbolically converging die [16]. In pumping a fluid of interest through a specially designed contraction, the compression required in the tranverse directions to flow are applied to achieve expansion in the other. This process is highly repeatable (given enough time is allowed for sample to relax fully) and the semi-hyperbolic converging die, in particular, minimises turbulent effects in regions the sample traverses prior to entering a critical experimental zone. Ambiguity and indecision in the literature indicate that no authoritative method of extracting an extensional viscosity, $\eta_{E}[8,17]$, has been settled on universally. Magnetic resonance investigations here, though, are no different to shearing a sample in a Rheo-NMR cell: experiments are macroscopically strain-rate controlled, rather than stress being the independent 
variable, and as such we do not extract an extensional viscosity. Despite this, magnetic resonance can, as always, be of importance in measuring velocity profiles and molecular alignment.

One other advantage of a flow cell, compared to other geometries, including those which "grab" or physically stretch a sample, is reversibility. By the tenets of fluid mechanics of non-turbulent flows of Newtonian fluids, flow should be perfectly reversible through a pipe. Samples which we are likely to take interest in, however, will be non-Newtonian, though it is conceivable that important information, or at least another type of experiment, may be on offer in running fluid through an "expanding" rather than contracting flow.

\subsubsection{Design of a converging die}

The design of a semi-hyperbolically converging die (SHCD) shall here be described, with fluid mechanical principles outlined first, followed by the adaptation of a sufficient geometry to the specifications required by a magnetic resonance experiment. The Venturi effect describes the properties of materials flowing through a constriction in a pipe, observing that the fluid pressure decreases with the radius of the pipe [18], and the velocity of the fluid increases in order to satisfy the continuity equation.

Most critical in the design is the extension rate, $\dot{\varepsilon}$, able to be generated by a converging flow. Temporarily ignoring the no-slip boundary condition for fluid velocity at the walls of the pipe, we would like to impose an extension rate which is constant along the length, $z$, of the SHCD, in particular on its central axis [19].

$$
\dot{\varepsilon}=\frac{\partial v_{z}}{\partial z}
$$

which, if able to be satisfied, results in a velocity profile of

$$
v_{z}=\dot{\varepsilon} z+v_{i}
$$

given that the inlet velocity is $v_{i}$. This inlet velocity will therefore determine the extension rate attained for a given experiment given an apparatus of fixed inlet and outlet diameters. The volumetric flow rate, $Q$, which is constant at 
any plane transverse to the axis regardless of pipe radius, $R$, may be written as an integral of axial velocities over that plane.

$$
Q=2 \pi \int_{0}^{R} d r \cdot v_{z} r=\pi v_{z} R^{2}
$$

We are therefore able to reveal a functional form of the SHCD in order to produce the desired extension rate

$$
R^{2}(z)=\frac{Q}{\pi\left(\dot{\varepsilon} z+v_{i}\right)}=\frac{C}{z+a}
$$

where

$$
a=\frac{v_{i}}{\dot{\varepsilon}} ; \quad C=\frac{Q}{\pi \dot{\varepsilon}}
$$

For completeness, prior to determining the magnitude of parameters for a particular realisation of the geometry, it is worth noting how much time will be spent in the critical region of the pipe corresponding to constant extension. This may be calculated by first determining the position of a fluid element as a function of time

$$
\begin{aligned}
v_{z}=\frac{d z}{d t} & =\dot{\varepsilon} z+v_{i} \\
\frac{d z}{z+a} & =\dot{\varepsilon} d t \\
\log k(z+a) & =\dot{\varepsilon} t \\
z & =k^{-1} \exp \dot{\varepsilon} t-a \\
& =a[\exp \dot{\varepsilon} t-1]
\end{aligned}
$$

where, in the final step, we have set the arbitrary constant of integration $k=$ $a^{-1}$, so as to have the effect of setting the inlet position $z=0$. By now inverting this expression we can state that the time at which the time a fluid element enters the constriction is $t=0$, and leaves at time

$$
t_{\text {out }}=\frac{1}{\dot{\varepsilon}} \log \left(\frac{z_{\text {out }}}{a}+1\right)
$$




\subsubsection{Realisation of a converging die for magnetic reso- nance}

Physical constraints shall dominate the determination of the functional form of the SHCD for real experiments. After the magnetic field gradient coils typically used are placed in the bore of a $400 \mathrm{MHz}$ magnet, the remaining space available for a probe and r.f. coil (see Chapter 3) is $39 \mathrm{~mm}$, though this is further reduced by the r.f. coil itself. The coil chosen has an inner diameter of $20 \mathrm{~mm}$, and this is therefore the maximum horizontal dimension of the SHCD, including pipe wall materials. In other research on flow through contractions, an inlet-tooutlet diameter of 3:1 has been prevalent, and for reasons that will be explained in more detail in discussing construction and testing in section 6.2.2, the inner diameter at the SHCD inlet was chosen to be $9 \mathrm{~mm}$, the corresponding diameter at the outlet was chosen to be $3 \mathrm{~mm}$, over an axial distance of $10 \mathrm{~mm}$. These two co-ordinates (see fig. 6.1) lying on the curve, $R^{2}(z)=C /(z+a)$, fully determine the two parameters $C=405 / 16$ and $a=5 / 4 \mathrm{~mm}$.

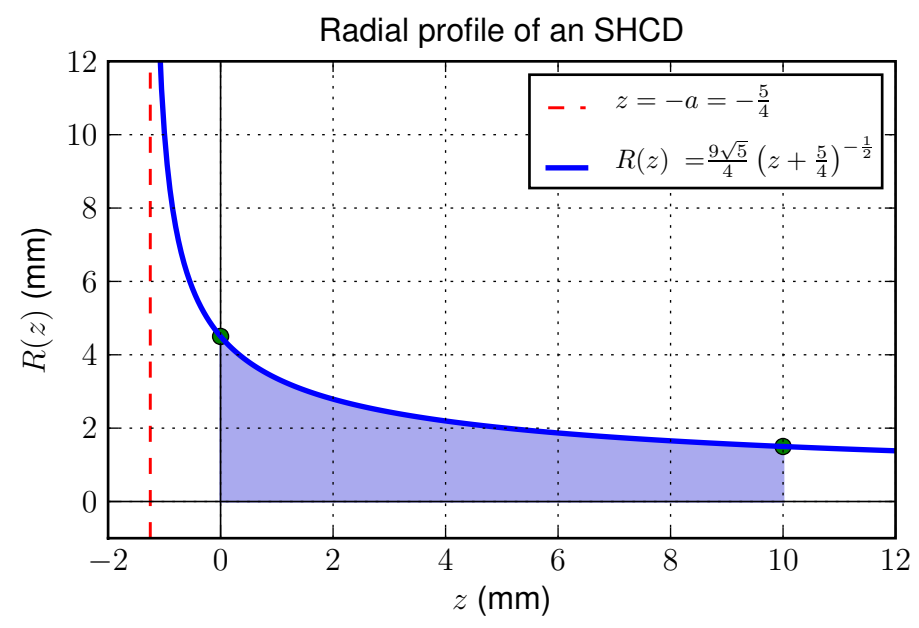

Figure 6.1: A functional form for our semi-hyperbolically converging die. The two points specified as being suitable dimensions for a magnetic resonance experimental apparatus determine appropriate coefficients and offsets. The shaded region shown is the shape needed for our die volume. 


\section{Materials and construction}

In the first instance, a converging pipe was designed and built from a potting resin set around a negative of the prescribed shape, which was in turn inlaid in a $20 \mathrm{~mm}$ cylindrical acrylic block for centering and attaching of pipes leading into and out of the device. A closed-cycle pump was employed to drive the fluid around a loop passing through the magnet, though in subsequent incarnations, this arrangement was replaced by a single-pass (though repeatable) pumping system, for the main reason of sample conservation. A pumping system in closed-cycle mode will find pumping the sample through wider pipes easier, though this has the consequence of requiring more sample to fill the loop in the first place. The resin SHCD was particularly prone to being a collection point for bubbles that were particularly difficult to dislodge, and as such the device was only used for preliminary proof-of-concept for degassed water, having been doped to reduce surface tension.

Specially-made drill bits were constructed in order to generate a secondgeneration SHCD device. By drilling and milling a hole "inside-out", so to speak, a converging pipe was gouged from a PEEK (polyetheretherketone) at the prescribed radius, point-by-point along the axis of the pipe. As the importance of laminar flow prior to entry had been noted from the previous construction attempt, a long $(L / D \sim 30)$ teflon tube was affixed before the inlet. While this design was temporarily successful, the problem of ensuring the flow conditions and strain history of the sample were ideal remained a problem. Joins at the interface of the lead-in tube and the SHCD had a non-negligble effect, in addition to potential turbulence caused by a sharp change in pipe radius at the join. Though no roughness could be felt by hand on the surface, in all fabrication attempts, at least one imperfection in the channel surface was visible through magnetic resonance imaging of the channel space, having been filled with water. It was therefore perceived that an alternative to the milling process was preferable in the long-term as visible striations were present inside the SHCD. The primary suspected cause of the deformities in the inner surface was non-homogeneous (or even outright) swelling and contraction of the pipe material due to heat in the machining process, and the resultant cooling phase. Another method of inside-out drilling was also investigated: blades were cut out 
of a stainless steel negative, which resulted in the shape being drilled out of a block of material in one pass.

\section{Final design}

Glass is a suitable material for use in both magnetic-resonance- and rheologyoriented experiments, due to its transparency, lack of electrical conductivity, and lack of reactivity. The only relevant properties of glass which are unfavourable for use in a device are its brittleness (easily negated with a little care) and the difficulty it presents in crafting a precisely defined shape. Following attempts at fabricating an SHCD in multiple parts, with multiple materials, a design was devised which called for a monolithic glass piece. By lathing the correct functional form for the radius of the end of a graphite rod (which it should be noted may also suffer from non-homogenous thermal expansion), glass could then be drawn down onto its surface. In addition to the negative only being designed to provide the correct shape for the contraction segment of the pipe, a small region leading up to the technical inlet was allowed for. This section was rounded so as to reduce any effects due to any sudden change in pipe radius. After shaping the glass constriction was fused with a long straight glass pipe $(L / D \sim 15)$; the total profile of this object is shown in fig. 6.2, the form was confirmed by imaging, a portion of which is seen in fig. 6.3.

With regards to the pumping system, a more efficient system in terms of sample volume required was needed, in particular a move away from a closedloop flow system being preferable. The standard drive-shaft used in Rheo-NMR experiments integrates into the design in a way that uses the Rheo-NMR system motor to rotate the shaft, which couples to a screw-thread piston shunting sample from a reservoir through the SHCD (see fig. 6.4). This arrangement has the benefits of requiring a lower redundant-volume to sample-volume ratio, meaning that the sample is not required to pass through any length of very thin tubing, ensuring a strain history which is as gentle as can be practical. Very simply, it also allows a reversal of the flow system with a reversal in direction of the Rheo-NMR motor, which can be implemented in software rather than requiring manual hardware switching. This ability makes the experimental procedure considerably less error-prone, as well as requiring far less supervision 
than would otherwise be necessary. The number of hours of time and effort saved are considerable.

To connect the glass tubing comprising the monolithic piece to the reservoir, an acetal sheath houses the tube (which protrudes 1-2 $\mathrm{mm}$ from the top), with a screw-down top capping it. Inset into the screw-top is an annulus of silicone rubber, which when hard pressed against the glass tube end provides a seal and connection with the outlet from the reservoir (or closed-cycle system tubing if necessary). This housing for the glass pipe and constriction serves several purposes (fig. 6.5), including providing stability, access, location and connection. A small o-ring cushion between the SHCD and the step of the sheath on which it rests provides adequate resistance when the screw top pushes the glass pipe downwards, and protects the SHCD from any hard corners.

\subsection{Basic flow issues through the glass SHCD}

\subsubsection{Limitations}

\section{Velocimetry}

In the same vein as the variety of experiments involved in Rheo-NMR help fill in the blanks for experiments involving shear deformation, we aim to get a grounding in the use of such techniques in extensional flow geometries. With the intrinsic increase in velocities as fluid traverses the pipe constriction in an SHCD, the considerations are typical: in order to resolve differences in low velocities near the tube inlet, the maximum available gradient is important; once chosen however, the range of observable displacements (field-of-flow) is determined by the division of the applied gradient into " $q$ "-steps, though this number of steps affects experimental time strongly.

\section{Spectroscopy}

In the spectroscopic category of experiments it is more important to ask the required resolution to observe a spectral effect, for example the quadrupolar splitting expected for an aligned fluid containing deuterium nuclei. However small the required resolution, the acquisition time and hence the time spent 


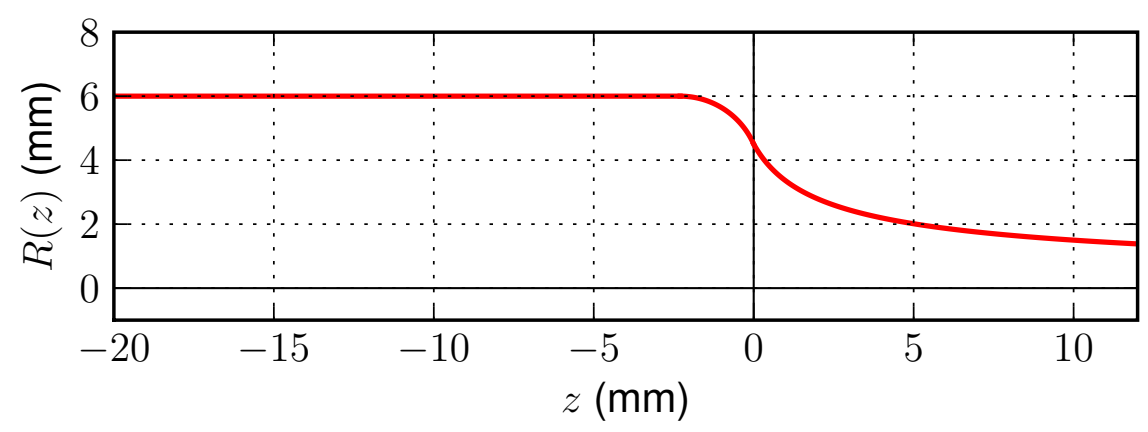

Figure 6.2: Our final design for a converging die. A long $(L / D \gtrsim 20)$ straight section of glass pipe is joined to the semi-hyperbolic constriction with a curve comprising a circular arc.

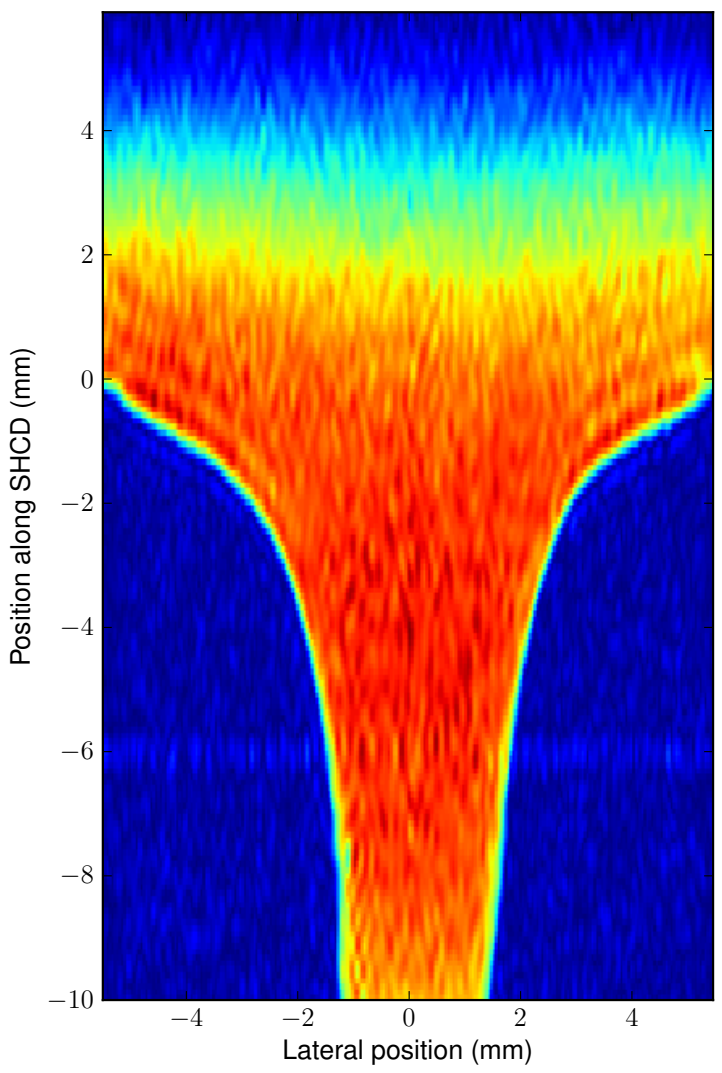

Figure 6.3: The shape of the converging die as seen by a simple magnetic resonance density image 


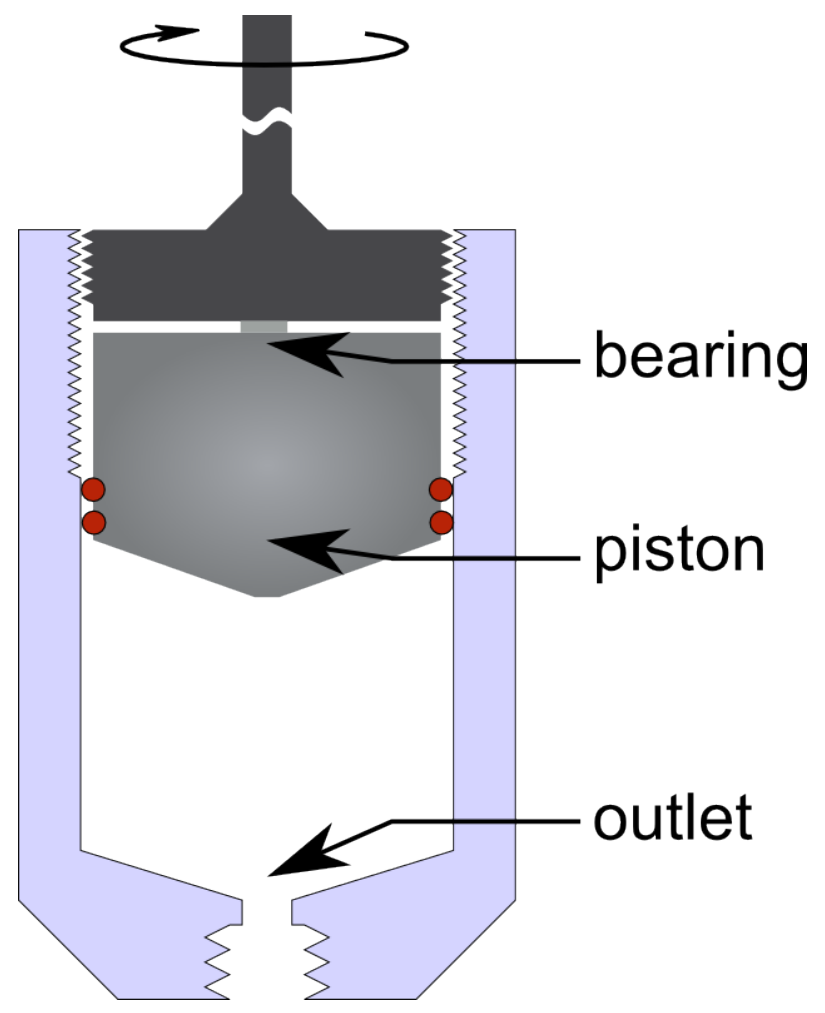

Figure 6.4: The reservoir and piston of the extensional flow cell (not to scale). The standard Rheo-NMR motor atop the magnet turns the screw shown; a bearing between the rotating drive and piston allows the piston to simply move back and forth; o-rings provide a seal between the piston and reservoir wall. Forward and reverse modes are provided by the motor, allowing software resetting and control.

by a nuclear species in a characteristic volume sensitive to the experiment (cf. eqn(6.11)), must exceed its inverse.

\section{Rheology}

From a purely mechanical standpoint, however, the minimal inlet velocity available will be restricted by the extension rate range desired, and whether it provides an observable effect. The maximal inlet velocity will be restricted by the velocity at which turbulence may creep in to the flow, whether it is due to fast flow incident on the slightly curved constriction point of the pipe, or the more sharp-edged outlet at the narrow end of the SHCD. An upper limit may present itself indirectly owing to the reduction in experimental time available due to 


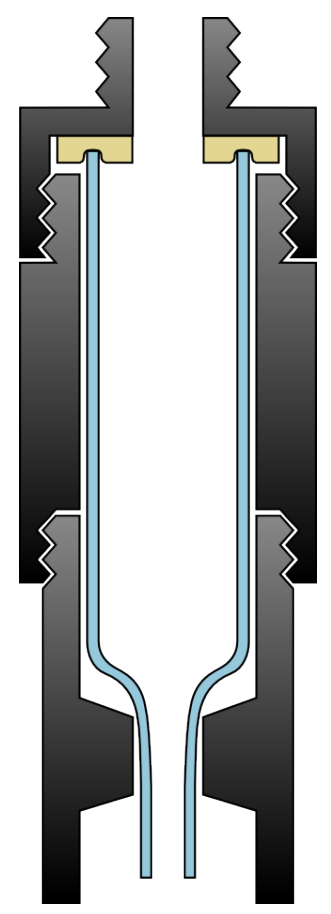

Figure 6.5: A glass SHCD, with a long uniform pipe inlet (not to scale). The modular acetal sheath gives protection and access to the glasswork, as well as connection to the reservoir above, and centring the device in the rf coil.

the finite extent of the reservoir - faster flow and higher throughput reduce this available time drastically. Signal averaging of repeated experiments may help in some cases, though if the observable is itself a result of some transient effect, re-establishing the flow would be quite useless. In general, however, we hope to be performing experiments at modest Reynolds numbers which help avoid turbulence issues and prolong experimental time available, which in turn helps guarantee we have established a steady flow profile where possible.

\section{Apparatus, materials and samples}

Besides flow-rate limitations, technical issues that are worth keeping in mind are the nature of materials used, and the way in which they connect and directly interact. The o-rings around the piston providing a seal between piston and reservoir may be lubricated, where it can be expected that the very small amount of lubricant applied will not slowly creep into the sample as the piston goes upand-down the reservoir wall repeatedly. A small screw-hole in the wall of the 
reservoir near its bottom also gradually degrades the o-ring through friction, which means the o-ring must be replaced periodically to protect against leaks up the side of the piston wall. Two main pipe connections are present in the system described, being those between the reservoir and the sheath, and the SHCD outlet and the drainage tubing. Teflon taping is generally applied to the screw thread in the first instance, and depending on the drainage tube materials, various ways are used to secure the second. The silicone rubber washer providing a seal at the top of the glass pipe within the sheath could be considered a third main connection, the seal is regularly replaced, in particular at the change of sample to avoid contamination.

Sample volume requirements are most vital to bear in mind in experiment design with this device. Steady-state flow (if possible) is the ideal experimental regime, and to this end priming the entire assembly, at least to the bottom of the SHCD, is desirable. Once filled, only the volume above the exit-point of the reservoir is available as throughput for the experiment. The piston may act upon this sample material for however long the screw-thread takes to drive this head into the sheathed inlet glass pipe. As such the minimum sample needed, in addition to that of the actual experimental volume is

$$
V_{\text {min }}=\pi(6 \mathrm{~mm})^{2} \cdot(160 \mathrm{~mm})=18 \times 10^{3} \mathrm{~mm}^{3}=18 \mathrm{~mL}
$$

Our SHCD design, incorporating an $\sim 80$ turns screw-thread piston of $0.5 \mathrm{~mm}$ pitch, and having a maximum screw speed of $14 \mathrm{~Hz}$, would exhaust its reservoir in around $5 \mathrm{~s}$ at maximum rate. Knowing that, in general, at least one order of magnitude more in experimental time will be required at a minimum, we restrict our preliminary investigations to a maximum rotation rate for the drive of $1 \mathrm{~Hz}$, and hence (not wanting to reach the screw limits at any time) a minimum experimental time of $\lesssim 80 \mathrm{~s}$.

\section{Temperature control}

The realm of temperature-controlled experiments is largely unavailable with this apparatus, ambient conditions being the only control used. Besides there being very little air-flow available around the shaft/sample reservoir region (see fig. 6.6) The reason for this is that the fluid is passing through the apparatus 
having originated in the ambient conditions of the reservoir at the upper region of the shim set of the NMR magnet system. With the wide range of locations through which sample material passes on its course from the piston source to a flask sink, maintenance of a uniform sample temperature in the case of artificial heating or cooling would be extremely difficult. Even under ambient conditions there is no guarantee of any extraordinary temperature homogeneity. While this is less than ideal, in particular where comparisons are made between extensional flows and those shear flows in the cylindrical Couette of chapter 5, as a proofof-concept the geometry is acceptable and many interesting phenomena are still to be found.

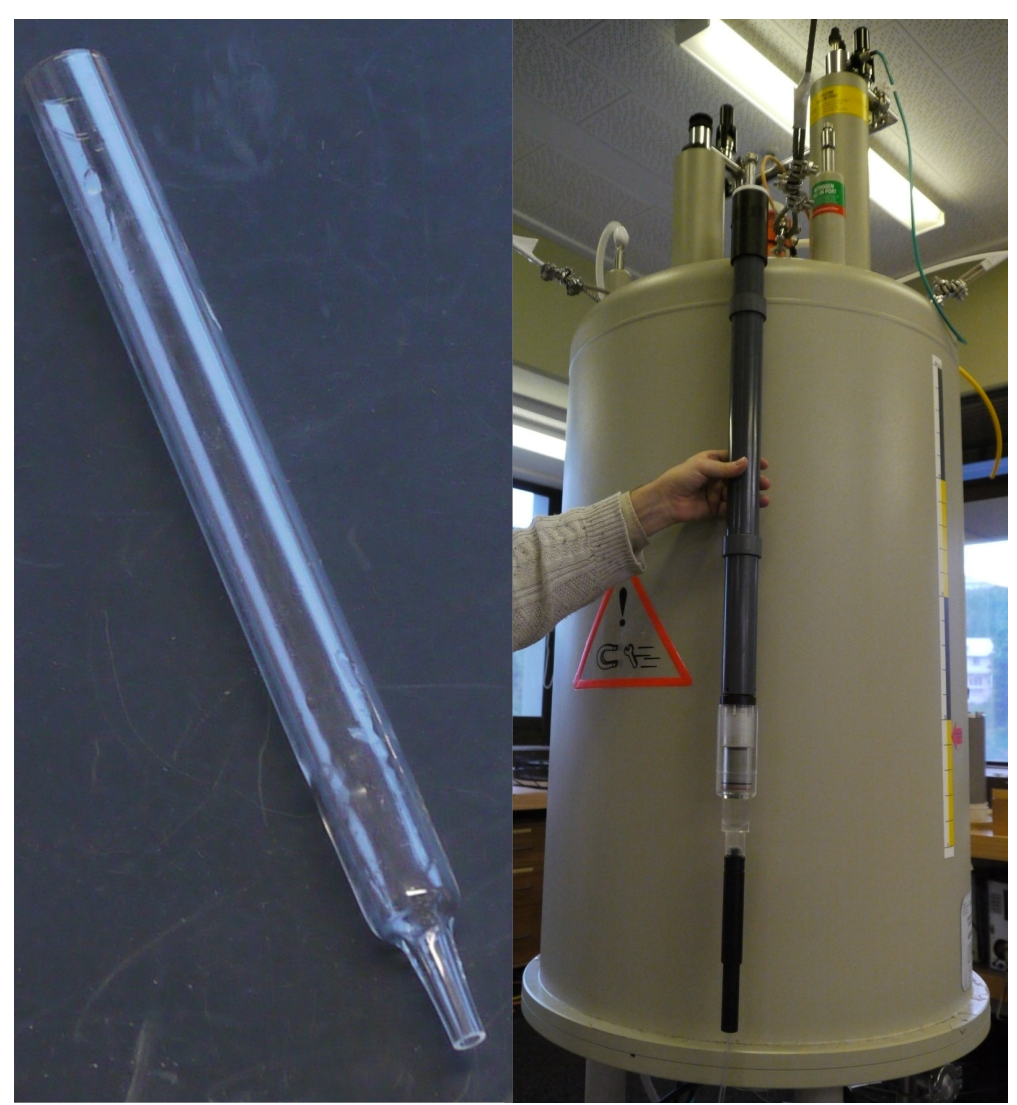

Figure 6.6: (left) The glass SHCD device, which locates itself in the black acetal housing shown at the bottom of (right) the custom-built Rheo-NMR drive shaft with screw-thread piston, reservoir, SHCD housing, and outlet.

Having noted these difficulties, the temperature can be roughly estimated as being around $(21 \pm 2)^{\circ} \mathrm{C}$, since this corresponds to the air-conditioner controlled 
room-temperature and the setting of the gradient coil cooling system.

\subsection{SHCD contraction flows}

As water is Newtonian, freely available and non-corrosive to any parts of our apparatus, it is our test sample of choice. Following various numerical calculations performed by other groups [19, 20], the flow profiles we expect through the SHCD will be similar, though perhaps not identical to, the schematic shown in figure 6.7. In particular, it would be expected that the rounded wall at our inlet would have an effect, and preliminary experiments will be required to determine just to what degree this is the case. Such experiments will also give some indication as to the ease or difficulty the Rheo-NMR system and screw-thread piston will have in pumping fluids, while bearing in mind that generally, samples of interest will be orders of magnitude more viscous than water.

\subsubsection{Expectations based on prior investigations}

As the basis for the realisation of a shear-free, and hence purely extensional flow, we require that the fluid velocity gradient at points lying on the central axis of an SHCD should be zero with respect to all radial directions, and take the value $\dot{\varepsilon}$ with respect to the axial direction. The extent of the region that this condition holds may not necessarily be cylindrical, though we might expect it to be at least circular at any cross-section of the SHCD. Firstly, we try to establish that our converging die does indeed generate a linearly increasing velocity profile along the axis, and then establish the degree to which the new geometry extends the shear-free region, compared to the infinitesimally thin axial line at which shear disappears in a uniform pipe flow.

Figure 6.7 shows schematically typical profiles (quantitave variations occur with both position in the SHCD and with input flow rate) as measured by Shirakashi et al. [21] and as simulated by Feigl et al. [19, 22]. Their respective works show quite convincingly that the flow profile over cross-sections within the SHCD range flatten considerably near the pipe axis, and the exclusion of shear is increased with increasing flow rate (which in turn is proportional to extension rate). The data of Shirakashi suggests that a nearly totally shear-free 

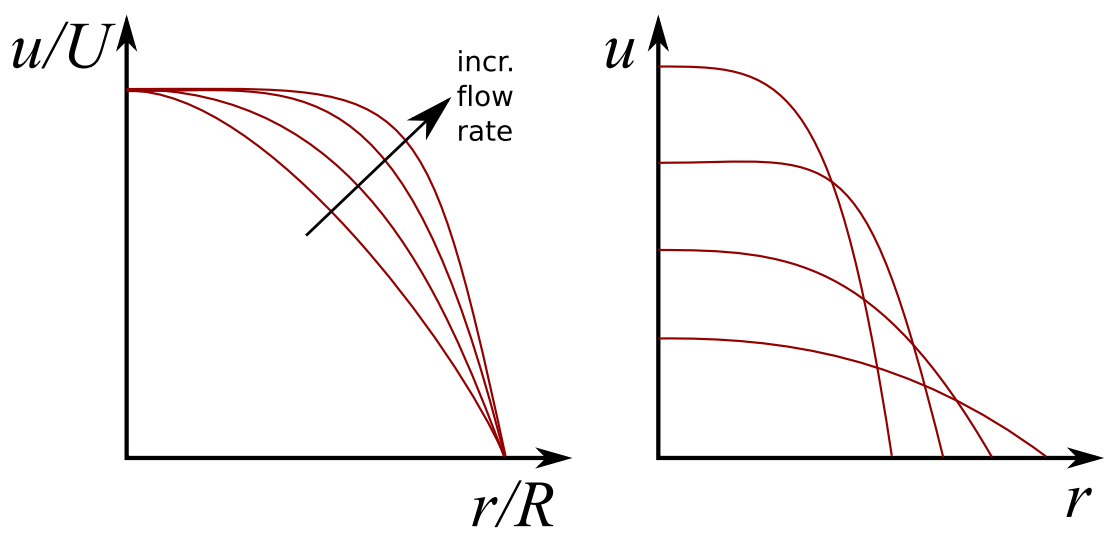

Figure 6.7: [Schematics reproduced from Shirakashi (left), and Feigl (right)] Two flow profiles for fluids in a converging channel. There are minor differences in dimensions for the geometries. The work of Shirakashi et al. (left) is obtained on water flow by laser Doppler velocimetry, and shows the flow profile changing with flow rate at a fixed position. The profiles of Feigl et al. (right) shows numerical simulation data of an LDPE melt at fixed flow rate at several points along the SHCD.

region may be generated out to a normalised radius of between 0.4 (at lower extension rates) and 0.6 (at higher extension rates), values which seem at least amenable to our investigations. Their presented data are fit with a 'power law' relationship, with power law exponent, $s$, of

$$
\frac{u}{U}=1-\left(\frac{r}{R}\right)^{s}
$$

Expected axial velocities along the centre-line of the ideal SHCD (showing a linear increase in velocity between the inlet and outlet) are shown schematically in figure 6.8 .

\subsubsection{Experimental velocimetry of flow through an SHCD}

The degree to which our contraction approaches these ideals seen above remains to be seen, however. Bruker Paravision software, and a spin-echo velocimetry method was used to acquire velocity maps over a $1 \mathrm{~mm}$ thick slice in a plane containing the flow axis and one transverse direction (a "vertical slice"). Using a range of screw-thread driven piston rates between $0.08 \mathrm{rev} / \mathrm{s}$ and $0.56 \mathrm{rev} / \mathrm{s}$ (linear piston speed range of $0.04 \mathrm{~mm} / \mathrm{s}$ and $0.25 \mathrm{~mm} / \mathrm{s}$ ), velocity maps over the 


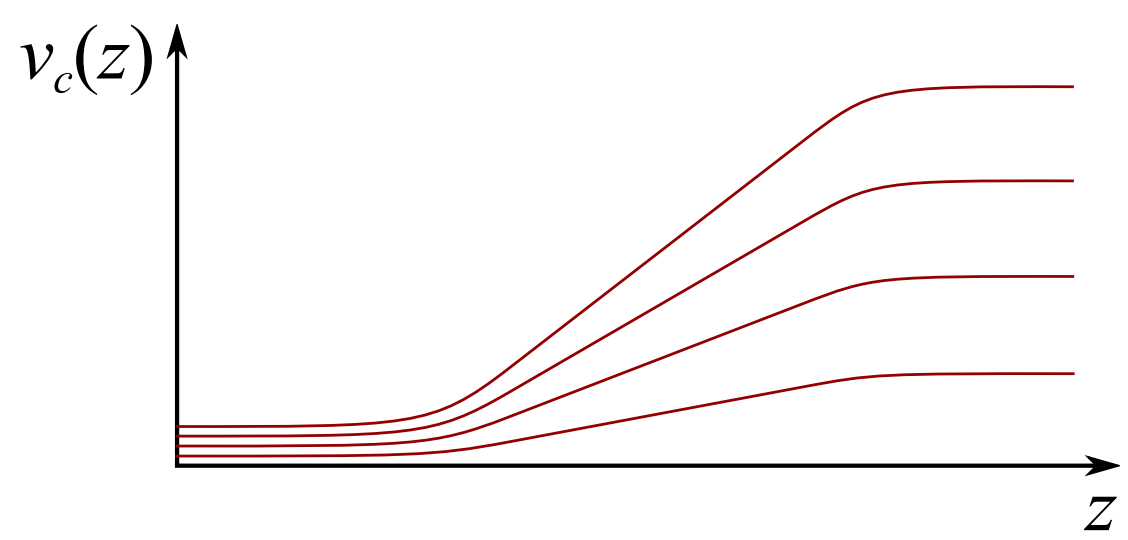

Figure 6.8: Between the inlet and outlet, the ideal behavior for fluid following along the stream line lying on the central axis is to experience uniform acceleration (with position). Outside of this region, fluid is expected to maintain constant velocity in the straight pipes leading in and out of the critical region.

vertical slice were obtained for a water sample (fig. 6.9) doped with gadolinium chloride so as to minimise experimental repetition times. In fig. 6.11, the linear progression of velocity with space is seen for seven equally spaced piston speeds in the stated range, scaled in a way so as to show the velocity of the fluid as a fraction of the field-of-flow provided by the experimental parameters (half the field-of-flow being positive, half being negative). Immediately, it can be seen that the velocity profile along the axial line is approximately linear in that coordinate, precisely the constraint for extensional flow: $v_{z} \propto z$. The coincidence of each velocity profile on such a scaled diagram is also reassuring. Finally, flow profiles across the pipe are shown in fig. 6.13.

The vertical slice velocity maps were repeated when Pluronic P105 solution was pumped through the SHCD (fig. 6.10), the central velocity profiles for P105 are shown in fig. 6.12, and the cross-pipe flow profiles are shown in fig. 6.14. 

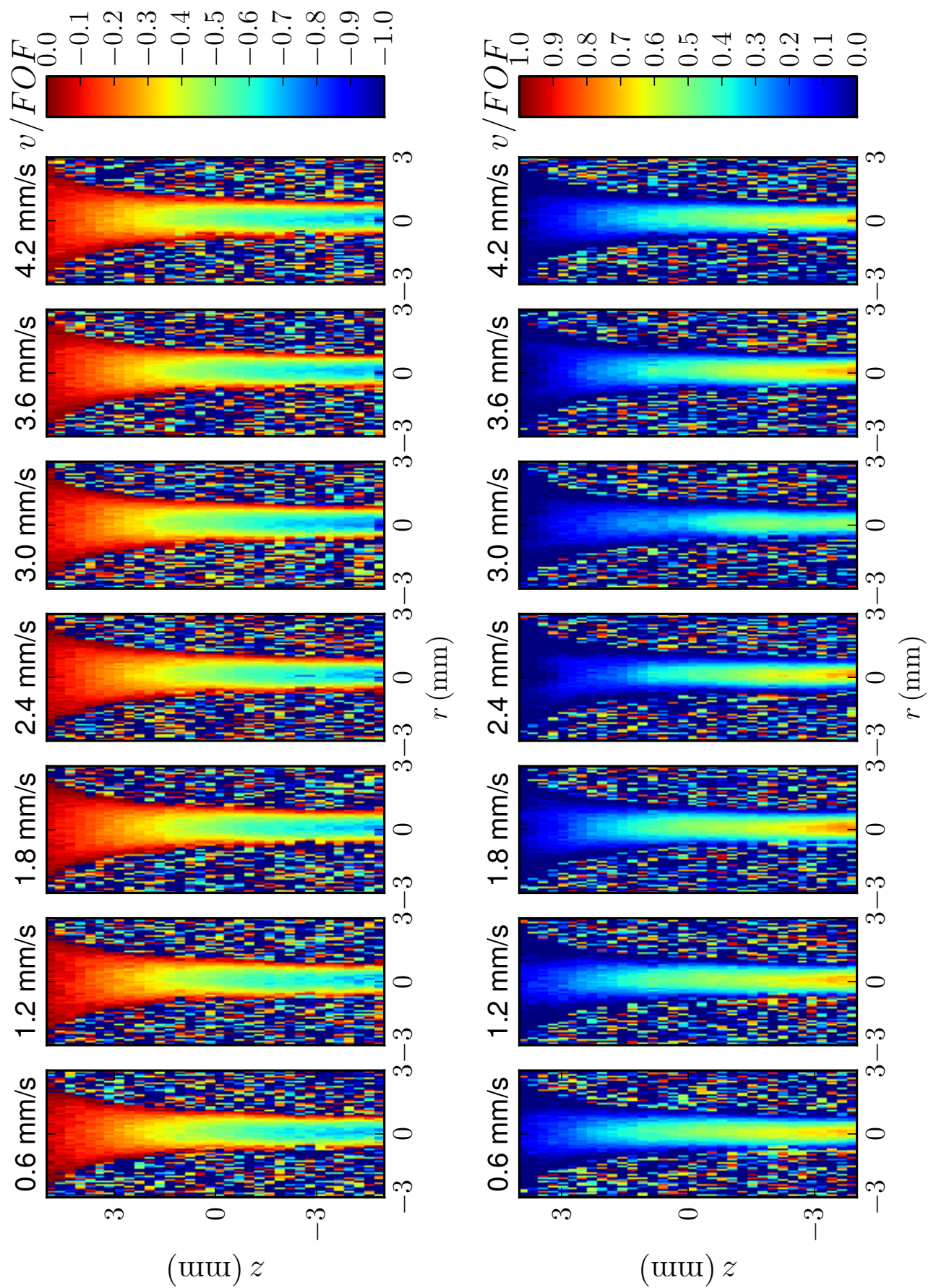

Figure 6.9: Velocity maps for water flows within the SHCD for a vertical, central slice, for seven different pumping rates, labelled by the corresponding inlet velocity respectively (left) for flow 'down' through the SHCD, (right) for flow in the reverse, or 'up' direction. The colour map images are rescaled as fractions of the observable field-of flow (FOF; the velocity space 'field-of-view' equivalent) for each to show the similarity of flow profiles in each instance. 

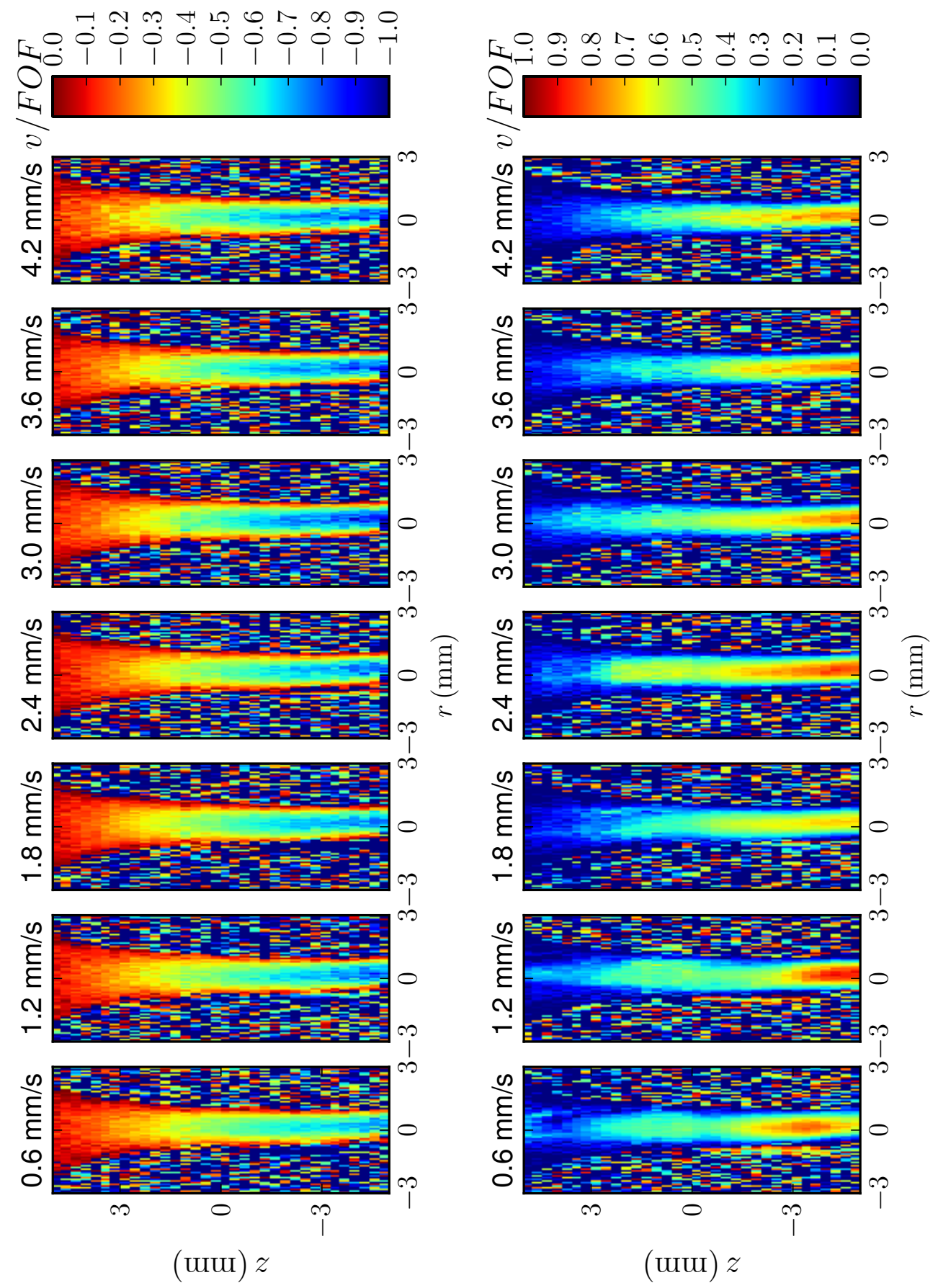

Figure 6.10: Velocity maps for Pluronic P105 solution flows within the SHCD for a vertical, central slice, for seven different pumping rates, labelled by the corresponding inlet velocity respectively (left) for flow 'down' through the SHCD, (right) for flow in the reverse, or 'up' direction. The colour map images are rescaled as fractions of the observable field-of flow (FOF; the velocity space 'field-of-view' equivalent) for each to show the similarity of flow profiles in each instance. 


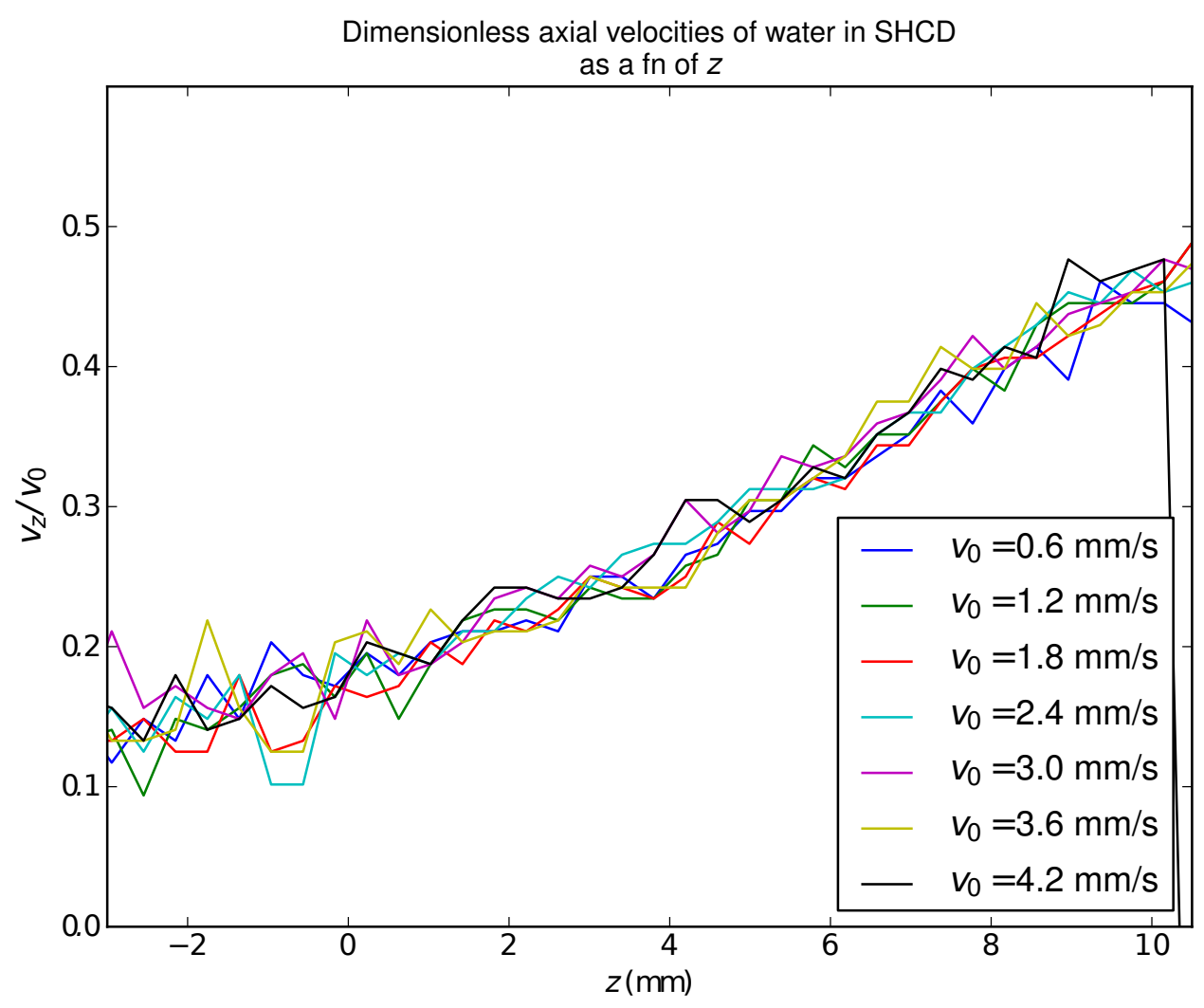

Figure 6.11: Velocities of a water sample along the central line of the SHCD are shown, as fractions of the field-of-flow. The acquired profiles are approximately linear and, given the equal spacing of the linear piston speeds and fields-of-flow chosen, are adequately collinear.

Clearly obvious is a difference in the level of fluctuation in the central (axial profile) velocity data between the water and P105 cases. It could be that the P105 sample shows more noisy data than the water due to a noise effect in the phase difference obtained for creating the propagator data, conceivable due to there only being two $q$-space data points being Fourier transformed, and hence a very broad propagator (peak picking errors may be easier to make). However the difference in raw signal-to-noise ratio between the water and P105 cases should be very small, and hence it may be that this variability may indicate some intrinsic flow fluctuations that are generated by extensional flow in this complex fluid.

The second difference in these two data sets is that the supposed linear 


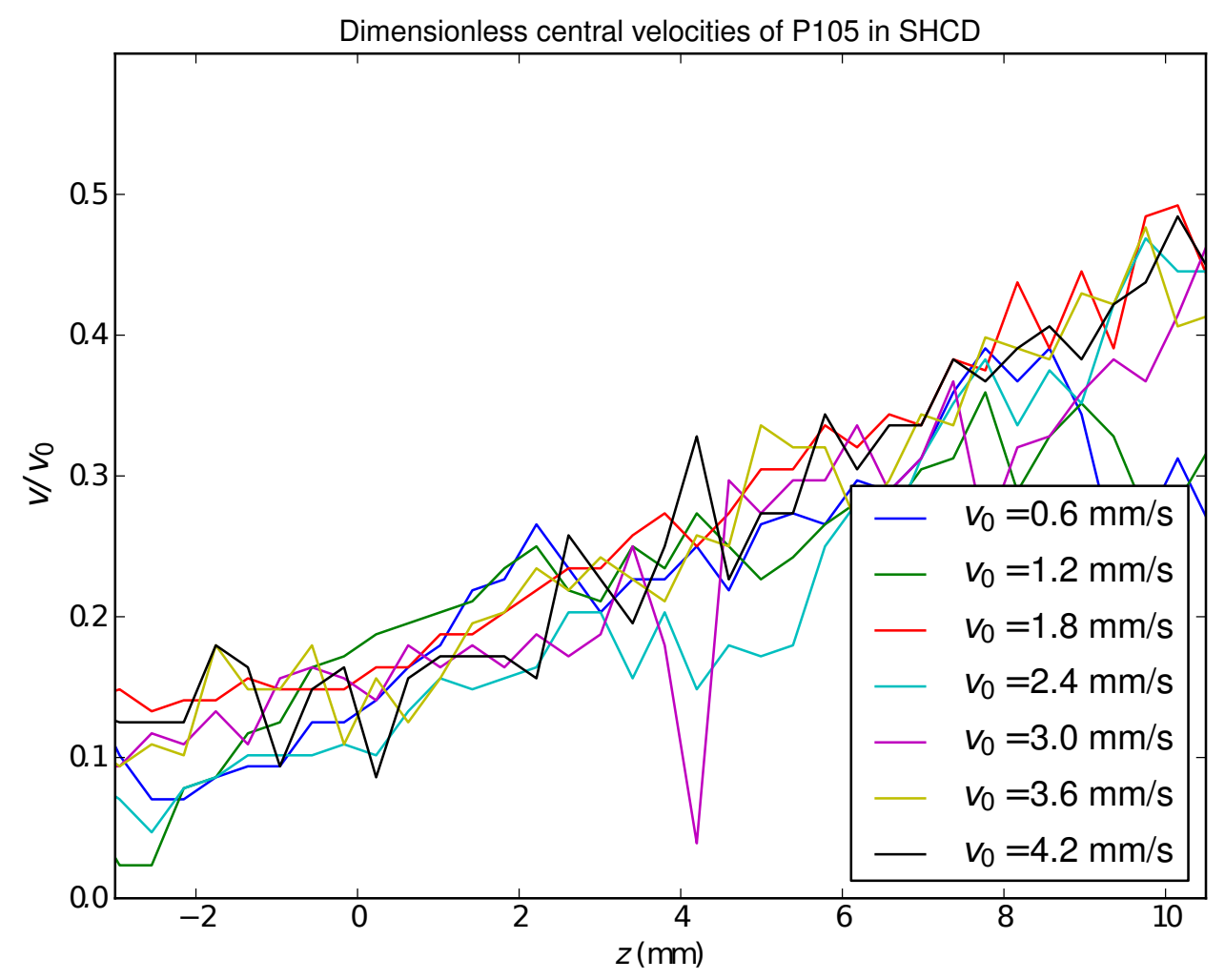

Figure 6.12: Velocities of our Pluronic P105 sample along the central line of the SHCD are shown, as fractions of the field-of-flow. The acquired profiles are approximately linear and, given the equal spacing of the linear piston speeds and fields-of-flow chosen, are adequately collinear.

increase in velocity along the length of the SHCD is not reflected as truly for the water as for the P105 central velocities. While the velocity profiles fall roughly along the same curve upon scaling, that curve is rather gently trending towards higher-than-expected velocities the further down the SHCD one moves. Of course, the SHCD is designed with the plug-flow scenario in mind, but in the case that the flow should be parabolic, we would still expect a linear increase in central velocities down the pipe, seeing as the maximum velocity in a circular parabolic flow is twice the average velocity of the flux over any plane. The only way that the central velocities would not reflect a linear increase is if the cross-pipe profiles take different functional forms as the flow progresses. The fact that the P105 central velocities show a more linear increase is interesting as 


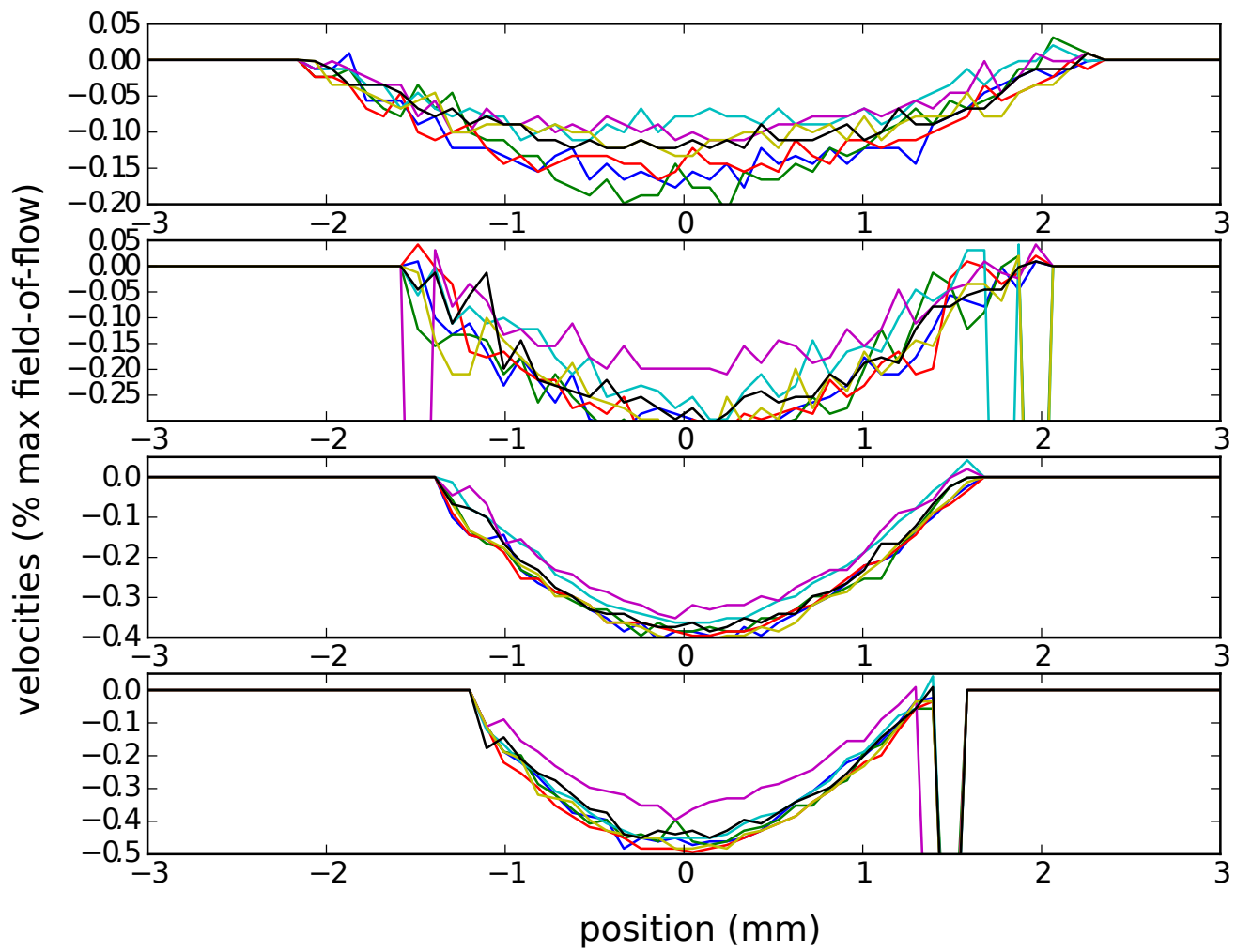

Figure 6.13: Axial velocities of water flow in transverse profile across the SHCD cell are shown. Each panel corresponds to one of four positions, situated at $0,3,6$, and $9 \mathrm{~mm}$ from the inlet. For each position, the seven inlet flow rates are shown, scaled to show similarity.

the shear-thinning properties dictate a more plug-like flow, and will be of note in further analysis.

Near-parabolic velocity profiles are seen across-the-pipe in the case of water flow, and this is consistent with our previous remarks regarding the non-plugflow-like behaviour attributable to a slightly non-linear increase in velocity with axial position. Also consistent with the P105 flow, the cross-pipe velocity profiles for the micellar solution flow are indeed flattened to some degree near the central point, indicating that the design condition (that the pipe be semi-hyperbolic) is more appropriate in this case. This reduction of the shear field in the central region also gives us confidence that spectroscopy might be able to carried out on "cylindrically symmetric" voxels, via the Hankel transformation technique. 


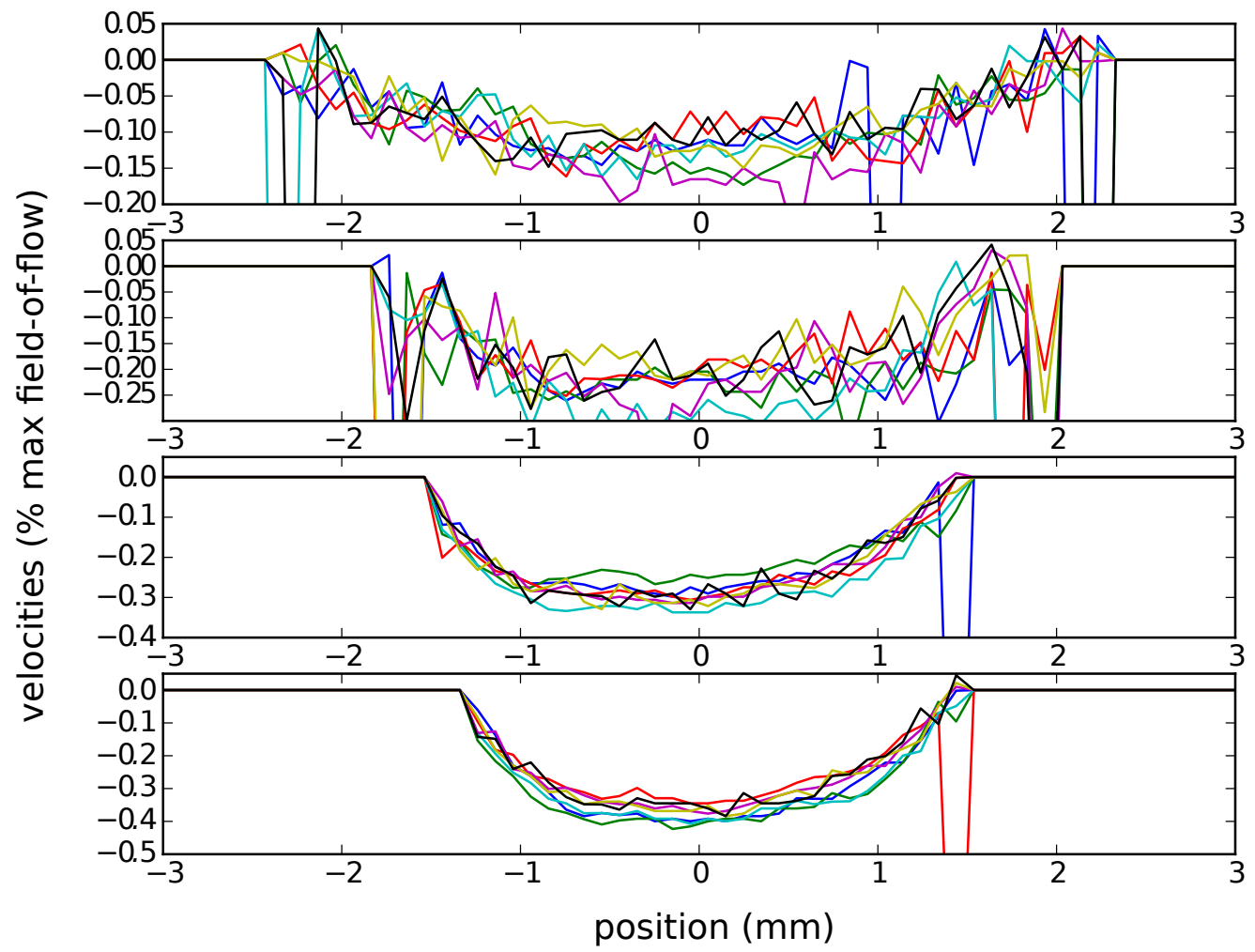

Figure 6.14: Axial velocities of our Pluronic P105 in transverse profile across the SHCD cell are shown. Each panel corresponds to one of four positions, situated at $0,3,6$, and $9 \mathrm{~mm}$ from the inlet. For each position, the seven inlet flow rates are shown, scaled to show similarity.

\subsubsection{Magnetic Resonance Spectroscopy in an SHCD}

Spectroscopic data is of vital importance in magnetic resonance investigations of rheological properties of materials, whether proton-based or using alternative nuclei such as deuterium. As ever, an experiment obtaining an image which retains spectroscopic information requires a dimensionality one greater than an density image alone. As a test-case, with water filling the SHCD, here we confirm the validity of the use of the Hankel transformation (see section 3.8.4) as being accurate qualitatively and consistent quantitatively for a scalar field derived from an axially symmetric pipe. With the goal of such spectroscopy being to use the shear-free core flowing through the SHCD as the interesting voxel for spectroscopic imaging, at first glance it may be unclear as to whether 
the reconstruction from Cartesian to a radial space is legitimate. At a second glance it seems a plausible procedure, and here some basic spectroscopic imaging experiments confirm this.
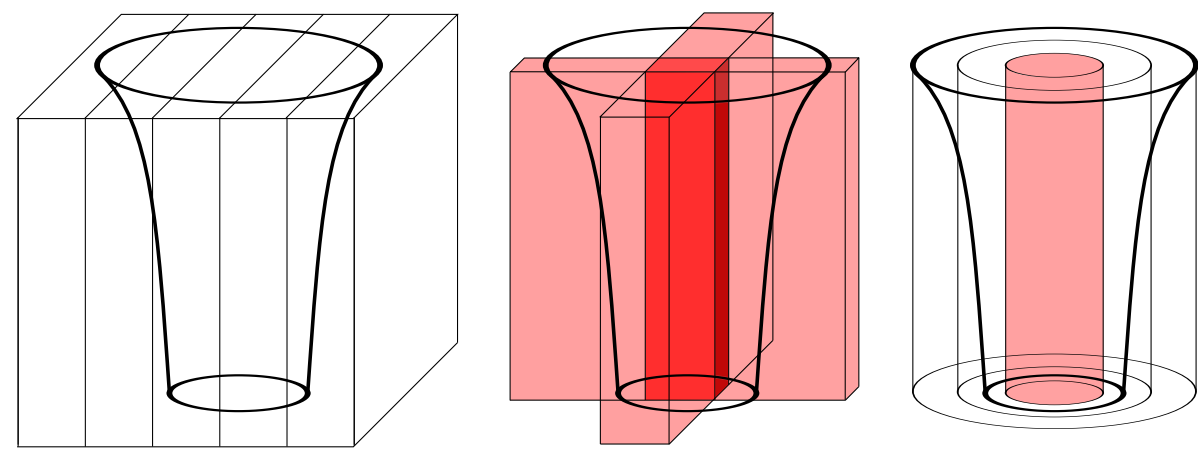

Figure 6.15: (left) Obtaining a phase-encoded one-dimensional image aggregates points into bins based on position as shown. Whether or not this is appropriately symmetric for the SHCD and Hankel transformation is to be shown, (centre) the alternative using two volume slice selections, generating a clumsy cuboid sensitive volume in the SHCD, and (right) the ideal voxels for the geometry and its intended use in extensional flow.

The benefits of being able to use such a technique are quite valuable. To selectively obtain spectroscopic data from the central shear-free core by conventional methods would require the imposition of at least two slice selective rf pulses (a third would be required if the $z$-direction were restricted as well). This in turn would mean that signal acquisition would occur at (a minimum of) two echoes from the excitation pulse. While this may not ordinarily restrict the type of experiments achievable, as we have seen in Chapter 5, the relaxation times for some micellar systems can be extremely short, and the most must be made of time in the pulse sequence. In any case, the circular effective voxel size in Hankel transformed data is much more suited to our geometry (though not quite as suited as in the case of the Couette geometry), and signal is obtained from an efficiently shaped volume. The only negative aspect to the use of the technique is that the signal-to-noise ratio for a particular voxel in radial space is proportional to the radius at which the voxel lies. This means that noise overwhelms the signal at the centre of the pipe, precisely the region of interest in our extensional flow geometry, however there may be enough signal near to, but not exactly on, the pipe axis. By judicious choice of voxel avergaging, however, it 
will be possible to offset some of these problems and obtain spectroscopic data adequately close to the centre of the volume of interest.

\subsubsection{Radial spectroscopic imaging of P105 in an SHCD}

We now turn to the task of obtaining spectroscopic information from the P105 sample, containing deuterated 1-phenylethanol: the task is to measure the NMR spectrum of deuterons present, which is an indicator of alignment of micelles in the flow. In a similar fashion to the spatially resolved spectroscopy obtained in chapter 5 a spin-echo, phase encoding pulse program was used to image in one direction only, though in contrast to that work a CSI (chemical shift imaging) routine built in Paravision, supplied by Bruker was used here. The spectra imaged across one spatial dimension were obtained for the $10 \mathrm{~mm}$-thick slice corresponding to the previously described semi-hyperbolic functional form of the contraction. The Hankel transformation was then used on this one-dimensional "imaging" result, in order to obtain the spectral data as a function of "radial voxel" (in the fashion of the right-hand schematic in fig. 6.15).

The results of this spatially resolved spectroscopic measurement can be seen in fig. 6.16, and several features are worth noting.

- Firstly, it is clear that as the flow speed is increased, the apparent intensity of the spectral features decreases, which maybe attributable to line broadening, which we will investigate with further analysis.

- Secondly, the four spectra shown at each flow rate are of differing intensities. As apparent from fig. 6.15, once the axis on which the Hankel transformation is chosen, locations corresponding to a particular co-ordinate on that axis are treated indiscrimantly. As such the 'outer' voxels may not contribute the same intensity signal as the 'inner' voxels, due to the contracting shape of the SHCD. This, however, does not intuitively explain the near-identical heights of the two 'inner' spectra - though upon further consideration it can be kept in mind that the Hankel transformation outputs signal as a function of radial position, which in our case is hopefully uniform once the voxel lies almost completely within the 'thin' end of the SHCD. 

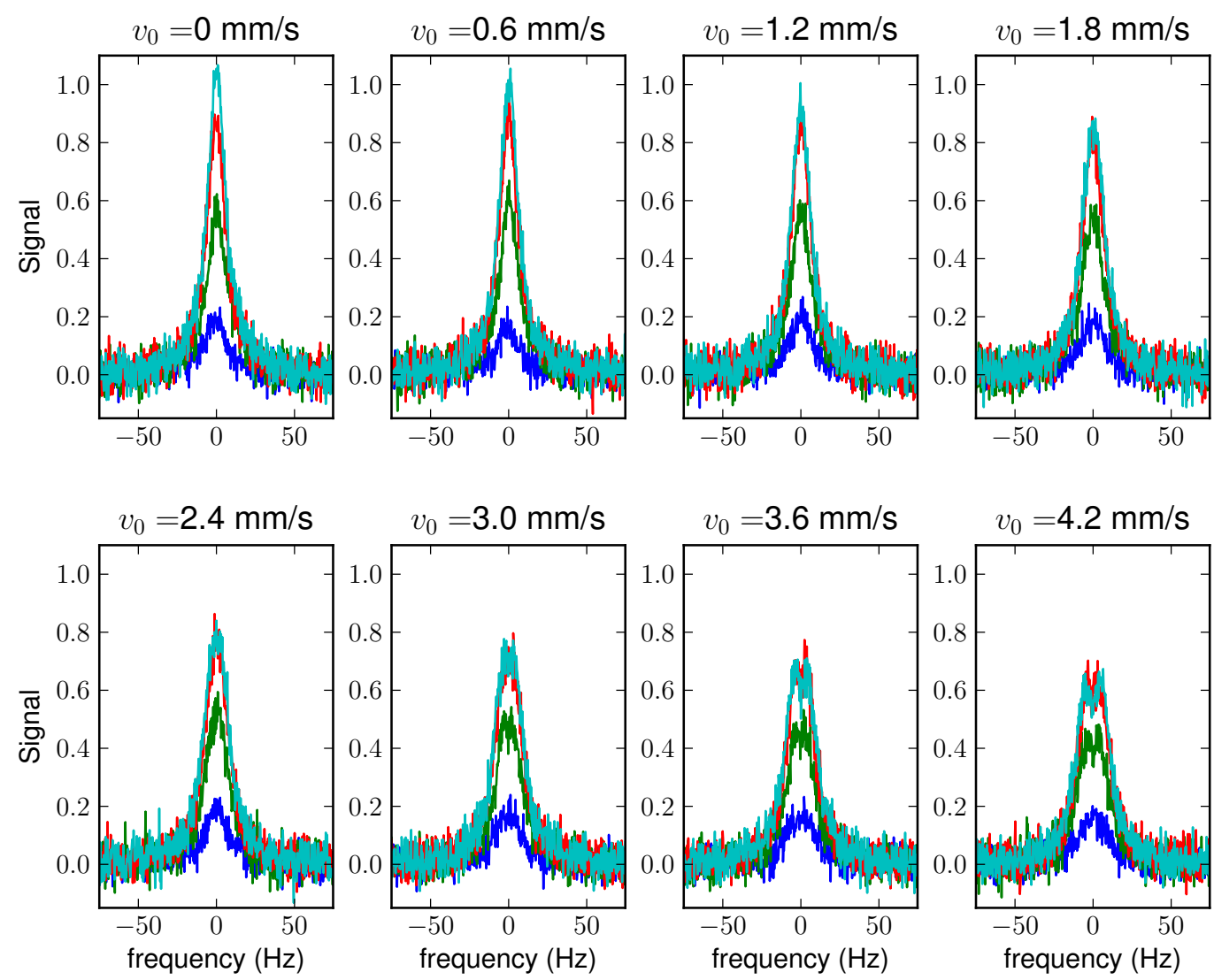

Figure 6.16: Here, the deuteron spectra of 1-phenylethanol in P105 are shown, as a function of inlet velocity. In each case, four "radial voxels" are obtained from the Hankel transformation, each centred $1 \mathrm{~mm}$ apart. The spectra correspond to position as follows: (Dark blue) The outer-most $(r=4 \mathrm{~mm})$ voxel; (Green) The second outer-most ( $r=3 \mathrm{~mm}$ ) voxel; (Red) The second inner-most $(r=2 \mathrm{~mm})$ voxel; and (Light Blue) the inner-most $(r=1 \mathrm{~mm})$ voxel.

- Thirdly, while a lowering of the peak with flow rate occurs, no easily apparent qualitative change occurs in the line shape with either position or flow rate, until the inlet flow rate reaches $6 \mathrm{~mm} / \mathrm{s}$. At that flow rate, some flattening or splitting of the top of the spectral line becomes seen, and is even more obvious at inlet speed of $7 \mathrm{~mm} / \mathrm{s}$.

\subsubsection{Further analysis of the spatially resolved spectra}

It is highly encouraging that at least some spectral features show some flow rate and position dependence, in particular given that that positional dependence is, 

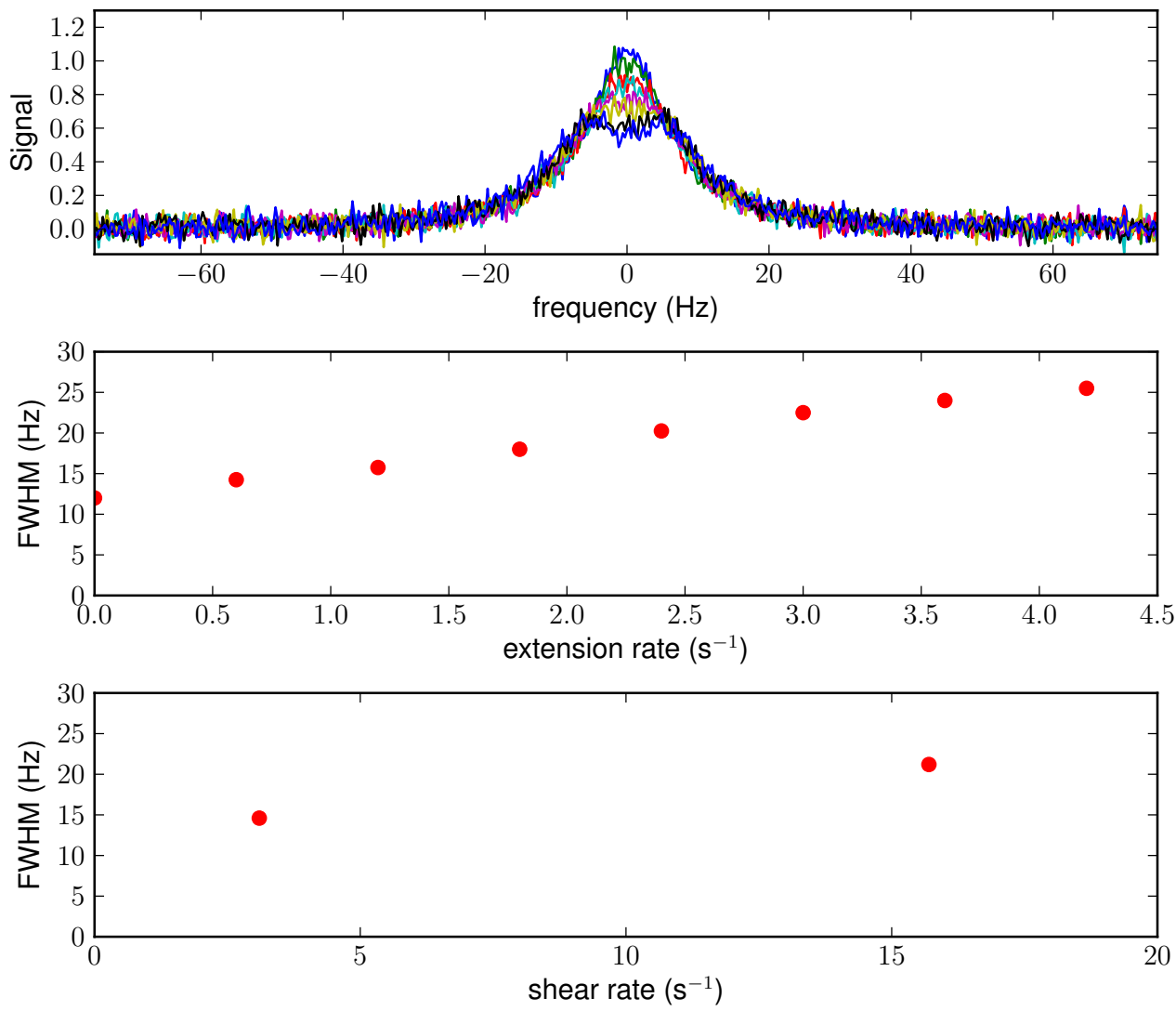

Figure 6.17: (Top) As a function of extension rate produced, the spectra corresponding to the "inner-most" voxel of the SHCD, the progression being from zero extension (highest peak) to highest extension rate (lowest, split peak), (middle) the measured full-width half-maxima of the spectra shown, as a function of the extension rate produced, and (bottom) by way of comparison at reasonably small deformation rates, the increase in linewidth for shear flow (see fig 5.3) of a P105 solution. 
by design, somehow related to the 'purity' of the extensional (relatively shearfree) properties of the flow. Figure 6.17 shows some information extracted from the data of figure 6.16, namely the linewidths. Analysis of these linewidths is performed by taking the absolute full-width half-maximum, whether the feature appears to be a single peak, or composed of two, split peaks.

We do not attempt to make definitive conclusions on this data, but make some comments based on their analysis later in the chapter. It is, however, interesting to note the evolution of the linewidth of the major spectral feature, in a direct comparison between this data and the spectral measurements seen in Chapter 5. In that chapter, changes in the deuteron NMR spectrum were observed, and while those experiments were highly temperature dependent, and not many low-valued shear rate data were obtained, reasonable initial comparisons lead to the observation of a variation in linewidth of approximately $\lesssim 0.6 \mathrm{~Hz} / \mathrm{s}^{-1}$ (see fig. 6.17(bottom), before the linewidth plateaued at higher strain (shear) rates. In contrast, the data of fig. 6.17(middle), being the extracted linewidths from deuterons passing through the SHCD, yield a strain (extension) rate dependence of linewidth increase of $3.8 \mathrm{~Hz} / \mathrm{s}^{-1}$. The final extension rate linewidth is very slightly less than a projected straight line continuation from lower extension rates, but whether this is a real sign that some plateau has commenced or not is not able to be stated.

\subsection{Conclusions}

We have explored the potential for magnetic resonance to be applied to the study of contraction flows in a semi-hyperbolic converging die (SHCD). In the first case, the design process for construction of an SHCD suitable for use in our Bruker micro-imaging magnet system was a step-wise process, the final implementation being a glass tube, shaped at one end by being drawn down onto a graphite former, which was lathed to provide a precisely defined functional form for the contraction flow. This functional form was determined by considering the throughput or flux through any axial plane across the tube, and with the choice of free parameters made, the radial profile for our glass SHCD was 


$$
R^{2}(z)=\frac{405}{16}\left(z+\frac{5}{4}\right)
$$

which results in an extension rate within the SHCD of $\dot{\varepsilon}=\frac{4}{5} v_{i}$, where $v_{i}$ is the inlet velocity.

Once flow-rate testing, leak prevention and simple characterisation of the extensional flow system with the conventional Rheo-NMR setup was complete, simple magnetic resonance imaging of the SHCD filled with water confirmed the quality of the interior tube shape.

Velocimetry was then carried out on a water sample, in the forms of figs 6.9 and 6.10, which, being scaled (normalised) colormaps, in most cases showed just how self-similar the flows were with changing flow-rate. From these maps, some of the most important intermediate data was obtained, namely the central axial velocity profiles. The SHCD's primary design feature is that the extension rate, or spatial rate-of-change of velocity, be linear. While the data obtained for the water characterisation showed some curvature in this aspect, this may possibly be rationalised by the consideration that the SHCD is designed based on the premise we are looking for shear-free flow fields, and that the velocity profile across-the-tube in some way becomes more plug-flow-like. Indeed, when replaced by the Pluronic solution P105, which has already featured in shear studies in Chapter 5, axial velocity profiles do indeed become more linear, however the velocity data does fluctuate more than in the water scenario. Hence we conclude that the designed form for the SHCD has been successful enough to be valuable in Rheo-NMR-based extensional flow studies of complex fluids.

A key indicator that Rheo-NMR can provide new information in such studies, however, is the use of techniques which can indicate molecular alignment. Extensional flow's transient nature, and the limited reservoir volume able to be used in our non-cyclic flow system, means that spectroscopic imaging (spatially resolved spectroscopy) is an ideal basis for carrying out interesting work in extensional Rheo-NMR on complex fluids. As such, we performed a spectroscopic imaging experiment on P105, by way of the deuterons within 1-phenylethanol stabilising molecules crucial for worm-like micelle formation, and the change in these spectra with varying shear rate and positions is shown in fig 6.16. Substantive conclusions are hard to make based on these plots, but it is incredibly 
heartening to see spectral variations, considering the small volumes involved, the residence time of relevant deuterons within that volume, and even the relative insensitivity of those deuterons. Quadrupolar interaction splitting of the spectrum was really only seen at the highest flow rates (and hence extension rates) used, which, upon reference to the literature, are not large extension rates by any stretch, however reliable data was successfully obtained in this research by compromise of fluid residence time and flow rate. The progression and dependence of deuteron spectral linewidth with increasing extensional deformation rate, and hence increasing spectral broadening and/or splitting, was measured to be $3.8 \mathrm{~Hz} / \mathrm{s}^{-1}$, approximately 6 times the similar dependence extracted from the shear deformation spectral data of chapter 5 . We do not attempt to attribute this to a certain extensional viscosity, however the fact that this ratio is greater than the Trouton ratio for Newtonian fluids, $\operatorname{Tr}=3$, is consistent with basic fluid mechanics theory. Hence, the conclusion we do make is that there is a possible future in Rheo-NMR studies for extensional flow, particularly further expanding the envelope of experimental capability. In our case we must provide faster techniques that allow higher flow rate experiments, so as to compare with other research on non-Newtonian fluids [23-27] to be able to make some sense of the large discrepancies seen in the literature $[1,10,28]$. 


\section{Bibliography}

[1] C.J.S. Petrie. One hundred years of extensional flow. Journal of NonNewtonian Fluid Mechanics, 137(1-3):1-14, 2006.

[2] C.J.S. Petrie. Extensional viscosity: a critical discussion. Journal of NonNewtonian Fluid Mechanics, 137(1-3):15-23, 2006.

[3] Fred. T. Trouton. On the coefficient of viscous traction and its relation to that of viscosity. Proceedings of the Royal Society of London. Series A, Containing Papers of a Mathematical and Physical Character, 77(519):426440, 1906.

[4] RG Larson. Instabilities in viscoelastic flows. Rheologica Acta, 31(3):213263, 1992.

[5] JRA Pearson. Instability in non-Newtonian flow. Annual Review of Fluid Mechanics, 8(1):163-181, 1976.

[6] JL White. Dynamics, heat transfer and rheological aspects of melt spinning: a critical review. Polym. Eng. Reviews, 1:297-362, 1981.

[7] DM Binding and K. Walters. On the use of flow through a contraction in estimating the extensional viscosity of mobile polymer solutions. Journal of Non-Newtonian Fluid Mechanics, 30(2-3):233-250, 1988.

[8] DM Binding. Further considerations of axisymmetric contraction flows. Journal of Non-Newtonian Fluid Mechanics, 41(1-2):27-42, 1991.

[9] S. Nigen and K. Walters. Viscoelastic contraction flows: comparison of axisymmetric and planar configurations. Journal of Non-Newtonian Fluid Mechanics, 102(2):343-359, 2002. 
[10] A. Keller and JA Odell. The extensibility of macromolecules in solution; a new focus for macromolecular science. Colloid $\& 3$ Polymer Science, 263(3):181-201, 1985.

[11] RI Tanner and RR Huilgol. On a classification scheme for flow fields. Rheologica Acta, 14(11):959-962, 1975.

[12] DV Boger, MJ Crochet, and RA Keiller. On viscoelastic flows through abrupt contractions. Journal of Non-Newtonian Fluid Mechanics, 44:267279, 1992.

[13] O. Hassager, J.M.R. Marin, K. Yu, and H.K. Rasmussen. Polymeric liquids in extension: fluid mechanics or rheometry? Rheologica acta, 49(6):543$554,2010$.

[14] R.G. Larson. The rheology of dilute solutions of flexible polymers: Progress and problems. Journal of rheology, 49:1, 2005.

[15] K. Feigl and HC Öttinger. The flow of a LDPE melt through an axisymmetric contraction: A numerical study and comparison to experimental results. Journal of rheology, 38:847, 1994.

[16] DF James, GM Chandler, and SJ Armour. A converging channel rheometer for the measurement of extensional viscosity. Journal of Non-Newtonian Fluid Mechanics, 35(2-3):421-443, 1990.

[17] D.F. James. Flow in a converging channel at moderate Reynolds numbers. AIChE journal, 37(1):59-64, 1991.

[18] DM Binding, PM Phillips, and TN Phillips. Contraction/expansion flows: The pressure drop and related issues. Journal of Non-Newtonian Fluid Mechanics, 137(1-3):31-38, 2006.

[19] K. Feigl, FX Tanner, BJ Edwards, and JR Collier. A numerical study of the measurement of elongational viscosity of polymeric fluids in a semihyperbolically converging die. Journal of Non-Newtonian Fluid Mechanics, 115(2-3):191-215, 2003. 
[20] DF James, GM Chandler, and SJ Armour. Measurement of the extensional viscosity of M1 in a converging channel rheometer. Journal of NonNewtonian Fluid Mechanics, 35(2-3):445-458, 1990.

[21] M. Shirakashi, H. Ito, and D.F. James. LVD measurement of the flow field in a constant-extensional-rate channel. Journal of Non-Newtonian Fluid Mechanics, 74(1-3):247-262, 1998.

[22] K. Feigl and H.C. Öttinger. A numerical study of the flow of a low-densitypolyethylene melt in a planar contraction and comparison to experiments. Journal of rheology, 40(1):21-36, 1996.

[23] J.P. Rothstein. Strong flows of viscoelastic wormlike micelle solutions. Rheology Reviews, The British Society of Rheology, Aberystwyth, Wales, UK, 2008.

[24] M. Cromer, L.P. Cook, and G.H. McKinley. Extensional flow of wormlike micellar solutions. Chemical Engineering Science, 64(22):4588-4596, 2009.

[25] D. Kivotides, S.L. Wilkin, and T.G. Theofanous. Entangled chain dynamics of polymer knots in extensional flow. Physical Review E, 80(4):41808, 2009.

[26] S.L. Ng, R.P. Mun, D.V. Boger, and D.F. James. Extensional viscosity measurements of dilute solutions of various polymers. Journal of NonNewtonian Fluid Mechanics, 65(2-3):291-298, 1996.

[27] GV Vinogradov, VD Fikhman, BV Radushkevich, et al. Viscoelastic and relaxation properties of a polystyrene melt in axial extension. Journal of Polymer Science Part A-2: Polymer Physics, 8(5):657-678, 1970.

[28] RG Larson. Spinnability and viscoelasticity. Journal of Non-Newtonian Fluid Mechanics, 12(3):303-315, 1983. 


\section{Chapter 7}

\section{Conclusions}

\subsection{Review of aims}

We set out in this thesis to explore ways in which magnetic resonance (MR) can play a contributing role to furthering knowledge of the rheological properties of complex fluids. Complex fluids are just so ubiquitous in everyday life that a lot of their properties are taken as simple, yet for many examples the truth dictates that we may not be so glib. Properties that interest us in this thesis are generally derived from the statistical properties of linear and pseudo-linear mesoscopic entities as they undergo motions within a matrix which is in itself statistical in nature. The bulk of ideas we investigate are founded on the idea of the tube model of polymers [1], the one-dimensional curvilinear diffusion of those polymers within the network [2], and that when such a material is deformed macroscopically and we treat a polymer molecule in isolation, we do not apply a force to the molecule, but rather just statistically affect the matrix in which it resides.

Flow constitutive equations relating the stress within a sample at some time to the history of the strain, are the primary job of the theoretical rheologist. The phenomenon of shear thinning has been worked on in theory and experiment alike for some time, however the related yet more conceptually opaque idea of shear banding does not have the same established foundation. Our major aim and underlying theme for this thesis was to investigate the way in which alignment of mesoscopic-level features of a polymeric material affects macroscopic 
rheological properties, and conversely how material deformation might affect microscopic structure. The method of expertise we bring to this investigation is magnetic resonance; the amalgamation of $\mathrm{MR}$ to rheology has been dubbed "Rheo-NMR" and, by inheritance from MR as a whole, it is a rich field. It incorporates MR spectroscopy, diffusometry, tomography, relaxation effects, and combinations of any of these in concert, not only in the case of proton magnetic resonance, but larger nuclei as well (particularly deuterons, in our case). As we have seen, deuterons have an intrinsic worth as a probe of alignment, due to their non-spherical nuclear charge distribution residing in the electric field gradient provided by electronic molecular orbitals. What follows is a summary of the way in which we have used deuterons to provide such information, though to begin with we review Chapter 4 - "Transverse Relaxation in Sheared Polymer Melts" as a way to explore the degree to which we may do away with deuteron spectroscopy altogether. In Chapter 5 - "Complex Fluids in Shear Flow", we mounted a campaign to tie alignment measurements with macroscopic properties, and found that deuterons are too valuable to totally ignore, especially in conjunction with the many other techniques at our disposal. In many senses, Chapter 6 - "Extensional Flow" is entirely complementary to the previous shear flow research, although there is far less extensive history to that field, though it is just as fundamental, rich and practical; we port many of the techniques employed in Chapter 5 to this chapter.

\subsection{Alignment's effect on proton spectroscopy of polymer melts}

The nucleus overwhelmingly recognised as being of most general utility, due to its abundance and high gyromagnetic ratio, is the smallest possible nucleus, the single proton of hydrogen. It does however lack some properties of the deuteron such as an electric quadrupole moment, and is therefore not used as much in molecular orientation-focussed research. Polymers, however, have several distinct timescales over which reorientation occurs at different lengthscales, and the through-space homonuclear internuclear dipole-dipole interaction that a particular nucleus feels will bear some signature of the director of the polymer 
tube segment in which it lies.

Following the work of Ball, Callaghan and Samulski (BCS), which was in turn based on the paper by Callaghan and Samulski, we set out to determine the form of the probability-based correlation function dictating the chance that a single proton nucleus would, after some time delay, be returned to the tube segment to which it belonged at the initial reference time. The two main contributions (and inhibitors) to the chances of being found as such are the one-dimensional curvilinear diffusion of the polymer "reptating" throughout the matrix of entanglements engendered by other polymers in the material, and the additional probability that such a tube segment still even exists at the time in question. These two probabilities, $\Psi_{R T O}$ and $\psi_{\text {tube }}$, respectively, totally determine a twopoint time correlation function

$$
C(t)=\langle\omega(t) \omega(0)\rangle
$$

where the value of omega is a function of the projection of the director of the tube segment along the Zeeman frame weighted by a sample-specific remanent dipole interaction strength. Initial numerical simulations indicated inaccuracies in the form of the magnetic resonance signal decay, and upon further reflection and an addition to the numerical code, we determined that, most importantly, a distribution of dipolar interaction strengths must be incorporated. This spread can be attributed to random fluctuations in local equilibration times and/or dissimilar diffusion rates locally within a macromolecule.

Comparison with the earlier experimental results of Dr. Ryan Cormier was the qualitative test of this numerical evaluation of the BCS theory of transverse relaxation. That experimental work was carried out on a $494 \mathrm{kDa}$ poly(dimethylsiloxane) melt, and the dependence of transverse relaxation time of the melt on the shear rate, and also the orientation of a horizontal Couette cell was measured. Correspondingly, our theory and simulation work contained in-built a model by which the direction of shear, in the hydrodynamic frame, could be re-oriented at will with respect to the magnetic resonance Zeeman frame - which could be viewed as allowing confirmation of the technique, or as an additional fitting parameter.

Three critical parameters of polymers in the melt phase were required to pro- 
duce the simulation relaxation data, those being the average number of polymer tube segments experienced by a molecule, $Z$, the average residual dipolar interaction strength, $\omega_{d 0}$, and the tube disengagement time, $\tau_{d}$. Preliminary values that were deduced by Cormier, and were hence used as initial guesses in our simulation were $Z=50$ and $\tau_{d}=210 \mathrm{~ms}$. No residual dipole interaction strength was proposed, however it was taken that this value should lie between 1 and $100 \mathrm{kHz}$.

Tuning these parameters and evaluating the quality of the fit to the experimental data, we obtained the following values

$$
\begin{aligned}
Z & =35 \pm 10 \\
\omega_{d 0} & =(3900 \pm 200) \mathrm{Hz} \\
\tau_{d} & =(600 \pm 300) \mathrm{ms}
\end{aligned}
$$

and conclude that, with an appropriate model of polymer dynamics, proton magnetic resonance can indeed be used to elucidate the degree of alignment, although the most drastic variation in transverse relaxation time is confined (for the polymer we investigated) to a shear rate range of $\sim 20 \mathrm{~s}^{-1}$.

\subsection{Rheo-NMR observation of the effect of shear on worm-like micelles}

An ideal test-bed for the investigation of the rheology of non-Newtonian fluids are worm-like micelles, being polymer-like in geometry, but in a looselyaggregated, self-assembled way. Cates and others have proposed constitutive equations relating the internal stress and strain history based on rates of breakage and recombination of such worm-like micelles, two mechanisms which allow for the presence of a variety of characteristic dynamical times not available to conventional polymers. The worm-like micelle used in our work was formed of the triblock copolymer (polyethylene oxide-polypropylene oxide-polyethylene oxide) material "Pluronic" P105, courtesy of BASF. Required to generate and maintain the worm-like structure, 1-phenylethanol is added to aqueous solu- 
tion of the Pluronic, and in its perdeuterated form also provides us with a mode of investigation of the alignment of the micelles, as the hydrophobic alcohol is presumed to reside in the core, provideed by the relatively hydrophobic polypropylene component of the triblock copolymer.

In the first place, it was established that this micellar solution was of considerable interest through conventional rheometry. In particular, the solution shows a narrow band of high viscosity in the temperature region between $\sim 290$ and $\sim 305 \mathrm{~K}$, but more than that, it shows an anomalous dip in viscosity at $\sim 295 \mathrm{~K}$ when sheared. Start-up stress measurements of the sample also indicated that at shear rates in the range 1.6 to $10 \mathrm{~s}^{-1}$, a very long (up to $10 \mathrm{~min}$ ) equilibration time may be required to come to steady state, confirming the unusual nature of this material.

Accordingly, a variety of key tools of Rheo-NMR were used to investigate the effect of shear on the Pluronic P105 solution. These included:

- ${ }^{1} \mathrm{H}$ spectroscopy and diffusometry, in order to determine the mobility of each component of the solution, and hence gain insight into the location of the phenylethanol, amongst other things,

- ${ }^{2} \mathrm{H}$ spectroscopy of the fluid, as a function of both temperature and shear rate. Such spectroscopy was immediately able to indicate that some internal structure of the fluid was indeed being modified within the temperature range indicated, as the quadrupole splitting which was readily apparent over that region was destroyed at specific temperatures within that region depending on the shear rate,

- spatially resolved velocimetry, which was used to show the velocity profile of the micelle material across-the-gap of the cylindrical Couette shear device. Two experiment types were carried out: one being a transient start-up view of the flow profile, which showed that, indeed, it took of the order of $10 \mathrm{~min}$ for the flow to come to a steady state, at which time the flow was clearly shear banded and; two, flow profiles across-the-gap showing how the steady-state evolved with increasing shear, a view of the material as one moves across the constitutive curve in a strain-rate controlled fashion. This traversal of the strain-rate axis showed different 
qualitative states, namely shear banding and fluctuations of the shearband interface, which were of the most interest, and

- spatially resolved deuteron spectroscopy. The use of the Hankel transformation procedure was of particular use here, due to the reasonably low abundance of deuterons and their inherently lower signal. Information from these experiments were intended to aid in the investigation of just whether a shear-band (a rheological concept) was correlated with a birefrigence band (as can be seen in a magnetic resonance experiment). In keeping with previous research, the correlation is not apparent, and many different combinations were observed: different alignment order parameters in a supposedly Newtonian regime appeared right alongside the case where no birefrigence banding occurred despite being abundantly shearbanded. This issue remains unresolved in terms of the experiments carried out here.

\subsection{Extensional flow as measured with mag- netic resonance}

Extensional flow has a chequered history, largely due to the transient nature of possible flow: one can only stretch something so far before some other material dimension must become prohibitively narrow or breakage occurs. Many different extensional rheometric devices have been designed and constructed, and in various classes of device and on many different samples, there is massive disagreement in quantitative results.

Magnetic resonance may be unable to clearly determine extensional viscosity, which would require a calibrated measurement of internal stress, but we feel it can still be of tremendous use in the field. The geometry chosen was the semi-hyperbolically converging die, so called for the functional form of a pipe constriction which supplies a flow field that, for plug-flow, dictates that the extension rate (the gradient of axial velocity) increases linearly along the pipe axis. This was eventually achieved using shaped glass, and successfully provided the correct shape, as evidenced by basic magnetic resonance imaging.

Following this, characterisation of the pipe (in terms of flow) was carried 
out on water. Magnetic resonance velocimetry was carried out, which showed near-linear increase of axial velocity with position along the pipe. What nonideality exists has been proposed to be an artifact of the non-globality of the pure extension field, in fact, an extended central volume absent of shear did not seem apparent for water at all.

In contrast, similar velocity mappings of the Pluronic solution containing phenylethanol, a worm-like micellar material, showed a much more linear progression along the pipe. Transverse profiles did not show quite the same "flat" region of plug-like flow within some radius from the axis, as had previosuly been seen before in the literature (where SHCD investigations were carried out with LASER Doppler Velocimetry). They were, though, certainly not qualitatively the same as the profiles of water, and it must be noted that the extension rates we explored up to are not nearly as large as those provided elsewhere, due to experimental constraints mentioned previously. Despite this, deuteron spectroscopic imaging was carried out, again with the use of the Hankel transformation, to determine whether the micelles in the Pluronic solution were being aligned along the flow direction. A steady change in spectral features was noted with both increasing extension rate, and as a function of radial position. Only four voxels were able to be pulled out of the data, however the forms of the spectra are not unreasonable, and in particular at the positions measured closest to the axis, at the highest extension rates, we can observe not only broadening of the deuteron peak, but some splitting as well.

Our research into the use of magnetic resonance in the assistance of rheological measurements of complex fluids bears the most potential of this thesis for future investigations. One area of note would be to improve the time resolution of the velocity imaging, so as to be able to push the extension rates observable by magnetic resonance higher. Another would be to design a pipe with not only long lead-in (giving the material a knowable strain history) but a long narrow end as well, so as to be able to investigate the reversibility properties of complex fluids. Our dominant conclusion for the final part of this thesis is that magnetic resonance can play an important part in the extensional rheology community, owing to its (recurringly) obvious noninvasive properties, able to probe flows without disruption. 


\section{Bibliography}

[1] M.Doi \& S.F.Edwards. The Theory of Polymer Dynamics. Oxford University Press, 1986.

[2] P-G. DeGennes. The Theory of Polymer Dynamics. Oxford University Press, 1971. 